Courrier hebdomadaire

$$
\mathrm{n}^{\circ} 2442-2443 \cdot 2019
$$

\title{
Le redéploiement économique de la Wallonie face à la diversité de ses territoires
}

\author{
Jean-Marie Halleux \\ Bruno Bianchet \\ Hubert Maldague \\ Jean-Marc Lambotte \\ Pierre-François Wilmotte
}

(RiSP 


\section{Courrier hebdomadaire}

Rédacteur en chef : Cédric Istasse

Assistante éditoriale : Fanny Giltaire

Le Courrier hebdomadaire est soutenu par l'Administration générale de l'Enseignement et de la Recherche scientifique de la Fédération Wallonie-Bruxelles. Il est également publié avec le concours du Fonds de la recherche scientifique-FNRS et de la Fondation universitaire de Belgique.

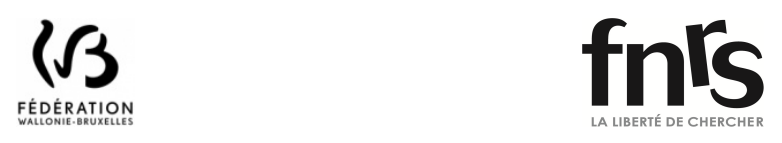

Une version numérique du Courrier hebdomadaire est disponible en pay per view (au numéro) et en accès gratuit pour les abonnés sur le site portail de CAIRN (http://www.cairn.info).

Le numéro simple : 6,90 euros - le numéro double : 12,40 euros

Abonnement : 235,00 euros

Souscription, commandes et informations :

CRISP - Place Quetelet, 1A - 1210 Bruxelles

Tél : 32 (0)2 2110180 - Fax : 32 (0)2 2197934

http://www.crisp.be - info@crisp.be

IBAN BE51 310027157662 - BIC BBRUBEBB

TVA 0408141158

Éditeur responsable : Jean Faniel - Place Quetelet, 1A - 1210 Bruxelles

Tous droits de traduction, d'adaptation ou de reproduction par tous procédés, y compris la photographie et le microfilm, réservés pour tous pays.

ISSN 00089664 


\section{TABLE DES MATIÈRES}

1. LIENS ENTRE TERRITOIRE ET DÉVELOPPEMENT ÉCONOMIQUE 10

$\begin{array}{ll}1.1 . \text { Quelques grilles de lecture } & 10\end{array}$

1.1.1. Mondialisation et économies d'agglomération 11

$\begin{array}{ll}\text { 1.1.2. La métropolisation } & 13\end{array}$

$\begin{array}{ll}\text { 1.1.3. Typologie des flux de revenus } & 16\end{array}$

$\begin{array}{ll}\text { 1.1.4. La périurbanisation } & 18\end{array}$

1.1.5. Attractivité, productivité et compétitivité 19

1.2. Les ressources territoriales 21

1.2.1. Le facteur connaissance et les systèmes régionaux d'innovation 21

1.2.2. La " classe créative » comme facteur d'attractivité des entreprises 23

1.2.3. Le capital humain et le déficit d'attractivité des RETI 24

1.2.4. Les aménités et la qualité des lieux 24

1.2.5. Les facteurs matériels directement utilisés par les entreprises :
les infrastructures de transport et le foncier à vocation économique

2. LES POLITIQUES PUBLIQUES EN MATIÈRE DE DÉVELOPPEMENT ÉCONOMIQUE ET TERRITORIAL $\quad 28$

2.1. Le rôle de l'Union européenne 28

2.1.1. Les orientations stratégiques de l'Union européenne 30

2.1.2. Les lignes directrices de la stratégie wallonne vis-à-vis de la programmation 2014-2020 de la politique régionale 31

2.1.3. Les choix infra-régionaux du gouvernement wallon et la géographie prioritaire des investissements 32

2.2. Les politiques économiques $\quad 36$

2.2.1. Le Plan Marshall 4.0 36

2.2.2. Les politiques de créativité et du numérique de l'autorité régionale wallonne 38

2.2.3. Les zones franches proposées par l'Autorité fédérale 39

3. LA WALLONIE DANS LE CONTEXTE DE L'EUROPE DU NORD-OUEST 41

3.1. Les environnements économiques de la Wallonie et de l'Europe du Nord-Ouest 42

3.1.1. L'argument de l'accessibilité privilégiée " au cœur de l'Europe » 42

3.1.2. Le PIB et le PIB par habitant 44

3.2. Les disparités socio-spatiales en Europe du Nord-Ouest à la lumière
des Objectifs de la stratégie Europe 2020

3.2.1. L'indice composite de suivi des objectifs du programme Europe $2020 \quad 47$

3.2.2. Analyse des composantes de l'indice composite 52

$\begin{array}{ll}\text { 3.2.3. En synthèse sur les provinces wallonnes } & 58\end{array}$

4. LA WALLONIE : UNE ÉCONOMIE PRODUCTIVE ET PRÉSENTIELLE 59

4.1. La Wallonie : une économie productive 60

4.1.1. Les grandes entreprises de la base productive 60

4.1.2. L'emploi de la base productive 62

4.1.3. L'emploi dans les secteurs éligibles selon le Programme opérationnel
FEDER 2014-2020

4.1.4. Les membres des pôles de compétitivité $\quad 65$ 
4.2. La Wallonie : une économie présentielle 68

4.2.1. Les revenus des résidents 68

4.2.2. Les emplois dans les secteurs du présentiel $\quad 71$

4.2.3. Le chômage 75

4.3. Typologie des communes wallonnes en fonction de leurs profils productifs et/ou présentiels $\quad 77$

5. LA STRUCTURATION DES TERRITOIRES WALLONS PAR LES AIRES MÉTROPOLITAINES TRANSFRONTALIÈRES 79

$\begin{array}{ll}\text { 5.1. Les flux de travailleurs transnationaux et transrégionaux } & 79\end{array}$

5.1.1. Quantification des flux de travailleurs transnationaux $\quad 80$

5.1.2. Quantification des flux de travailleurs transrégionaux 81

5.1.3. La géographie de la part des flux sortants $\quad 82$

5.2. Focus sur les aires métropolitaines $\quad 84$

5.2.1. L'aire métropolitaine de Bruxelles $\quad 84$

5.2.2. L'aire métropolitaine de Luxembourg 86

5.2.3. L'aire métropolitaine potentielle de l'Euregio Meuse-Rhin 86

5.2.4. L'aire métropolitaine potentielle de l'Eurométropole Lille-Courtrai-Tournai 88

6. LES RESSOURCES TERRITORIALES POUR LE DÉVELOPPEMENT FUTUR DE LA WALLONIE $\quad 89$

6.1. Le capital humain $\quad 90$

6.1.1. Le taux de diplômés de l'enseignement supérieur 90

6.1.2. La part de la population active appartenant à la classe créative 92

6.2. Les dynamiques entrepreneuriales et l'indice d'intensité de l'entrepreneuriat 95

6.3. Les secteurs porteurs pour le développement $\quad 97$

6.3.1. Les services supérieurs 97

$\begin{array}{lr}\text { 6.3.2. Les startups } & 100\end{array}$

7. PRINCIPAUX ENSEIGNEMENTS 103

7.1. Une trajectoire de redressement insuffisante 103

7.2. Économie productive versus économie résidentielle et présentielle $\quad 104$

7.3. Une Wallonie plurielle et sept grands ensembles territoriaux 106

7.4. Développement territorial et métropolisation 110

7.5. Vers une spécialisation des territoires : des politiques de développement à mieux adapter aux configurations territoriales

7.6. Des ressources territoriales à la géopolitique transfrontalière : un contexte ambivalent de concurrence et de coopération avec les régions voisines

7.7. Des questionnements en suspens et des besoins criants en données et en recherches 


\section{INTRODUCTION}

La question du déclin économique de la Wallonie est lancinante depuis des décennies ${ }^{1}$. Certes, le décrochage structurel subi lors de la seconde moitié du XX $\mathrm{XX}^{\mathrm{e}}$ siècle a cessé au tournant du XXI ${ }^{\mathrm{e}}$ mais, depuis lors, la convergence vis-à-vis des régions voisines, la Flandre en particulier, n'est toujours pas au rendez-vous. Le produit intérieur brut (PIB) par habitant et le revenu par habitant exprimés en pourcentage de la moyenne nationale en témoignent. Depuis 2000, ces deux indicateurs stagnent, aux alentours de $70 \%$ pour le premier et de $90 \%$ pour le second ${ }^{2}$.

Il est bien connu que les difficultés économiques qui affectent la Wallonie trouvent leur origine dans le déclin des secteurs qui en avait fait, avant la Ruhr, le premier bassin industriel moderne sur le continent européen. Il est également bien connu que les difficultés qu'éprouve la Wallonie pour redéployer son économie ne lui sont pas spécifiques ${ }^{3}$. Des problèmes similaires de maldéveloppement continuent d'affecter la plupart des régions européennes de tradition industrielle (RETI), dont le point commun est d'avoir connu une industrialisation précoce durant la première ou durant la deuxième révolution industrielle (cf. infra, 1.2.3).

Par rapport à la plupart des RETI, une singularité de la Wallonie réside dans son autonomie croissante. Suite aux réformes de l'État successives, la Région wallonne dispose de compétences très étendues, qui s'appliquent tant aux politiques de développement territorial (développement économique et aménagement du territoire) qu'aux politiques en matière de recherche et d'innovation technologique. Ces réformes institutionnelles, la sixième singulièrement, s'inscrivent dans une tendance vers la limitation des transferts financiers inter-régionaux. Par rapport aux RETI situées dans des États où la solidarité inter-régionale n'est pas en voie de minimisation, cette particularité pose avec davantage d'acuité la question de la contribution des agents économiques - les ménages et les entreprises - aux budgets des autorités en charge d'assurer les besoins collectifs.

* Réalisé par le Service de géographie économique (ECOGEO) et le Centre de recherche en sciences de la ville, du territoire et du milieu rural (Lepur) de l'Université de Liège (ULiège), ce travail s'est appuyé sur des matériaux issus de plusieurs recherches et, en particulier, de travaux financés par la Conférence permanente du développement territorial (CPDT) de la Région wallonne. Les auteurs tiennent donc à remercier cette structure tout en soulignant que le contenu publié reste de leur entière responsabilité.

M. QUÉVIT, Les causes du déclin wallon. L'influence du pouvoir politique et des groupes financiers sur le développement régional, Bruxelles, Éditions Vie ouvrière, 1978 ; J.-Y. HuWART, Le second déclin de la Wallonie. S'en sortir, Bruxelles, Racine, 2007.

2 C. AlBESSART et al., Rapport sur l'économie wallonne, Namur, IWEPS / DGO6 (SPW) / SOGEPA, 2017, p. 271. P. DestatTE (dir.), Innovation, savoir-faire, performance. Vers une histoire économique de la Wallonie, Charleroi, Institut Jules Destrée, 2005. 


\section{Un travail qui s'inscrit dans une perspective de géographie économique et qui porte sur la question du développement territorial}

Les travaux dédiés à la question du redéploiement de l'économie wallonne s'inscrivent le plus souvent dans la division de la Belgique en trois régions ${ }^{4}$. En préparant cette livraison du Courrier hebdomadaire, nous avons cherché à dépasser cette lecture en adoptant une perspective de géographie économique. Une telle perspective consiste à analyser la situation économique de la Wallonie, non pas à travers des moyennes globales et des tendances générales, mais à travers une analyse multi-échelle mettant en exergue l'enjeu de configurations sous-régionales très contrastées.

En plus de s'inscrire dans une perspective de géographie économique, la présente publication s'inscrit également dans une perspective de « développement territorial». Pour cerner ce que recoupe la notion le développement territorial, nous partirons de la définition générique suivante : le développement territorial correspond à la politique publique qui cherche à améliorer la performance des territoires vis-à-vis d'ambitions collectives de développement.

La politique du développement territorial réunit notamment les mondes du développement économique et les mondes de l'aménagement et de l'urbanisme. Idéalement, une telle politique est à mettre en ouvre via la coordination d'acteurs qui interviennent à différents niveaux de pouvoir et pour des politiques sectorielles variées. Dans cette perspective, la notion de développement territorial est proche des notions de gouvernance. Idéalement, une politique efficace de développement territorial est également à appliquer sur la base d'un projet de développement établi par concertation et grâce à la maîtrise de l'information territoriale. C'est ici la proximité entre les notions de développement territorial et d'intelligence territoriale qui est à souligner.

\section{Les disparités socio-spatiales en termes de développement et de ressources territoriales}

Les niveaux de développement des territoires qui composent la Wallonie sont très inégaux. Par exemple, il y a peu de points communs entre, d'un côté, le Brabant wallon, qui a peu de choses à envier aux régions européennes les plus prospères et les plus dynamiques, et, d'un autre côté, l'atonie qui paupérise des parties substantielles du territoire régional. Un des principaux objectifs de la présente publication est de mettre en exergue ces niveaux de développement différenciés ou, en d'autres termes, ces disparités socio-spatiales.

Afin de rendre compte des disparités socio-spatiales en termes de niveau de développement, nous allons nous intéresser aux disparités socio-spatiales en termes de ressources territoriales. Par ressources territoriales, nous entendons les caractéristiques des territoires qui permettent d'y attirer des flux de revenus.

Les flux de revenus qui irriguent les territoires dépendent de l'économie productive, via la capacité des firmes à générer de la valeur ajoutée et à exporter des biens et des services,

\footnotetext{
Notons l'exception suivante : Y. DE WASSEIGE, «Le développement des arrondissements wallons. Essai d'analyse comparée », Courrier hebdomadaire, CRISP, n 1624, 1998.
} 
mais aussi de l'économie résidentielle et présentielle ${ }^{5}$. La notion d'économie résidentielle renvoie à l'impact sur l'économie locale de revenus de résidents générés ailleurs, grâce à des migrations pendulaires de travail ${ }^{6}$, grâce à des pensions de retraite ou grâce à des prestations sociales. La notion d'économie présentielle est plus large que celle d'économie résidentielle. Elle intègre l'impact des revenus de résidents générés ailleurs mais également l'impact des personnes qui dépensent sur un territoire sans y être domiciliées. Il s'agit des touristes et des excursionnistes, mais également des navetteurs et des étudiants non résidents. Au final, la différenciation entre économie productive et économie présentielle vise à intégrer la disjonction spatiale croissante entre les lieux de production où les revenus sont générés et les lieux de consommation où les revenus sont dépensés ${ }^{7}$.

\section{L'enjeu des revenus qui irriguent les territoires}

En raison de la perspective de géographie économique adoptée, notre apport en termes de développement territorial se positionne prioritairement au niveau de la sphère économique. Pour être plus précis, en nous focalisant sur la thématique des ressources territoriales, nous plaçons la question des revenus au centre de nos préoccupations et nous partons du postulat que le développement des territoires - en particulier celui de la Wallonie - nécessite une hausse des revenus qui les irriguent. Bien que remis en cause par les paradigmes de la décroissance ou de la prospérité sans croissance ${ }^{8}$, ce postulat demeure solidement ancré auprès des décideurs et experts wallons, comme en témoigne le passage suivant tiré du préambule du rapport scientifique du Plan Horizon $2022^{9}$ : « Les travaux des experts "valident" clairement cette démarche gouvernementale. En effet, plusieurs d'entre eux insistent sur l'urgence d'une approche globale pour s'attaquer aux faiblesses de l'économie wallonne et faire face aux différents défis environnementaux et sociaux auxquels la Wallonie est et sera confrontée dans les prochaines années. En effet, si celle-ci a réussi à arrêter son "décrochage" par rapport à la Flandre, il n’en reste pas moins que son PIB par habitant reste très insuffisant par rapport à la moyenne belge et se doit de progresser tout en intégrant les contraintes liées la préservation de notre environnement et au caractère limité de certaines ressources ».

Le Plan Horizon 2022 a été préparé à l'initiative du gouvernement wallon, dans la perspective des modifications de la loi spéciale de financement et des transferts de compétence opérés par la sixième réforme de l'État. Comme l'illustre le passage cité, la philosophie du Plan Horizon 2022, à l'image d'ailleurs des autres plans wallons, en particulier les Plans Marshall successifs, repose explicitement sur l'idée que la croissance

5 L. DAVEZIES, «L'économie locale "résidentielle" ", Géographie, économie, société, volume 11, n 1, 2009, p. 47-53 ; M.-L. DE KEERSMAECKER et al., "Économie résidentielle et compétitivité des territoires », Territoires(s) wallon(s), hors série, 2007, p. 21-34.

6 Les migrations pendulaires de travail, aussi appelées navettes de travail, correspondent aux déplacements domicile-travail réalisés sur une base quotidienne ou quasi quotidienne.

D. GueX, O. CrevoISIER, «Globalisation postindustrielle et milieux locaux : une typologie », Working Paper MAPS, $\mathrm{n}^{\circ}$ 1, 2017 ; R. CAMAGNI, «Attractivité et compétitivité, un binôme à repenser », Territoires 2030, $\mathrm{n}^{\circ} 1,2005$, p. 11-15.

8 T. JACKSON, Prospérité sans croissance. La transition vers une économie durable, Bruxelles / Namur, De Boeck / Etopia, 2010.

S. BRUNET et al. (dir.), Horizon 2022 : rapport scientifique (version finale), Namur, Gouvernement wallon, 2013, p. 9. 
du PIB est un préalable pour rencontrer les défis environnementaux et sociaux auxquels la Wallonie est confrontée.

Le fait de postuler que le développement des territoires continue de nécessiter une croissance économique ne signifie pas pour autant qu'il faille considérer que la politique du développement territorial se limite à des aspects économiques. De même, il ne faut pas assimiler le PIB à un indice de bien-être ${ }^{10}$. En réalité, une approche complète du développement territorial se doit d'également intégrer les dimensions de la soutenabilité environnementale et de la cohésion sociale. Bien qu'il s'agisse là d'enjeux fondamentaux pour le bien-être de long terme des populations, ces thématiques s'écartent du focus que nous avons privilégié dans ce Courrier hebdomadaire. Nous ne les aborderons donc que de manière indirecte.

\section{Mondialisation et métropolisation}

L'enjeu des revenus qui irriguent les territoires est indissociable des évolutions vers l'éclatement géographique des processus productifs et vers les continentalisation et globalisation de l'économie productive. La concurrence globalisée pousse les firmes à placer les territoires en compétition pour des tâches productives de plus en plus segmentées (la conception, le management, le marketing, les différentes phases de production matérielle, la logistique, etc.). Cette concurrence globalisée appelle à des articulations fortes avec les territoires. En effet, elle pousse les firmes à implanter leurs diverses tâches dans des localisations où elles peuvent les assurer avec le plus d'efficience, renforçant de la sorte les disparités socio-spatiales et les écarts entre les régions qui "gagnent » et les régions qui « perdent » ${ }^{11}$.

Le concept de "métropolisation » est également au cœur de notre problématique. Il peut être défini comme le renforcement du poids et du rôle des grandes villes - les métropoles dans l'économie. Nous verrons que les ressorts de la métropolisation sont pluriels. La croissance des métropoles tient tant à leur interconnexion dans les réseaux continentaux ou mondiaux qu'à leur capacité à soutenir les firmes dans leur quête d'innovations.

La géographie économique de la Wallonie pose question vis-à-vis de la métropolisation. En son sein, nous n'y trouvons pas de métropole complète qui serait à la fois un foyer d'innovation majeur et une place centrale pour les services de commandement ${ }^{12}$. Par contre, la Wallonie subit fortement l'impact de pôles métropolitains extérieurs. Il en résulte un contexte ambivalent de concurrence et de coopération avec les régions voisines. Il en résulte également des dynamiques transfrontalières, transrégionales comme transnationales, qui entraînent un fort impact sur les disparités socio-spatiales. Pour le développement territorial de la Wallonie, les dynamiques transfrontalières peuvent être positives, à l'image des flux de frontaliers sortants qui induisent des flux de salaires entrants.

10 C. RUYTERS et al., Indicateurs complémentaires au PIB : l'indice des conditions de bien-être en Wallonie ( $2^{e}$ exercice), Namur, IWEPS, 2015.

1 Cf., entre autres, G. BENKO, A. LIPIETZ, Les régions qui gagnent, Paris, Presses universitaires de France, 1992 ; O. CREVOISIER, J. CORPATEAUX, A. THIERSTEIN, Intégration monétaire et régions : des gagnants et des perdants, Paris, L'Harmattan, 2001.

12 C. Vandermotten, J.-M. Decroly, « Géographie», in F. Joris (dir.), Wallonie. Atouts et références d'une Région, Namur, Région wallonne, 1995, p. 85-108. 
Mais elles peuvent aussi être négatives, à l'image des concurrences qu'exercent les territoires voisins pour l'accueil d'activités économiques.

\section{Les principaux objectifs et la structure de la publication}

Face aux enjeux du redéploiement économique de la Wallonie, l'objectif du présent travail est d'apporter un éclairage contemporain sur la manière dont les territoires wallons - au pluriel vu leur diversité - sont susceptibles d'y contribuer. Afin d'apporter cet éclairage, nous allons étudier la géographie économique du territoire wallon en analysant les disparités socio-spatiales de celui-ci. Un autre objectif de notre travail est de questionner l'efficacité des politiques publiques en matière de développement économique et territorial. Tout au long du travail, nous ne manquerons donc pas de proposer quelques suggestions visant à alimenter la réflexion sur les politiques publiques.

C'est afin de répondre à ces objectifs que nous avons structuré la publication en sept chapitres. Le premier chapitre synthétise un état de l'art sur les liens entre territoire et développement économique. Son objectif principal est de fournir des grilles de lecture pour les chapitres ultérieurs consacrés aux grandes dynamiques économiques et territoriales qui marquent la Wallonie. Le deuxième chapitre décrit les principales politiques publiques qui ressortent du champ du développement territorial et qui ont un impact sur le développement économique de la Wallonie. Ce chapitre est l'occasion de présenter les politiques régionales européennes ainsi que les politiques menées en Wallonie dans le champ du développement régional. À l'image du premier chapitre, ce deuxième chapitre vise prioritairement à préparer le lecteur aux analyses des dynamiques économiques et territoriales des chapitres suivants.

$\mathrm{Au}$ troisième chapitre, nous débutons la présentation de nos travaux empiriques par des analyses menées au niveau de l'Europe du Nord-Ouest. C'est à cette échelle que nous évaluerons comment la Wallonie et ses provinces se positionnent vis-à-vis des grands objectifs de compétitivité formulés par la stratégie Europe 2020 de l'Union européenne. Au quatrième chapitre, l'analyse est affinée par des traitements spécifiques sur la Wallonie et ses territoires. Ce chapitre est l'occasion de présenter les principales caractéristiques de la Wallonie en termes d'économie productive et d'économie résidentielle. Avec le cinquième chapitre, nous affinons la question des disparités socio-spatiales avec un focus sur les aires métropolitaines transfrontalières. Au sixième chapitre, c'est la question des ressources territoriales qui est passée en revue, afin de mettre en exergue les atouts sur lesquels la Wallonie pourrait s'appuyer pour son développement futur.

La publication se termine par un chapitre conclusif, qui présente les principaux enseignements de notre travail. Nous y synthétisons une lecture de la situation wallonne en termes de disparités socio-spatiales et nous nous appuyons sur cette synthèse pour formuler quelques pistes de réflexion susceptibles de renforcer l'efficacité des politiques publiques. 


\section{LIENS ENTRE TERRITOIRE ET DÉVELOPPEMENT ÉCONOMIQUE}

Ce chapitre constitue un substrat théorique utile à l'appréhension de la question des liens entre territoire et développement économique. Nous y présentons d'abord diverses grilles d'analyse sur ce sujet des liens entre territoire et économie. Ensuite, nous y développons la thématique des ressources territoriales.

\subsection{QUELQUES GRILLES DE LECTURE}

Les grilles de lecture présentées portent tant sur l'économie productive que sur l'économie résidentielle et présentielle. Rappelons que l'économie productive est à considérer dans un contexte de continentalisation et de mondialisation de la vie économique. Pour un territoire, les flux de revenus qui y correspondent dépendent de la capacité de ses firmes à exporter des biens et des services.

Dans les territoires, l'économie productive est complétée par l'économie présentielle. Localement, l'économie présentielle est d'autant plus vitale que le bien-être des habitants est basé sur d'autres types de ressources que les revenus des firmes exportatrices. Pour compléter ces revenus d'exportation, il faut attirer sur place divers profils de populations susceptibles de dépenser des revenus générés ailleurs. Parmi ces populations, nous trouvons des résidents permanents (principalement des navetteurs et des retraités), mais aussi des personnes qui sont présentes sur le territoire sans qu'elles n'y aient implanté leur résidence principale (des excursionnistes, des touristes, des étudiants, etc.). Cette distinction explique pourquoi, comme nous l'avons déjà relevé dans l'introduction générale, la notion d'économie présentielle englobe celle d'économie résidentielle ; en effet, elle intègre tant l'impact des résidents permanents que des personnes qui ne sont que de passage.

Les deux premières grilles de lecture développées dans ce chapitre sont celles des économies d'agglomération et de la métropolisation. Les approfondir clarifiera le sujet de l'économie productive. La troisième grille de lecture sera celle d'une typologie des flux de revenus. Présenter cette typologie sera l'occasion de préciser le sujet de l'économie résidentielle et présentielle. La quatrième grille de lecture est celle de la périurbanisation, un mouvement structurel qui ne doit pas être confondu avec la métropolisation, bien qu'il s'agisse là de deux phénomènes à forte dimension géographique. La cinquième grille de lecture vise à définir et à articuler les trois notions d'attractivité, de productivité et de compétitivité. 


\subsubsection{Mondialisation et économies d'agglomération}

Le concept d'économie d'agglomération est devenu central en géographie économique et en économie régionale. Il vise en particulier à appréhender la situation apparemment paradoxale des économies contemporaines où la mondialisation de l'économie s'accompagne à la fois d'un effacement des distances et d'une concentration - d'une agglomération - de l'activité productive au sein des aires urbaines les plus importantes.

Par effacement des distances, nous entendons les progrès intervenus dans les systèmes de transports. Ces progrès ont concerné la vitesse et la capacité des véhicules, du navire à l'avion en passant par le train, cela en relation étroite avec leur spécialisation de plus en plus poussée. En parallèle, nous avons assisté au développement de la conteneurisation, ce qui a facilité les transports multimodaux et engendré un développement extraordinaire des échanges internationaux par voie maritime. Cette véritable « révolution des transports » a entraîné non seulement le raccourcissement relatif de l'espace-temps, mais aussi la massification des flux de personnes et de biens. En outre, nous vivons aujourd'hui à l'ère du numérique. Grâce au développement des technologies de l'information et de la communication (TIC), des dispositifs de plus en plus variés permettent l'échange d'informations sans la coprésence. En conséquence, certains théoriciens ont prophétisé la fin de la distance, et donc la fin de la géographie, en laissant supposer que les facteurs de localisation ayant fait des villes les principaux foyers de l'innovation et les principaux noyaux de la vie économique devenaient obsolètes. Sur le terrain, c'est la tendance inverse qui s'est renforcée. En effet, partout sur la planète, la production de richesse continue à se concentrer au sein des régions urbaines les plus avancées ${ }^{13}$.

La concentration et l'agglomération des activités dans l'espace engendrent à la fois des externalités positives et des externalités négatives ${ }^{14}$. Intuitivement, les externalités négatives induites par la concentration sont les plus faciles à saisir. Il s'agit de la pollution, de la congestion des circulations et de la hausse du coût des facteurs de production (tant le foncier que la main-d'œuvre). En parallèle, l'agglomération des activités économiques au sein de territoires peu étendus se traduit par des externalités positives, dénommées économies d'agglomération par la littérature scientifique. Cette littérature distingue trois grandes catégories d'économies d'agglomération: les externalités d'informations, les externalités liées aux marchés du travail et les externalités liées à la présence de nombreux fournisseurs et clients.

Aujourd'hui, la compétitivité des firmes dans le contexte de l'économie de la connaissance exige des compétences toujours plus aiguës afin d'anticiper les évolutions des systèmes productifs. Disposer des informations stratégiques est donc une nécessité vitale pour la capacité d'innovation des entreprises, comme le résume parfaitement Pierre Veltz en s'appuyant sur les notions de réseau territorial et de milieu : «Un réseau territorial et/ou un réseau professionnel actif agissent comme des filtres qui permettent d'isoler les données les plus pertinentes et de sélectionner les actions les plus efficaces. Ceci concerne en

13 Banque mondiale, Repenser la géographie économique. Rapport sur le développement dans le monde, Bruxelles, De Boeck, 2009.

14 La notion d'externalité renvoie à l'impact de l'activité d'un agent économique (un producteur ou un consommateur) sur un autre agent économique sans que l'impact soit accompagné d'une contrepartie financière, c'est-à-dire sans rétribution pour les externalités positives et sans indemnisation pour les externalités négatives. 
particulier les jugements concernant les décisions les plus difficiles, dont la rationalité économique est sur le fil du rasoir. Faut-il acquérir telle machine automatisée, faut-il aller sur tel marché, faut-il faire confiance à tel centre de recherche, ou à tel fournisseur ? Face à des décisions subtiles de ce type, l'entrepreneur isolé souffre d'un grave handicap par rapport à celui qui appartient à un milieu - c'est-à-dire à une collectivité où circulent des connaissances pratiques inaccessibles à ceux qui n'en font pas partie » ${ }^{15}$.

Les externalités d'informations peuvent être renforcées par des politiques publiques de clustering, qui visent à stimuler l'innovation en favorisant les contacts entre entreprises ainsi qu'entre entreprises et centres de recherche ${ }^{16}$. Pour ne parler que de la Wallonie, c'est avec cet objectif que les outils du cluster, et ensuite du pôle de compétitivité, ont été institutionnalisés ${ }^{17}$. Pour autant, il est indéniable que la proximité induite par la concentration urbaine continue de représenter un puissant facilitateur de mise en contact. En outre, la proximité physique tend à renforcer les effets des dispositifs institutionnels mis en place par les politiques de clustering ${ }^{18}$.

Aux yeux des entreprises, les grandes régions urbaines - que nous appellerons les régions métropolitaines - présentent des avantages tant pour l'accès à l'information que pour l'accès à un vaste marché du travail partagé. Pour les entreprises, la probabilité de trouver facilement une main-d'œuvre adéquate augmente avec la taille des bassins d'emplois. Cela s'explique d'abord par de simples raisons quantitatives liées à la taille démographique des bassins. Cela s'explique ensuite par des raisons liées à la qualité de la main-d'œuvre ; en effet, les niveaux de qualification tendent à augmenter avec la taille des villes. Enfin, les coûts du désengagement sont socialement et économiquement plus faibles dans les zones métropolitaines; « cet argument implicite pèse lourd pour des groupes industriels qui sont presque tous impliqués dans des opérations douloureuses de désengagement dans des villes petites ou moyennes, où leur responsabilité d'employeur est cruellement exposée ${ }^{19}$.

Au-delà des externalités d'informations et de partage des marchés du travail, les externalités liées à la présence de nombreux fournisseurs et clients sont également un des facteurs explicatifs du poids croissant des grandes villes dans l'économie productive. Un avantage fondamental des pôles métropolitains tient ici à la forte concentration des services supérieurs aux entreprises (comptabilité, immobilier, marketing, informatique, etc.). Alors que les firmes tendent de plus en plus à se focaliser sur leur cour de métier, l'accès aisé à des services spécialisés est devenu un vecteur fondamental de leur compétitivité ${ }^{20}$. Considérons l'exemple d'une société de services informatiques qui connaît parfaitement les outils de ses clients, outils qui doivent être révisés et mis à jour au pied levé. $\mathrm{Vu}$ leur importance, il faut donc considérer les services supérieurs aux entreprises comme

15 P. Veltz, Mondialisation, villes et territoires. L'économie d'archipel, $2^{\mathrm{e}}$ édition, Paris, Presses universitaires de France, 2005, p. 230

6 M. PORTER, "Clusters and the new economy of competition ", Harvard Business Review, volume 76, nº 6, 1998, p. 77-90.

1 V. LEPAGE, « Le clustering en Wallonie », Territoire(s) wallon(s), hors-série $n^{\circ}$ 1, 2007, p. 119-126.

18 P.-F. WilmotTe, J.-M. HAlleuX, « La structure spatiale des systèmes régionaux d'innovation : qu'en est-il de la proximité géographique au sein des pôles de compétitivité ? ", L'Espace géographique, volume 47, $\mathrm{n}^{\circ} 1,2018$.

19 P. Veltz, Mondialisation, villes et territoires, op. cit., p. 255.

20 M. Bourgeois, J.-M. Halleux, G. Pagano, S. Brunet, J.-L. GuYot, « Amélioration de l'attractivité et de la compétitivité du territoire wallon pour le secteur des services supérieurs : étude stratégique exploratoire», Rapport de recherche de l'IWEPS, $\mathrm{n}^{\circ}$ 14, 2015. 
des activités de base pour la compétitivité des territoires. Par conséquent, il ne s'agit plus de simples activités induites; certains auteurs vont même jusqu'à considérer que "ce ne sont pas les producteurs de services qui doivent s'implanter à proximité des utilisateurs de ces services, mais l'inverse ${ }^{21}$.

L'analyse des mécanismes mis en œuvre par les économies d'agglomération a également conduit la littérature à établir une distinction entre les économies de localisation (ou de juxtaposition) et les économies d'urbanisation. Le concept d'économie de localisation renvoie aux avantages que les firmes retirent d'une localisation proche de firmes du même secteur. Les exemples d'agglomération de firmes du même secteur sont légion, du « sentier » parisien à Hollywood, en passant par la sidérurgie liégeoise, le textile courtraisien ou le diamant anversois. En opposition au concept d'économie de localisation, le concept d'économie d'urbanisation rend compte des avantages accessibles à des producteurs qui ont comme seul lien apparent une localisation au sein d'une vaste zone urbaine. Ces économies tiennent à l'accès à de grandes infrastructures (ports ou aéroports), à l'apport d'équipements culturels ou éducatifs, à la proximité de firmes spécialisées qui offrent leurs services à une clientèle diversifiée d'entreprises, ainsi qu'au partage, nous l'avons déjà écrit, d'une main-d'œuvre abondante et bien formée.

L'importance des économies d'urbanisation pousse à revenir sur le sujet des infrastructures de transports et des accessibilités. La révolution des transports s'est traduite par une amélioration globale des accessibilités. Pour autant, ce sont en réalité les principaux nœuds de communication - les hubs - qui en ont le plus bénéficié. Cela s'explique par l'évolution des systèmes techniques, où l'accroissement des vitesses et des capacités conduit à limiter le nombre de nœuds d'accès. Il en résulte un renforcement de l'accessibilité des grandes villes ainsi que la multiplication des effets «tunnels ", c'està-dire des situations où un espace traversé par un axe de transport ne peut accéder à celui-ci. Ces effets tunnels tiennent au déploiement des réseaux de train à grande vitesse, mais ils tiennent surtout au développement du transport aérien. Vu l'intensification des relations internationales dans la vie des affaires, ce mode de transport occupe désormais une place prépondérante dans l'organisation de l'économie. Il est donc souvent crucial pour les entreprises de ne pas trop s'éloigner d'un hub aéroportuaire. Dans cette perspective, il n'est guère surprenant que l'évolution des systèmes de transport soit aussi un facteur fréquemment mis en avant afin d'expliquer la métropolisation.

\subsubsection{La métropolisation}

Les économies d'agglomération étant particulièrement prégnantes au sein des plus grandes villes, il n'est pas si étonnant que ces dernières prennent une part croissante dans l'économie. Que ce soit en termes d'externalités d'informations, d'externalités liées aux marchés du travail ou d'externalités liées à la présence de services spécialisés et de centres de recherche, les grandes villes bénéficient d'avantages cumulatifs qui induisent une polarisation de la vie économique. Cette polarisation vers les grands pôles est aujourd'hui communément dénommée par le terme de métropolisation. Ce terme a émergé dans

S. ILLERIS, «Quels rôles pour les services dans le développement régional ? », in C. GALLOU et al. (dir.), Services aux entreprises et développement régional, Bruxelles, De Boeck, 2005, p. 55. 
les années $1980^{22}$, lorsque le mouvement de croissance des grandes régions urbaines est reparti de plus belle après s'être interrompu durant les décennies 1960 et 1970 (d'abord en raison de la décentralisation industrielle et ensuite en raison des crises économiques).

La métropolisation tient au fait que les grandes villes soient bien armées pour faire face à l'internationalisation et à la mondialisation de l'économie. Elles seules possèdent la gamme variée des services aux entreprises et elles seules disposent des réseaux et des nœuds de transport permettant de capter les flux et d'organiser les échanges. Il est communément admis que la taille est une condition nécessaire - mais bien sûr pas suffisante - à l'émergence du dynamisme métropolitain. Derrière cette idée se cache le fait que les économies d'agglomération sont proportionnelles à la taille des aires urbaines. S'agissant des externalités liées aux marchés du travail, nous pouvons illustrer notre propos avec les résultats d'une étude récente sur les villes américaines. Cette étude montre que le doublement de la taille des bassins d'emploi s'accompagne d'une progression moyenne de $10 \%$ de l'indicateur du PIB par habitant ${ }^{23}$. Sur le même sujet, Philippe McCann et Zoltan J. Acs montrent qu'un potentiel de 1,5 million à 2 millions de personnes est nécessaire pour répondre aux besoins qualitatifs en main-d'œuvre des multinationales des secteurs de la connaissance ${ }^{24}$.

La métropolisation qui se met en place dans les années 1980 conduit de nombreux observateurs à souligner le rôle moteur des villes sur le développement économique régional. En France, c'est alors ce qui pousse la Délégation interministérielle à l'aménagement du territoire et à l'attractivité régionale (DATAR) ${ }^{25}$ à financer l'étude de Roger Brunet sur le positionnement des villes françaises dans la coopération et la concurrence internationale ${ }^{26}$. Cette étude conduit à populariser l'expression de «banane bleue ", afin de décrire la dorsale européenne qui, du Lancashire à la Toscane en passant par la Belgique, concentre les plus fortes densités de grandes villes ainsi que les plus fortes productions et valeurs ajoutées au kilomètre carré. Avec la dorsale européenne, la ressemblance est frappante entre la géographie du fait urbain et la géographie de la production de richesse. Pour l'expliquer, nous pouvons constater qu'une localisation le long de cette dorsale s'accompagne automatiquement de la proximité avec des pôles urbains importants, proximité dont nous savons qu'elle s'accompagne de grands avantages pour la compétitivité des firmes. Pour étayer ce constat, faisons référence aux analyses qui montrent que les villes françaises de taille moyenne (les aires urbaines de 50000 à 200000 habitants) résistent mieux à la désindustrialisation lorsqu'elles se situent à moins de 60 kilomètres d'une grande ville (les aires urbaines de plus de 200000 habitants) ${ }^{27}$.

22 P. Claval, « Métropolisation et globalisation », Géographie et culture, volume 48, 2003, p. 5-24.

S. ANGEL, A. M. BLEI, "Commuting and the productivity of American cities », Working Paper NYUMarron Institute of Urban Management, $\mathrm{n}^{\circ}$ 19, 2015.

24 P. McCANn, Z. J. ACS, "Globalization: Countries, cities and multinationals», Regional Studies, volume $45, \mathrm{n}^{\circ} 1,2011$, p. 17-32. La situation du développement articulé par Luxembourg-Ville apparaît, à proximité immédiate de la Wallonie, comme une exception notable à la règle générale qui relie métropolisation et taille des bassins d'emplois. Cet écart à la règle s'explique par les niveaux de rémunération particulièrement élevés qui sont pratiqués au Grand-Duché de Luxembourg pour les emplois métropolitains. En effet, ces niveaux de rémunération exceptionnels signifient qu'un emploi offert au Grand-Duché sera attractif pour des populations spatialement très éloignées.

5 Administration de mission française créée en 1963 et aujourd'hui appelée le Commissariat général à l'égalité des territoires (CGET).

26 R. BRUNET, Les villes européennes, Paris, DATAR / La Documentation française, 1989.

27 V. FouchIER, «Tendances longues de l'évolution économique des métropoles françaises. Un regard critique sur la notion de taille critique », Territoires $2030, \mathrm{n}^{\circ} 1,2005, \mathrm{p} .40$. 
Les chercheurs français ne sont pas les seuls à convenir du fait que le dynamisme économique des villes soit fondamental pour assurer la compétitivité des territoires et des régions. Suivons ici Michael Parkinson et ses collègues britanniques lorsque, après leur tour d'Europe des régions compétitives, ils dressent la conclusion suivante : «Les régions les plus compétitives sont les régions qui possèdent les villes les plus compétitives. Par contre, nous n'avons pas pu identifier de régions gagnantes où ne se trouvent pas de villes gagnantes ${ }^{28}$.

Ce constat est interpellant vis-à-vis de la Wallonie. Nous l'avons déjà souligné, le territoire régional n'héberge pas de réelles métropoles qui seraient des hauts lieux de polarisation de la vie économique. Pour autant, comme nous le détaillerons, des parties importantes du territoire wallon subissent fortement l'impact des dynamiques insufflées par des pôles métropolitains extérieurs.

Sans conteste, le pôle métropolitain extérieur qui a le plus fort impact sur la Wallonie est celui de Bruxelles. Cette réalité a été intégrée par la politique d'aménagement du territoire des autorités wallonnes dès 1999 , avec le projet de structurer le "Triangle wallon ${ }^{29}$. La mise en exergue du Triangle wallon vise alors à créer le pendant du «Diamant flamand", un concept formalisé en 1997 par les autorités flamandes ${ }^{30}$. Cet espace en forme de losange correspond à la zone sous l'influence des villes d'Anvers, Bruxelles, Gand et Louvain; il forme le cœur urbain de la Flandre. Le Triangle wallon est donc une réponse en termes de structuration existante et souhaitée de la Wallonie. Il se base sur le constat de l'influence de Bruxelles - notamment en termes de navettes quotidiennes - sur une grande partie de la Wallonie. Le Triangle wallon, avec en son cour le Brabant wallon, est approximativement borné par un triangle qui relie Bruxelles, Mons et Namur et dont la base passe par Charleroi.

La mise en exergue des concepts du Diamant flamand et du Triangle wallon s'inscrit dans la volonté des autorités respectivement flamandes et wallonnes de maximiser les retombées des logiques métropolitaines bruxelloises. Cette mise en exergue témoigne du rôle central joué par Bruxelles dans la structuration économique de la Belgique en général et de la Wallonie en particulier ${ }^{31}$. Au niveau wallon, il convient toutefois de souligner l'hétérogénéité du Triangle wallon entre, au Nord, le Brabant wallon qui bénéficie fortement de sa proximité à Bruxelles et, au Sud, une nébuleuse de tradition industrielle qui s'étend de l'Ouest namurois à Mons ${ }^{32}$.

28 "The most competitive regions also had the most competitive cities. Conversely we found no examples of successful regions which had unsuccessful cities at their core» (M. PARKINSON et al., Competitive European cities: Where do the core cities stand?, Londres, Office of the Deputy Prime Minister, 2004, p. 53).

29 Gouvernement wallon, «Schéma de développement de l'espace régional », Namur, Ministère de la Région wallonne, 1999.

30 «Ruimtelijk Structuurplan Vlaanderen », Bruxelles, Ministerie van de Vlaamse Gemeenschap, 1997. L. AUJEAN et al., "Le positionnement des villes belges dans le réseau global des services avancés », Belgeo. Revue belge de géographie, $\mathrm{n}^{\circ}$ 1, 2007, https://journals.openedition.org; J.-F. THISSE, I. THOMAS, "Bruxelles et Wallonie : une lecture en termes de géo-économie urbaine », Reflets et perspectives de la vie économique, volume 46, $\mathrm{n}^{\circ}$ 1, 2007, p. 75-93; M. VAN MEETEREN et al., «Flemish diamond or ABC-axis? The spatial structure of the Belgian metropolitan area », European Planning Studies, volume 24, n 5 , 2016, p. 974-995.

32 M. VAN CRIEKINGEN, P. CORNUT, S. LUYTEN, « Brussels: polycentricity as “images on the map", not in reality », in N. CATTAN (dir.), Cities and network in Europe. A critical approach of polycentrism, Paris, John Libbey Eurotext, 2007, p. 105-112. 


\subsubsection{Typologie des flux de revenus}

Comme mentionné dans l'introduction générale de ce Courrier hebdomadaire, nous faisons référence à la notion de ressource territoriale pour rendre compte des caractéristiques des territoires qui permettent d'y attirer des flux de revenus. Dans cette sous-section, nous allons maintenant constater que les flux de revenus qui irriguent les territoires peuvent être très diversifiés.

Les éléments développés sur les économies d'agglomération et sur la métropolisation ne correspondent qu'à un modèle de développement économique régional : un modèle basé sur un secteur productif local capable de générer des revenus grâce à des exportations en dehors du territoire. Pour compléter notre vision, il est important d'également considérer le rôle de l'économie résidentielle et présentielle, qui est devenue vitale au sein de très nombreux territoires où le bien-être des populations ne peut uniquement dépendre des exportations du secteur productif. C'est dans ce cadre que nous avons choisi de nous appuyer sur le tableau 1. Ce tableau, adapté des travaux de Delphine Guex et Olivier Crevoisier ${ }^{33}$, établit une typologie des flux de revenus en quatre types. La typologie en question se base sur une double approche qui différencie, d'une part, le territoire comme lieu de production et le territoire comme lieu de consommation et, d'autre part, la demande locale et la demande extra-locale.

Tableau 1. Typologie des flux de revenus qui bénéficient aux territoires

\begin{tabular}{|c|c|c|c|}
\hline & \multicolumn{2}{|c|}{ Dimension de la demande pour le territoire } \\
\hline & & Demande extra-locale & Demande locale \\
\hline \multirow{2}{*}{ 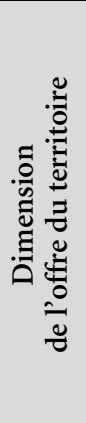 } & $\begin{array}{l}\text { Le territoire comme lieu } \\
\text { de production }\end{array}$ & $\begin{array}{c}\text { Type « fabrication » } \\
\text { Production pour les marchés } \\
\text { d'exportation de biens } \\
\text { et de services } \\
\text { Développement } \\
\text { par la compétitivité industrielle } \\
\text { et servicielle }\end{array}$ & $\begin{array}{l}\text { Type « circuits courts» } \\
\text { Production à l'attention } \\
\text { de la population locale } \\
\text { Développement } \\
\text { par la substitution } \\
\text { aux importations }\end{array}$ \\
\hline & $\begin{array}{l}\text { Le territoire comme lieu } \\
\text { de consommation }\end{array}$ & $\begin{array}{l}\text { Type «tourisme-navettes" } \\
\text { Attractivité des consommateurs } \\
\text { (shopping, loisirs, vacances, etc.) } \\
\text { Développement par l'attractivité } \\
\text { résidentielle et présentielle }\end{array}$ & $\begin{array}{c}\text { Type « services locaux » } \\
\text { Économie liée aux besoins } \\
\text { locaux de la population } \\
\text { Développement } \\
\text { par la diversification interne }\end{array}$ \\
\hline
\end{tabular}

Source: adapté de D. GuEX, O. CrevoISIER, "Globalisation postindustrielle et milieu locaux : une typologie », Working Paper MAPS, $n^{\circ} 1,2017$.

Les flux de revenus «fabrication» s'inscrivent dans la logique de l'économie productive. Le territoire est ici intégré dans les continentalisation et globalisation de l'économie par l'exportation de biens et de services. Le type "fabrication » est étroitement lié aux conceptions à l'origine de la théorie de la base ${ }^{34}$. Selon cette théorie classique, une région tire son développement des revenus «basiques » des firmes exportatrices situées sur son territoire. Dans la théorie de la base, grâce au multiplicateur économique, les revenus

33 D. Guex, O. Crevolsier, «Globalisation postindustrielle et milieux locaux : une typologie », op. cit. ;

R. CAMAGNI, "Attractivité et compétitivité, un binôme à repenser », op. cit., p. 11-15.

J. W. AleXANDER, "The non-basic concept of urban economic functions», Economic geography, volume $30, n^{\circ} 3,1954$, p. 246-261. 
basiques alimentent en revenus les secteurs non basiques (également appelés domestiques). Dans le tableau 1, cela renvoie au type «services locaux». Historiquement, la complémentarité entre la «fabrication » et les «services locaux» tient au fait que les revenus induits par les exportations ont permis le développement d'une offre locale de services aux personnes, dans des domaines aussi variés que le commerce de détail, la santé, l'éducation, etc.

Le type «tourisme-navette » fait référence à la question de l'économie résidentielle et présentielle. Nous sommes ici en présence de flux de revenus liés à des activités productives situées ailleurs : ailleurs dans le temps avec les retraités, et ailleurs dans l'espace avec les touristes, les résidents actifs travaillant à l'extérieur et les bénéficiaires des prestations sociales. La conception à l'origine des travaux sur l'économie présentielle repose sur l'idée qu'il faut élargir la notion de base économique : à côté de la base productive (assimilée aux entreprises exportatrices), il faut aussi considérer la base présentielle. La base présentielle englobe notamment la base résidentielle avec les personnes qui résident sur le territoire. Cette base résidentielle est alimentée par les revenus des résidents actifs travaillant à l'extérieur, mais aussi par divers types de revenus distribués par les autorités publiques: les salaires des fonctionnaires, les retraites des pensionnés, les prestations sociales des allocataires sociaux et les remboursements des frais médicaux ${ }^{35}$. En plus de la base résidentielle, la base présentielle doit également tenir compte des personnes qui sont présentes sur le territoire sans y résider de façon permanente. En effet, diverses catégories d'acteurs - les touristes et les excursionnistes mais également les travailleurs-navetteurs et les étudiants - sont susceptibles de contribuer à une économie locale sans y résider.

Le quatrième type de flux de revenus repris dans le tableau 1 correspond à la catégorie « circuits courts ». Nous sommes ici dans une logique de réduction des importations, avec le développement de circuits courts où des productions locales destinées aux populations locales tendent à remplacer des circuits d'importation depuis l'extérieur du territoire. À l'heure actuelle, le potentiel de développement des flux de revenus liés aux circuits courts demeure difficile à évaluer ${ }^{36}$. Nous pouvons toutefois imaginer qu'il est conséquent au regard de son importance dans la perspective des transitions écologique et énergétique. En effet, le développement de cette catégorie de flux de revenus devrait s'accompagner d'une limitation des transports et, dès lors, d'une limitation des consommations énergétiques et des externalités environnementales. Pour les territoires, le caractère stratégique des circuits courts est également à mettre en parallèle avec le scénario possible d'une démondialisation qui entraînerait une stagnation voire une réduction du commerce international ${ }^{37}$.

35 C. CRAgUe, «Le maintien des entreprises, un problème d'urbanisme. L'équilibre fonctionnel comme principe de l'intervention économique locale ", Revue internationale d'urbanisme, $\mathrm{n}^{\circ}$ 4, 2017.

L'Institut wallon de l'évaluation, de la prospective et de la statistique (IWEPS) avait fait de la mesure de ces dynamiques d'économie circulaire et ancrée dans le territoire l'objet de sa conférence en 2016, preuve des défis méthodologiques à mesurer ces flux économiques.

F. LIVESEY, From global to local: the making of things and the end of globalisation, Londres, Profile Books, 2017. 


\subsubsection{La périurbanisation}

Métropolisation et périurbanisation sont deux tendances à forte dimension géographique qui sont parfois confondues et amalgamées. Pourtant, il est important de les distinguer au regard des deux grandes différences qui les opposent.

La première différence est que la métropolisation et la périurbanisation ne se développent pas aux mêmes échelles. La métropolisation doit s'appréhender aux niveaux spatiaux auxquels l'économie productive se structure. En fonction des types de produits et services, cette structuration s'opère de l'échelle régionale à l'échelle mondiale en passant par les échelles des États et des continents. Pour sa part, la périurbanisation est à appréhender à l'échelle des bassins de vie ou des aires urbaines fonctionnelles, deux notions qui renvoient à l'étendue territoriale à l'intérieur de laquelle les populations mènent leurs activités quotidiennes : se loger, travailler, se former, se divertir, etc. La périurbanisation se développe d'ailleurs tant au sein des aires urbaines des métropoles qu'au sein des aires urbaines articulées par des villes moyennes ou même petites.

La seconde différence majeure entre métropolisation et périurbanisation est que la première est un phénomène de polarisation et de concentration alors que la seconde est, à l'inverse, un phénomène de diffusion et de déconcentration. Nous l'avons précisé cidessus, la métropolisation est une polarisation de l'activité économique vers les grands pôles urbains. Par contre, la périurbanisation est un phénomène de déconcentration des populations et des activités qui se produit aux dépens des territoires centraux des aires urbaines et au bénéfice de leurs territoires plus périphériques (qui peuvent d'ailleurs englober de petites villes).

Si les notions de métropolisation et de périurbanisation sont à différencier, leurs deux grilles de lecture peuvent être conjuguées afin d'analyser les évolutions territoriales. Par exemple, ces deux tendances structurelles interagissent pour contribuer à la vitalité du Brabant wallon, qui bénéficie tant des dynamiques de concentration vers la zone métropolitaine de Bruxelles que des dynamiques de déconcentration depuis les parties les plus centrales de cet espace.

L'évolution vers la périurbanisation résulte de causes multiples, que nous ne pouvons pas développer ici de manière approfondie ${ }^{38}$. Retenons toutefois que son facteur déclenchant correspond à l'impact du relâchement des contraintes de mobilité et de la banalisation de la mobilité routière sur les choix et arbitrages résidentiels ${ }^{39}$. De nombreux travaux relèvent que la périurbanisation résidentielle est rendue possible car l'accroissement des vitesses de déplacement permet aux populations d'accroître l'extension spatiale de leurs bassins de vie tout en maintenant constant le temps journalier qu'ils consacrent aux déplacements. À l'image des localisations des ménages, les localisations des entreprises ont aussi très fortement évolué suite aux mutations des mobilités dans les aires urbaines. Les logiques de production économique ont donc également contribué à la périphérisation massive des composantes de la ville, notamment via le déploiement spatial de la grande distribution, mais également via la formule du parc d'activités économiques périphérique,

38 Cf., par exemple, J.-M. HALleux, «Vers la ville compacte qualitative ? Gestion de la périurbanisation et actions publiques », Belgeo. Revue belge de géographie, $\mathrm{n}^{\circ}$ 1-2, 2012, https://journals.openedition.org. M. WIEL, La transition urbaine ou le passage de la ville pédestre à la ville motorisée, Sprimont, Mardaga, 1999. 
qui permet d'offrir du foncier bon marché aux entreprises tout en évitant que les populations des noyaux urbains subissent les nuisances des secteurs lourds.

La périurbanisation, que l'on appelle aussi parfois l'étalement urbain, ne va pas sans poser un certain nombre de problèmes, en particulier en Belgique où elle est très développée et particulièrement consommatrice d'espace. La périurbanisation est préjudiciable car elle s'accompagne de surcoûts en infrastructures ${ }^{40}$ et en consommations énergétiques liées aux trafics routiers ${ }^{41}$. En outre, elle est aussi très problématique en raison de son impact négatif sur les noyaux urbains. En Wallonie, les fortes périurbanisations résidentielles et économiques accentuent les problèmes de la multiplication des friches et de la paupérisation des cœurs de ville. Aujourd'hui, alors que la qualité des espaces urbains représente un levier de plus en plus reconnu de l'attractivité et de la compétitivité des territoires, ces évolutions sont dommageables pour l'attractivité globale du territoire régional ${ }^{42}$.

\subsubsection{Attractivité, productivité et compétitivité}

L'attractivité territoriale peut se définir comme la capacité d'un territoire à attirer et à retenir des générateurs de revenus. Dans l'économie productive, la question de l'attractivité se pose pour l'attraction et le maintien des entreprises, mais également pour l'attraction et le maintien des facteurs de production mobiles que sont les capitaux, les équipements et les travailleurs qualifiés.

La question de l'attractivité des territoires renvoie à la question des investissements directs étrangers (IDE). Les IDE sont classiquement définis comme « une activité par laquelle un investisseur résidant dans un pays obtient un intérêt durable et une influence significative dans la gestion d'une entité résidant dans un autre pays. Cette opération peut consister à créer une entreprise entièrement nouvelle (investissement de création - greenfield investment) ou, plus généralement, à modifier le statut de propriété des entreprises existantes par le biais de fusions et d'acquisitions $»{ }^{43}$.

Les IDE représentent une part croissante de la production ${ }^{44}$ et ils constituent l'une des principales composantes des continentalisation et mondialisation de l'économie. Dans un environnement économique où les territoires sont de plus en plus en concurrence, ils sont devenus un enjeu majeur pour le développement de l'économie productive, en particulier en Wallonie ${ }^{45}$. La problématique des IDE pose la question de la capacité à attirer

40 J.-M. Halleux, J.-M. LAmbOtTe, L. BRÜCK, «Étalement urbain et services collectifs : les surcoûts d'infrastructure liés à l'eau ", Revue d'économie régionale et urbaine, volume 21, $\mathrm{n}^{\circ}$ 1, 2008, p. 21-42.

41 C. BAZET-SimONi et al., «Face à l'épuisement du pétrole, quel rôle pour l'aménagement du territoire en Wallonie? ", Regards économiques, $\mathrm{n}^{\circ}$ 87, 2011.

42 B. Bianchet, D. Claeys, J. Descamps, C. MerCenier, C. Ruelle, R. SANDu, Politique de la ville. Renforcement des centralités urbaines et de leur attractivité sous l'angle économique, Namur, CPDT, 2017 ; P.-F. WILMOTTE, C. RuelLe, B. BIANCHET, « Les enjeux de l'investissement privé en milieu urbain : l'exemple de la Wallonie », Bulletin de la Société géographique de Liège, volume 71, n 2, 2018.

3 «Tendances de l'investissement direct étranger dans les pays de l'OCDE », Perspectives économiques de l'OCDE, $\mathrm{n}^{\circ} 73,2003, \mathrm{p} .193$.

44 B. MÉRENNE-SCHOUMAKER, «La Belgique : une économie sous influence étrangère », Belgeo. Revue belge de géographie, $\mathrm{n}^{\circ} 4,2017$, https://journals.openedition.org.

F. Collard, C. Goethals, M. Wunderle, "L'actionnariat des entreprises en Wallonie en 2015 ", Courrier hebdomadaire, CRISP, $\mathrm{n}^{\circ} 2329,2017$. 
les entreprises, mais également de la capacité à les retenir. Certains exemples récents l'ont malheureusement démontré (en particulier Caterpillar et ArcelorMittal).

Pour les territoires, le renforcement de l'attractivité pour les entreprises passe par une capacité à offrir des ressources spécifiques et spécialisées ${ }^{46}$. Bernard Pecqueur parle ici d' " avantages différenciatifs », qui permettent le développement d'un appareil productif spécialisé dans des filières spécifiques ${ }^{47}$. Cette notion de l'avantage différenciatif recoupe la notion d'économie de localisation (ou de juxtaposition) précédemment définie.

Dans l'économie productive, l'attractivité est fortement dépendante de la productivité, c'est-à-dire du rapport entre la production de valeur ajoutée et la quantité des facteurs de production mobilisés (par exemple, le temps de travail). Comme le résume Roberto Camagni, les capitaux et les facteurs de production « se dirigent vers les territoires où les coûts sont mineurs (attractivité par les prix) ou bien vers les territoires où les coûts supérieurs sont plus que compensés par une forte productivité (attractivité par la productivité) ${ }^{48}$.

L'attractivité est à considérer pour l'économie productive, mais aussi pour l'économie résidentielle et présentielle. L'enjeu consiste alors à rendre le territoire attractif pour que l'on y dépense des revenus générés ailleurs. Cela peut se produire par des navettes domicile-travail ou par des déplacements liés aux commerces de détail, aux loisirs, aux études ou aux voyages touristiques. Lorsque l'on cherche à développer une économie présentielle en renforçant l'attractivité, cela peut se répercuter positivement sur l'économie productive suite à l'amélioration de l'attrait des lieux pour les mains-d'œuvre qualifiées ; or celles-ci constituent une ressource territoriale de plus en plus vitale pour les entreprises dans le contexte de l'économie de la connaissance (cf. supra, 1.2.1 à 1.2.4). Cette observation souligne les interconnexions entre l'économie présentielle et l'économie productive, qui ressortent comme deux composantes complémentaires d'un développement territorial équilibré.

Lorsque la notion de compétitivité est appliquée aux entreprises, elle désigne leur capacité à faire face à la concurrence. Pour une entreprise, faire face à la concurrence est possible via une compétitivité en prix, lorsqu'elle peut proposer, pour un produit ou un service équivalent, des prix inférieurs à ceux de ses concurrents. On parle également de compétitivité hors prix, lorsque les producteurs imposent leurs produits par des facteurs autres que le prix : la qualité, l'innovation, l'image de marque, le mode de commercialisation, les services associés, etc.

En plus de s'appliquer aux entreprises, la notion de compétitivité s'applique désormais, par extension, aux territoires. La notion de compétitivité territoriale a ici évolué d'une perspective centrée sur les échanges extérieurs à une perspective plus globale d'amélioration du niveau de vie et du bien-être social des populations ${ }^{49}$. Un territoire compétitif devient alors un territoire capable d'atteindre les objectifs fixés par les stratégies de développement.

46 P. NiJKAmP, "The resourceful region: a new conceptualisation of regional development strategies ", Investigaciones regionales: Journal of regional research, $n^{\circ} 36,2016, \mathrm{p} .191-214$.

B. PECQUEUR, "L'économie territoriale : une autre analyse de la globalisation ", L'Économie politique, volume $33, \mathrm{n}^{\circ} 1,2007, \mathrm{p} .41-52$.

48 R. CAMAGNI, «Attractivité et compétitivité, un binôme à repenser », op. cit., p. 12.

49 B. MÉRENNE-SCHOUMAKER, «De la compétitivité à la compétence des territoires. Comment promouvoir le développement économique ? Exposé de synthèse », Territoire(s) wallon(s), hors-série n 1, 2007, p. 51-58. 
Par exemple, une région européenne est jugée compétitive lorsqu'elle rencontre les objectifs que la Commission européenne formalise via sa stratégie Europe 2020 (cf. infra, 2.1.1). À la suite de la stratégie de Lisbonne, la stratégie Europe 2020 affiche notamment des objectifs pour la cohésion sociale, les niveaux d'emploi et la recherche et développement $(\mathrm{R} \& \mathrm{D})$.

\section{LES RESSOURCES TERRITORIALES}

Rappelons que nous avons défini les ressources territoriales comme étant les caractéristiques des territoires qui permettent d'y attirer des flux de revenus. Sur la base de la section précédente, les ressources territoriales sont à appréhender comme les sources de la compétitivité des territoires et de leur attractivité. Afin de développer la grille de lecture des ressources territoriales, il est utile de détailler certaines d'entre elles jugées aujourd'hui essentielles. Les thématiques que nous allons passer en revue dans ce cadre sont les suivantes : le facteur connaissance et les systèmes régionaux d'innovation, la « classe créative » comme facteur d'attractivité des entreprises, le capital humain et le déficit d'attractivité des RETI, les aménités ${ }^{50}$ et la qualité des lieux et, in fine, les facteurs matériels des infrastructures de transport et du foncier à vocation économique.

\subsubsection{Le facteur connaissance et les systèmes régionaux d'innovation}

Nous avons vu que la productivité est devenue un déterminant clé de l'attractivité et de la compétitivité des territoires. La productivité des firmes est largement déterminée par l'innovation qui, avec la montée en puissance du facteur connaissance, est devenue un enjeu stratégique pour les entreprises ${ }^{51}$. Par ailleurs, les villes et les territoires sont de plus en plus reconnus comme des catalyseurs potentiels pour la valorisation des connaissances, comme l'illustre le succès des théories économiques qui soulignent le rôle des réseaux territorialisés dans la capacité des entreprises à innover. Les publications scientifiques parlent ici des milieux innovateurs, des régions apprenantes ou des clusters $^{52}$, des notions que nous choisissions de traiter à travers le concept générique de système régional d'innovation (SRI). Ce concept de SRI fait référence «à des concentrations spatiales d'entreprises et d'organisations publiques et semi-publiques (universités, instituts de recherche, agences de transfert et liaison technologique, associations d'affaire, organismes gouvernementaux, etc.) qui produisent de l'innovation sur la base d'interactions et d'apprentissage collectif au travers de pratiques institutionnelles communes ${ }^{53}$.

50 Par aménité, il faut entendre un aspect de l'environnement appréciable et agréable, dans un lieu ou site particulier.

51 R. L. MARTIN, A study on the factors of regional competitiveness. A draft final report for the European Commission, Cambridge, University of Cambridge / Directorate-General Regional Policy, 2003.

52 D. Maillat, M. QuÉviT, L. SENN (dir.), Réseaux d'innovation et milieux innovateurs: un pari pour le développement régional, Neufchâtel, GREMI, 1993 ; R. FLORIDA, " Toward the learning region », Futures, volume $27, n^{\circ} 5,1995$, p. 527-536; M. PORTER, «Clusters and the new economy of competition », op. cit.,

p. $77-90$

D. Doloreux, S. Dionne, «Le système régional d'innovation dans la périphérie : observations, synthèse et interrogations ", Territoire(s) wallon(s), hors-série $\mathrm{n}^{\circ}$ 1, 2007, p. 105. 
Le développement des SRI repose sur des réseaux d'acteurs ancrés dans le territoire grâce à un capital social qui facilite le partage d'informations et les collaborations entre agents économiques ${ }^{54}$. Largement popularisée auprès des autorités publiques, la littérature sur les liens entre systèmes régionaux d'innovation et développement économique a conduit à la mise en œuvre des politiques de clustering, qui visent à stimuler ces collaborations et partages d'informations. Comme indiqué ci-dessus à propos des économies d'agglomération liées à la circulation des informations, la politique wallonne n'est pas en reste dans ce domaine, avec les outils du cluster et du pôle de compétitivité ${ }^{55}$.

Il existe en Wallonie six pôles de compétitivité. Ils couvrent les domaines suivants : les biotechnologies (BioWin), la logistique (Logistics in Wallonia), l'aéronautique et le spatial (SkyWin), le génie mécanique (Mecatech), l’agro-alimentaire (Wagralim) et les technologies vertes (Greenwin). Les pôles de compétitivité sont essentiellement soutenus pour développer des projets d'investissement, de R\&D ou de formation. L'outil du cluster est complémentaire à l'outil du pôle de compétitivité. Il s'agit de structures plus légères, dont l'objectif est de stimuler l'animation économique et de soutenir les mises en réseau autour de thématiques communes pour, à terme, générer de nouveaux partenariats. Nous aurons l'occasion de revenir plus en détail sur les pôles de compétitivité au point 4.1.4, dédié à la géographie de leurs membres.

En dépit de leur large mise en application, les recherches sur les SRI manquent de clarté sur trois points principaux, qui concernent pourtant le caractère opérationnel des politiques de clustering. D'abord, il n'existe pas de réel consensus scientifique sur l'échelle pertinente pour mener ce type de politiques. En effet, la littérature souffre d'un manque de précision quant à la manière dont ces systèmes s'organisent et se structurent dans l'espace ${ }^{56}$. Ensuite, les chercheurs constatent que la seule proximité géographique ne suffit pas à créer des SRI performants et les études continuent d'interroger le rôle d'autres formes de proximité, à l'image des proximités sociales ou intellectuelles ${ }^{57}$. Enfin, et il s'agit là d'un point crucial, rien n'indique à ce jour que le territoire où une innovation voit le jour va devenir le territoire bénéficiaire lorsque l'exploitation de l'innovation en question va générer de nouveaux revenus ${ }^{58}$.

54 M. WOOLCOK, D. NAVARAN, «Social capital: implications for development theory, research, and policy », The World Bank research observer, volume 15, $\mathrm{n}^{\circ} 2,2000$, p. 225-249 ; C. PERRET, "Capital social et développement territorial », Notes de recherches de l'IREGE, $\mathrm{n}^{\circ}$ 11-01, 2011.

55 À ce propos, cf. les livraisons du Courrier hebdomadaire consacrées à la question, parmi lesquelles : A. ACCAPUto, B. BAYENET, G. PAgAnO, «Le Plan Marshall pour la Wallonie », Courrier hebdomadaire, CRISP, n 1919-1920, 2006 ; C. Dujardin, P. LefebVRe, M. LefèVRe, V. Louis, F. PAllez, F. VANDERKelen, «Les pôles de compétitivité wallons : dix ans de politique industrielle», Courrier hebdomadaire, CRISP, $\mathrm{n}^{\circ}$ 2337-2338, 2017 ; C. Dujardin, P. Lefebrve, M. Lefèvre, V. Louis, F. Pallez, F. Vanderkelen, «L'évaluation des pôles de compétitivité wallons : méthodes, résultats et mise en perspective européenne ", Courrier hebdomadaire, CRISP, $\mathrm{n}^{\circ}$ 2339-2340, 2017.

56 P.-F. Wilmotte, J.-M. Halleux, « La structure spatiale des systèmes régionaux d'innovation », op. cit., p. $51-70$.

7 P.-A. BAlland, R. BosChma, K. FrenKEN, « Proximity and innovation: From statics to dynamics », Regional Studies, volume 49, n 6, 2015, p. 907-920.

58 R. SHEARMUR, «Innovation et développement territorial ? L'innovation comme un processus (presque) aterritorial », Bulletin de la Société géographique de Liège, $n^{\circ}$ 55, 2010, p. 17-27. 


\subsubsection{La « classe créative » comme facteur d'attractivité des entreprises}

Par leurs capacités à proposer de nouvelles idées, les individus créatifs font l'objet d'une attention particulière dans la littérature sur l'innovation, comme l'illustre le succès de la théorie de la "classe créative » formalisée par le géographe économique américain Richard Florida ${ }^{59}$. Cette théorie repose sur deux postulats principaux. Le premier est que les entreprises des secteurs économiques les plus porteurs sont attirées vers les villes qui concentrent les talents de la classe créative. Le second est que la classe créative est mobile et qu'elle est attirée vers des lieux spécifiques. Selon R. Florida, ces lieux présentent deux caractéristiques principales. D'une part, il s'agit de lieux de tolérance, où les nouveaux arrivants sont rapidement acceptés et intégrés. D'autre part, il s'agit également de lieux où les aménités urbaines sont très développées, en particulier l'offre culturelle au sens large.

Depuis 2002 et la publication de l'ouvrage The Rise of the Creative Class par R. Florida, la théorie de la "ville créative » a séduit de nombreuses autorités locales. Cela a débuté en Amérique du Nord pour ensuite se diffuser en Europe, y compris en Belgique et en Wallonie $^{60}$. C'est notamment dans cette optique qu'il faut comprendre les grands projets urbains menés dans de nombreuses villes américaines et européennes, avec des opérations où les infrastructures culturelles et les aménités urbaines sont considérées comme des leviers d'attractivité auprès des populations créatives.

Bien qu'inspirante pour de nombreuses autorités locales, la théorie de la classe créative reste décriée par de nombreux chercheurs. Les critiques à son encontre sont principalement de trois ordres. Premièrement, est critiqué le flou qui règne généralement autour de la notion de classe créative ${ }^{61}$. Deuxièmement, les relations de causalité mises en exergue par R. Florida seraient discutables. Par exemple, pour Richard Shearmur, la question n'est pas de savoir s'il existe un lien entre classe créative et croissance, mais de savoir si la présence de la classe créative cause la croissance ou si la croissance attire les migrants créatifs et diplômés ${ }^{62}$. Un troisième type de critique se rapporte aux effets sociaux de l'application de la théorie de R. Florida. En la matière, le fait que certaines villes aient activement cherché à attirer les jeunes hauts salaires de la classe créative a vraisemblablement contribué à y accroître les inégalités sociales ${ }^{63}$.

Sans entrer dans le débat sur la véracité et l'intérêt de la théorie de la classe créative, nous pouvons retenir qu'elle place la ville au centre des interactions entre les individus créatifs et qu'elle souligne le caractère inducteur de la géographie des talents : ceux-ci ne sont plus considérés comme de simples « suiveurs » des entreprises. Cette théorie est ainsi illustrative d'une époque où les aménités urbaines et les industries culturelles et créatives sont de plus en plus considérées comme des ressources territoriales significatives. L'émergence

59 R. FLORIDA, The rise of the creative class: and how it's transforming work, leisure, community and everyday life, New York, Basic Books, 2002.

T. DeRMINE, «Un plan stratégique pour Charleroi », Courrier hebdomadaire, CRISP, n 2060, 2010.

Nous synthétisons ci-dessous une recherche menée sur la situation de la classe créative en Wallonie où le lecteur intéressé pourra trouver les informations relatives à la définition utilisée (cf. infra, 6.1.2).

R. SHEARMUR, «L'aristocratie mobile du savoir et son tapis rouge. Quelques réflexions sur les thèses de Richard Florida ", in D.-G. TREMBLAY, R. TREMBLAY, La compétitivité urbaine à l'heure de la nouvelle économie, Québec, Presses de l'Université du Québec, 2006, p. 285-303.

63 R. CERNICKY, "Le grand entretien. Philip Lawton: La "ville créative" augmente les inégalités sociales ", Dérivations, $\mathrm{n}^{\circ}$ 4, 2017, p. 16-28. 
de ce paradigme de l'économie créative et culturelle a d'ailleurs conduit au développement de politiques spécifiques, à l'image du programme Creative Wallonia et de la stratégie Digital Wallonia (cf. infra).

\subsubsection{Le capital humain et le déficit d'attractivité des RETI}

La thématique de la ressource territoriale du capital humain présente des points communs avec la théorie de la classe créative. Dans les deux cas, c'est l'enjeu de la qualité de la main-d'œuvre qui est mis en exergue. Une différence importante entre les deux sujets est que l'approche par le capital humain porte surtout sur les qualifications des populations locales. Par contre, la théorie de la classe créative souligne principalement l'enjeu de l'attraction d'une catégorie de professionnels qualifiés supposés très mobiles.

La ressource territoriale du capital humain est généralement appréhendée à travers des indicateurs sur les niveaux de qualification. Par exemple, parmi les indicateurs utilisés par la Commission européenne pour évaluer la compétitivité des régions des États membres de l'Union européenne, on trouve la part des diplômés de l'enseignement supérieur. Cela s'explique par le fait que ce niveau de diplôme favorise tant l'insertion dans les marchés du travail que la propension à l'entrepreneuriat ${ }^{64}$.

La problématique du capital humain est particulièrement aiguë dans les régions européennes de tradition industrielle (RETI). Comme l'a par exemple montré Thomas Dermine à propos de Charleroi ${ }^{65}$, les RETI sont souvent peu attractives pour les jeunes diplômés. Durant des décennies, leur prospérité a reposé sur un système de production basé sur l'abondance d'une main-d'œuvre peu qualifiée. Il s'agit là de l'origine d'un cercle vicieux enclenché par l'inadéquation du capital humain aux besoins de l'économie de la connaissance. Génération après génération, ce cercle vicieux y est entretenu par une spirale d'exodes des jeunes les plus qualifiés et les plus entreprenants. Cette situation illustre les liens qu'entretiennent l'économie productive et l'économie présentielle. Elle témoigne également de la nécessité d'intervenir simultanément sur les deux volets afin de briser les cercles vicieux du déficit d'attractivité territoriale qui caractérise les RETI.

\subsubsection{Les aménités et la qualité des lieux}

Considérer les aménités et la qualité des lieux comme des ressources territoriales se base sur l'idée toute simple que les générateurs de revenus - tant les entreprises que les ménages ont une double propension à privilégier les lieux agréables et à délaisser les lieux désagréables. En la matière, la littérature considère non seulement les aménités naturelles liées au climat ou à l'environnement physique, mais également les aménités patrimoniales ainsi que les infrastructures culturelles et de loisirs ${ }^{66}$.

64 S. MÜLLER, «A progress review of entrepreneurship and regional development: What are the remaining gaps? », European Planning Studies, volume 24, n 6, 2016, p. 1133-1158.

T. DERMINE, «Un plan stratégique pour Charleroi », op. cit.

66 M. PARKINSON et al., Competitive European cities, op. cit. 
Pour les échelons nationaux ou régionaux, le rôle des villes est important en termes d'aménités culturelles et de loisirs. En effet, ce sont des concentrations de populations qui permettent de mutualiser les dépenses liées à de grands équipements. Par exemple, l'Opéra royal de Liège ou le Théâtre de Namur sont des illustrations typiques d'aménités urbaines.

La thématique des aménités en tant que facteur d'attractivité et de compétitivité pose la question des liens entre les domaines du développement régional et de l'urbanisme. En effet, si les générateurs de revenus sont caractérisés par une propension significative à se déplacer vers des lieux agréables, des politiques d'urbanisme visant à mettre en place des aménagements qualitatifs de l'espace se justifient tant vis-à-vis de l'objectif de la croissance économique que vis-à-vis de l'objectif de l'amélioration du bien-être des populations locales.

La question du rôle des aménités et de la qualité des lieux sur le développement économique est marquée par le décalage entre, d'un côté, une relation de causalité difficile à démontrer empiriquement et, d'un autre côté, des décideurs qui, dans le contexte de l'économie de la connaissance, soulignent l'importance croissante de ce type de ressources territoriales. En effet, « qu'ils soient de droite, de gauche, écologistes ou non, la plupart des décideurs publics s'entendent sur le fait que, si elles veulent attirer chez elles des entreprises de haute technologie et une main-d'œuvre hautement qualifiée, les villes du savoir ne peuvent plus se permettre d'axer leur stratégie de développement économique uniquement sur la réduction des coûts de production des entreprises ou sur les crédits d'impôt. Dorénavant, les différents acteurs urbains devront promouvoir les avantages socio-culturels, naturels ou encore la qualité de vie offerte par leur ville. Paradoxalement, très peu de chercheurs ont démontré empiriquement que l'attraction et la rétention de "talents" dans les villes du savoir reposaient sur la "bonne" qualité de vie offerte par ces dernières $"{ }^{67}$.

La recherche des aménités n'est pas qu'une question de localisation résidentielle. Elle concerne aussi les entreprises, en particulier les entreprises des domaines scientifiques et technologiques, qui sont généralement à la recherche d'une localisation de qualité. En Belgique, c'est pour ces profils d'entreprises que les parcs scientifiques ont été implantés dans des cadres naturels attractifs situés à proximité des grandes villes et des centres de recherche ${ }^{68}$. Aujourd'hui, la volonté de renforcer les centres urbains et de limiter la périurbanisation conduit à implanter les entreprises des domaines scientifiques et technologiques dans des localisations plus centrales, comme l'illustre le projet du Val Benoît à Liège ou la reconversion de l'Île de Nantes ${ }^{69}$. Ce type d'opérations est toutefois difficile à concrétiser en raison de la concurrence des parcs d'activités périphériques et des surcoûts imposés par le recyclage de friches urbaines ou industrielles ${ }^{70}$.

67 D. Naud, R. Tremblay, « Discours sur la qualité de vie et la compétitivité des villes du savoir », in D.-G. Tremblay, R. Tremblay, La compétitivité urbaine à l'heure de la nouvelle économie, op. cit., p. 57.

68 B. MÉRENNE-SCHOUMAKER, La localisation des industries. Enjeux et dynamiques, Rennes, Presses universitaires de Rennes, 2011.

69 V. TOURA, «De la décroissance urbaine aux processus d'aménagement participatifs. Étude de deux projets de renouvellement urbain en France : l'Île-de-Nantes et les Docks-de-Seine à Saint-Ouen ", Doctorales ASRDLF, Grenoble, 2019.

70 J.-M. Halleux, J.-M. LAmbotTe, « Reconstruire la ville sur la ville. Le recyclage morphologique et le renouvellement des espaces dégradés", Territoire(s) wallon(s), $\mathrm{n}^{\circ} 2,2008, \mathrm{p} .7-22$; P.-F. WILMOTTE, C. Ruelle, B. BianCHET, « Les enjeux de l'investissement privé en milieu urbain », op. cit. 
Au final, le sujet des aménités pose la question des stratégies de développement que les villes souhaitent mener afin d'offrir un ensemble de biens, d'avantages et de services tels qu'ils stimulent l'attractivité urbaine. Cette problématique de marketing urbain s'applique tant à la population locale tentée par l'exode urbain qu'à de potentiels nouveaux habitants, touristes ou investisseurs du secteur productif.

\subsubsection{Les facteurs matériels directement utilisés par les entreprises : les infrastructures de transport et le foncier à vocation économique}

À ce stade de la discussion, nous avons principalement traité de ressources territoriales immatérielles, à l'image du capital humain ou de la capacité d'innovation. Afin de compléter l'analyse, il est utile de poser la question du rôle sur le développement de facteurs matériels susceptibles d'être directement utilisés par les entreprises, à l'image des infrastructures de transport ou du foncier à vocation économique.

Vis-à-vis de cette question, un premier constat est le décalage entre, d'une part, la mise en exergue de l'enjeu des parcs d'activités par les acteurs de terrain et, d'autre part, l'intérêt limité que ce sujet rencontre auprès des chercheurs ${ }^{71}$. En effet, à la différence des élus, des entreprises et des agences de développement économique, les scientifiques actifs dans le champ des sciences régionales ne convoquent que très rarement l'impact de la disponibilité en foncier sur les niveaux de développement. Plutôt que l'enjeu des parcs d'activités, les chercheurs mettent principalement en exergue l'importance de facteurs immatériels tels que l'innovation, l'esprit d'entreprise, la formation, l'insertion dans les réseaux, etc. Ce décalage entre la perception des chercheurs et celle des opérateurs de terrain nous semble lié au fait que la politique des parcs d'activités est plus opportune en termes d'aménagement du territoire et de limitation des nuisances sur les voisinages qu'en termes de développement régional.

Afin d'approfondir le sujet des facteurs matériels, appuyons-nous sur une analyse récente dédiée à l'impact de la disponibilité foncière en parcs d'activités sur le développement économique des régions de l'Europe du Nord-Ouest ${ }^{72}$. Il ressort de cette analyse que les relations entre les disponibilités en terrains et les niveaux de développement varient en fonction des types de régions. Dans les régions métropolitaines, où les économies d'agglomération sont puissantes, la disponibilité foncière ne joue qu'un rôle marginal sur les niveaux de développement. Cela résulte de la présence de nombreuses activités à la fois peu extensives spatialement et très productives en valeurs ajoutées. La situation des régions métropolitaines s'oppose à la situation des régions intermédiaires. Dans les régions intermédiaires, la mise à disposition de terrains industriels et commerciaux a un réel impact sur le développement. Pour autant, les résultats quantitatifs indiquent que l'impact effectif demeure limité. On peut penser des régions intermédiaires que leur profil est adapté à des implantations d'activités qui, à l'image de la logistique, génèrent un volume limité de valeurs ajoutées par unité de surface. Pour les régions rurales, les modèles chiffrés

71 M.-C. VANDERMEER, J.-M. HALLEUX, « Evaluation of the spatial and economic effectiveness of industrial land policies in northwest Europe », European Planning Studies, volume 25, n 8, 2017, p. 1454-1575.

72 Ibidem, p. 1454-1575. 
indiquent que la disponibilité en terrains est corrélée de manière négative aux niveaux de développement. Il s'agit là d'un résultat contre-intuitif que nous interprétons par le fait que les opérateurs économiques de ces régions offrent d'autant plus de terrains que leur région est en retard de développement. Sans conteste, cela témoigne de politiques foncières inefficaces, qui amplifient l'étalement urbain et qui nécessiteraient donc d'être réajustées. En réalité, ces politiques d'offre foncière négligent le fait que la disponibilité en terrains est une condition certes nécessaire, mais certainement pas suffisante à la croissance économique et à la création d'emplois.

Le caractère nécessaire mais insuffisant semble également devoir s'appliquer à la ressource territoriale des infrastructures de transport. D'un côté, face aux continentalisation et mondialisation de l'économie, il faut être connecté aux marchés pour développer son économie productive. Par contre, de l'autre côté, les retombées liées à une amélioration de l'accessibilité pour les régions centrales et déjà dotées de bonnes connexions aux réseaux de transports sont globalement faibles pour les régions européennes ${ }^{73}$. Mobiliser la notion de l'avantage différenciatif et en constater l'absence permet de comprendre ce constat. En effet, au niveau des régions centrales du continent, la concurrence pour attirer les investissements se joue entre des territoires qui ne peuvent se différencier significativement vis-à-vis de l'accessibilité aux marchés. Cette situation concerne en particulier la Wallonie, qui est en concurrence avec de nombreuses régions (Flandre et régions étrangères) qui bénéficient des mêmes niveaux globaux d'accessibilité. Face à ce constat, il semble illusoire d'attendre que l'accessibilité de la Wallonie contribue significativement à son redéploiement productif.

En Wallonie comme ailleurs, des politiques publiques très onéreuses sont menées pour intervenir sur les facteurs matériels que nous venons de commenter. Alors que leur impact réel n'est pas scientifiquement avéré, l'explication à la mobilisation de moyens importants sans support scientifique réside vraisemblablement dans les demandes d'acteurs de terrain qui ont besoin d'être rassurés par des mesures tangibles et concrètes. Les « routes pour l'emploi » sont un exemple de politique wallonne qui mobilise des moyens financiers importants pour améliorer l'accessibilité routière aux parcs d'activités économiques (cf. infra). L'hypothèse sous-jacente à cette mesure est que l'accessibilité est un déterminant important quant à la capacité des entreprises à développer leurs activités. Pourtant, nous sommes ici face à une littérature scientifique à la fois peu développée et peu convergente ${ }^{74}$.

3 K. SPIEKERMANN et al., TRACC Transport Accessibility at regional/local scale and patterns in Europe, Luxembourg, ESPON, 2015.

J.-M. OfFNER, «Les effets structurants du transport : mythe politique, mystification scientifique », L'Espace géographique, volume 22, n³ 3, 1993, p. 233-242. 


\section{LES POLITIQUES PUBLIQUES EN MATIÈRE DE DÉVELOPPEMENT ÉCONOMIQUE ET TERRITORIAL}

Dans le premier chapitre, nous avons présenté un substrat théorique constitué de grilles de lecture sur les liens entre territoire et développement économique. L'objectif de ce deuxième chapitre est de fournir un substrat complémentaire sur les principales politiques publiques dédiées au développement économique et territorial de la Wallonie. Pour rappel, la politique du développement territorial renvoie aux diverses actions des autorités publiques en vue d'améliorer la performance des territoires vis-à-vis d'ambitions collectives de développement. Pour rappel également, ces ambitions collectives peuvent être économiques, ce qui nous intéresse directement dans cette publication, mais elles doivent également ressortir des enjeux de la soutenabilité environnementale et de la cohésion sociale.

Dans le champ du développement économique et territorial, les différentes réformes de l'État ont progressivement fait des Régions les principales autorités compétentes. Il ne faut toutefois pas négliger l'impact des initiatives de l'Union européenne, ni le rôle que le niveau fédéral continue d'assurer dans certains domaines conjoints.

Notre description des politiques territoriales qui ont un impact sur le développement économique de la Wallonie s'organise en deux sections. La première section traite du rôle joué par l'Union européenne. Elle permet d'analyser comment les principes de la politique régionale européenne s'appliquent à la Wallonie. La deuxième section est dédiée aux principaux programmes des autorités wallonnes et fédérales qui conjuguent une importante composante territoriale à l'objectif du renforcement de l'économie.

À l'instar du chapitre précédent, l'objectif principal de ce deuxième chapitre est, via une présentation relativement succincte, de préparer le lecteur aux analyses économiques et territoriales développées dans la suite de la publication. Pour le lecteur qui souhaiterait approfondir la thématique des politiques publiques menées en matière de développement territorial, il existe des publications spécialisées auxquelles il pourra accéder en s'appuyant sur les références bibliographiques que nous mentionnons.

\subsection{LE RÔLE DE L’UNION EUROPÉENNE}

Le rôle de l'Union européenne (UE) sur le développement économique et territorial de la Wallonie se joue à deux niveaux principaux. 
Le premier niveau est celui des grandes orientations stratégiques que l'UE décline pour les territoires de ses États membres. Il s'agit là du premier aspect que nous allons aborder dans cette section. Nous y commenterons la stratégie Europe 2020, ainsi que les stratégies de spécialisation intelligente ( smart specialisation strategy» ou S3).

Le second niveau est celui de la politique régionale européenne, aussi appelée la politique de cohésion. Avec la politique de cohésion, l'intervention de l'UE n'est plus seulement stratégique, mais également opérationnelle, avec en particulier l'usage des instruments financiers que sont les fonds structurels. Ces fonds correspondent à des aides non remboursables qui assurent, en complément des moyens mobilisés par les États membres, le cofinancement d'actions en faveur d'un développement territorial équilibré et durable.

L'objectif clé de la politique régionale européenne est de réduire les disparités sociospatiales entre les régions et pays de l'UE. Cet objectif fondamental a été mis en exergue dès le Traité de Rome, sur la base du constat qu'une union entre différents pays n'est possible à long terme que si les disparités socio-spatiales tendent à s'atténuer. C'est depuis 1989 que la politique régionale européenne repose sur le dispositif des fonds structurels. Depuis lors, elle a été mise en œuvre lors de cinq périodes de programmation : 1989-1993, 1994-1999, 2000-2006, 2007-2013 et 2014-2020. Dans la suite de ce chapitre, nous nous intéresserons principalement à l'actuelle programmation ${ }^{75}$.

Une approche en deux temps sera suivie pour décrire les aspects économiques et territoriaux de la politique régionale européenne en Wallonie. Tout d'abord, nous décrirons les lignes directrices de la stratégie wallonne vis-à-vis de la programmation 2014-2020. Ensuite, nous affinerons l'analyse en présentant les choix infra-régionaux du gouvernement wallon et la géographie prioritaire des investissements. Compte tenu du sujet de la publication, notre focus sur la politique régionale européenne est limité au champ couvert par le Fonds européen de développement régional (FEDER). Par contre, nous ne développons pas le champ couvert par le Fonds social européen (FSE). Bien que ce fonds mobilise des moyens importants et qu'il finance des projets relatifs à la formation, ses composantes territoriales sont peu affirmées par rapport à celles du FEDER. À l'échelle wallonne, les moyens mobilisés par le Programme opérationnel FEDER 2014-2020 sont très conséquents. Ils représentent un budget global de 1,703 milliard d'euros, dont 681 millions sont financés directement par l'UE.

La prise en compte de l'influence des politiques européennes sur le développement territorial en Wallonie aurait pu intégrer d'autres dimensions, par exemple les politiques menées en matière d'agriculture (la Politique agricole commune - PAC) ou en matière d'investissements en infrastructures de transport. Nous n'avons toutefois pas jugé opportun de développer ces sujets vu leurs relations finalement assez ténues vis-à-vis de l'enjeu du redéploiement de l'économie wallonne. S'agissant de la question agricole, force est de constater que, au regard des indicateurs d'emplois et de valeurs ajoutées, les activités rurales s'avèrent assez peu structurantes. Concernant la thématique des infrastructures de transport, rappelons ici qu'une des conclusions de notre état de l'art est que l'impact effectif de l'amélioration des accessibilités sur la croissance s'apparente

75 Les lecteurs intéressés pourront approfondir la thématique avec des publications spécialisées sur l'historique de la politique régionale en Belgique et en Europe. Cf., par exemple, H. CAPrON, "Croissance et développement spatial inégal des régions ", in B. BAYENET, H. CAPRON, P. LIÉGEOIS (dir.), L'espace WallonieBruxelles. Voyage au bout de la Belgique, Bruxelles, De Boeck, 2007, p. 199-226. 
davantage à un mythe qu'à une réalité scientifiquement objectivée (cf. supra, 1.2.5) - en tout cas pour les régions déjà bien connectées.

\subsubsection{Les orientations stratégiques de l'Union européenne}

L'UE aspire à accroître la compétitivité économique de l'ensemble de ses États membres. C'est dans cette perspective que la stratégie Europe 2020 a été adoptée en 2010. Cette stratégie vise à coordonner les politiques économiques au sein de l'UE sur la période 2010-2020. Elle est associée à huit indicateurs principaux, dont quatre concernent directement les enjeux du développement économique, des marchés du travail et de l'éducation ${ }^{76}$. Les quatre indicateurs en question sont les suivantes: le taux d'emploi, la part du PIB investie dans le secteur de la R\&D, la part de diplômés de l'enseignement supérieur chez les 30-35 ans et le taux d'abandon scolaire précoce (ASP) ${ }^{77}$. Au chapitre 3 , nous aurons l'occasion d'analyser comment la Wallonie et ses provinces se positionnent vis-à-vis de ces indicateurs.

Compte tenu des disparités socio-spatiales et des stratégies nationales au sein de l'UE, les objectifs relatifs aux quatre indicateurs varient en fonction des États membres, comme l'illustre le tableau 2, qui recense les objectifs en question pour l'ensemble de l'UE ainsi que pour la Belgique et ses pays limitrophes ${ }^{78}$.

Tableau 2. Objectifs nationaux de la stratégie Europe 2020 pour les quatre indicateurs relatifs au développement territorial

\begin{tabular}{|l|c|c|c|c|}
\cline { 2 - 4 } \multicolumn{1}{c|}{} & Taux d'emploi & $\begin{array}{c}\text { Part du PIB investie } \\
\text { dans la R\&D }\end{array}$ & $\begin{array}{c}\text { Part de diplômés } \\
\text { de l'enseignement } \\
\text { supérieur } \\
\text { chez les 30-35 ans }\end{array}$ & $\begin{array}{c}\text { Taux d'abandon } \\
\text { scolaire précoce }\end{array}$ \\
\hline Allemagne & $77,0 \%$ & $3,0 \%$ & $42,0 \%$ & $10,0 \%$ \\
Belgique & $73,2 \%$ & $3,0 \%$ & $47,0 \%$ & $9,5 \%$ \\
France & $75,0 \%$ & $3,0 \%$ & $50,0 \%$ & $9,5 \%$ \\
Luxembourg & $73,0 \%$ & $2,5 \%$ & $66,0 \%$ & $10,0 \%$ \\
Pays-Bas & $80,0 \%$ & $2,5 \%$ & $40,0 \%$ & $8,0 \%$ \\
\hline Union européenne & $75,0 \%$ & $3,0 \%$ & $40,0 \%$ & $10,0 \%$ \\
\hline
\end{tabular}

Sources : Eurostat, «Europe 2020. Overview», https://ec.europa.eu ; Eurostat, «Europe 2020 Targets », 7 mars 2017, https://ec.europa.eu.

En matière d'orientations stratégiques, l'UE joue également un rôle dans le cadre de la politique industrielle. Depuis les années 2000, la Commission européenne alimente une réflexion sur la compétitivité de l'industrie européenne. Cette réflexion est formalisée via des stratégies de spécialisation intelligente, dont l'objectif est d'aider les acteurs des régions européennes à définir et/ou à adapter leurs propres politiques économiques et industrielles.

76 Quatre autres objectifs ont été définis dans les domaines de la lutte contre la pauvreté, de l'énergie et de l'environnement. Ils ne sont pas traités directement ici.

77 Le taux d'abandon scolaire précoce (ASP) est mesuré par la proportion des jeunes entre 18 et 24 ans qui ont quitté l'école en n'ayant achevé que l'enseignement secondaire inférieur (ou moins) et qui ne poursuivent ni étude, ni formation.

78 Des objectifs nationaux n’ont pas été définis pour le Royaume-Uni, ceci indépendamment de la question du Brexit. 
La philosophie des spécialisations intelligentes s'inscrit dans la logique des avantages différenciatifs décrite ci-dessus. L'objectif des spécialisations intelligentes est que les régions européennes s'appuient sur les acteurs économiques des territoires pour développer des systèmes productifs basés sur des niches spécifiques de biens et de services. Sous le pilotage du gouvernement wallon, l'administration régionale effectue depuis plusieurs années un travail de réflexion afin de développer une stratégie de spécialisation intelligente pour la Wallonie ${ }^{79}$. Cela nécessite une coordination accrue tant entre le niveau européen et le niveau wallon qu'entre les différents programmes mis en ouvre par les autorités de la Wallonie, à l'image du Plan Marshall et du programme Creative Wallonia (cf. infra).

\subsubsection{Les lignes directrices de la stratégie wallonne vis-à-vis de la programmation 2014-2020 de la politique régionale}

La programmation actuelle de la politique régionale européenne couvre la période 20142020. Cette programmation se concentre sur la poursuite des objectifs de la stratégie Europe 2020, dans la perspective économique et industrielle des spécialisations intelligentes («smart specialisations») que nous venons d'invoquer.

Pour la programmation 2014-2020, la Commission européenne a défini des balises à respecter par les autorités des États membres ${ }^{80}$. Dans le cadre de l'intervention du FEDER en Wallonie, les balises principales à considérer et à respecter sont les suivantes :

- dix thèmes prioritaires ont été définis par la Commission européenne et la Wallonie doit inscrire ses investissements dans ces dix thèmes ;

- une cartographie des interventions de la politique régionale a été définie par la Commission européenne à l'échelle des NUTS $2^{81}$, plaçant la province de Brabant wallon parmi les régions les plus développées tandis que les quatre autres provinces wallonnes sont reprises comme des "régions en transition », correspondant aux NUTS 2 dont le PIB par habitant est situé entre $75 \%$ et $90 \%$ de la moyenne de l'UE ;

- une grande majorité des investissements doit s'effectuer dans des thèmes à vocation économique ou liés à la transition vers une économie plus sobre en carbone.

C'est sur la base de ces balises qu'ont eu lieu les négociations entre la Commission européenne et les Régions belges pour la répartition des moyens entre les entités fédérées. L'Autorité fédérale n'y a joué qu'un rôle de compilation et d'ensemblier car l'essentiel des moyens européens sont alloués aux entités fédérées. C'est également sur cette base que, pour le FEDER en particulier, le gouvernement wallon a préparé un programme

79 «Stratégie de spécialisation intelligente de la Wallonie. Vers une politique régionale d'innovation industrielle durable », Namur, SPW, 2015.

80 Ces balises ont été fixées dans le cadre du règlement (UE) nº 1301/2013 du Parlement européen et du Conseil du 17 décembre 2013 relatif au Fonds européen de développement régional et aux dispositions particulières relatives à l'objectif « Investissement pour la croissance et l'emploi », et abrogeant le règlement (CE) $n^{\circ}$ 1080/2006 [du Parlement européen et du Conseil du 5 juillet 2006 relatif au Fonds européen de développement régional et abrogeant le règlement (CE) $n^{\circ} 1783 / 1999$ ] (Journal officiel de l'Union européenne, L347, 20 décembre 2013).

${ }^{81}$ Nomenclature des unités territoriales statistiques, permettant le découpage par Eurostat, l'autorité statistique européenne, des pays européens en entités comparables. Le niveau NUTS 2 correspond, par exemple, aux provinces belges et aux régions françaises avant l'entrée en vigueur du nouveau découpage régional au $1^{\mathrm{er}}$ janvier 2016. 
opérationnel visant à fixer ses propres orientations thématiques et géographiques ${ }^{82}$. En complément à la préparation du programme opérationnel, l'autorité wallonne a mis en place une task force composée d'experts du monde universitaire, de représentants des entreprises et de gestionnaires du Plan Marshall. Cette task force avait pour mission d'opérer la sélection des projets à subventionner.

Les orientations définies par le gouvernement wallon s'inscrivent dans des axes prioritaires qui ont pris place à l'intérieur des dix thèmes prioritaires définis par la Commission européenne. Il ressort de l'analyse du programme opérationnel wallon que ces axes privilégient deux catégories d'enjeux : d'une part, les enjeux du développement urbain (principalement à travers les axes « Développement urbain 2020 », «Transition vers une Wallonie bas carbone » et «Intelligence territoriale 2020 ») et, d'autre part, les enjeux de la compétitivité économique (principalement à travers les axes «Innovation 2020 ", «Économie 2020 » et «Compétence 2020 »). Cette seconde catégorie d'enjeux est associée à des aides à l'investissement destinées aux entreprises.

La focalisation des autorités wallonnes sur les enjeux de la compétitivité économique et du développement urbain n'est guère étonnante. Elle s'explique, d'une part, parce que les principaux décideurs wallons perçoivent le redéploiement de l'économie comme une nécessité vitale et, d'autre part, parce qu'il est de plus en plus évident que les villes, et les grandes villes en particulier, sont devenues les vecteurs privilégiés de la croissance économique. En parallèle, il est manifeste que la mise en œuvre de la politique régionale européenne par les autorités wallonnes s'inscrit clairement dans le paradigme du développement territorial. Le programme opérationnel wallon l'illustre avec ses très nombreuses références aux économies d'agglomération et à la métropolisation.

Globalement, le programme opérationnel préparé par les autorités wallonnes vise à ce que les moyens mobilisés par le FEDER permettent de dynamiser les ressources territoriales associées au(x) territoire(s) wallon(s). Les aides aux entreprises visent à stimuler leur capacité d'innovation pendant que les moyens du FSE sont mobilisés à destination des centres de formation afin de renforcer les compétences et la créativité des travailleurs. Des investissements sont consacrés au milieu urbain afin d'améliorer les aménités dans une perspective de développement économique. Enfin, les facteurs matériels sont loin d'être négligés, avec des investissements substantiels dédiés à l'aménagement des parcs d'activités et à l'amélioration de leur accessibilité. À la lumière de la littérature scientifique exposée précédemment (cf. supra, 1.2.5), ce dernier point relatif aux facteurs matériels apparaît comme le plus discutable.

\subsubsection{Les choix infra-régionaux du gouvernement wallon et la géographie prioritaire des investissements}

Sur la base des balises définies par l'UE (choix des provinces prioritaires, focalisation sur certaines priorités socio-économiques, transversalité des investissements, etc.), le gouvernement wallon a arrêté une géographie prioritaire pour les aides destinées aux projets de développement urbain et au renforcement de la compétitivité des entreprises.

82 Gouvernement wallon, «Wallonie-2020.EU. La stratégie wallonne pour une croissance intelligente, durable et inclusive en partenariat avec l'Union européenne. Programme opérationnel FEDER (Version 4.0)», Namur, SPW, 2018. 


\subsubsection{Les aides destinées aux projets de développement urbain}

Rappelons que les aides destinées aux projets de développement urbain s'inscrivent principalement dans trois axes du Programme opérationnel FEDER : "Développement urbain 2020 », « Transition vers une Wallonie bas carbone » et « Intelligence territoriale $2020 »$.

Les principaux objectifs de l'axe « Développement urbain intégré 2020 » sont de favoriser l'attractivité urbaine, mais également de dynamiser l'emploi dans les zones urbaines et de contribuer à la réduction des émissions de gaz à effet de serre. Pour cet axe, les projets sélectionnés devaient être situés au sein de douze autorités locales appartenant à quatre types de territoires. Il s'agit, premièrement, de Charleroi et de Liège, les deux métropoles potentielles que compte la Wallonie. En effet, ces deux villes sont les plus importantes de Wallonie et les seules qui, à relativement court terme, pourraient prétendre au statut de métropole européenne ${ }^{83}$. Il s'agit, deuxièmement, de sept villes moyennes ayant été fortement affectées par le déclin industriel : Herstal, La Louvière, Mons, Sambreville, Seraing et Verviers. Il s'agit, troisièmement, de trois villes moyennes proches de pôles métropolitains étrangers: Arlon, Mouscron et Tournai. Pour ces trois villes, les dynamiques transfrontalières sont particulièrement stratégiques. Il s'agit, quatrièmement, de Namur, au titre de capitale régionale.

Concernant l'axe «Transition vers une Wallonie bas carbone », l'ensemble du territoire régional est éligible pour les aides aux entreprises, mais seules les douze autorités locales éligibles pour l’axe « Développement urbain intégré 2020 » demeurent éligibles pour les aides aux acteurs urbains.

L'axe «Intelligence territoriale 2020 » vise à respecter une balise définie par la politique régionale européenne afin que les Régions investissent une partie des montants disponibles dans des projets urbains intégrés. Il soutient des projets au sein des douze autorités locales citées, mais également des projets situés dans les périphéries des pôles en question. Pour cet axe, l'attribution des moyens disponibles s'est basée sur une négociation entre, d'une part, le gouvernement régional appuyé par la task force chargée de la sélection des projets et, d'autre part, les représentants des douze villes et des entités chargées de l'élaboration et de la concrétisation des stratégies infra-régionales ${ }^{84}$.

\subsubsection{La compétitivité économique et les aides à l'investissement destinées aux entreprises}

Les aides destinées aux entreprises s'inscrivent dans les axes "Économie 2020 ", « Innovation 2020 » et " Transition vers une Wallonie bas carbone ». Elles s'adressent à toutes les entreprises reprises dans les secteurs économiques stimulant la base économique régionale, à savoir les secteurs des clusters et des pôles de compétitivité, le tourisme, les services aux entreprises et la R\&D. Le montant des aides dépend à la fois de la taille des entreprises et de leur localisation.

83 J.-F. Thisse, I. THOMAS, « Bruxelles et Wallonie », op. cit., p. 75-93.

Ces entités infra-régionales correspondent au GRE en province de Liège, à l'AXUD pour Namur et Sambreville, au Comité de développement stratégique de la région de Charleroi Sud-Hainaut pour Charleroi, à RéseauLux pour Arlon, au Conseil de développement de Wallonie picarde pour Tournai et Mouscron et au Partenariat stratégique local « Cœur de Hainaut, Centre des énergies » pour La Louvière et Mons. 
La taille des entreprises est appréhendée selon une typologie en trois catégories : les grandes entreprises (GE), qui totalisent plus de 250 emplois en équivalent temps plein (ETP), les entreprises moyennes (EM), qui comptent de 50 à $250 \mathrm{ETP}$, et les petites entreprises (PE), qui comptent moins de $50 \mathrm{ETP}$. La localisation des entreprises est également appréhendée selon trois catégories : les communes de la zone 1, les communes de la zone 2 et les communes de la zone 3 (cf. Carte 1).

Pour la zone 2, les aides à l'investissement peuvent représenter jusque $30 \%$ pour les $\mathrm{PE}$, $20 \%$ pour les EM et $10 \%$ pour les GE. Jusque 2018, ces trois taux étaient majorés de $5 \%$ pour les entreprises situées en zone 1 . Pour la zone 3, seules les PE et les EM sont soutenues dans leur effort d'investissement, avec des taux respectifs d'intervention de $20 \%$ et de $10 \%$.

Carte 1. Catégories de communes prises en compte pour les aides à l'investissement destinées aux entreprises selon le Programme opérationnel FEDER 2014-2020

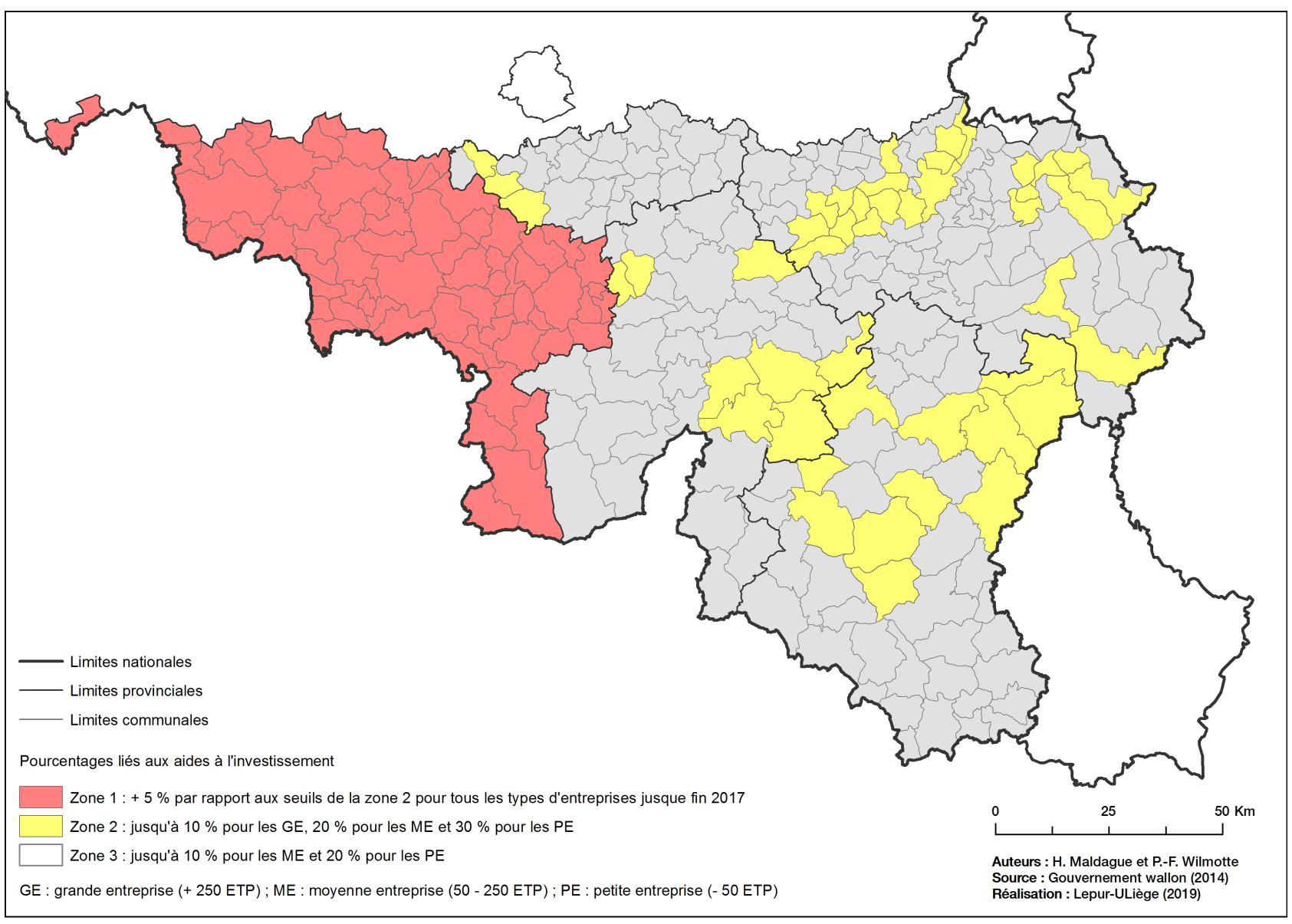


La zone 1, la plus avantagée, correspond au Hainaut. Cette province a bénéficié d'un régime avantageux du fait de son ancien statut de région prioritaire lors des précédentes programmations de la politique régionale ${ }^{85}$. La délimitation de la zone 2 est également héritée des géographies prioritaires des précédentes programmations, en tout cas partiellement. C'est ainsi que l'on trouve en zone 2 les communes des bassins industriels liégeois et verviétois déjà soutenues par les anciens programmes «FEDER Objectif 2 » relatifs aux régions en déclin industriel. On trouve également en zone 2 les petites villes et communes rurales de l'Ardenne précédemment soutenues par l'ancien programme « FEDER Objectif 5 » relatif au développement rural.

D'autres communes ont été ajoutées à la zone 2, cela pour deux raisons. Une première raison est la proximité du Hainaut, et donc sa concurrence sur les investissements potentiels. Cela a justifié d'y intégrer les communes suivantes : Ittre, Jemeppe-sur-Sambre, Nivelles, Sambreville et Tubize. La géographie de la zone 2 est également liée à la volonté de créer des ensembles continus qui représentent au moins 100000 habitants, conformément à la réglementation européenne cherchant à focaliser les moyens sur des ensembles cohérents. L'analyse de la manière dont les décideurs wallons ont appliqué cette réglementation européenne montre qu'ils ont tenu compte de la géographie des parcs d'activités. Cela permet de comprendre pourquoi le bassin économique liégeois a été étendu à l'Ouest vers les parcs d'activités créés le long de la E42 (Andenne, Villers-leBouillet) ou vers les communes en reconversion de la vallée mosane (Amay). Cela permet également de comprendre pourquoi les communes de Baelen, d'Eupen, de Lontzen, de Thimister-Clermont et de Welkenraedt ont été ajoutées au bassin verviétois. En effet, ce sont ces communes qui hébergent les principaux parcs d'activités de l'est de la province de Liège.

L'intégration à la zone 2 de communes où l'on trouve d'importants parcs d'activités fait sens puisque les principaux bénéficiaires potentiels des aides à l'investissement sont situés au sein de parcs. Afin de l'expliquer, il nous semble également opportun de revenir sur le fait que, en Wallonie, l'outil du parc d'activités demeure le symbole de la politique du développement régional. Il apparaît toutefois légitime de s'interroger sur l'impact effectif d'une politique qui vise à offrir du foncier bon marché aux entreprises (cf. supra, 1.2.5).

Pour sa part, la zone 3 rassemble les communes qui n'appartiennent ni à la zone $1 \mathrm{ni}$ à la zone 2. Comme nous venons de le mentionner, les entreprises situées en zone 3 bénéficient des aides à l'investissement les plus réduites. En complément de la carte 1, le tableau 3 rend compte des principaux choix effectués par les autorités européennes et régionales pour les échelles provinciale, supracommunale et communale.

85 En référence aux programmes « Objectif 1 » pour les territoires en grand retard de développement de la politique régionale européenne qui a concerné la province de Hainaut durant les décennies 1990 et 2000. 
Tableau 3. Principaux choix spatiaux opérés par les autorités européennes et régionales pour la programmation 2014-2020 de la politique de cohésion

\begin{tabular}{|c|c|}
\hline & Principales discriminations territoriales \\
\hline Échelle provinciale & $\begin{array}{l}\text { Focalisation des moyens sur les provinces de Hainaut, de Liège, de Namur } \\
\text { et de Luxembourg. Les entreprises de la province de Hainaut bénéficient } \\
\text { d'une intervention plus favorable (zone 1). }\end{array}$ \\
\hline Échelle supra-communale & $\begin{array}{l}\text { Outre la province de Hainaut, les bassins industriels de Liège et d'Eupen- } \\
\text { Verviers ainsi que les communes ardennaises (axe Dinant-Saint-Vith) } \\
\text { bénéficient aussi d'une aide majorée sur les investissements (zone 2). } \\
\text { Les sociétés publiques d'investissement infra-régionales bénéficient } \\
\text { de moyens spécifiques pour investir et soutenir les entreprises locales. } \\
\text { Concernant le développement urbain, les territoires d'intervention } \\
\text { privilégiés sont : les pôles urbains majeurs de Charleroi et de Liège, } \\
\text { la capitale régionale Namur, les villes moyennes en déclin industriel } \\
\text { et les villes moyennes des aires frontalières. }\end{array}$ \\
\hline Échelle communale & $\begin{array}{l}\text { Douze villes sont éligibles à l'axe " développement urbain intégré », } \\
\text { sous l'égide d'institutions infra-régionales censées aider les villes retenues } \\
\text { dans la planification stratégique et le montage des projets : Charleroi } \\
\text { et Liège comme grandes villes; Herstal, La Louvière, Mons, Sambreville, } \\
\text { Seraing et Verviers compte tenu de leur déclin industriel; Arlon, Mouscron } \\
\text { et Tournai pour leur positionnement frontalier; Namur comme capitale } \\
\text { régionale. }\end{array}$ \\
\hline
\end{tabular}

Source : synthèse établie à partir de Gouvernement wallon, « Wallonie-2020.EU. La stratégie wallonne pour une croissance intelligente, durable et inclusive en partenariat avec l'Union européenne. Programme opérationnel FEDER. Version 6.0 (version approuvée par la Commission le 30 janvier 2020 Décision C(2020) 590) », http://europe.wallonie.be.

\subsection{LES POLITIQUES ÉCONOMIQUES}

Après avoir présenté la manière dont la politique régionale européenne se décline en Wallonie, nous allons compléter notre panorama des politiques publiques par la prise en compte des principaux programmes qui ont un impact sur le développement économique de cette région. Pour ce faire, nous allons débuter par l'emblématique Plan Marshall, avant de poursuivre par les politiques de créativité et du numérique de l'autorité régionale wallonne. Nous terminerons avec l'initiative fédérale relative aux zones franches.

\subsubsection{Le Plan Marshall 4.0}

Initiés au milieu des années 2000, différents plans, dits Marshall, se sont succédé depuis lors. Notre objectif n'est pas de présenter l'historique de ces différents plans, ni de les analyser en détail ${ }^{86}$. Par contre, nous souhaitons permettre au lecteur de comprendre comment ces plans articulent les questions du développement économique et du développement territorial. Pour ce faire, nous allons passer en revue les axes prioritaires

86 À ce propos, cf. A. ACCAPUto, B. BAYenet, G. PAGAnO, «Le Plan Marshall pour la Wallonie », op. cit.; C. DUJARDIN et al., "Les pôles de compétitivité wallons», op. cit.; C. DUJARDIN et al., "L'évaluation des pôles de compétitivité wallons », op. cit. 
du Plan Marshall 4.0 ${ }^{87}$. Ce plan, qui représente un budget de 2,9 milliards d'euros sur la période 2015-2019, s'articule autour des axes suivants : la formation et l'orientation, l'innovation et la croissance, l'innovation numérique, le développement territorial, l'énergie et l'économie circulaire.

L'axe relatif à l'énergie et à l'économie circulaire représente un budget très conséquent de 1,1 milliard d'euros. Il vise à soutenir l'efficacité et la transition énergétique, avec des actions qui concernent la dimension environnementale plutôt que la dimension économique du développement territorial. À la différence des autres axes, il ne présente donc que des liens indirects et ténus avec le sujet du présent Courrier hebdomadaire.

L'axe relatif à la formation est intitulé « Faire du capital humain un atout ». Il représente un budget de 304,5 millions d'euros. Les moyens significatifs mobilisés via cet axe témoignent de l'enjeu crucial de la ressource territoriale du capital humain pour une région de tradition industrielle comme la Wallonie. Les disparités socio-spatiales en capital humain sont considérables en Wallonie. Afin de les limiter et afin d'améliorer la situation globale de la région, les moyens que mobilise le plan sont principalement alloués aux domaines suivants: l'alternance comme filière d'excellence (stages en entreprises, valorisation des compétences, etc.), le développement de l'orientation des apprenants, la connaissance des langues étrangères et la facilitation de l'accès à l'enseignement supérieur et à la formation tout au long de la vie.

Les actions du Plan Marshall 4.0 en matière d'innovation et de croissance représentent 850,5 millions d'euros. Elles s'inscrivent dans l'intitulé suivant: "Soutenir le développement de l'industrie par une politique d'innovation et de croissance des entreprises». Les moyens disponibles dans cet axe visent à soutenir les pôles de compétitivité et à aider les entreprises pour leur $\mathrm{R} \& \mathrm{D}$ et leur internationalisation. La philosophie sous-jacente à cet axe a été détaillée dans notre premier chapitre. Elle repose sur le constat que, dans le contexte de l'économie de la connaissance, l'innovation est devenue vitale pour les entreprises. En outre, cette philosophie repose aussi sur le postulat qu'il est possible de rendre un territoire plus attractif et plus compétitif en stimulant l'innovation des entreprises qu'il héberge. Comme nous l'avons également détaillé au chapitre précédent, les politiques en matière d'innovation s'articulent aux politiques de clustering, dont l'objectif est de stimuler l'innovation en stimulant les contacts entre entreprises ainsi qu'entre entreprises et centres de recherche.

La question de l'innovation dans le domaine du numérique fait l'objet d'un axe spécifique titré «Soutenir l'innovation numérique ». Il représente 244,8 millions d'euros et il vise à renforcer l'intégration du numérique dans la gestion publique, la mobilité et le développement urbain. Le territoire y est donc concerné à plusieurs titres : par le développement des smart cities, par la mise en ouvre de lignes de transport en commun à haut niveau de service, par le développement d'un réseau express de covoiturage, par le déploiement du très haut débit à travers la Wallonie et par le développement de lieux de travail innovants (fablabs, hubs créatifs, etc.).

L'axe relatif au développement territorial est intitulé «Mobilisation du territoire à destination du développement économique ». Il représente un budget de 374 millions d'euros. La conception du développement territorial à laquelle il renvoie est restreinte.

87 Cf. le site Internet http://planmarshall.wallonie.be. 
En effet, les projets auxquels il correspond se limitent principalement à ce que nous avons décrit au chapitre précédent comme les ressources liées aux facteurs matériels des infrastructures de transport et du foncier à vocation économique. En l'occurrence, l'axe «Développement territorial» du Plan Marshall 4.0 vise notamment l'aménagement de parcs d'activités et de zones portuaires ainsi que la création d'infrastructures pour améliorer l'accès routier aux pôles d'activités (via une mesure intitulée les « Routes pour l'emploi »). Comme nous l'avons relevé au premier chapitre, les moyens mobilisés sur ces facteurs matériels sont considérables, alors même que la littérature scientifique est peu convergente sur leur réelle efficacité dans des régions déjà bien équipées comme, en l'occurrence, la Wallonie.

\subsubsection{Les politiques de créativité et du numérique de l'autorité régionale wallonne}

En complément aux Plans Marshall successifs, les autorités wallonnes ont développé le programme Creative Wallonia et la stratégie Digital Wallonia. Ces initiatives, mises en place durant la décennie 2010, s'inscrivent dans le paradigme de l'économie créative et culturelle. Ce paradigme repose sur l'idée que la créativité, l'inventivité et les capacités en matière de numérique dans les différentes dimensions de la vie en société sont des facteurs qui renforcent la capacité d'innovation des entreprises sises sur un territoire.

Appliquer le paradigme de l'économie créative et culturelle vise à renforcer l'ancrage territorial des relations entre la créativité et l'innovation, par le développement d'un écosystème favorable d'infrastructures, d'acteurs et de mesures politiques rendant le territoire attractif auprès des porteurs de projets créatifs et des activités créatives des secteurs de l'art et de la culture. Le «hub créatif » est un outil clé des politiques en faveur de l'économie créative. Il s'agit d'un support à l'écosystème qui peut être défini de la manière suivante: "Des plateformes d'organisation centrées sur la transformation de l'économie traditionnelle en économie créative à travers la mise en capacité des acteurs en favorisant l'innovation ouverte, l'hybridation transdisciplinaire et l'intelligence collaborative ${ }^{88}$.

Les moyens mobilisés par Creative Wallonia ont permis le développement de hubs créatifs. En soutenant des projets culturels et la créativité de la population, ils ont également ciblé les ressources territoriales des aménités urbaines et du capital humain. Ces actions recoupent les principes de la théorie de la classe créative, bien qu'il s'agisse ici de développer les talents des populations locales plutôt que d'attirer des talents issus d'autres territoires. Pour Digital Wallonia, l'objectif est de renforcer le numérique en tant que compétence, en tant qu'activité économique et aussi comme source de simplification administrative.

La Wallonie n'a pas le monopole des politiques basées sur le paradigme de l'économie créative et culturelle. La préparation d'un Cultural and Creative Cities Monitor par la Commission européenne ${ }^{89}$ illustre ici la montée en puissance de ces politiques urbaines

88 «Des hubs créatifs pour booster l'économie créative et l'innovation en Wallonie », 26 juin 2017, http://planmarshall.wallonie.be.

9 V. Montalto, C. Jorge TaCaO Moura, S. LANGediJK, M. Saisana, The cultural and creative cities monitor (2017 edition), Luxembourg, Publication Office of European Union, 2017. 
en faveur de l'économie créative et culturelle. En l'occurrence, ce moniteur urbain a analysé la situation de 168 villes au sein de 30 pays européens. Cela a été réalisé sur la base de trois dimensions principales : l'importance des activités culturelles, le poids de l'économie créative et le caractère favorable de l'environnement urbain vis-à-vis des domaines de la créativité et de la culture ${ }^{90}$.

\subsubsection{Les zones franches proposées par l'Autorité fédérale}

En Belgique, l'échelon fédéral dispose toujours de leviers d'action importants pour la compétitivité économique des territoires, avec en particulier des compétences susceptibles d'avoir un impact sur le coût du travail, la fiscalité et les réglementations ${ }^{91}$. Ces leviers d'action influencent bien sûr la compétitivité internationale du pays, en particulier vis-à-vis des pays voisins. Pour autant, ces leviers sortent du cadre du présent Courrier hebdomadaire car, pour l'essentiel, ils s'appliquent de manière indifférenciée sur l'ensemble $\mathrm{du}$ territoire national. Une exception à cette règle correspond à la politique fiscale des zones franches, un dispositif de discrimination territoriale positive qui instaure des périmètres à l'intérieur desquels les coûts de main-d'œuvre sont réduits.

Le dispositif des zones franches s'inscrit généralement dans une perspective d'équité territoriale, avec des mesures réservées à des zones en difficultés économiques. La littérature consacrée à l'impact du dispositif ne permet pas de conclure quant à son efficacité. Plus généralement, la littérature sur le développement économique régional conduit à relativiser l'impact de mesures qui viseraient à « compenser » et à « corriger» les handicaps de compétitivité d'un territoire - à l'image du coût du travail en Belgique et en Wallonie. Par contre, la même littérature souligne l'intérêt de stimuler les opportunités de croissance par l'articulation d'une combinaison originale de ressources ancrées dans le territoire ${ }^{92}$. Nous retrouvons ici la perspective des avantages différenciatifs (cf. supra, 1.1.5).

S'il apparait que le principe des zones franches est remis en question par les travaux scientifiques récents, un tel dispositif a pourtant été proposé par l'Autorité fédérale aux différentes Régions. Cette proposition s'appuie sur la loi du 15 mai 2014 portant exécution du pacte de compétitivité, d'emploi et de relance. Selon cette législation, une petite ou moyenne entreprise (PME) jugée en «bonne santé financière » et située au sein d'une zone franche peut bénéficier d'un abattement fiscal de $25 \%$ du précompte professionnel sur tout nouvel emploi créé dans le cadre d'un investissement de l'entreprise. Si l'emploi est pérennisé, cet avantage fiscal est octroyé pour trois ans.

La définition des territoires concernés par les zones franches relève de la concertation entre l'Autorité fédérale et les autorités régionales. L'Autorité fédérale a défini des critères que les propositions des autorités régionales doivent rencontrer : les Régions peuvent

90 Le lecteur intéressé pourra compléter son information à partir de «The cultural and creative cities monitor: 2019 edition ", Commission européenne, EU Science hub, https://ec.europa.eu (cf. notamment " The cultural and creative cities monitor: 2019 edition. Belgium », https://publications.jrc.ec.europa.eu).

91 B. MÉRENNE-SCHOUMAKER, La localisation des industries, enjeux et dynamiques, op. cit.

92 O. Meunier, M. Mosty, Évaluation du Plan Marshall 2.Vert. Évaluation thématique $n^{\circ} 6$ : Soutien à l'investissement dans les zones franches urbaines et rurales, Namur, IWEPS, 2014 ; P. NIJKAMP, « The resourceful region », op. cit., p. 191-214. 
proposer jusqu'à quatre sites ayant connu un « licenciement collectif ${ }^{93}$ autour desquels des zones franches seront créées pour une durée limitée dans le temps, à savoir six ans pour la Wallonie $^{94}$.

Sur cette base, les autorités régionales wallonnes ont proposé de sélectionner quatre sites ayant connu un licenciement collectif récent : ArcelorMittal à Seraing, Doosan à Frameries, Caterpillar à Gosselies et Saint-Gobain Sekurit à Sambreville. Autour de ces sites, l'ensemble des espaces à vocation économique situés à moins de 40 kilomètres des entreprises citées ont été sélectionnés, à savoir les parcs d'activités économiques reconnus par la Région, les infrastructures d'accueil de l'activité économique, les zones d'activités économiques industrielles au plan de secteur contiguës aux parcs d'activités économiques existants, les terrains des ports autonomes, les zones d'activités économiques mixtes au plan de secteur hors commerce de détail et les zones d'extraction au plan de secteur. Ces zones sont activées depuis le $1^{\text {er }}$ novembre 2017. En Flandre, les autorités régionales ont mis en œuvre leurs zones franches autour des sites Ford à Genk et Philips à Turnhout. Elles ont été mises en œuvre dès 2015 et elles englobent l'essentiel des terrains et immeubles à vocation économique situés dans les provinces d'Anvers et de Limbourg.

La présentation de ce dispositif des zones franches renvoie à trois problématiques qui, sans conteste, mériteraient des investigations complémentaires. Primo, comme cela a déjà été évoqué, le dispositif en question mobilise des moyens publics considérables alors que son efficacité en termes de développement régional reste à démontrer. Secundo, force est de constater que les zones franches flamandes ont été effectives plus rapidement que les zones franches wallonnes. Il en résulte que des entreprises situées en Flandre ont profité des abattements fiscaux deux années avant qu'ils ne bénéficient à des entreprises situées en Wallonie. Tertio et enfin, le choix des autorités wallonnes de n'appliquer le dispositif que sur des terrains à vocation purement économique situés en marge des zones d'habitat peut être questionné. Cette optique pénalise les entreprises qui ont fait le choix d'une localisation dans les centres urbains. Elle pose donc question par rapport aux enjeux de mobilité puisque les localisations privilégiées sont généralement peu accessibles par les alternatives à la voiture. En outre, elle néglige les nouveaux lieux d'accueil des activités économiques et elle ne favorise pas l'attractivité urbaine alors que, comme nous l'avons constaté dans notre premier chapitre, cette ressource territoriale est de plus en plus considérée comme un facteur de compétitivité.

93 À l'image de la bonne santé financière d'une PME, il s'agit de termes définis par la loi du 15 mai 2014 portant exécution du pacte de compétitivité, d'emploi et de relance.

94 D'après l'arrêté royal du 22 octobre 2017 portant exécution, en ce qui concerne la Région wallonne, de l'article 16 de la loi du 15 mai 2014 portant exécution du pacte de compétitivité, d'emploi et de relance (Moniteur belge, 31 octobre 2017). 


\section{LA WALLONIE DANS LE CONTEXTE DE L'EUROPE DU NORD-OUEST}

Les géographes sont très créatifs en métaphores pour rendre compte des «lignes de force de l'espace " ${ }^{95}$, en particulier celles qui structurent l'économie du continent européen. $\mathrm{La}$ «banane bleue " ${ }^{96}$, la « grappe de raisins $" ~{ }^{97}$, le « ring ${ }^{98}$ sont ici autant de notions qui font référence à la mégalopole européenne, aussi appelée la dorsale européenne. Du Lancashire à la Toscane, cette dorsale concentre les plus fortes densités de grandes villes et les plus fortes productions de valeurs ajoutées au kilomètre carré. Historiquement, cette structuration de l'espace européen trouve son origine dans les courants commerciaux entre le pôle de l'Europe du Nord-Ouest et le pôle de la Vénétie-Lombardie-Toscane. Géographiquement, la dorsale est loin d'être homogène. Elle contient de nombreuses ruptures, principalement naturelles, parmi lesquelles les Alpes, mais également l'Ardenne.

Bien que la Wallonie soit positionnée au cour de la dorsale européenne, force est de constater que cette localisation ne s'accompagne pas mécaniquement d'une forte attractivité et d'un haut niveau de développement. Face à ce constat, il convient de s'interroger sur la manière dont cette région peut trouver sa place vis-à-vis des grandes aires métropolitaines qui l'entourent avec, d'une part, Bruxelles, Luxembourg et Lille à ses frontières et, d'autre part, Paris, Londres, la Randstad Holland et le bassin Rhin-Ruhr à proximité immédiate. La réponse n'est pas évidente car, pour fixer des générateurs de revenus, la Wallonie est en concurrence avec des régions voisines qui, elles aussi, bénéficient d'une localisation au cour de la dorsale. Les questions de l'attractivité et de la compétitivité restent donc posées dans un contexte où, rappelons-le, aucune ville wallonne ne représente une métropole complète de dimension européenne.

L'objectif de ce troisième chapitre est de situer le niveau de développement économique de la Wallonie et de ses provinces vis-à-vis de son environnement européen. Pour ce faire, nous comparerons cette région aux régions voisines de l'Europe du Nord-Ouest. Comparer la Wallonie aux régions qui l'entourent se justifie dans le contexte de continentalisation et de globalisation de l'économie productive. En effet, comme nous venons de le rappeler, la Wallonie est en concurrence avec les régions voisines pour capter des générateurs de revenus et, en particulier, les investissements directs étrangers (IDE) de la sphère productive.

Pour reprendre l'expression issue de R. BRUNET, «Lignes de force de l'espace européen », Mappemonde, volume 66, 2002, p. 14-19.

96 R. BRUNET (dir), Les villes européennes, op. cit.

97 K. Kunzmann, M. Wegener, «The pattern of urbanisation in Western Europe », Ekistics, volume 58, $\mathrm{n}^{\circ} 350-351,1991$, p. 282-291.

98 R. BRUNET, «Le Ring », L'Espace géographique, volume 27, n 4, 1998, p. 369. 
Pour les développements de ce chapitre, lorsque cela s'est avéré possible, nous avons privilégié l'échelle de traitement des régions NUTS 2 (à l'exception du premier traitement sur le temps de parcours pour atteindre $10 \%$ du PIB européen (cf. Carte 2), pour lequel nous avons privilégié l'échelle plus fine des régions NUTS 3). Pour rappel, l'échelle des régions NUTS 2 correspond notamment aux provinces belges et aux anciennes régions françaises ${ }^{99}$ (cf. supra, 2.1.2). L'avantage du traitement des données à ce niveau spatial est qu'il permet de positionner les grandes disparités socio-spatiales infra-wallonnes vis-à-vis de l'environnement européen.

Ce chapitre est structuré en deux sections. La première section décrit les environnements économiques de la Wallonie et de l'Europe du Nord-Ouest. La seconde section est consacrée à une analyse des disparités socio-spatiales.

\subsection{LES ENVIRONNEMENTS ÉCONOMIQUES DE LA WALLONIE ET DE L'EUROPE DU NORD-OUEST}

Afin de décrire les environnements économiques de la Wallonie et de l'Europe du NordOuest, nous allons maintenant nous intéresser à l'argument de la localisation « au cœur de l'Europe » ainsi qu'au sujet des niveaux de PIB et de PIB par habitant.

\subsubsection{L'argument de l'accessibilité privilégiée « au cœur de l'Europe»}

La notion d'accessibilité peut être définie comme la capacité d'un lieu à être atteint par d'autres lieux ou, inversement, par la capacité d'un lieu à donner accès à d'autres lieux. Argument de compétitivité régionale régulièrement mis en avant par les acteurs économiques wallons, la Wallonie bénéficierait d'une accessibilité privilégiée " au cœur de l'Europe ». Cela est censé représenter un avantage puisque l'accès aux marchés européens est un facteur de localisation souvent pris en compte par les firmes qui cherchent à sélectionner une implantation. À la réflexion, deux raisons majeures nous obligent à relativiser le poids de cet argument. Ces deux raisons correspondent au caractère relatif de la bonne accessibilité de la Wallonie ainsi qu'aux interrogations quant à l'impact effectif d'une localisation supposée centrale sur le développement économique.

Pour vérifier la qualité de l'accessibilité de la Wallonie, nous avons profité des travaux réalisés pour le programme de recherche ORATE ${ }^{100}$. Grâce aux données rassemblées pour ce programme, nous avons établi la carte 2 qui, à l'échelle des régions NUTS 3, rend compte du temps moyen nécessaire pour atteindre $10 \%$ du PIB de l'UE en utilisant soit la route, soit le chemin de fer, soit l'avion. Il ressort de l'analyse de cet indicateur d'accessibilité au marché européen que l'ensemble de la Wallonie n'est pas doté des mêmes

99 Avant l'entrée en vigueur du nouveau découpage régional au $1^{\mathrm{er}}$ janvier 2016.

100 L'Observatoire en réseau de l'aménagement du territoire européen (ORATE; en anglais, European Observation Network for Territorial Development and Cohesion - ESPON) est un programme de la politique régionale européenne. Il finance des analyses sur les dynamiques territoriales au niveau européen afin de fournir des recommandations aux décideurs politiques (cf. le site Internet www.espon.eu). 
niveaux d'accessibilité. Si le nord du sillon Sambre-et-Meuse est relativement proche de l'activité économique, cela est moins le cas au sud. Au nord du sillon, certains arrondissements affichent des valeurs élevées d'accessibilité qui permettent d'expliquer leur importante activité en logistique (notamment à Liège). Par contre, certains arrondissements ruraux affichent des valeurs comparables à d'autres territoires moins "au cœur de l'Europe », à l'image de la Normandie ou de la Bourgogne. La légende souligne ici des différences de temps pouvant atteindre $50 \%$, ce qui peut avoir un impact significatif sur les coûts de transport. De tels résultats poussent à s'interroger sur la possibilité de développer l'activité logistique dans toutes les sous-régions qui composent la Wallonie.

Il ressort également de la carte 2 que la Wallonie n'est pas la seule région européenne à pouvoir revendiquer une localisation « au cœur de l'Europe ». Des territoires situés en Flandre, aux Pays-Bas, dans l'ouest de l'Allemagne ou encore dans le nord de la France peuvent tout autant la revendiquer. La localisation centrale de la Wallonie ne représente donc pas un avantage différenciatif face à la concurrence des régions voisines.

Carte 2. Temps de parcours pour atteindre $10 \%$ du PIB européen en utilisant soit la route, soit le chemin de fer, soit l'avion (2011)

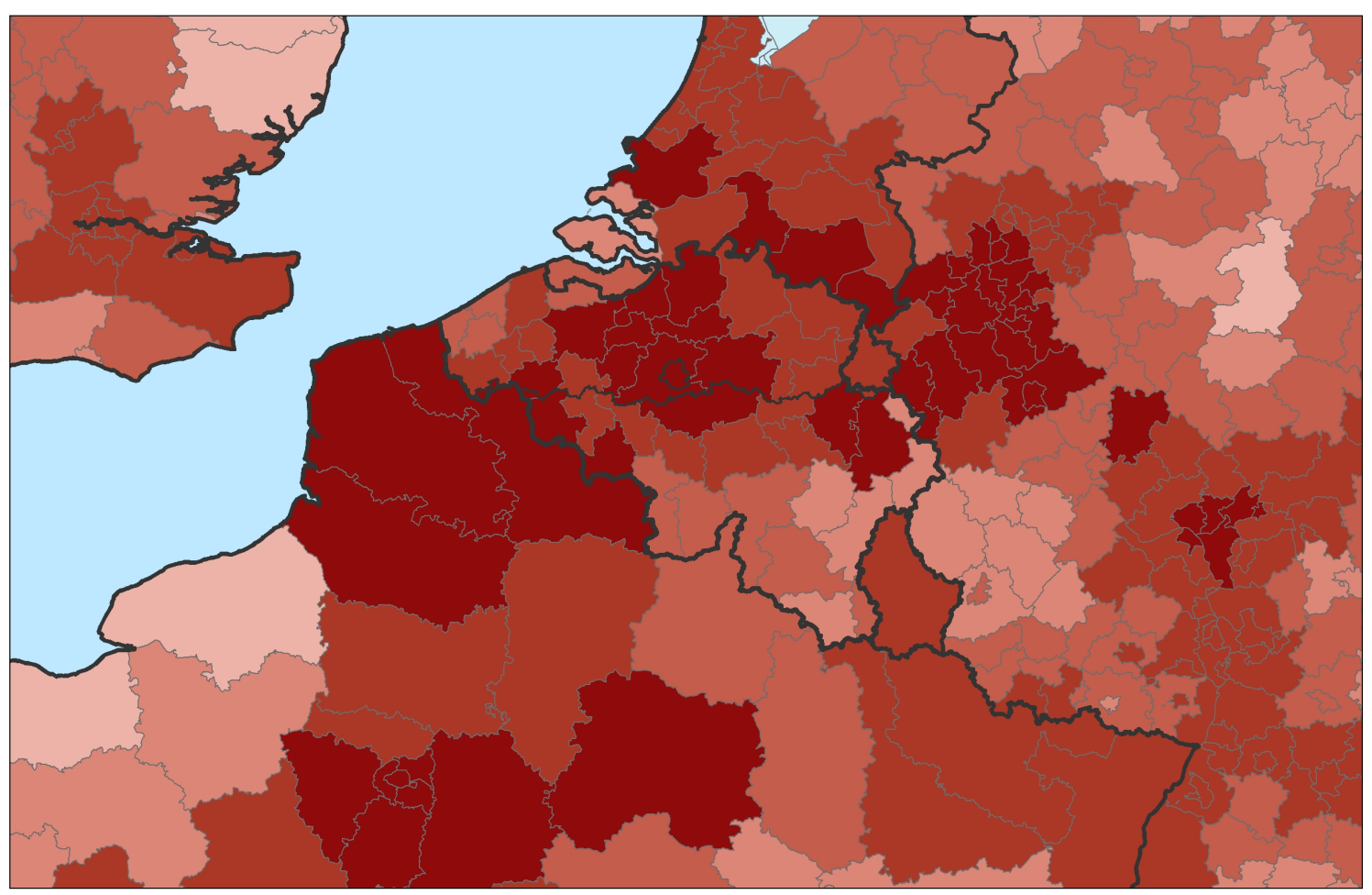

Moins de $2 \mathrm{~h} 20$

Entre $2 \mathrm{~h} 20$ et $2 \mathrm{~h} 40$

Entre $2 \mathrm{~h} 40$ et $3 \mathrm{~h} 00$

Entre $3 \mathrm{~h} 00$ et $3 \mathrm{~h} 20$

Entre $3 \mathrm{~h} 20$ et $3 \mathrm{~h} 40$

Plus de $3 \mathrm{~h} 40$

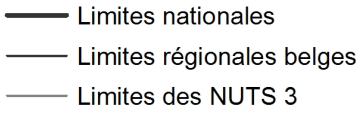

0

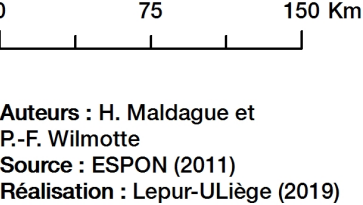


Une seconde raison qui justifie que l'argument de la position centrale doive être nuancé est que, comme nous l'avons discuté dans le premier chapitre, la centralité n'est pas forcément un gage de développement économique. En outre, dans un environnement économique où une concurrence est exercée par les territoires limitrophes, cette position peut même constituer une faiblesse. C'est potentiellement dans cette situation que se trouve la Wallonie, car elle est déforcée par d'autres facteurs que cette localisation « centrale » supposée favorable. Les territoires wallons voient ainsi passer des flux entre grands pôles économiques sans que les flux en question y induisent des retombées significatives. Face aux investissements et aux externalités auxquels il faudrait consentir pour y aboutir, la question reste d'ailleurs posée de savoir si la Wallonie a réellement intérêt à mobiliser des moyens importants afin de chercher à capter une partie de ces flux ${ }^{101}$.

$\mathrm{Si}$, comme nous venons de le voir, l'argument de la position centrale de la Wallonie en Europe se doit d'être relativisé, force est de constater qu'il n'est tout simplement pas valable s'il s'agit de desservir le marché belge. Lorsque l'on mesure l'accès au marché belge plutôt que l'accès au marché européen, Bruxelles et son hinterland flamand sont dotés des meilleures accessibilités. Or, en raison des besoins en conditionnements et étiquetages, les implantations des centres de distribution demeurent fortement déterminées par des logiques nationales. Il n'est donc pas étonnant que la région de Bruxelles-Capitale et la Flandre conservent l'essentiel des activités logistiques, comme le montrent les données sur les prises en occupation (take-up) du marché logistique belge ${ }^{102}$.

\subsubsection{Le PIB et le PIB par habitant}

Bien qu'ils ne permettent pas d'appréhender l'ensemble des dimensions du développement territorial ${ }^{103}$, nous ne pouvons pas nous permettre de négliger les indicateurs du PIB et du PIB/habitant. En effet, l'enjeu de la production de valeurs ajoutées demeure au cœur du paradigme du développement territorial. En outre, comme cela a été souligné dans l'introduction générale de ce Courrier hebdomadaire, la plupart des décideurs wallons continuent de baser leurs politiques sur l'idée selon laquelle la croissance du PIB est un préalable pour rencontrer les défis environnementaux et sociaux auxquels la Wallonie est confrontée.

La carte 3 porte sur la distribution du PIB et du PIB/habitant en Europe du Nord-Ouest. Pour le PIB/habitant, cette carte met en exergue les disparités entre États, notamment entre la France et l'Allemagne. Elle met également en exergue les niveaux de production élevés des grandes aires métropolitaines, en particulier l'Île-de-France. D'autres profils de régions se distinguent par des niveaux élevés de PIB/habitant, en particulier certaines régions industrielles reconnues pour leur potentiel d'innovation, à l'image du Bade-Wurtemberg en Allemagne ou du Brabant septentrional aux Pays-Bas. En bordure de la Wallonie,

101 Les débats autour de l'arrivée de la société chinoise Alibaba Groupe à Bierset illustrent ce questionnement (cf. par exemple la carte blanche collective "Alibaba, un projet d'avenir pour la Wallonie ? », Le Soir (en ligne), 30 avril 2019, https://plus.lesoir.be).

102 P.-P. VerELST, Logistics property in Belgium. Solid take-up volume in Q1 2016, Bruxelles, John Lang Lasalle, 2016.

103 C. RUYTERS et al., Indicateurs complémentaires au PIB : l'indice des conditions de bien-être en Wallonie ( $2^{e}$ exercice), op. cit. 
le Grand-Duché de Luxembourg et la Rhénanie, en l'occurrence les districts de Cologne et de Düsseldorf, affichent également de hauts niveaux de valeurs ajoutées par habitant.

En termes de PIB/habitant, le Brabant wallon se positionne très bien, à l'image de la région de Bruxelles-Capitale, du Brabant flamand et de la province d'Anvers, c'est-àdire les autres entités du cœur métropolitain de l'espace belge. À l'exception du Brabant wallon, le profil des provinces wallonnes est proche du profil des régions françaises voisines. Dans ces quatre provinces, la province de Liège se démarque par un PIB/habitant légèrement plus élevé.

Carte 3. PIB et PIB par habitant en standard de pouvoir d'achat (2014)

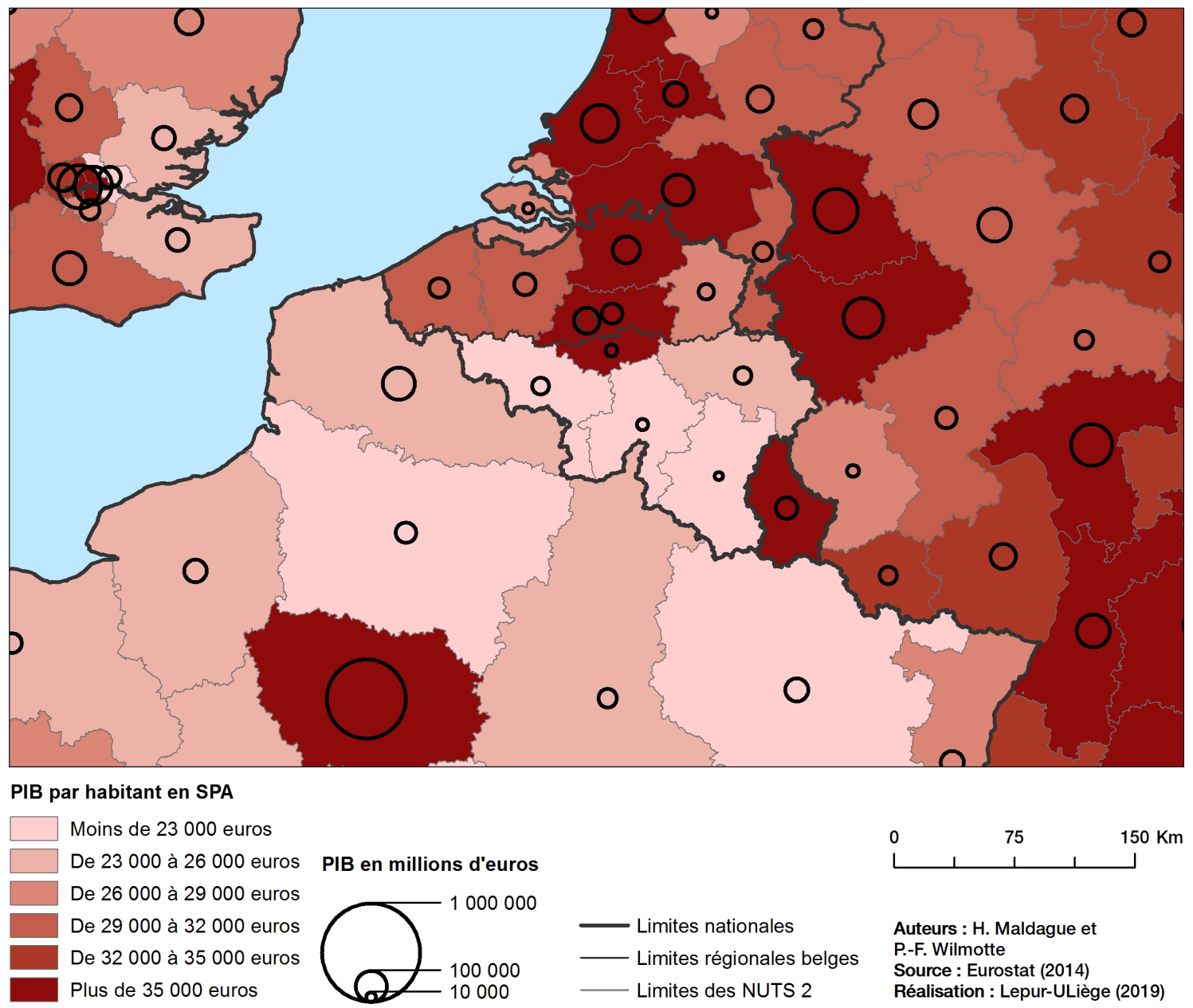

Quant à l'évolution sur les dix dernières années (cf. Carte 4), les impacts différenciés de la crise économique et financière déclenchée en 2008 semblent fortement influencer les trajectoires et la Belgique s'y caractérise par une situation intermédiaire entre l'Allemagne et la France. Cela étant, des disparités sensibles existent au niveau belge. Tout d'abord, si Bruxelles reste un moteur économique, la croissance récente bénéficie plus fortement aux provinces de Brabant wallon et de Brabant flamand qu'à la région de Bruxelles-Capitale. En dehors du Brabant wallon, le rythme de croissance des provinces wallonnes reste plus faible que celui des provinces flamandes, à l'exception notable de 
la province d'Anvers. En comparaison des régions frontalières, la carte 4 conduit toutefois à nuancer ce constat négatif, en situant le rythme moyen de croissance des provinces de Liège, de Namur et de Hainaut dans la moyenne de la zone d'étude. La situation de la province de Luxembourg est par contre moins favorable, ce qui est vraisemblablement lié à la concurrence exercée par le Grand-Duché de Luxembourg sur les investissements productifs (cf. notamment infra, 6.2).

Carte 4. Évolution du PIB en standard de pouvoir d'achat entre 2005 et 2014

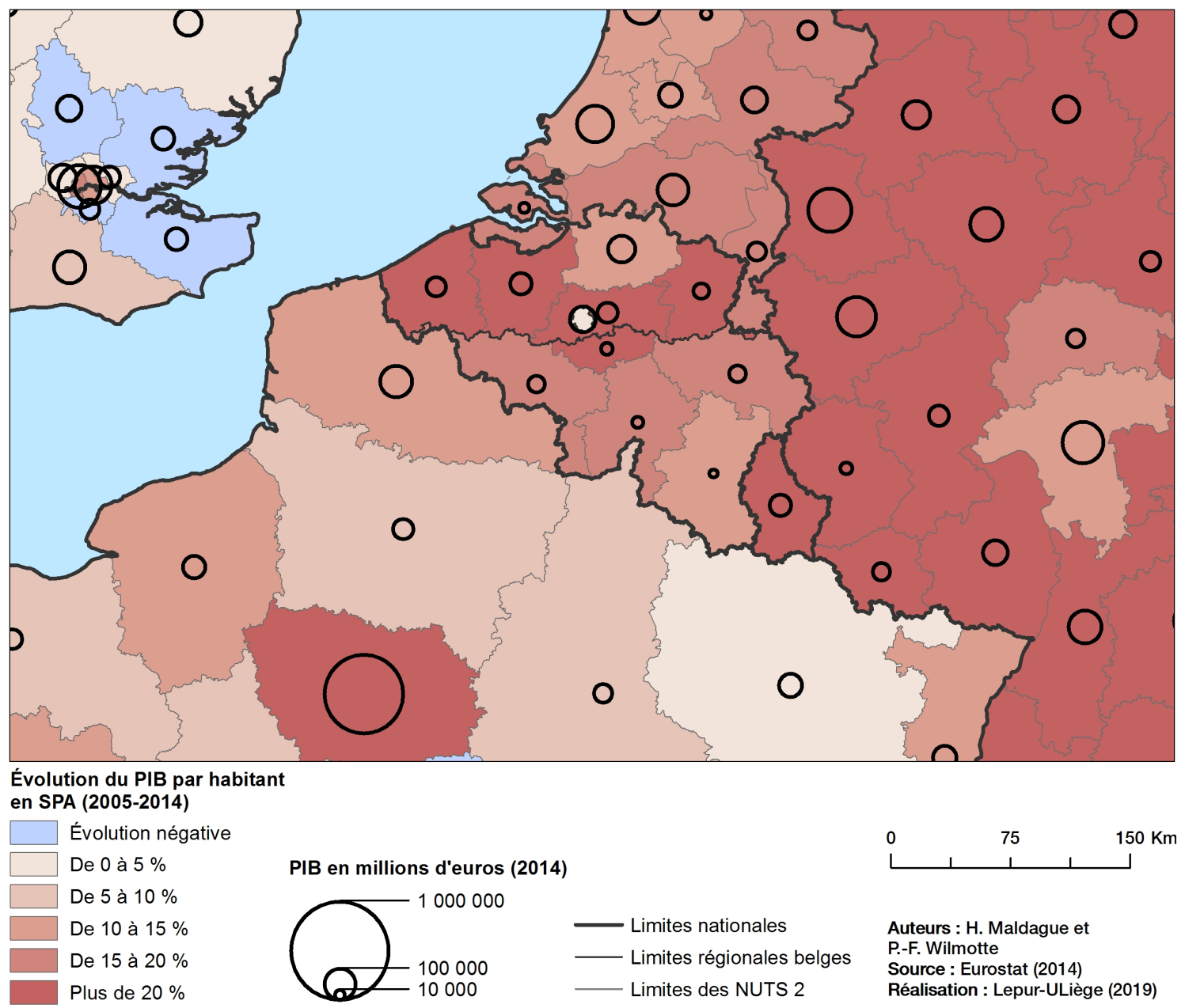

\subsection{LES DISPARITÉS SOCIO-SPATIALES EN EUROPE DU NORD-OUEST À LA LUMIÈRE DES OBJECTIFS DE LA STRATÉGIE EUROPE 2020}

Les éléments développés dans la section précédente ont permis de décrire les principales caractéristiques des environnements économiques en Wallonie et en Europe du NordOuest. Nous allons maintenant nous appuyer sur ce panorama pour une analyse basée sur les objectifs de la stratégie Europe 2020. Cette stratégie se base sur huit indicateurs de référence (cf. supra, 2.1.1). Parmi ces huit indicateurs, quatre concernent directement 
la question des disparités socio-spatiales en termes de développement territorial. Il s'agit du taux d'emploi, de la part du PIB investie en R\&D, de la part des diplômés de l'enseignement supérieur chez les 30-35 ans et du taux de sortie précoce du système scolaire.

Pour chacun des indicateurs, des objectifs ont été fixés en 2010 pour l'horizon 2020. Compte tenu des disparités socio-spatiales au sein de l'UE, ces objectifs sont déclinés en fonction des réalités et des stratégies nationales. Pour la Belgique, les objectifs fixés par la Commission européenne et les autorités du pays correspondent à 73,2 \% pour le taux d'emploi, à 3,0 \% pour la part du PIB investie en $\mathrm{R} \& \mathrm{D}$, à $47,0 \%$ pour la part de diplômés de l'enseignement supérieur chez les 30-35 ans et à 9,5\% pour le taux de sortie précoce du système scolaire (cf. Tableau 2).

La suite de cette section est structurée en trois sous-sections. Au point 3.2.1, nous décrivons les faits saillants d'une approche basée sur un indice composite établi à partir des indicateurs que nous venons de rappeler. Au point 3.2.2, nous approfondissons l'analyse par une réflexion spécifique sur chacun des quatre indicateurs. Au point 3.2.3, nous synthétisons les principales caractéristiques des cinq provinces wallonnes.

\subsubsection{L'indice composite de suivi des objectifs du programme Europe 2020}

Nous venons de rappeler que la stratégie Europe 2020 se base sur une série d'indicateurs. Parmi ces indicateurs, quatre se rapportent directement à la problématique du développement territorial. Afin d'étudier le développement de la Wallonie à la lumière des objectifs de la stratégie Europe 2020, nous avons choisi de considérer ces quatre indicateurs en les intégrant dans un indice composite. Pour calculer cet indice composite, nous nous sommes inspirés de la méthode appliquée en 2010 par la Commission européenne dans l'évaluation de la position des régions européennes vis-à-vis des principaux objectifs de la stratégie de Lisbonne ${ }^{104}$.

\subsubsection{1. Élaboration de l'indice composite}

Sur le plan méthodologique, l'indice composite repose sur quatre indices spécifiques calculés pour l'ensemble des régions NUTS 2 des États membres de l'UE. Les indices spécifiques sont établis à partir des indicateurs par une règle de trois où, pour chaque indicateur, les régions NUTS 2 obtiennent un score compris entre 0 point et 100 points. Le score de 0 point est attribué à la région européenne avec le plus faible résultat. À l'autre bout du spectre, le score de 100 points est attribué aux régions ayant atteint leur objectif fixé pour le niveau européen. Ensuite, pour établir l'indice composite, une moyenne est calculée à partir des quatre indices spécifiques.

104 Commission Européenne, «Investir dans l'avenir de l'Europe. Cinquième rapport sur la cohésion économique, sociale et territoriale », Luxembourg, Office des publications de l'Union européenne, 2010, p. 195-196. 
Le résultat de cette moyenne peut être appréhendé comme un score global évaluant le suivi des objectifs assignés. Un score de 0 point signifierait que la région concernée est la dernière entité de l'UE pour les quatre indicateurs. A contrario, lorsque le score de 100 points est atteint, cela signifie que les quatre objectifs assignés ont été rencontrés. Bien sûr, à l'image de toute construction statistique, la méthodologie appliquée n'est pas exempte de limites. Elle présente toutefois le double avantage d'obtenir un indice qui, d'une part, tienne compte de différentes facettes du développement territorial et qui, d'autre part, repose sur une légitimité politique puisque les calculs se basent sur les objectifs fixés par les autorités européennes.

La construction des indices a été élaborée à partir des données les plus récentes disponibles lors des traitements, à savoir 2015. L'indice composite peut être utilisé pour évaluer une situation à un instant donné, mais également pour suivre une évolution. Nous présentons ci-dessous une évolution pour la période $2010-2015^{105}$. Pour l'évolution, les résultats mitigés auxquels nous aboutissons pour les provinces wallonnes sont sans doute partiellement liés à des effets de conjoncture. En effet, si l'économie wallonne a globalement bien résisté à la crise économique et financière déclenchée en 2008, le redémarrage de la première moitié des années 2010 y a été plus lent qu'ailleurs.

\subsubsection{La Wallonie vis-à-vis des autres régions de l'Europe du Nord-Ouest}

La carte 5 représente l'indice composite tel que calculé sur la base de la situation de 2015 . En complément, la carte présente également l'évolution de l'indice sur la période 2010-2015.

Au sein de la zone représentée et pour l'année 2015, seule la région anglaise du Bedfordshire and Hertfordshire atteint l'ensemble des objectifs assignés. Bon nombre d'autres régions NUTS 2 tendent toutefois vers le score de 100 points (cf. la catégorie comprise entre 94 points et 99,9 points). Ces régions NUTS 2 pouvant être considérées comme des régions compétitives appartiennent à deux groupes.

Il s'agit, premièrement, de régions qui bénéficient des retombées de capitales nationales ou de métropoles de niveau international, cela via le développement de l'économie productive mais aussi via l'accueil de travailleurs qualifiés. L'Île-de-France, le Brabant wallon et les provinces néerlandaises qui constituent la Randstad s'inscrivent dans ce modèle. La région de Bruxelles-Capitale, par contre, correspond à un indice composite peu enviable de 71,8 points. Méthodologiquement, cela tient à sa superficie très limitée en comparaison des autres régions NUTS 2 . Il résulte de cette superficie très limitée que, statistiquement parlant, la paupérisation des milieux urbains n'y est pas diluée par le caractère généralement plus favorisé des zones périurbaines.

Parmi les régions bien classées, on trouve aussi des régions ayant développé des filières économiques compétitives, à l'image des régions précédemment citées du BadeWurtemberg allemand ou du Brabant septentrional néerlandais. Les dynamiques de ce second groupe de régions s'inscrivent dans le modèle des économies de localisation (ou de juxtaposition), alors que le succès des territoires irrigués par les dynamiques des capitales s'inscrit davantage dans le modèle des économies d'urbanisation (cf. supra, 1.1.1).

105 À l'exception toutefois des données françaises relatives à la part du PIB investie en R\&D, qui datent pour leur part de 2013. 
Carte 5. Régions européennes NUTS 2 par rapport à l'indice composite du suivi des objectifs du programme Europe 2020 (2015)

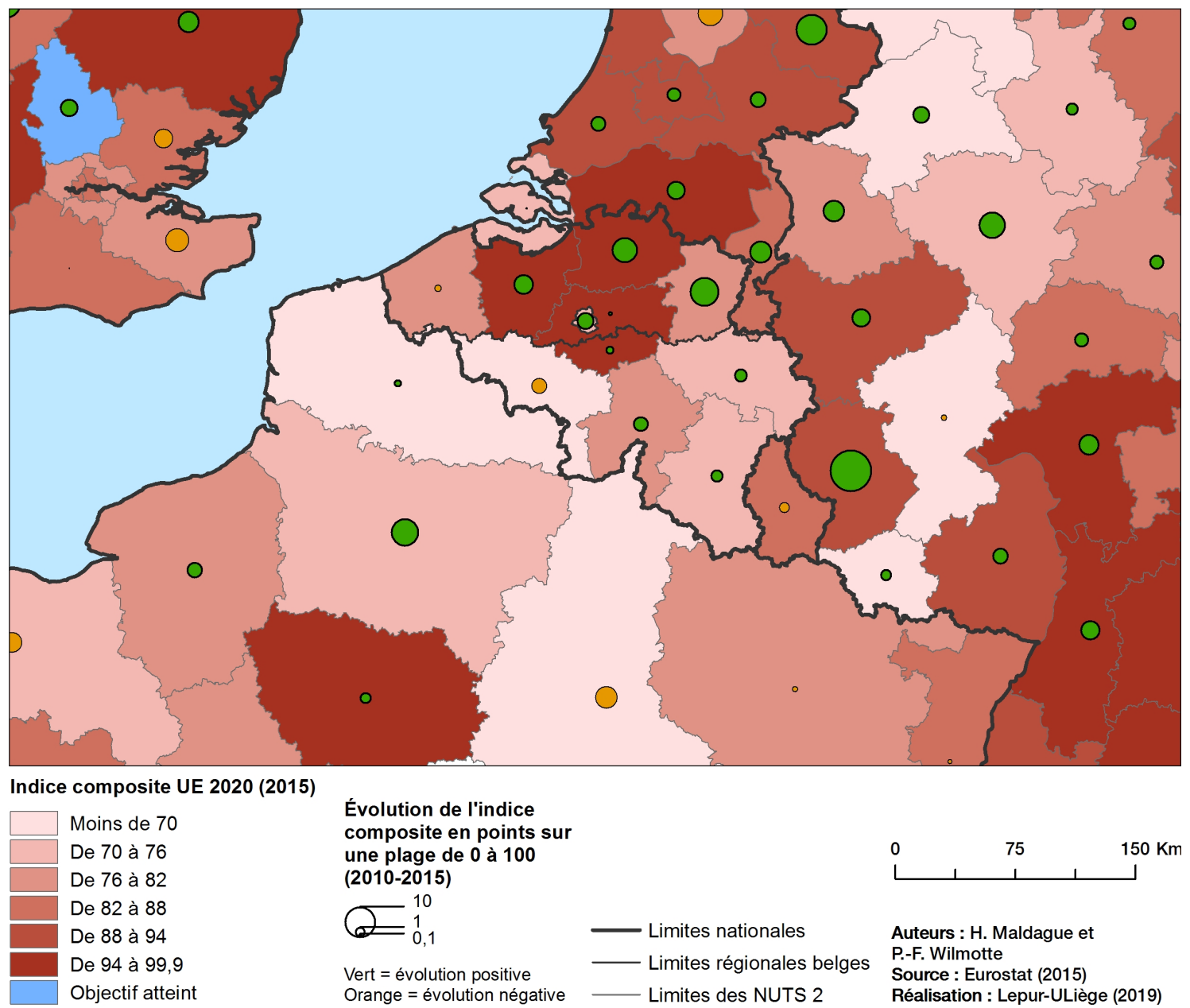

À l'exception du Brabant wallon (95,4 points), les provinces wallonnes n'appartiennent pas aux catégories des régions européennes compétitives, avec des indices qui se situent tous sous la barre des 80 points : 63,5 pour le Hainaut, 70,4 pour le Luxembourg, 74,6 pour la province de Liège et 78,3 pour la province de Namur. Vis-à-vis des provinces flamandes, on trouve en Wallonie des indices plus faibles. En outre, à l'exception de la Flandre occidentale, l'évolution sur la période 2010-2015 a été est moins favorable en Wallonie qu'en Flandre. Pour le Hainaut, nous trouvons même une évolution négative. Mathématiquement, le caractère négatif ne signifie pas nécessairement que les indicateurs absolus soient en décroissance. Par contre, une évolution négative implique que la progression vers les objectifs affichés est moins rapide qu'au sein des régions européennes les moins bien classées.

Dans la zone étudiée, les évolutions négatives les plus fortes sont observées au sein du Kent anglais, de la province néerlandaise de Flevoland et de la Champagne-Ardenne française. Une plausible explication à ce constat peut résider dans le fait que ces régions soient pénalisées par la conjonction d'une armature urbaine faible et de la proximité 
à une métropole majeure - respectivement Londres, Amsterdam et Paris - qui les concurrence et dont elles ne parviennent pas à tirer profit.

\subsubsection{La Wallonie vis-à-vis des autres régions de tradition industrielle}

Afin de compléter l'analyse de l'indice composite, sortons du cadre de l'Europe du Nord-Ouest pour comparer les provinces wallonnes à d'autres régions européennes de tradition industrielle (RETI). Rappelons que les RETI sont des territoires qui se sont industrialisés durant la première ou durant la deuxième révolution industrielle, à partir principalement d'activités minières, textiles ou sidérurgiques. Comparer les provinces wallonnes à ce groupe de régions permet de vérifier dans quelle mesure leurs trajectoires de développement convergent ou s'écartent des trajectoires de développement des autres territoires européens ayant connu une industrialisation précoce et une histoire économique comparable. Â ce titre, les comparaisons sont particulièrement pertinentes pour les provinces de Liège et de Hainaut, qui sont les deux provinces les plus marquées par les industries traditionnelles et qui, en même temps, continuent de représenter un poids considérable dans l'économie wallonne.

Pour la sélection des RETI, nous nous sommes basés sur la liste établie par Henri Capron en $2009^{106}$, liste que nous avons ajustée en fonction du découpage NUTS 2. Nous avons aussi choisi de sélectionner le Limbourg néerlandais pour son passé industriel dans la Parkstad Limburg, ainsi que le Pays basque espagnol, dont le modèle de redéploiement économique fait référence ${ }^{107}$.

Le graphique 1 porte sur l'indice composite des provinces wallonnes et des RETI étrangères. Il permet de voir à quel point l'indice pour le Brabant wallon s'écarte des indices des autres provinces wallonnes, mais aussi de ceux des autres régions prises en compte. Quant aux résultats des quatre autres provinces wallonnes, ils se situent globalement dans la moyenne des autres régions, à l'exception toutefois du Hainaut, qui représente le deuxième indice le plus faible après la région espagnole des Asturies.

Le graphique 1 témoigne des grandes disparités socio-spatiales entre les provinces wallonnes mais aussi, à l'exception du Royaume-Uni, des grandes disparités entre RETI appartenant à un même État. Ces disparités témoignent qu'il n'y a pas de solution miracle pour redéployer l'économie d'une région de tradition industrielle.

Nous reviendrons en détail sur les disparités entre les provinces wallonnes dans la section suivante, consacrée à l'analyse des quatre indicateurs de base. Toutefois, précisons dès à présent que le meilleur positionnement des provinces de Namur et de Liège repose sur l'influence de leurs chefs-lieux en tant que pôle administratif et universitaire. Cela a un impact en particulier sur la variable de la part des personnes de 30-35 ans qui disposent d'un diplôme de l'enseignement supérieur.

106 H. CAPRON, "La compétitivité des régions », Reflets et perspectives de la vie économique, volume 48, $\mathrm{n}^{\circ} 1-2,2009$, p. 115-136.

K. MORGAN, "Collective entrepreneurship: the Basque model of innovation », European Planning Studies, volume $24, n^{\circ} 8$, p. 1544-1560. 
Graphique 1. Classement des RETI selon l'indice composite de suivi des objectifs Europe 2020

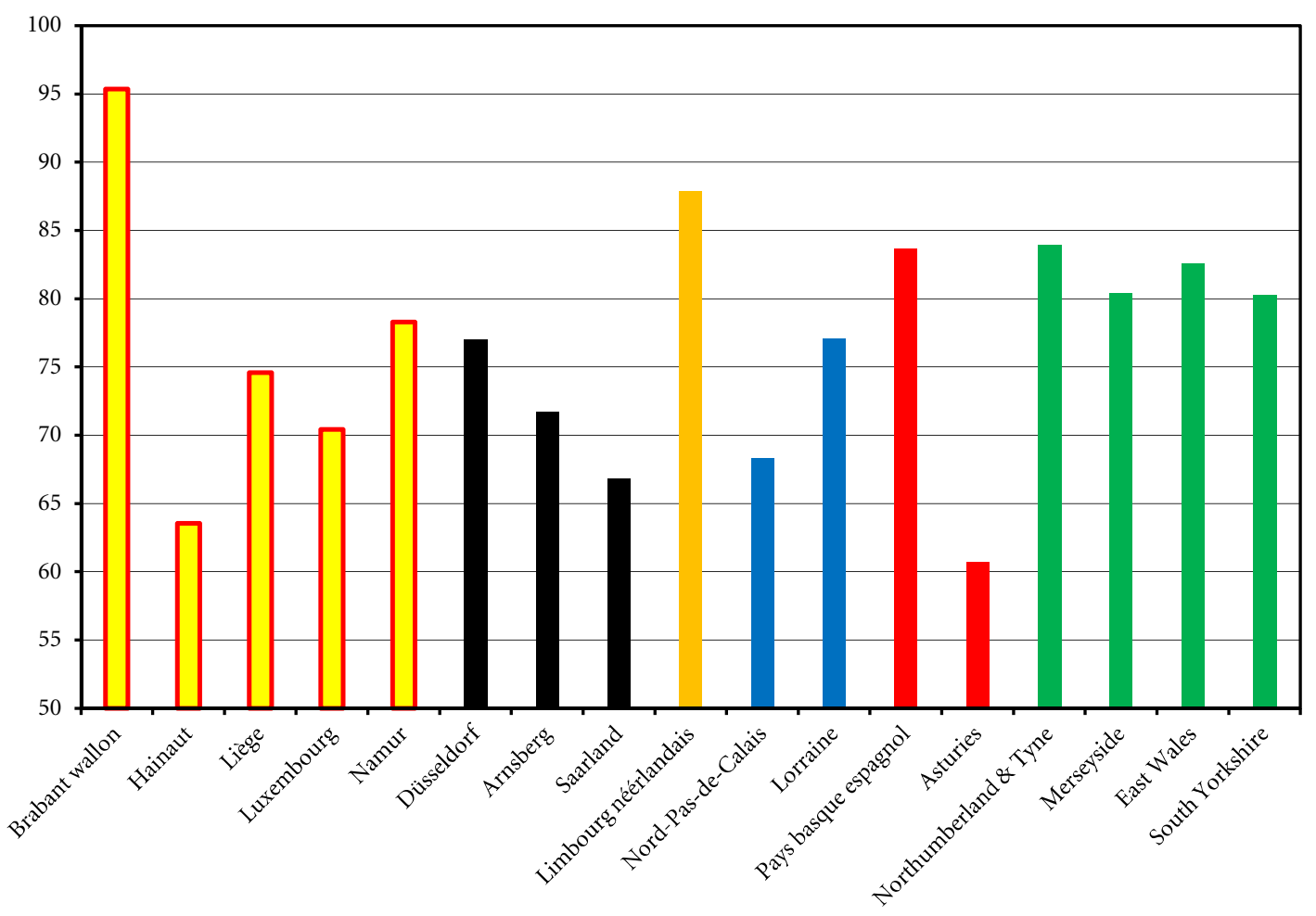

Sources : Commission européenne, 2010 ; ESPON, 2013 ; Eurostat, 2016.

Le graphique 2 porte sur l'évolution de l'indice composite entre 2010 et 2015. La situation de la province de Hainaut y apparaît très problématique. En effet, elle connait l'évolution la plus défavorable parmi les RETI considérées.

En comparaison du graphique 1, l'effet lié aux appartenances nationales se marque de manière plus structurelle sur le graphique 2 . Les évolutions les plus positives y sont observées au Royaume-Uni (à l'exception de l'entité East Wales), en Allemagne et aux Pays-Bas. Pour le Royaume-Uni et les Pays-Bas, il est vraisemblable que cela résulte d'un effet rebond suite à l'impact de la crise économique et financière déclenchée en 2008. L'évolution est moins favorable en France et surtout en Espagne, qui a vécu des moments difficiles avec la crise de la dette. Pour les deux régions espagnoles considérées, cela s'est traduit par une réduction des capacités d'investissement en R\&D et par une hausse du chômage (et donc une baisse du taux d'emploi). 
Graphique 2. Évolution de l'indice composite de suivi des objectifs Europe 2020 entre 2010 et 2015

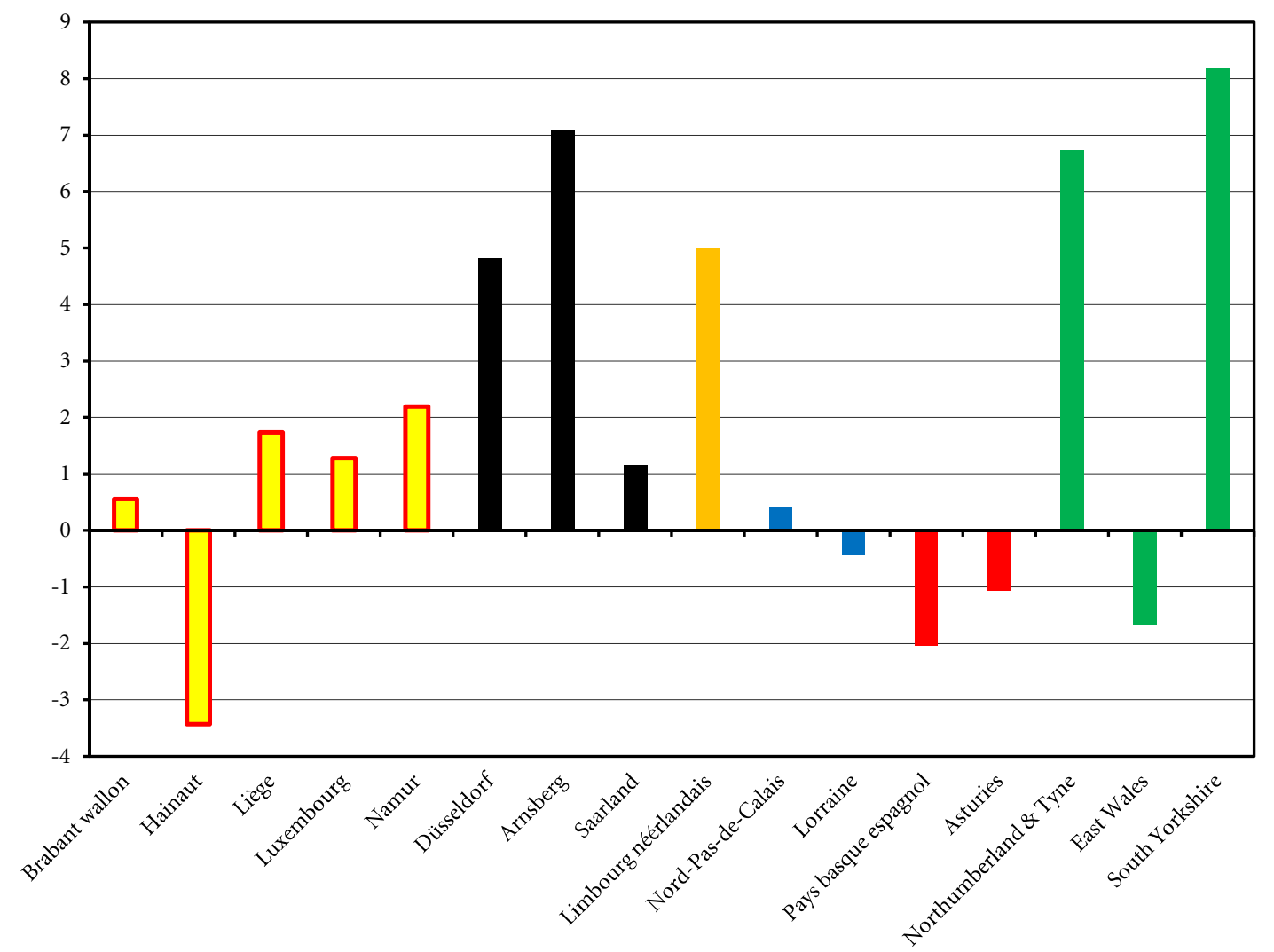

Sources : Commission européenne, 2010 ; ESPON, 2013 ; Eurostat, 2016.

\subsubsection{Analyse des composantes de l'indice composite}

À la suite de l'approche basée sur l'indice composite, nous pouvons maintenant approfondir l'analyse par une réflexion sur chacun des quatre indicateurs pris en compte dans sa construction.

\subsubsection{Le taux d'emploi}

Débutons la description des composantes de l'indice composite par le taux d'emploi. Les objectifs nationaux fixés par le programme Europe 2020 pour le taux d'emploi varient sensiblement (cf. Tableau 2). Pour rappel, l'objectif a été fixé à $75 \%$ pour l'échelle européenne, mais à $80 \%$ pour les Pays-Bas et à $73 \%$ pour le Grand-Duché de Luxembourg. Entre ces deux extrêmes, les objectifs assignés sont de 73,2\% pour la Belgique, de $75 \%$ pour la France et de $77 \%$ pour l'Allemagne.

Trois provinces flamandes parviennent à atteindre l'objectif des 73,2 \% (cf. Carte 6). La situation est plus critique en Wallonie, en particulier dans les provinces de Hainaut (57,6\%) et de Liège $(60,7 \%)$, dont on sait combien elles ont été affectées par le déclin industriel. 
Carte 6. Taux d'emploi : écart aux objectifs nationaux du programme Europe 2020 (2015) et évolution entre 2005 et 2015

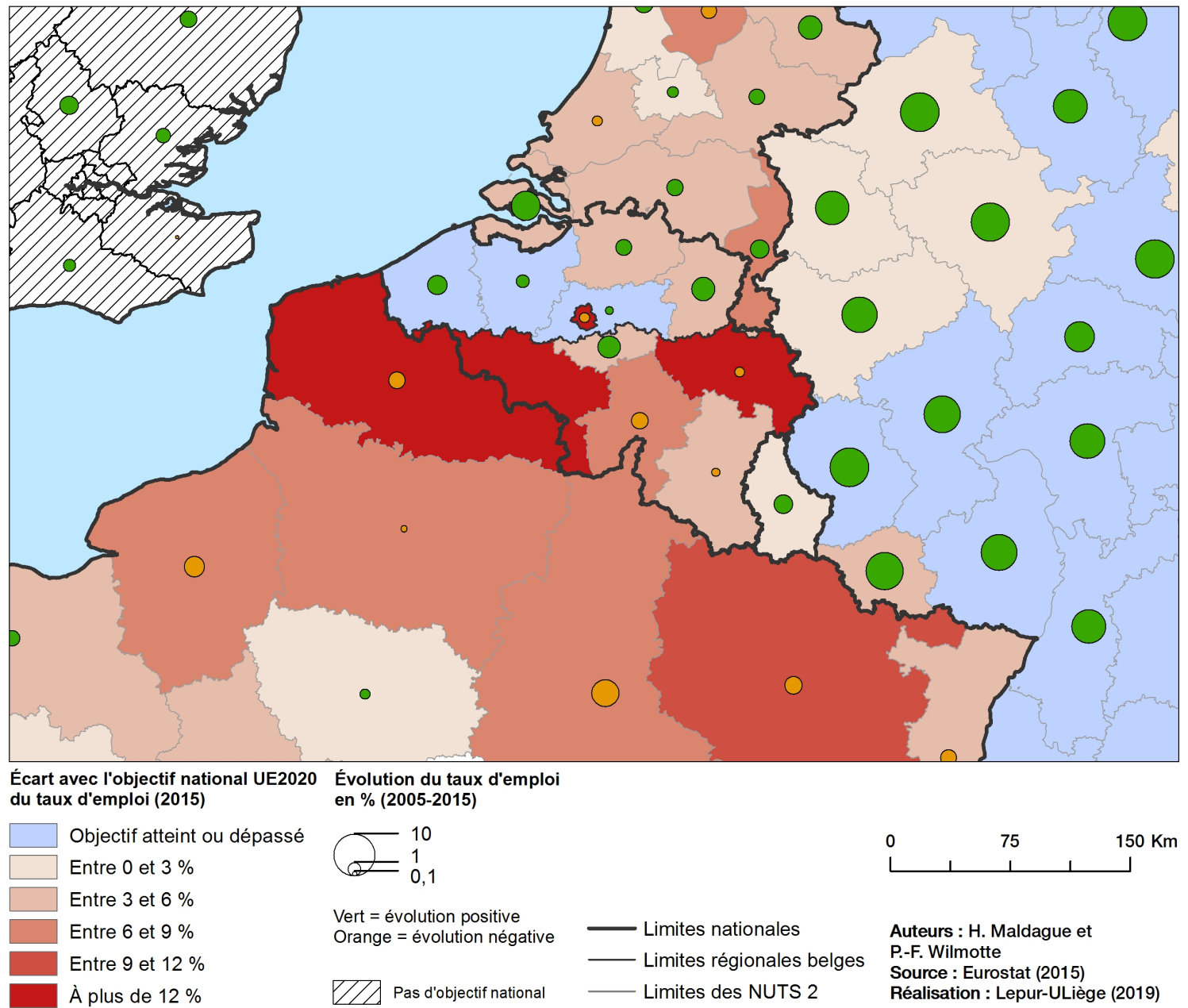

Au sein de l'espace pris en compte, on trouve deux autres entités qui, à l'instar des provinces de Hainaut et de Liège, accusent un différentiel de plus de $12 \%$ entre le taux d'emploi effectif et le taux d'emploi souhaité : la région de Bruxelles-Capitale et le Nord-Pas-de-Calais. Bruxelles est confrontée à un problème structurel bien connu d'inadéquation entre les emplois offerts sur le territoire et les qualifications des populations résidentes. Pour le Nord-Pas-de-Calais, qui appartient aussi à la catégorie des RETI, l'important écart trouve son origine dans le déclin industriel.

L'évolution du taux d'emploi entre 2005 et 2015 est fortement marquée par les appartenances nationales. Les contrastes sont très frappants entre les régions françaises et les régions allemandes. Quoique moins nettement que les régions allemandes, les provinces flamandes, ainsi que la plupart des régions britanniques et néerlandaises, présentent aussi des évolutions positives. En Wallonie, seule la province de Brabant wallon bénéficie d'une évolution positive du taux d'emploi. Le taux d'emploi a par contre stagné dans le Hainaut (ce qui explique que le cercle proportionnel ne soit pas visible sur la carte 6) et diminué dans les trois autres provinces wallonnes. La similitude 
est ici frappante entre la situation wallonne et la situation de la plupart des régions françaises considérées (à l'exception de l'Île-de-France).

Au final, le taux d'emploi ressort clairement comme le talon d'Achille de la Wallonie : d'une part, les taux atteints en 2015 sont bien en dessous des objectifs assignés et, d'autre part, l'évolution entre 2010 et 2015 est globalement défavorable. En conséquence, l'évolution du taux d'emploi pénalise fortement l'évolution globale de l'indice composite.

\subsubsection{La part du PIB consacrée à la recherche et développement}

La carte 7 porte sur la part du PIB investie dans le secteur de la R\&D. Parmi les voisins de la Belgique, peu de régions ont franchi les seuils mis en avant par la stratégie Europe 2020 (3 \% pour l'Union européenne, la Belgique, l'Allemagne et la France ; 2,5 \% pour le Grand-Duché de Luxembourg et les Pays-Bas). Il s'agit de régions au nord de Londres - où l'on trouve notamment les deux universités d'Oxford et de Cambridge - ainsi que de régions allemandes (principalement dans le Bade-Wurtemberg). En Belgique, la barre des $3 \%$ est dépassée dans trois provinces : Anvers, le Brabant flamand et le Brabant wallon.

Carte 7. Part du PIB consacrée à la R\&D (2015)

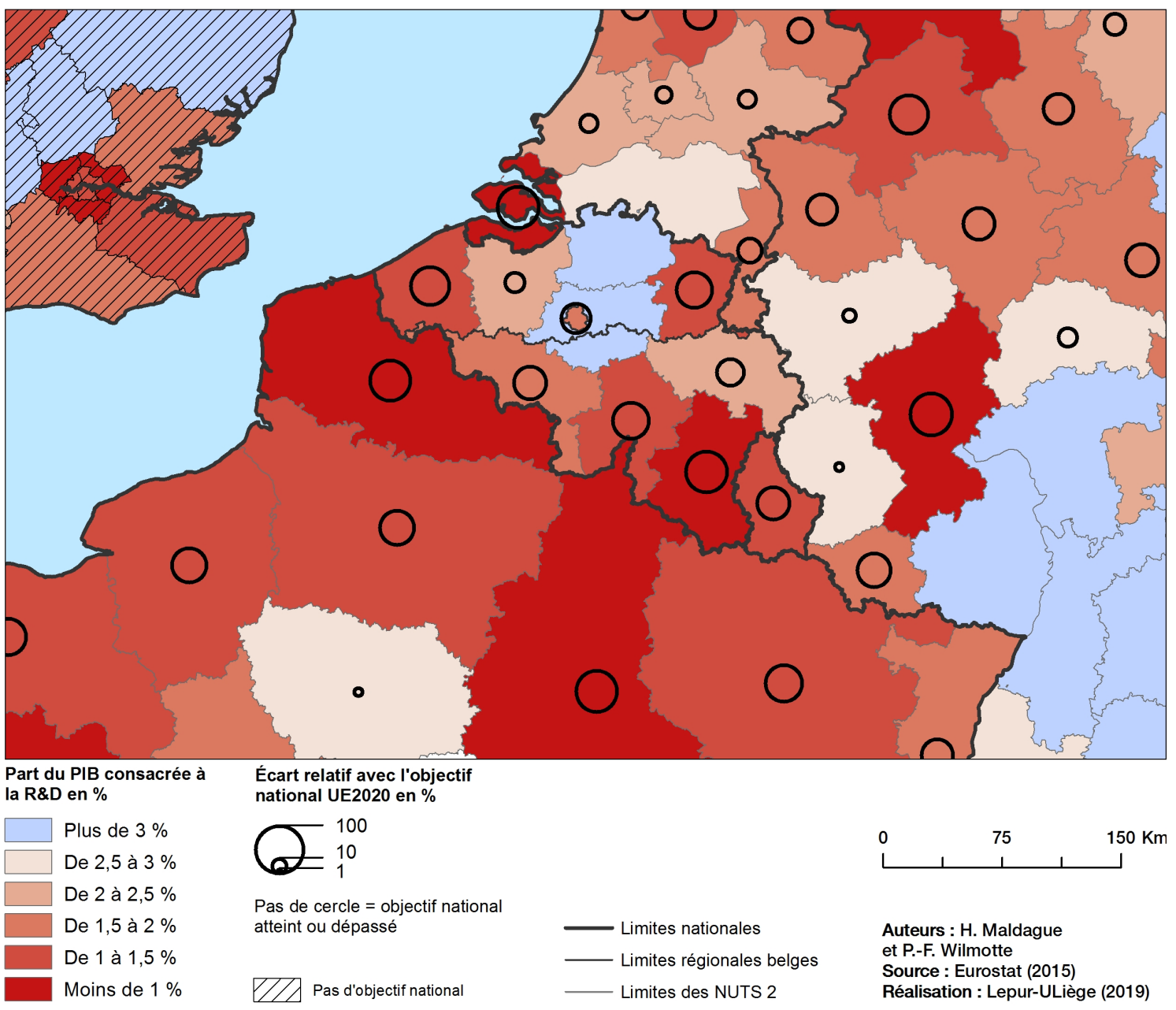


En Wallonie, les provinces de Namur et de Luxembourg affichent des taux inférieurs à la valeur de 1,5\%. Ces territoires, plutôt ruraux, disposent de peu de centres de recherche et de peu d'entreprises avec une forte intensité en R\&D. Pour les provinces de Liège et de Hainaut, la situation est intermédiaire entre les régions rurales à faible $R \& D$ (par exemple, la province néerlandaise de Zélande ou la Champagne-Ardenne française) et les régions où l'indicateur affiche des valeurs proches de l'objectif européen (comme la province néerlandaise de Brabant septentrional ou comme la Flandre orientale).

\subsubsection{La part de diplômés de l'enseignement supérieur chez les 30-35 ans}

À l'image de la composante du taux d'emploi, la composante de la part de la population diplômée de l'enseignement supérieur chez les 30-35 ans se caractérise par une forte variabilité des objectifs nationaux. À l'échelon européen, la barre a été fixée à $40 \%$, mais elle a été fixée plus haut au Grand-Duché de Luxembourg (66\%), en France (50\%), en Belgique (47\%) et en Allemagne (42\%). Par contre, le seuil des $40 \%$ s'applique également aux Pays-Bas.

L'indicateur repris sur la carte 8 trace de larges disparités aux échelles tant nationales que régionales. Certaines disparités entre pays sont induites par des différences entre systèmes éducatifs. Cela explique en particulier la faiblesse structurelle des régions allemandes, où la formation professionnelle ou continue est plus développée ${ }^{108}$. Malheureusement, ces différences ne manquent pas d'introduire des difficultés dans l'interprétation des données statistiques.

À l'échelon des pays, les régions des capitales et des grandes métropoles affichent généralement une part importante de diplômés de l'enseignement supérieur. Cela est manifeste au niveau de l'île-de-France, de la Randstad Holland et de Londres. En Belgique, cela se traduit par des parts de plus de $50 \%$ au sein des provinces de Brabant wallon et de Brabant flamand. Ces résultats peu surprenants corroborent le constat avancé dans l'état de l'art selon lequel les niveaux de qualification tendent à augmenter avec la taille des régions urbaines.

En Wallonie, c'est le Brabant wallon et la province de Namur qui bénéficient des parts de diplomation les plus importantes. Ces deux provinces cumulent les deux avantages de compter des villes universitaires et de représenter des zones résidentielles attractives pour les travailleurs qui occupent les emplois qualifiés du bassin économique de Bruxelles. Par contre, les parts les plus faibles sont observées au sein des provinces de Hainaut et de Luxembourg. Ces provinces sont de plus en plus attractives suite à la pression immobilière des métropoles proches, mais cela ne suffit pas pour contrebalancer une offre en enseignement supérieur relativement limitée ${ }^{109}$. Par rapport aux autres provinces wallonnes, la province de Liège occupe une position intermédiaire, avec un profil proche des régions du nord-est de la France. L'offre en enseignement supérieur y est importante, mais ce territoire demeure pénalisé par une attractivité urbaine faible à l'échelle européenne.

108 M. Duru-Bellat, A. KiefFer, I. Mearelli-Fournier, « Le diplôme, l'âge et le niveau : sens et usage dans les comparaisons de systèmes éducatifs ", Sociétés contemporaines, volume 26, n 2, 1997, p. 45-72.

109 Concernant la province de Luxembourg, il faut préciser que l'indicateur apparaît peu robuste au vu de la variabilité interannuelle (l'indicateur affiche par exemple 41,0 \% en 2016, contre 34,1\% en 2015). Cela s'explique parce que les résultats sont établis à partir d'enquêtes et que le faible volume de population de la province de Luxembourg s'accompagne d'une représentativité statistique limitée. 
Carte 8. Part des 30-35 ans avec un diplôme de l'enseignement supérieur (2015)

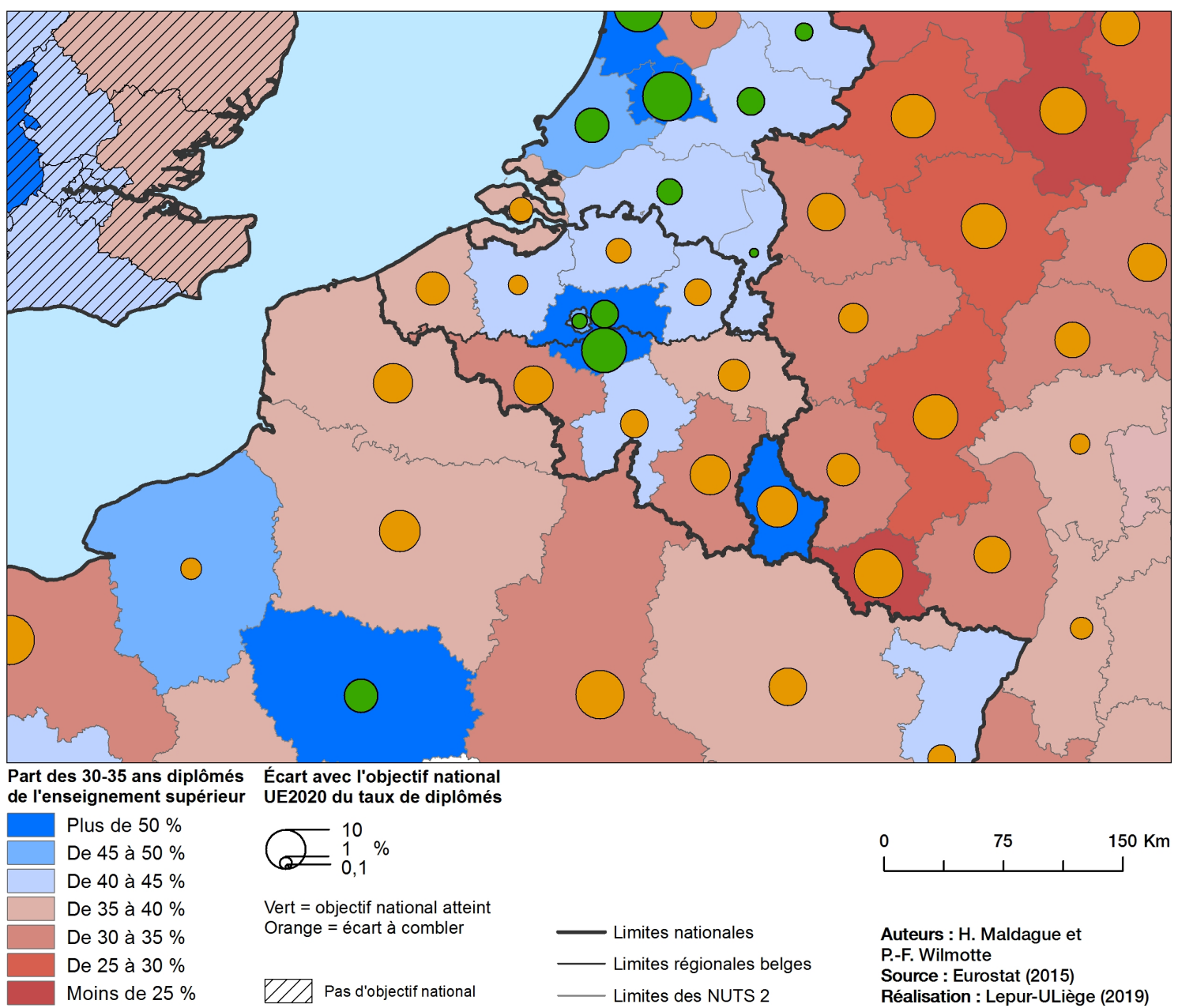

\subsubsection{Le taux d'abandon scolaire précoce}

La carte 9 représente le taux d'abandon scolaire précoce (ASP). Cet indicateur est mesuré par la proportion des jeunes entre 18 et 24 ans qui ont quitté l'école en n'ayant achevé que l'enseignement secondaire inférieur (ou moins) et qui ne poursuivent ni étude ni formation. Pour le niveau européen, l'objectif de la stratégie Europe 2020 est ici fixé à la barre des $10 \%$. Pour la Belgique, l'ambition est un peu plus élevée, avec un seuil fixé à $9,5 \%$.

La carte 9 met en lumière de fortes disparités au sein de la Wallonie. Les provinces de Brabant wallon et de Luxembourg ont atteint l'objectif européen, alors que les provinces de Liège et de Hainaut dépassent les 14 \% d'abandon scolaire précoce. Au sein de la zone d'étude, ce seuil n'est dépassé que par la région de Bruxelles-Capitale (non visible sur la carte) et par la province néerlandaise de Zélande. Pour sa part, la province de Namur correspond à une situation intermédiaire.

La question de l'ASP oblige à souligner la problématique du capital humain au sein des RETI, une problématique déjà discutée dans notre premier chapitre. Les données mobilisées indiquent que des difficultés particulièrement importantes caractérisent les 
systèmes éducatifs des bassins de tradition industrielle. Des difficultés importantes sont également rencontrées par la région de Bruxelles-Capitale, mais elles sont par contre moins marquées dans les territoires wallons aux caractéristiques plus rurales. Ces observations sur le problème de la relégation sont à mettre en lien avec le constat bien connu selon lequel, en raison notamment d'une culture persistante de l'élitisme, le système scolaire belge francophone est particulièrement inéquitable ${ }^{110}$.

Concernant l'ASP, il est utile de compléter l'analyse par un constat positif. En effet, cet indicateur est structurellement à la baisse en Wallonie depuis les années 2000. Cette baisse s'est d'ailleurs poursuivie après 2015, c'est-à-dire l'année prise en compte dans nos traitements $(14,7 \%$ en $2013 ; 12,9 \%$ en $2014 ; 13,1 \%$ en $2015 ; 10,3 \%$ en $2016 ; 10,5 \%$ en 2017) ${ }^{111}$. Les fortes fluctuations d'une année sur l'autre laissent toutefois présager que cette donnée, qui résulte d'enquêtes, n'est pas parfaitement robuste.

Carte 9. Taux d'abandon scolaire précoce (2015)

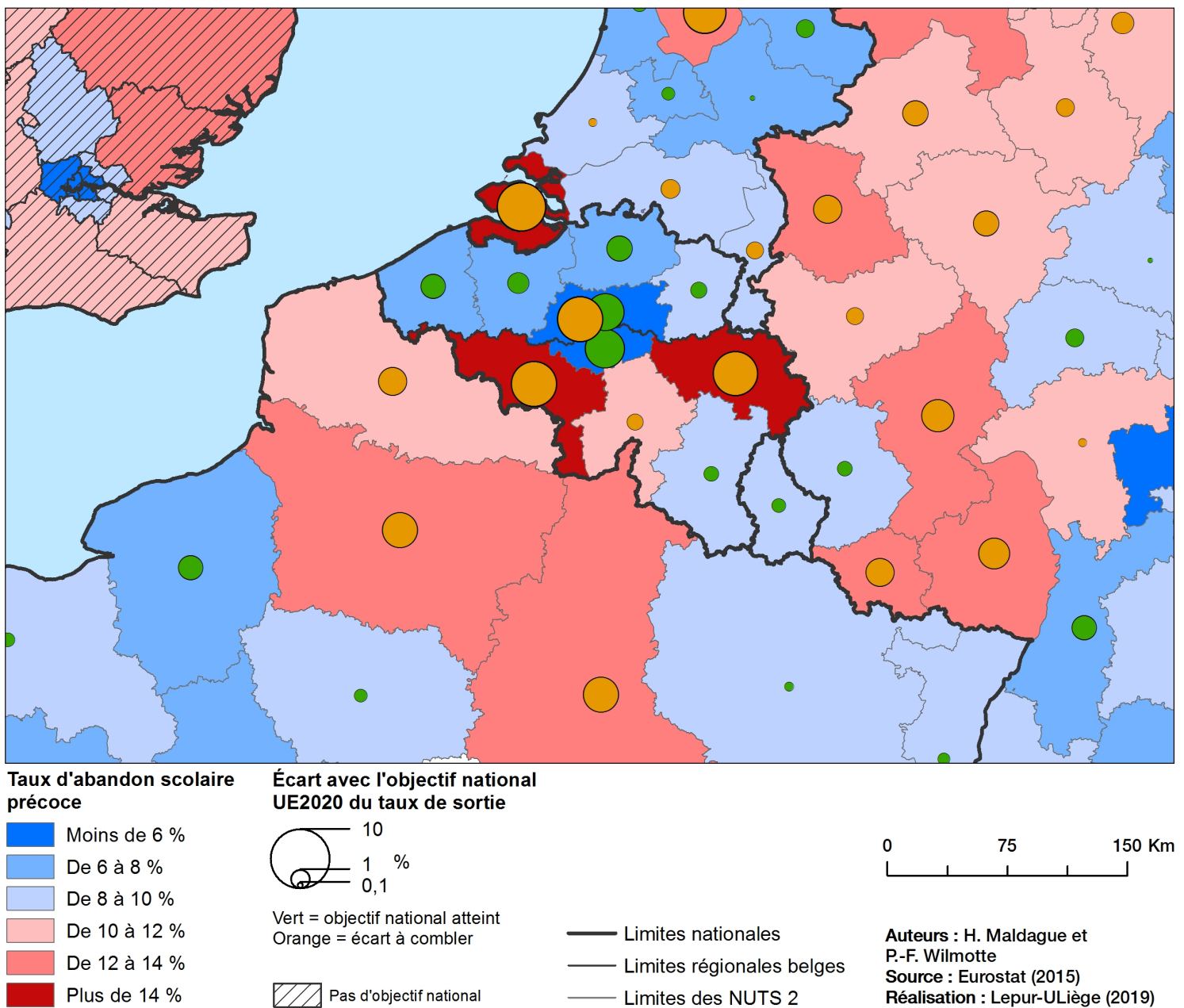

110 A. BAYE, «Inégalités entre élèves et inégalités structurelles en Fédération Wallonie-Bruxelles. L'apport de l'enquête PISA ", Éduquer: Tribune läque, nº 114, 2015, p. 10-12.

11 Source : Eurostat, «Early leavers from education and training by sex and NUTS 2 regions », 24 février 2020, http://appsso.eurostat.ec.europa.eu. 


\subsubsection{En synthèse sur les provinces wallonnes}

Pour l'année 2015, l'indice composite affiche une valeur de 95,4 pour le Brabant wallon. Cela correspond à un niveau de développement comparable aux provinces flamandes de Brabant flamand, de Flandre orientale et d'Anvers. Un tel niveau de développement est également comparable à celui des territoires européens les plus compétitifs, comme le Brabant septentrional, le Bade-Wurtemberg ou l'Île-de-France. La province de Brabant wallon a obtenu le score maximal pour les indicateurs de R\&D, de part de diplômés de l'enseignement supérieur et de taux de sortie précoce du système scolaire. Par contre, l'objectif affiché de 73,2\% de taux d'emploi n'y a pas (encore) été atteint.

La province de Namur atteint un objectif sur les quatre pris en compte, celui relatif à la part de diplômés de l'enseignement supérieur. Son résultat global est de 78,3 points, ce qui lui permet d'occuper la deuxième place parmi les provinces wallonnes. Le résultat global atteint par la province de Namur est toutefois pénalisé par le faible investissement en $\mathrm{R} \& \mathrm{D}$, un critère où ce territoire affiche des valeurs comparables à des territoires très ruraux.

La province de Liège affiche un indice composite de 74,6 points. À ce stade, ce territoire n'a atteint aucun des objectifs Europe 2020, bien que son positionnement en R\&D soit relativement bon en regard des régions environnantes. Les points forts de cette province correspondent à la part de diplômés de l'enseignement supérieur et à l'investissement en R\&D. Pour autant, un taux d'emploi trop faible et des départs précoces du système scolaire trop fréquents pénalisent fortement la moyenne globale. Au final, le profil de la province de Liège apparaît assez proche de celui de la région de Bruxelles-Capitale.

La province de Luxembourg se caractérise par une situation contrastée. D'un côté, l'objectif européen est atteint pour le taux de sortie précoce du système scolaire puisque le résultat est sous le seuil des 9,5\%. Par contre, d'un autre côté, la part du PIB investie en R\&D est l'une des plus faibles en Europe (le score spécifique est ici de 25,5/100). Il en résulte un indice composite de 70,4 points.

La province de Hainaut affiche l'indice composite le plus faible des cinq provinces wallonnes : 63,5 points. Cet indice est aussi l'un des plus faibles de la zone d'étude. En outre, le Hainaut a perdu 3,4 points entre 2010 et 2015. Face à cette situation, il n'est pas étonnant que certains observateurs s'interrogent sur la capacité de cette province à atteindre les objectifs européens ${ }^{112}$. Les résultats du Hainaut sont plus homogènes que les résultats de la province de Luxembourg, mais ses faiblesses les plus importantes se situent au niveau de la part de diplômés de l'enseignement supérieur et, surtout, du taux de sortie précoce du système scolaire.

La synthèse sur les provinces wallonnes illustre la pluralité de la Wallonie en matière de développement territorial et les importantes disparités socio-spatiales qui marquent la région. L'objectif des prochains chapitres est précisément d'affiner les contours de ces disparités socio-spatiales et d'analyser les différentiels de ressources territoriales qui en sont à l'origine.

112 R. GONZALEZ et al., SIESTA. Spatial Indicators for a "Europe 2020 Strategy" Territorial Analysis. Scientific report, Luxembourg, ESPON, 2012. 


\section{LA WALLONIE : UNE ÉCONOMIE PRODUCTIVE ET PRÉSENTIELLE}

Suite au cadrage réalisé à l'échelle de l'Europe du Nord-Ouest, nous pouvons préciser et compléter l'analyse par des traitements à l'échelle de la Wallonie. Pour ce faire, nous allons d'abord nous intéresser aux disparités socio-spatiales intra-wallonnes en termes d'économie productive et d'économie présentielle.

Des sources diverses ont été utilisées pour les analyses de ce chapitre. L'une d'elles correspond aux données décentralisées de l'Office national de sécurité sociale (ONSS) de 2015 sur les emplois salariés au lieu de travail. En complément, nous avons également bénéficié de la base de données Leodica, développée dans le cadre d'une collaboration entre l'intercommunale de développement économique de la province de Liège (la SPI) et Didier Van Caillie (HEC Liège - École de gestion de l'ULiège). Cette base de données contient les principales variables comptables des entreprises dont le siège social est situé en Wallonie et qui disposent de minimum 1000 euros d'actifs. Pour 2016, cela représente environ 100000 entreprises. La base de données a été développée à partir de données fournies par l'outil Belfirst du Bureau Van Dijk, sur la base des informations recensées par la Centrale des bilans de la Banque nationale de Belgique (BNB).

Les données relatives à l'emploi, qu'elles proviennent de l'ONSS ou de Leodica, sont ventilées par commune. Pour l'ONSS, nous avons utilisé les statistiques décentralisées qui sont établies au niveau des établissements. Par contre, Leodica ne renseigne les données qu'au niveau du siège social, ce qui limite son utilité pour les firmes multi-établissements. Pour les deux sources, nous disposons de l'information par secteur NACE ${ }^{113}$ à deux chiffres. Cela représente environ une centaine de secteurs économiques.

Grâce à cette ventilation par secteur, nous avons pu classer les emplois afin d'approcher la typologie des flux de revenus présentée au tableau 1. Trois catégories ont ainsi été définies: les emplois de la base productive pour approcher le type "fabrication", les emplois marchands de l'économie présentielle pour approcher le type «tourismenavettes » et les emplois non marchands de l'économie présentielle pour approcher le type "services locaux ${ }^{114}$.

113 La Nomenclature statistique des activités économiques dans la Communauté européenne (NACE) est un système de classification des activités économiques.

114 Les secteurs de l'agriculture et des industries extractives n'ont pas été traités en raison des ressources territoriales spécifiques sur lesquelles ils reposent (la qualité des sols et des sous-sols). Pour autant, les activités productives qui en découlent ont été prises en compte via le type «fabrication». 
Pour la base productive, ce sont les secteurs suivants qui ont été pris en compte : le secteur agroalimentaire (NACE 10 et 11); la fabrication de produits à base de tabac (NACE 12); le secteur textile (NACE 13 à 15); la pharmacie (NACE 21); le secteur chimique (NACE 19, 20 et 22) ; le secteur de la métallurgie (NACE 24) et des produits métalliques, hors machines et équipements (NACE 25) ; la fabrication et construction de machines-outils, de véhicules et de matériel de transport (NACE 26 à 30); l'artisanat et la fabrication de meubles (NACE 16 et 31 ); les autres industries manufacturières (NACE 17, 18, 23 et 32) (papier, carton, imprimerie, autres productions) ; la production, le traitement et le stockage des eaux, de l'électricité, de gaz, de vapeur et d'air conditionné (NACE 35 et 36); le transport et la logistique (NACE 49 à 53); les services d'information et de communication (NACE 58 à 63); les services financiers (NACE 64 à 66); les autres services aux entreprises (knowledge-intensive business services - KIBS) (NACE 69 à 75).

Pour les emplois marchands de l'économie présentielle, notre sélection s'est portée sur les secteurs suivants : les activités économiques du recyclage (NACE 33, 37, 38, 39, 95) (collecte des déchets, réparation et installation des machines et équipements, épuration des eaux usées, dépollution et réparation d'ordinateurs et de biens domestiques) ; les commerces et services de proximité, y compris l'horeca (NACE 45 à 47, 55, 56, 95, 96, 97, 98) ; les loisirs récréatifs dont les jeux de hasard (NACE 92 et 93) ; les services aux personnes (NACE 96 à 98); le commerce de gros et de détail (NACE 45 à 47); le soutien aux personnes et aux entreprises (NACE 77 à 82); la construction (NACE 41 à 43).

Enfin, ce sont les secteurs suivants qui ont été considérés pour quantifier les emplois non marchands de l'économie présentielle : le secteur associatif (NACE 94); l'administration publique et de défense, sécurité sociale obligatoire (NACE 84); l'enseignement (libre) (NACE 85); les services sociaux et de santé (NACE 86 à 88); les activités culturelles et de loisirs non marchands (NACE 90 et 91).

La suite de ce chapitre est structurée en trois sections. La première et la deuxième sections sont consacrées à la prise en compte d'indicateurs portant sur l'économie productive et sur l'économie présentielle. En troisième section, nous clôturons l'analyse par la présentation d'une typologie des communes en fonction de leurs profils productifs et/ou résidentiels.

\subsection{LA WALLONIE : UNE ÉCONOMIE PRODUCTIVE}

Notre analyse dédiée à l'économie productive aborde les quatre thématiques suivantes : les grandes entreprises de la base productive, l'emploi de la base productive, l'emploi dans les secteurs éligibles selon le Programme opérationnel FEDER 2014-2020 et la géographie des membres des pôles de compétitivité.

\subsubsection{Les grandes entreprises de la base productive}

Par leur importance dans le tissu économique, les grandes entreprises structurent les territoires où elles sont implantées, tant en rémunérant leurs salariés qu'en développant 
des partenariats scientifiques ou en entraînant les réseaux de fournisseurs dans leur sillage. Nous avons identifié les grandes entreprises sur la base d'une définition communément admise par l'administration fédérale et par l'administration wallonne ${ }^{115}$. Selon cette définition, une grande entreprise est une entreprise avec, soit plus de $250 \mathrm{ETP}$, soit un chiffre d'affaires de plus de 50 millions d'euros par an, soit un bilan annuel supérieur à 43 millions d'euros.

Afin de clarifier la thématique des grandes entreprises et la manière dont elles continuent de structurer l'économie wallonne, nous nous sommes appuyés sur Leodica. Pour rappel, Leodica rend compte des volumes d'emplois au siège social plutôt qu'à l'échelle des établissements, ce qui entraîne un biais pour les firmes multi-établissements ${ }^{116}$. Pour l'année 2016, Leodica recense ainsi 113 grandes entreprises disposant d'un siège social en Wallonie. Parmi ces 113 grandes entreprises, 75 appartiennent aux secteurs de la base productive telle que définie ci-dessus.

En 2005, sur la base de sources diverses, le consultant McKinsey a cherché à quantifier les volumes d'emplois des cinquante plus grandes entreprises privées actives en Wallonie ${ }^{117}$. Parmi ces cinquante entreprises, les quatre premières du classement étaient Arcelor (7 790 emplois), Caterpillar (3 630 emplois), GSK (3 500 emplois) et Duferco (3 190 emplois). En quinze ans, le tissu des grandes entreprises wallonnes a bien changé. Force est ici de constater le déclin de ce qu'il restait alors des grandes implantations industrielles. À l'heure où nous écrivons ces lignes, Caterpillar est en cours de liquidation et tant Arcelor (devenu ArcelorMittal) que Duferco (dont la majorité des activités ont été reprises par NLMK) ont subi de lourdes restructurations. Nous estimons que les emplois offerts par ces groupes représentent désormais moins de $20 \%$ des emplois qu'ils offraient en 2005.

L'analyse que nous venons de présenter sur les plus grands employeurs privés de la base économique wallonne n'a pu s'appuyer que sur des chiffres approximatifs. La cause première de cette situation tient au fait que le bilan d'une entreprise s'établit au siège social de chaque entité juridique. En conséquence, nous ne pouvons pas réellement rendre compte du poids d'une entreprise sur son territoire lorsque le siège d'exploitation ne correspond pas au siège social. En outre, les analyses longitudinales sont peu évidentes car les structures juridiques peuvent évoluer. Au final, force est de constater que les informations sur les grandes entreprises de la base productive ne sont pas suffisamment robustes. Face à leur rôle structurant, il s'agit là d'un manque criant du point de vue de la maîtrise de l'information économique.

Passer en revue la liste des 75 entreprises de la base productive illustre les mutations structurelles qui ont marqué l'économie wallonne durant les dernières décennies. Désormais, les plus grandes entreprises de Wallonie n'appartiennent plus aux secteurs économiques caractéristiques de la révolution industrielle (la métallurgie) ou du fordisme (la fabrication de machines-outils). Ils relèvent plutôt des domaines sélectionnés pour

115 SPW, Direction générale de l'Économie et de l’Emploi, « Notice du dossier simplifié préalable à la demande d'intervention ", s.d., http://forms6.wallonie.be.

116 Ce biais tend à se résorber suite à la sixième réforme de l'État. En effet, la régionalisation croissante des politiques économiques et d'emploi a, depuis 2014, forcé les entreprises à déclarer les emplois par région administrative. Une entreprise multi-établissements active dans les trois régions belges doit désormais déclarer le nombre d'emplois par établissement pour associer l'emploi à une région, là où, auparavant, cette information n'était pas nécessaire.

117 McKinsey, S'inspirer des succès d'autres régions pour relancer la création d'activités en Wallonie (rapport final), Namur, Ministère de la Région wallonne, 2005, non publié. 
structurer les pôles de compétitivité et, en particulier, de la pharmacie. Par ailleurs, l'évolution de la hiérarchie des entreprises a changé : de quelques grandes structures avec plusieurs milliers de travailleurs, les grandes entreprises actuelles représentent plutôt des ensembles où l'on trouve de 250 à 1500 travailleurs. GSK fait exception à cette situation, avec un volume d'emplois qui dépasse désormais les 8000 unités.

\subsubsection{L'emploi de la base productive}

À l'échelle régionale, les secteurs intégrés dans la base productive représentent 234429 emplois ${ }^{118}$. Cela représente $\pm 23 \%$ du nombre total de travailleurs salariés. La carte 10 permet d'analyser la géographie de cet emploi productif. Nous y avons représenté le volume absolu d'emplois au lieu du travail ainsi que le ratio entre ce volume et la population active communale. Par population active communale, nous entendons les personnes qui résident dans la commune considérée et qui, soit travaillent (dans la commune ou ailleurs) (population active occupée), soit sont sans emploi et à la recherche d'un emploi (population active au chômage) ${ }^{119}$. Cela conduit à un indicateur qui exprime le nombre de salariés pour 1000 actifs. Pour l'échelle régionale, cet indicateur est de $\pm 10 \%$ (234 429 emplois dans la base productive pour 2314282 actifs).

Il ressort de la carte 10 que la géographie des emplois productifs est désormais largement déterminée par la géographie des parcs d'activités. Il ressort aussi de la carte 10 que l'essentiel des communes wallonnes affichent une part d'emplois productifs très faible et que ces emplois de la base productive sont très concentrés dans les communes urbaines.

Deux concentrations principales de communes à fort volume d'emplois productifs se dégagent. D’une part, le Triangle wallon, particulièrement visible sur cette carte puisque les emplois productifs sont essentiellement localisés dans des parcs d'activités situés le long des corridors autoroutiers joignant les différentes villes de cet espace. La seconde zone de concentration correspond au bassin de Liège, où la densité d'emplois productifs est la plus forte de Wallonie, à partir d'une structure monocentrique centrée sur Liège.

Au-delà de ces deux concentrations, la Wallonie picarde et l'Entre-Vesdre-et-Meuse tirent leur épingle du jeu, en s'appuyant sur leur chapelet de villes petites et moyennes. En dehors de ces espaces, les petites villes situées au sud du sillon wallon représentent des volumes plus faibles. Toutefois, le ratio entre le volume d'emplois productifs et la population active communale est élevé dans quelques communes correspondant à des petites villes, avec l'effet remarquable de L'Oréal à Libramont ou de Burgo Ardennes à Virton. On trouve également une orientation productive à Marche-en-Famenne, ainsi qu'à Saint-Vith et à Vielsalm. À l'opposé, plusieurs communes des banlieues de tradition industrielle n'offrent presque plus d'emplois dans les domaines productifs. Cela concerne le Borinage, les communes de la région du Centre (hormis La Louvière et Manage), ainsi que certaines communes du bassin liégeois sans terrain portuaire ni parc d'activités de taille significative (Saint-Nicolas, Beyne-Heusay et Trooz).

118 D’après les données de l'ONSS en 2015.

19 Pour la définition officielle de la population active : «Population active, taux d'activité, taux d'emploi et taux de chômage administratifs par commune », IWEPS, www.iweps.be. 
Carte 10. Emploi de la base productive (2016)

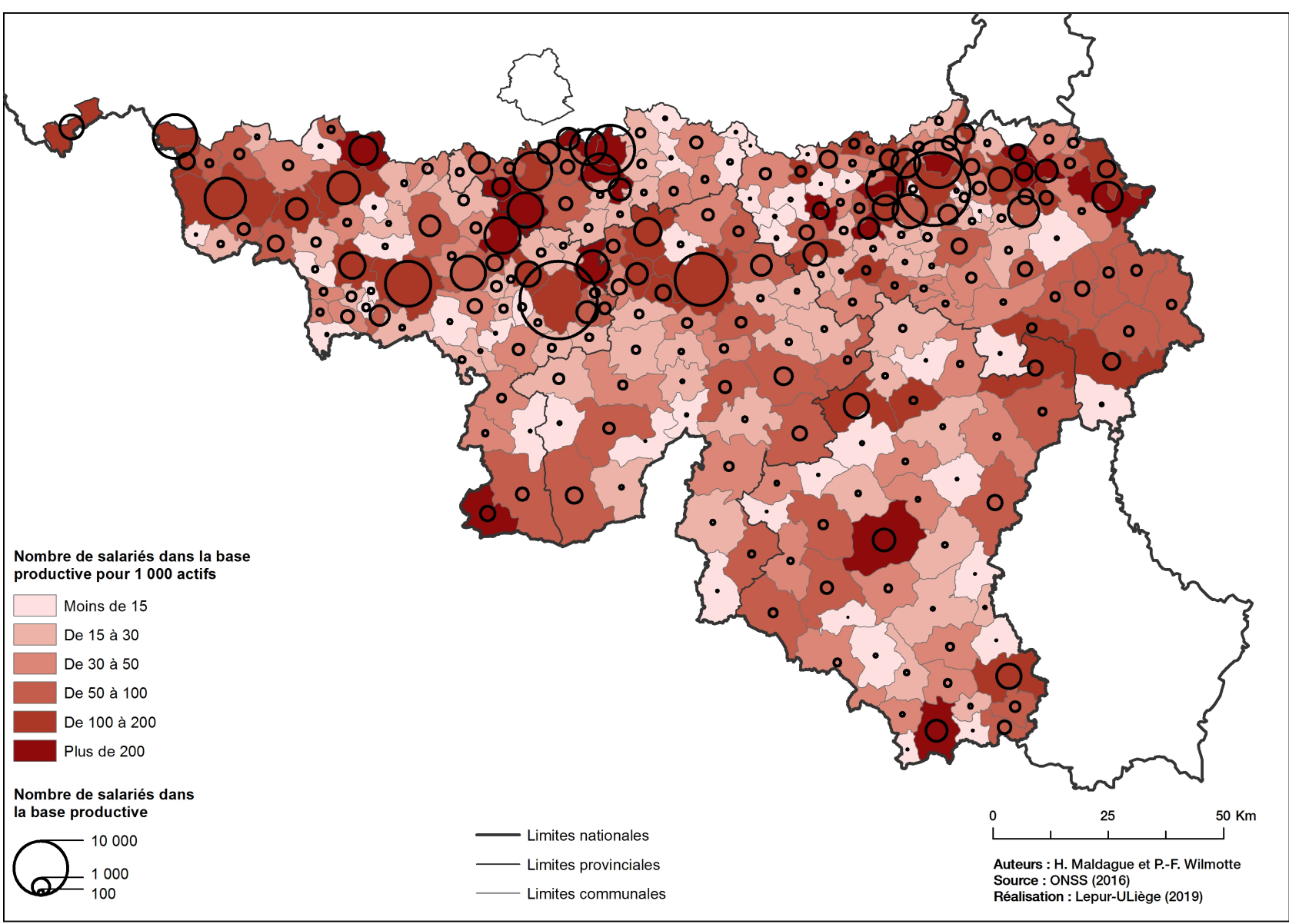

\subsubsection{L'emploi dans les secteurs éligibles selon le Programme opérationnel FEDER 2014-2020}

Nous avons relevé au chapitre 2 que le Programme opérationnel FEDER 2014-2020 prévoit des aides aux investissements pour les entreprises de certains secteurs. Les secteurs sélectionnés pour ces aides correspondent globalement aux secteurs de la base productive. Pour rappel, il s'agit des secteurs des clusters et des pôles de compétitivité, du tourisme, des services aux entreprises et de la R\&D. Pour rappel également, ces aides sont majorées dans deux cas : selon la taille (les PME sont privilégiées) et selon la localisation (cf. Carte 1).

Nous avons réalisé la carte 11 afin d'analyser la géographie des secteurs éligibles. Cette carte représente un indicateur établi grâce à la base de données Leodica (données de 2016). Il s'agit, par commune, du nombre d'entreprises éligibles pour 1000 actifs. Cette carte montre que, à l'échelle régionale, les secteurs éligibles sont peu implantés dans la plupart des communes hennuyères (à l'exception notable de la Wallonie picarde), dans la plupart des communes frontalières à la France ainsi que dans le Sud-Luxembourg. Les secteurs éligibles étant des secteurs porteurs, ce constat est à relier à la santé économique défaillante 
de ces territoires. À l'opposé, l'orientation sectorielle est bien plus favorable pour le Brabant wallon et, dans une moindre mesure, pour l'arrondissement de Verviers (à l'exception du bassin de Verviers et des communes sous orbite d'Aix-la-Chapelle).

À côté de ces disparités régionales, des disparités plus locales existent au sein des régions urbaines, notamment de Namur, de Liège et de Charleroi. Dans ces régions urbaines, les secteurs éligibles sont peu représentés tant dans les communes de tradition industrielle que dans les communes centrales, qui concentrent d'autres types d'activités comme le commerce de détail ou le non-marchand. Par contre, la spécialisation des communes résidentielles est plus favorable, en particulier lorsqu'elles accueillent un parc d'activités.

Carte 11. Entreprises éligibles pour les aides à l'investissement selon le Programme opérationnel FEDER 2014-2020 (2016)

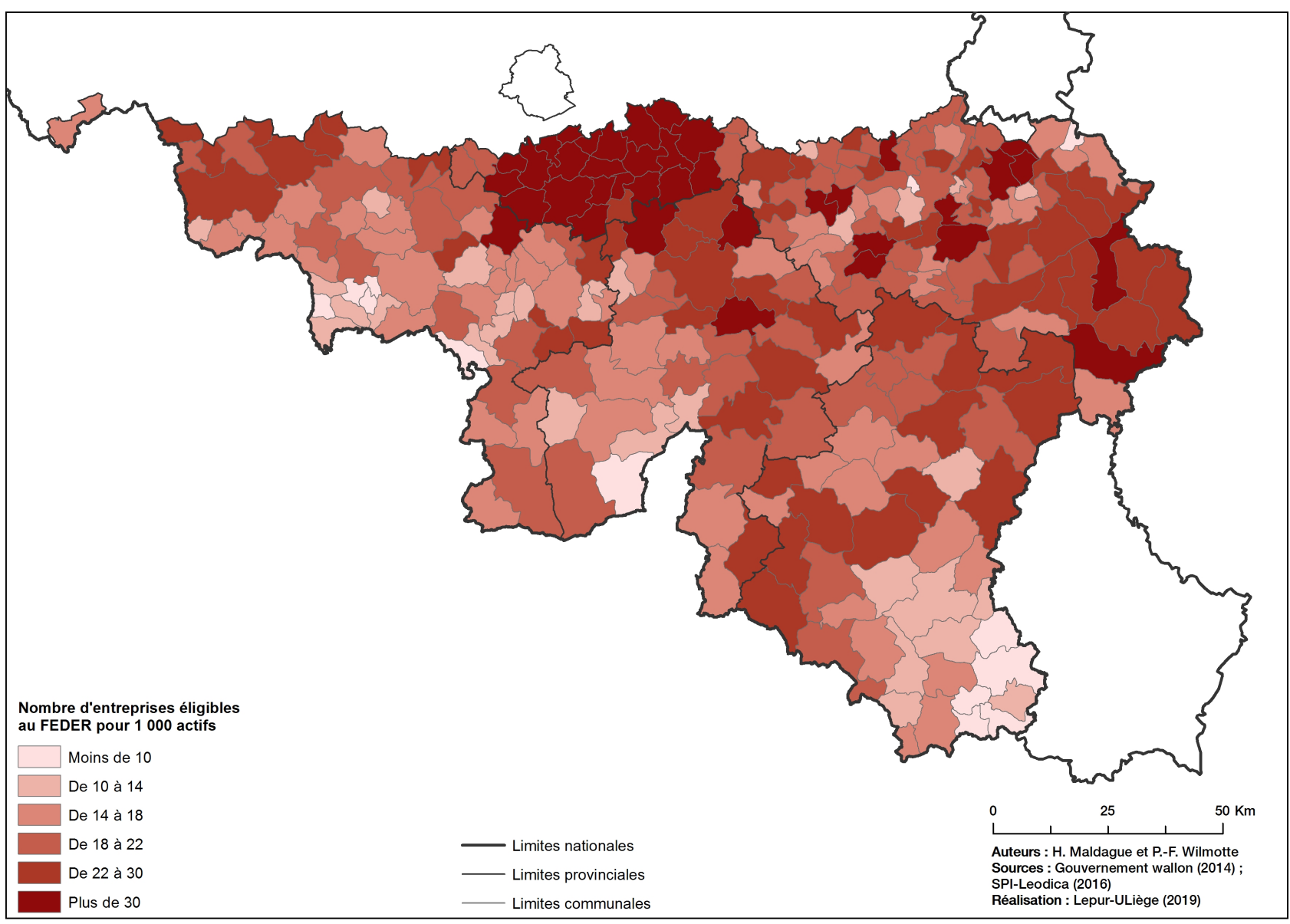

Afin d'affiner le diagnostic, il est illustratif de s'intéresser à la comparaison entre la ville de Mons et les deux pôles brabançons de Wavre et de Waterloo. En volumes absolus, le nombre total d'entreprises disposant de plus de 1000 euros d'actifs (tous secteurs confondus) est de l'ordre de 2400 à Mons et de l'ordre de 2200 tant à Wavre qu'à Waterloo. Ces chiffres témoignent d'une dynamique entrepreneuriale bien plus importante à Wavre et à Waterloo puisque ces localités sont trois fois moins peuplées que le chef-lieu du Hainaut $( \pm 95000$ habitants à Mons contre \pm 32000 habitants à Waterloo et \pm 30000 
à Wavre). S'agissant maintenant des entreprises éligibles, nous en trouvons \pm 1050 à Mons contre \pm 1100 à Wavre et à Waterloo. Au-delà de la dynamique entrepreneuriale, nettement plus forte à Wavre et à Waterloo, ces chiffres montrent aussi que les pôles brabançons bénéficient d'une orientation sectorielle plus favorable au regard des critères du programme FEDER 2014-2020.

Il est interpellant de confronter la géographie mise en avant par la carte 11 à la géographie prioritaire des aides (cf. Carte 1 et supra, 2.1.3.2). Cette mise en perspective montre que le gouvernement wallon a fait le choix de soutenir des territoires où le potentiel de la base productive est plutôt faible, comme la province de Hainaut ou comme les communes de tradition industrielle du bassin liégeois. Nous observons toutefois quelques exceptions à cette règle, avec des territoires à la fois dynamiques et prioritairement soutenus, parmi lesquels certaines communes hennuyères spécialisées dans les secteurs éligibles (en Wallonie picarde, dans la périphérie sud de Charleroi ou à proximité du Brabant wallon). D'autres exceptions correspondent aux communes brabançonnes de Nivelles et d'Ittre, reprises en zone 2, ainsi qu'à différentes communes de la province de Liège qui, comme nous l'avons analysé ci-dessus, semblent avoir été intégrées en zone 2 en raison de la présence de parcs d'activités sur leur territoire.

$\mathrm{Au}$ final, nous pouvons retenir que les aides FEDER aux entreprises ne sont pas focalisées sur les territoires où le vivier est le plus concentré et/ou spécialisé, mais plutôt sur les territoires en déclin. Ce choix peut se justifier en termes d'équité territoriale puisqu'il contribue à rééquilibrer le territoire en favorisant des espaces ou des parties d'agglomération accueillant relativement peu d'entreprises. Pourtant, vis-à-vis du programme opérationnel, cette orientation peut paraître paradoxale. En effet, d'un côté, le programme opérationnel met en exergue le renforcement des économies d'agglomération des plus grands pôles wallons et, d'un autre côté, la géographie des zones éligibles pénalise le Brabant wallon qui est le lieu où ces économies sont les plus développées. Comme nous allons le voir au point suivant, la logique - implicite plutôt qu'explicite - suivie par les Plans Marshall successifs est tout autre. En effet, les moyens destinés aux membres des pôles de compétitivité sont fortement concentrés dans les parties de la Wallonie les mieux irriguées par les économies d’agglomération.

\subsubsection{Les membres des pôles de compétitivité}

Pour compléter l'analyse sur l'économie productive, intéressons-nous maintenant aux pôles de compétitivité et à la géographie de leurs membres. Ce focus se justifie par le fait que les secteurs retenus pour les pôles rassemblent les plus grandes entreprises de la base productive. Une deuxième raison qui justifie le focus sur les membres des pôles est que les entreprises concernées sont plus performantes et disposent d'une croissance d'emplois et de valeurs ajoutées plus forte que la moyenne des entreprises wallonnes ${ }^{120}$. Par la prise en compte de la géographie des membres des pôles de compétitivité, nous pouvons donc appréhender la géographie wallonne de l'innovation et anticiper ainsi l'évolution probable de sa géographie économique.

120 M5 (bureau d'études), Pôles de compétitivité. Approche quantitative, impacts en 2012, Grez-Doiceau, M5, 2014, non publié. 
Les données sur les membres des pôles de compétitivité nous ont été communiquées par l'administration régionale wallonne en 2014, lors d'une recherche antérieure ${ }^{121}$. Elles nous ont permis d'identifier les membres des six pôles grâce à une liste de 1091 organisations ayant été en ordre de cotisation annuelle auprès d'un pôle à une reprise au moins entre 2005 et 2013. Ces données ont été utilisées pour réaliser la carte 12 et le tableau 4 . Ce tableau décrit les localisations privilégiées adoptées par les membres de chacun des six pôles.

Précisons que les données mobilisées ne renseignent pas l'importance économique des membres (valeur ajoutée, emploi, etc.). Précisons aussi que ces membres ne correspondent pas uniquement à des entreprises puisque des organisations diverses peuvent s'affilier aux pôles : des grandes entreprises et des PME, mais aussi des laboratoires universitaires, des centres de recherche indépendants ou encore des administrations publiques. Précisons encore que, sur les 1091 membres recensés, 931 sont situés en Wallonie, 87 à Bruxelles, 45 en Flandre et 28 à l'étranger. Il n'est donc pas obligatoire d'être installé en Wallonie, ou même en Belgique, pour devenir membre d'un pôle de compétitivité.

Carte 12. Localisation des membres des six pôles de compétitivité (2013)

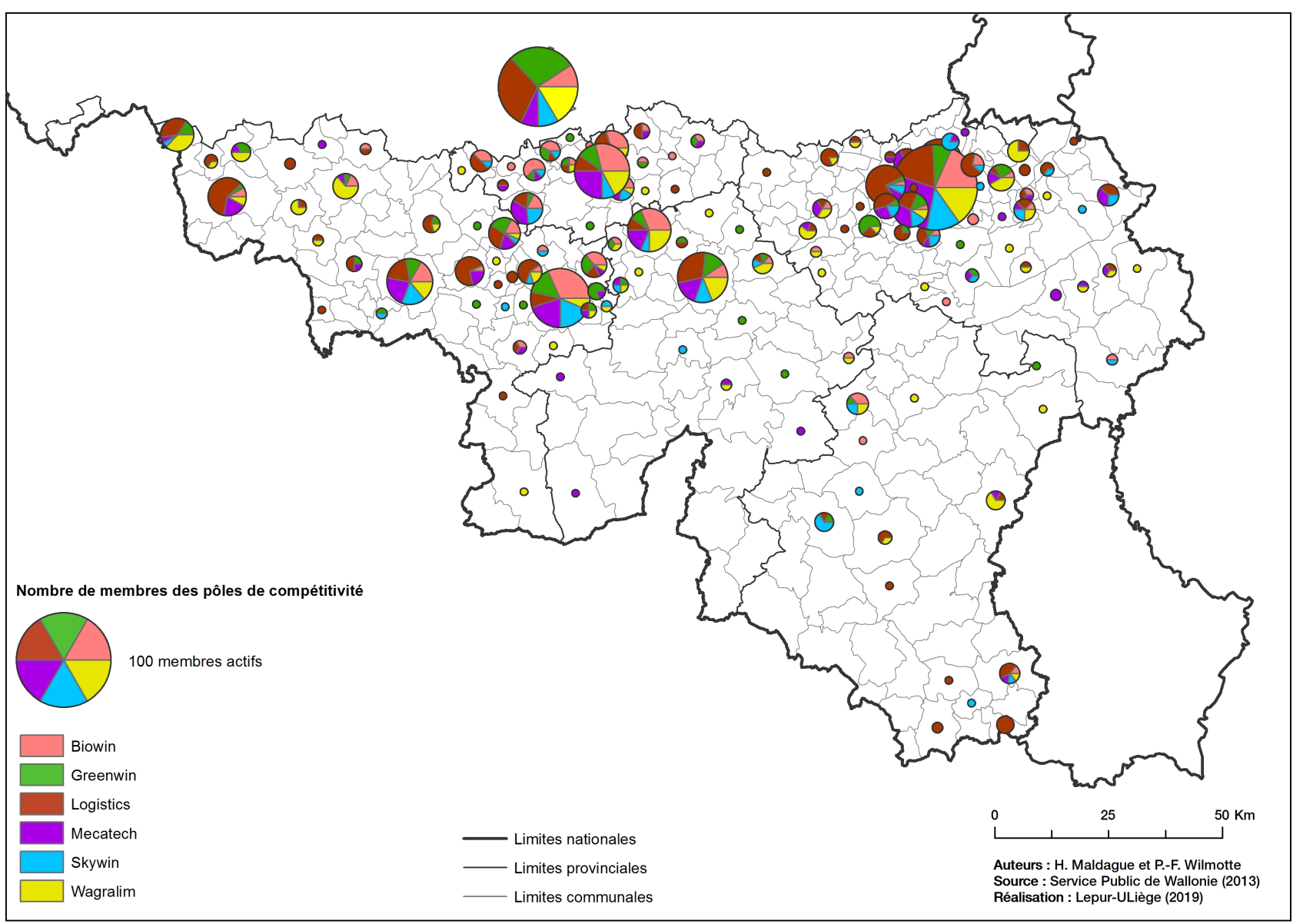

121 P.-F. Wilmotte, J.-M. HalleuX, « La structure spatiale des systèmes régionaux d'innovation », op. cit., p. $51-70$. 
Tableau 4. Localisations privilégiées des membres des six pôles de compétitivité

\begin{tabular}{|c|c|}
\hline Pôle de compétitivité & Principaux territoires d'implantation \\
\hline Biowin & $\begin{array}{l}\text { Le Triangle wallon est le principal territoire, avec Wavre-Ottignies-Louvain- } \\
\text { la-Neuve et l'aéropôle de Charleroi comme principaux centres. } \\
\text { La région liégeoise est un lieu de concentration de second ordre, principalement } \\
\text { sur le territoire de la ville de Liège (Liège Science Park ou centre-ville). } \\
\text { Nombre marginal de membres hors de ces territoires. } \\
\text { Importance des grands pôles structurants : l'essentiel des membres est concentré } \\
\text { dans cinq localités (Wavre, Ottignies-Louvain-la-Neuve, Liège, Gembloux } \\
\text { et Charleroi). }\end{array}$ \\
\hline Greenwin & $\begin{array}{l}\text { Le Triangle wallon concentre l'essentiel des membres du pôle avec une forte } \\
\text { prépondérance des membres situés à Bruxelles. } \\
\text { La région liégeoise accueille une concentration secondaire de membres. } \\
\text { Les membres sont essentiellement localisés dans les communes périphériques } \\
\text { des pôles urbains. }\end{array}$ \\
\hline Logistics in Wallonia & $\begin{array}{l}\text { La région liégeoise représente le premier territoire d'implantation des membres } \\
\text { du pôle logistique. } \\
\text { Persistance d'une concentration autour du bassin industriel traditionnel } \\
\text { du Hainaut mais aussi d'acteurs situés à et autour de Bruxelles dans une } \\
\text { moindre mesure. } \\
\text { Concentrations notables dans les territoires proches de Lille et Luxembourg, } \\
\text { dans le Brabant wallon et dans l'est de la province de Liège en fonction de la } \\
\text { localisation des parcs d'activités. } \\
\text { La localisation des membres dépend essentiellement de la localisation des parcs } \\
\text { d'activités (localisation essentiellement plus périphérique) et des sièges sociaux } \\
\text { des membres impliqués dans le pôle. }\end{array}$ \\
\hline Mecatech & $\begin{array}{l}\text { La région liégeoise (monocentrique) et le Triangle wallon (polycentrique avec } \\
\text { Charleroi et Ottignies-Louvain-la-Neuve) sont deux concentrations d'importance } \\
\text { semblable en termes de membres. } \\
\text { Au-delà de ces deux territoires, quelques membres sont essentiellement localisés } \\
\text { en Wallonie picarde et dans l'est de la province de Liège. } \\
\text { Les membres sont essentiellement localisés dans les communes centrales } \\
\text { des pôles urbains. }\end{array}$ \\
\hline Skywin & $\begin{array}{l}\text { La région liégeoise est le principal pôle de l'aéronautique et du spatial en Wallonie. } \\
\text { Le Triangle wallon se dégage ici aussi avec un nombre de membres (des membres } \\
\text { importants) à Charleroi. } \\
\text { Transinne (Libin) accueille quelques membres du pôle Skywin (centre Galaxia), } \\
\text { mais cela ne semble pas générer une masse critique suffisante pour induire } \\
\text { des effets d'agglomération. } \\
\text { Deux types de localisation émergent : d'une part, des sites installés en parc } \\
\text { d'activités et, d'autre part, des institutions, sièges sociaux ou des sociétés } \\
\text { de services aux entreprises du secteur localisés dans les communes centrales } \\
\text { des pôles urbains. }\end{array}$ \\
\hline Wagralim & $\begin{array}{l}\text { Diffusion des membres, essentiellement, au nord du sillon Sambre-et-Meuse } \\
\text { avec des concentrations notables dans les villes universitaires (Liège, Gembloux, } \\
\text { Louvain-la-Neuve) ainsi que dans les petites villes du Pays de Herve et de } \\
\text { la Wallonie picarde où une tradition agro-alimentaire est historiquement } \\
\text { implantée. } \\
\text { Forte diffusion des localisations où les institutions et les sièges sociaux occupent } \\
\text { les centres-villes des principaux pôles urbains tandis que les activités de production } \\
\text { sont plutôt situées dans des parcs d'activités ou dans des petites villes. }\end{array}$ \\
\hline
\end{tabular}

Source : synthèse provenant de P.-F. WiLmOTTE, J.-M. HALLEUX, « La structure spatiale des systèmes régionaux d'innovation : qu'en est-il de la proximité géographique au sein des pôles de compétitivité ? », L'Espace géographique, volume 47, $\mathrm{n}^{\circ} 1,2018$.

La géographie des membres des pôles de compétitivité wallons est très semblable à la géographie de l'emploi salarié dans les secteurs dits productifs. Comme pour les emplois productifs, le Triangle wallon et la région liégeoise sont les deux concentrations majeures. 
Cette situation est à analyser à travers la grille d'analyse des économies d'agglomération puisque ces parties de la Wallonie en sont les mieux dotées (cf. Tableau 4). À la différence des aides FEDER, les moyens mobilisés par les pôles de compétitivité tendent donc à privilégier les parties de la Wallonie où l'économie productive est la plus active.

Il se dégage de la carte 12 que la Wallonie picarde et le nord-est de la province de Liège parviennent à se positionner dans les pôles Logistics in Wallonia et Wagralim. Cela s'explique parce que, en comparaison des quatre autres pôles, les économies d'agglomération sont moins vitales dans la logistique et dans l'agro-alimentaire. Par ailleurs, la représentation significative de la Wallonie picarde et du nord-est de la province de Liège dans les pôles Logistics in Wallonia et Wagralim est cohérente avec les spécialisations économiques de ces territoires. En dehors des zones de concentration que nous venons de décrire, les autres parties de la Wallonie sont très peu représentées au sein des pôles de compétitivité. On trouve juste quelques entreprises isolées, une petite concentration en logistique dans la région d'Arlon et l'implantation de Galaxia à Redu. Ce résultat pousse à s'interroger sur les modalités qui permettraient de développer l'économie de la connaissance dans ces parties de la Wallonie peu dotées en économies d'agglomération.

\subsection{LA WALLONIE : UNE ÉCONOMIE PRÉSENTIELLE}

Trois sous-sections vont maintenant être développées afin de traiter la question de l'économie présentielle. Ce sera là l'occasion de faire le point, primo, sur les revenus moyens et médians des résidents des communes wallonnes; secundo, sur les emplois dans les secteurs du présentiel ; tertio, sur la géographie du chômage.

\subsubsection{Les revenus des résidents}

$\mathrm{Vu}$ la disjonction croissante entre les lieux de production et les lieux de consommation, la géographie des revenus est de plus en plus étrangère à la géographie des emplois productifs ${ }^{122}$. Afin d'analyser cette géographie des revenus, nous avons élaboré deux cartes : la carte 13 sur les revenus moyens par habitant et la carte 14 sur les revenus médians par déclaration.

122 D. Guex, O. Crevoisier, « Globalisation postindustrielle et milieux locaux : une typologie », op. cit.; R. CAMAGNI, «Attractivité et compétitivité, un binôme à repenser», op. cit., p. 11-15. 
Carte 13. Revenus moyens par habitant (2016)

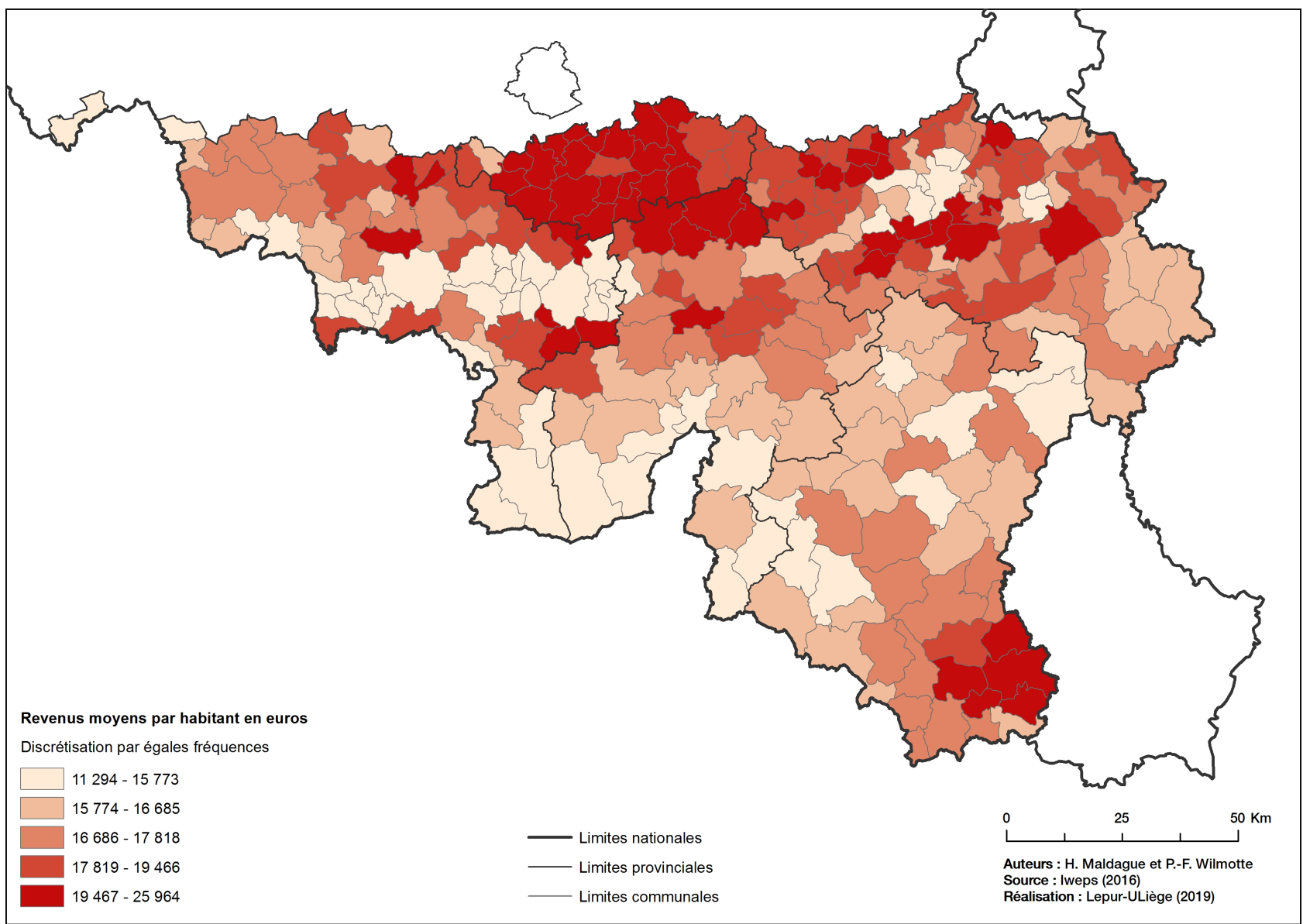

Les hauts revenus moyens par habitant sont l'apanage des banlieues périurbaines et notamment du Brabant wallon, qui bénéficie à la fois de son économie productive et de la redistribution du potentiel économique bruxellois. Cette zone de hauts revenus moyens centrée sur le Brabant wallon se prolonge dans le nord du Hainaut mais surtout dans le nord de la province de Namur. Ailleurs en Wallonie, de hauts revenus moyens sont observés dans le Sud-Luxembourg, à proximité du Grand-Duché et de Luxembourg-Ville en particulier. De hauts revenus moyens caractérisent aussi les communes d'ancienne périurbanisation. Cela concerne le sud des régions urbaines de Verviers (Jalhay), de Liège (depuis Olne jusque Tinlot), de Namur (Profondeville) et de Charleroi (Montigny-leTilleul, Ham-sur-Heure-Nalinnes et Gerpinnes).

Des revenus moyens faibles caractérisent deux types de localités. Il s'agit d'abord de communes rurales éloignées des pôles d'emplois, en particulier le long de la frontière française. Il s'agit ensuite de communes urbaines et des bassins de tradition industrielle (du Borinage à Verviers). L'extension des bas revenus moyens est particulièrement étendue dans le Hainaut, depuis le Borinage jusque Sambreville en province de Namur. Une situation comparable est observée dans le bassin de Liège, mais sur une moindre extension spatiale. Ces zones de faibles revenus moyens intègrent des communes de tradition industrielle devenues économiquement atones, mais aussi des communes centrales (Mons, La Louvière, Charleroi, Liège et Verviers) où, depuis maintenant plus d'un demi-siècle, l'environnement urbain dégradé a poussé les classes moyennes à s'exurbaniser. 
Sans surprise, la géographie des revenus médians présente des points communs avec la géographie des revenus moyens. Pour les zones à faibles revenus, on trouve les mêmes communes, à savoir les communes rurales proches de la France, les communes de tradition industrielle et les grandes villes. Pour les zones à hauts revenus, les deux géographies sont plus différenciées. En effet, les communes à hauts revenus moyens ne sont pas nécessairement des communes à hauts revenus médians. Cela concerne le Brabant wallon, qui n'apparait plus aussi nettement comme la zone la plus favorisée de Wallonie. Le même décalage touche également plusieurs communes d'ancienne périurbanisation des régions de Liège, Namur et Charleroi (en particulier Chaudfontaine, Neupré, Profondeville, Montigny-le-Tilleul et Gerpinnes).

Pour expliquer ce décalage entre hauts revenus moyens et médians, il faut faire référence au vieillissement. De nombreuses communes du Brabant wallon ainsi que les communes d'ancienne périurbanisation citées se sont massivement peuplées dans les années 1970 et 1980. En conséquence, on y trouve aujourd'hui une proportion importante de retraités dont les niveaux actuels de revenus sont limités. En parallèle, on y trouve aussi un immobilier souvent coûteux, auquel seuls des actifs aux revenus très élevés peuvent accéder. Or, statistiquement parlant, ces revenus très élevés ont moins d'impact sur l'indicateur du revenu médian que sur l'indicateur du revenu moyen.

Carte 14. Revenus médians par déclaration (2016)

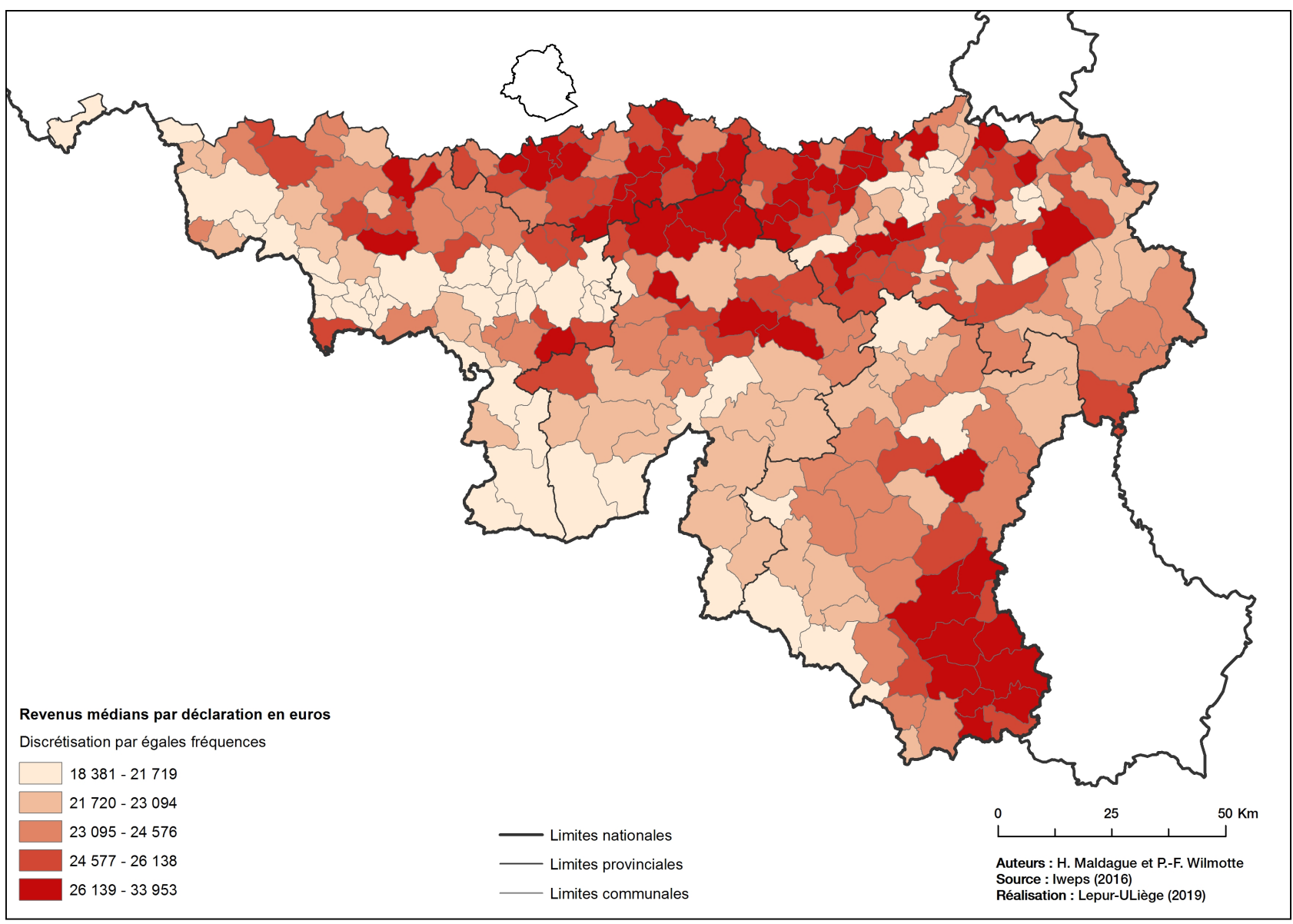


La comparaison entre les cartes 13 et 14 permet également d'identifier des communes aux revenus moyens limités et aux revenus médians élevés. Il s'agit généralement de communes tardivement périurbanisées. Elles se distinguent des premières couronnes périphériques par un marché du logement plus accessible et par une proportion moindre de retraités. On trouve ce profil de communes dans l'ouest de la province de Liège, où l'emploi productif est peu développé mais où les habitants bénéficient d'un cadre résidentiel attractif et de la proximité aux autoroutes vers Namur et Bruxelles. Cette proximité donne accès à plusieurs pôles d'emplois, depuis Liège jusque Bruxelles en passant par Namur et le Brabant wallon. La province de Luxembourg compte également de nombreuses communes aux revenus moyens limités et aux revenus médians élevés. L'influence grand-ducale y est ainsi notable sur un territoire plus vaste en termes de revenus médians (jusque Bertogne et Tenneville) qu'en termes de revenus moyens (jusque Habay).

\subsubsection{Les emplois dans les secteurs du présentiel}

La question des emplois dans les secteurs du présentiel est abordée ci-dessous par une analyse des géographies de la base présentielle marchande et de la base présentielle non marchande. En complément, nous développons également une réflexion sur les secteurs du tourisme et du commerce.

\subsubsection{La base présentielle marchande}

La carte 15 porte sur les emplois relevant des secteurs de la base présentielle marchande. Rappelons que les secteurs sélectionnés correspondent au commerce (de gros et de détail), à l'horeca, à la construction, aux activités liées au recyclage et au traitement des eaux, aux activités de loisirs récréatifs et aux services aux personnes (hors santé et social). À l'instar de la carte 10 sur les emplois de la base productive, nous y avons représenté le volume absolu d'emplois ainsi que le rapport entre ce volume et la population active.

Il ressort de la carte 15 que les grandes villes wallonnes concentrent des volumes d'emplois importants dans les secteurs du présentiel marchand. Ce constat est lié au fait que ces villes continuent de concentrer les pôles commerçants les plus importants.

En termes de spécialisation, on rencontre un grand nombre d'emplois par actif dans les pôles brabançons de Wavre et Nivelles, mais aussi dans plusieurs communes situées au sud du sillon Sambre-et-Meuse, particulièrement en Famenne et en Ardenne. La première explication est que ces communes du sud du sillon accueillent des centres de distribution de biens et de services de proximité pour les territoires ruraux alentour. Une seconde explication réside dans l'éloignement relatif de ces petites villes par rapport aux grands pôles urbains wallons, à la différence des villes de même taille situées au nord du sillon.

Le développement du tourisme explique aussi la spécialisation dans le présentiel marchand de certaines petites villes au sud du sillon. En raison vraisemblablement d'un tourisme moins pourvoyeur d'emplois, les villes de l'Entre-Sambre-et-Meuse s'y positionnent moins bien que les villes de la Famenne et de l'Ardenne. 
Carte 15. Emplois marchands de l'économie présentielle (2016)

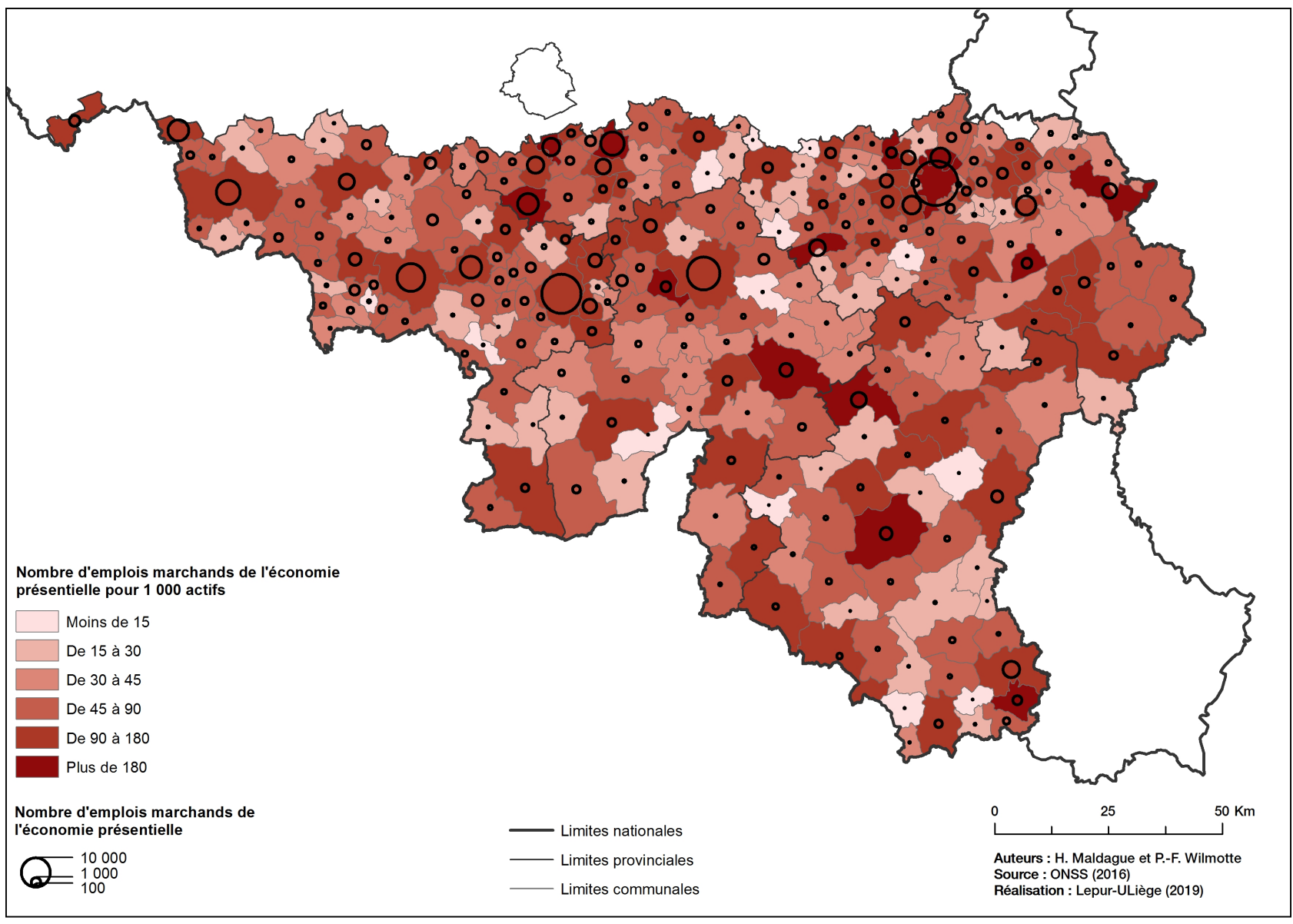

\subsubsection{La base présentielle marchande et les enjeux du tourisme et du commerce}

Malgré son poids économique important, estimé à environ $6 \%$ du PIB régional, le secteur du tourisme reste peu développé en Belgique en général et en Wallonie en particulier. En effet, si l'on retient les indicateurs du nombre de nuitées par habitant ou du nombre de lits par habitant, le secteur apparaît sous-développé vis-à-vis des pays voisins ${ }^{123}$.

Le tourisme wallon est classiquement organisé autour des deux piliers que sont les pôles urbains et l'Ardenne. L'Ardenne y dispose à la fois d'une spécialisation économique et d'une infrastructure importante héritée d'une mise en tourisme qui date du XIX ${ }^{\mathrm{e}}$ siècle ${ }^{124}$. Pour autant, l'analyse détaillée des nuitées sur les vingt dernières années met en exergue le déclin du tourisme de séjour en Ardenne, avec une chute de l'ordre de 10 à $20 \%$ en province de Luxembourg entre 2001 et 2015. Bien que les données disponibles soient

123 S. QUÉRIAT, «Secteur tourisme », in M.-F. GODART, Y. HANIN (dir), Recherche R1 : État du territoire wallon (dynamiques sectorielles territorialisées), Namur, CPDT, 2016, p. 49-59; J.-M. LAMBOTTE, X. DUPONT, H.-J. Gathon, H. Maldague, F. Durant, C. Lamour, N. Raimbault, A. Malherbe, M. Grandjean, A. LeCLERCQ, A. NiHOUl, Schéma de développement territorial de la Grande Région. Cahier thématique $\mathrm{n}^{\circ} 3$ : Développement économique, Grande Région / Interreg Grande Région, 2018.

124 S. QUÉRIAT, La mise en tourisme de l'Ardenne belge, 1850-1914: genèse et évolution d'un espace touristique. Processus, acteurs et territoires, Thèse de doctorat, ULB, 2010. 
sujettes à caution, en lien notamment avec le développement des hébergements alternatifs et de terroir, cette évolution est interpellante pour une province où le tourisme occupe une place importante. Dans le même temps, comme ailleurs en Europe, le tourisme urbain a crû et la ville de Liège est devenue l'une des premières destinations wallonnes ${ }^{125}$. Entre 2001 et 2015, le nombre de nuitées a doublé à Charleroi, mais ici vraisemblablement en raison du développement de son aéroport. Plus généralement, l'investissement public et privé dans le Hainaut a permis d'y doubler le nombre de nuitées durant la période prise en compte, bien que le nombre de nuitées par habitant reste six fois moins important que dans les pays voisins, avec approximativement 0,8 nuitée par habitant en Hainaut contre 6 nuitées par habitant aux Pays-Bas, en France ou au Danemark ${ }^{126}$.

En plus d'être pénalisée par un insuffisant développement du tourisme, la base présentielle marchande wallonne est également pénalisée par les importantes concurrences frontalières dans le domaine du commerce de détail. Selon l'organisation patronale Comeos, en 2016, les concurrences frontalières auraient coûté 16000 emplois à l'économie belge ${ }^{127}$. L'importance des concurrences frontalières résulte de causes multiples, parmi lesquelles certains différentiels de prix pratiqués par la grande distribution ${ }^{128}$.

La problématique des concurrences frontalières est également indissociable de la problématique de la faible vitalité de nombreuses polarités commerciales ${ }^{129}$, dont on sait par ailleurs qu'elle va s'amplifier avec l'expansion du commerce électronique. À propos de la faible vitalité de nombreuses polarités wallonnes, il nous semble indispensable de souligner le caractère contre-productif de la multiplication d'une offre périphérique qui, contrairement au discours de certains promoteurs, ne crée pas de nouveaux emplois. En réalité, plutôt que de contribuer à la création d'emplois, le développement de l'offre périphérique réduit la base présentielle marchande par son impact négatif sur l'attractivité urbaine.

Afin d'illustrer les articulations entre l'attractivité urbaine et la base présentielle marchande, faisons référence à une étude néerlandaise sur les flux commerciaux au sein de l'Euregio Meuse-Rhin ${ }^{130}$. Cette étude a démontré que Liège perd un potentiel commercial considérable en raison d'une évasion vers le Limbourg néerlandais, où Maëstricht est devenue une polarité particulièrement attractive ${ }^{131}$. Ã la source de l'attractivité de cette ville, on trouve une politique restrictive vis-à-vis de la périurbanisation de l'offre commerciale. Il en a résulté une concentration de l'offre en milieu urbain qui, conjuguée à une stratégie de long terme visant à améliorer la qualité des espaces publics, a fait de Maëstricht le premier pôle commerçant de l'Euregio Meuse-Rhin. Face à Maëstricht, nous trouvons Liège, qui demeure le premier pôle commerçant de Wallonie, mais dont

125 Pour l'année 2015, Liège se classe à la troisième place derrière Vielsalm et Durbuy. Suivent ensuite Charleroi, Froidchapelle, Spa et La Roche-en-Ardenne.

126 Bundesministerium für Verkehr und Digital Infrastruktur, «Raumbeobachtung Deutschland und angrezende regionen », Bonn / Berlin, MORO Praxis 11, 2017.

RTBF Info, 30 janvier 2018, www.rtbf.be.

128 J.-M. LAmbOtTE, G. DevilLET, « Le commerce », Notes de recherche de la CPDT, nº 21, 2011.

129 J.-P. GRIMMEAU, B. WAYENS, « Les causes de la disparition du petit commerce (1945-2015) », Courrier hebdomadaire, CRISP, n 2301-2302, 2016; G. DEVILlET, M. JASPARD, J. VAZQUEZ PARRAS, Atlas du commerce en Wallonie. Structures, dynamiques et comportements spatiaux des consommateurs, Liège, Presses universitaires de Liège, 2014.

${ }_{130}$ BRO, Grenzeloos winkelen 2009, Boxtel, Provincie Limburg, 2009.

131 Vis-à-vis des territoires de l'Euregio, la région liégeoise verrait une fuite commerciale de l'ordre de 80 millions d'euros de chiffre d'affaires en 2009. 
l'attractivité commerciale est moins importante que son potentiel démographique le laisserait attendre. La cause structurelle de ce déficit d'attractivité est bien connue. Elle correspond à la dispersion de l'offre en nouveaux points de vente induite, durant des décennies, par une gestion des implantations commerciales n'ayant pas assez tenu compte des critères d'aménagement du territoire ${ }^{132}$.

\subsubsection{La base présentielle non marchande}

La carte 16 vise à appréhender la géographie de la base présentielle non marchande. Elle a été établie à partir des emplois du secteur associatif, de l'enseignement, des services sociaux et de santé ainsi que des activités culturelles et de loisirs non marchands. La base présentielle non marchande est largement déterminée, d'une part, par le secteur des hôpitaux et des intercommunales de soins et, d'autre part, par les secteurs de l'administration, de l'enseignement et de la culture.

Carte 16. Emplois non marchands de l'économie présentielle (2016)

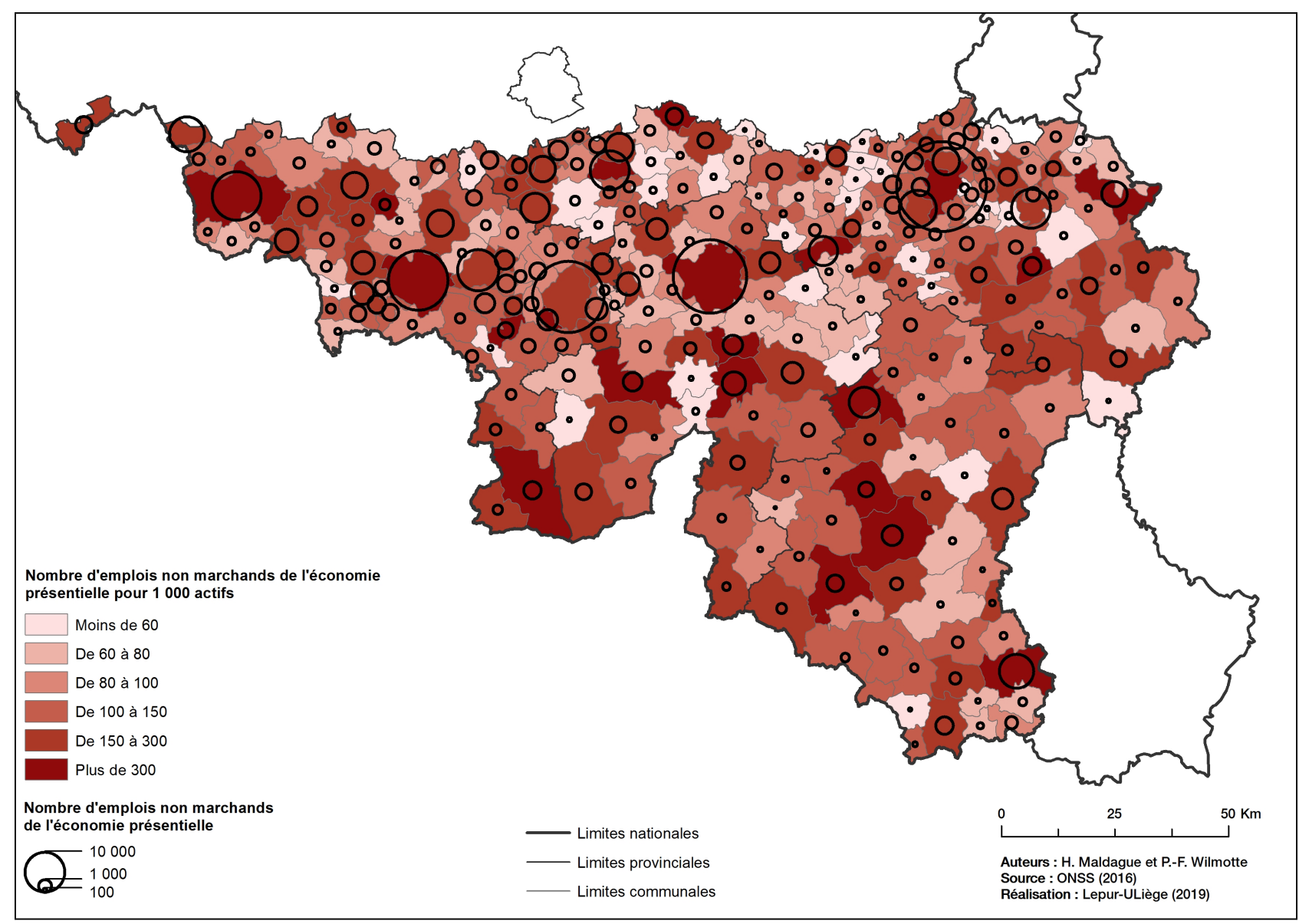

132 J.-B. JEHIN, «Influence des politiques des pouvoirs publics en matière d'implantations commerciales sur l'activité en centre-ville. Comparaison : Liège, Maastricht et Aix-la-Chapelle », Bulletin de la Société géographique de Liège, volume 42, n 1, 2002, p. 39-51 ; J.-M. LAMBOTTE, G. DEVILLET, « Le commerce», op. cit. 
Les employeurs du secteur hospitalier comptent désormais parmi les plus gros employeurs de Wallonie. Selon Leodica, ces employeurs occupent huit des dix premières places parmi les employeurs, la première place étant occupée par GSK (qui appartient également au secteur de la santé). Le secteur hospitalier est très structurant pour les villes de Liège et de Namur. Il est également très structurant lorsqu'il est fortement implanté dans des petites villes (Marche-en-Famenne, Dinant, Chimay) ou en milieu rural (CHU de Mont-Godinne au sud de Namur, centres d'accueil spécialisés comme Lierneux ou Bertrix). Les secteurs de l'administration, de l'enseignement et de la culture sont aussi concentrés dans les villes. Ils participent d'ailleurs grandement tant à la hiérarchie urbaine qu'à l'attractivité des noyaux traditionnels.

Les activités non marchandes sont structurantes pour de nombreux pôles situés le long de la frontière française, dans les provinces de Hainaut, de Namur et de Luxembourg. Dans les provinces de Liège et de Brabant wallon, ces activités sont davantage concentrées dans quelques pôles. En conséquence, ces deux provinces se caractérisent également par des espaces ruraux dans lesquels le présentiel non marchand est peu développé, comme la Hesbaye ou le Condroz entre Namur, Liège et Marche-en-Famenne.

\subsubsection{Le chômage}

Les revenus de remplacement, en particulier les allocations de chômage, représentent une des composantes de l'économie résidentielle. Dans certains territoires marqués par des taux de chômage élevés, il peut même s'agir d'une composante fondamentale. La carte 17, qui porte sur le taux de chômage administratif ${ }^{133}$ en 2014, a été dressée afin de clarifier cette thématique.

La géographie du taux de chômage correspond globalement à la géographie des bas revenus (cf. Cartes 13 et 14). Comme pour les bas revenus, des taux de chômage élevés s'observent dans deux types de territoires. Il s'agit d'abord de communes rurales qui conjuguent l'éloignement aux grands pôles d'emplois à un développement limité du tourisme. Le centre et le sud de l'Entre-Sambre-et-Meuse correspondent à ce profil, de même que l'axe de la Famenne. Le long de cet axe assez éloigné des grandes villes (Luxembourg, Namur ou Liège), le marché de l'emploi est moins favorable que dans des villes comme Ciney, plus proche de Namur, ou Libramont, plus proche de LuxembourgVille.

De hauts niveaux de chômage caractérisent également le sillon de tradition industrielle. Comme pour la géographie des bas revenus, l'extension spatiale de ce phénomène est particulièrement importante dans le Hainaut (depuis le Borinage jusque Châtelet), mais les taux de chômage sont également très élevés dans les nombreuses communes de la province de Liège historiquement marquées par les secteurs industriels traditionnels. Cette situation problématique trouve son origine dans la désindustrialisation de ces territoires et, comme nous l'avons signalé dans l'état de l'art, les hauts taux de chômage s'y perpétuent en raison du déclin de l'attractivité résidentielle et du départ des jeunes travailleurs les plus diplômés et qualifiés.

133 Le taux de chômage administratif rend compte de la proportion des personnes qui, dans la population active, sont sans emploi, à la recherche d'un emploi et disponibles pour occuper un emploi. 
Carte 17. Taux de chômage administratif (2014)

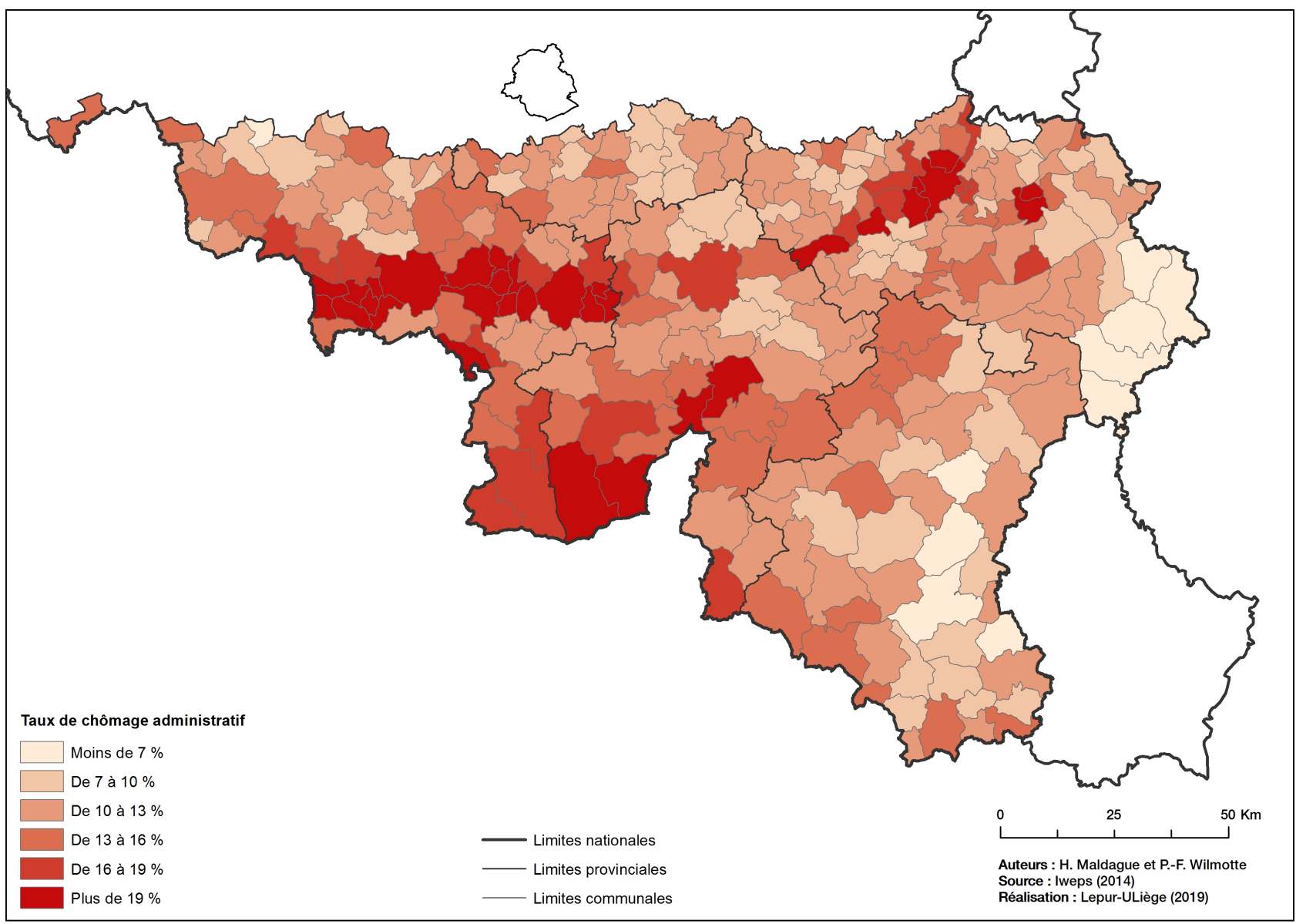

Dans le sillon de tradition industrielle, le chômage est particulièrement important dans les communes où la base productive a désormais complètement disparu, mais il est aussi très élevé dans les grandes villes de Charleroi et de Liège, qui offrent pourtant des volumes d'emploi importants. Cette situation témoigne, à l'image de la situation bruxelloise, de l'inadéquation entre les emplois offerts et les qualifications des populations résidentes.

La géographie du taux de chômage met cruellement en avant la question de l'attractivité résidentielle des villes et des communes urbaines. Force est ici de constater la dualisation entre une Wallonie des villes et une Wallonie des banlieues périurbaines. Alors que la première concentre une population davantage précarisée, la seconde bénéficie de revenus élevés et d'un chômage inférieur à la moyenne régionale. 


\subsection{TYPOLOGIE DES COMMUNES WALLONNES EN FONCTION DE LEURS PROFILS PRODUCTIFS ET/OU PRÉSENTIELS}

Pour terminer ce chapitre, nous proposons une typologie des communes wallonnes reflétant leurs profils productifs et/ou présentiels. Cette typologie est basée sur deux critères pour les aspects liés à l'économie productive et sur deux autres critères pour les aspects liés à l'économie présentielle. Pour ces quatre critères, nous nous sommes basés sur des indicateurs et avons considéré que le critère est atteint lorsque la valeur communale de l'indicateur dépasse la moyenne régionale. En fonction des indicateurs communaux, les communes peuvent remplir zéro, un ou deux critères pour chacun des deux profils. Sur la base des valeurs obtenues, les communes ont ensuite été réparties en neuf catégories.

Concernant le profil présentiel, nous avons utilisé les deux indicateurs suivants : le nombre de salariés dans les secteurs présentiels pour 1000 actifs et le revenu médian par habitant. Pour le profil productif, nous avons retenu la valeur ajoutée brute communale par habitant pour les secteurs de la base productive et le nombre d'entreprises des mêmes secteurs pour 1000 actifs.

Carte 18. Typologie des communes wallonnes en fonction de leurs profils productifs et/ou présentiels

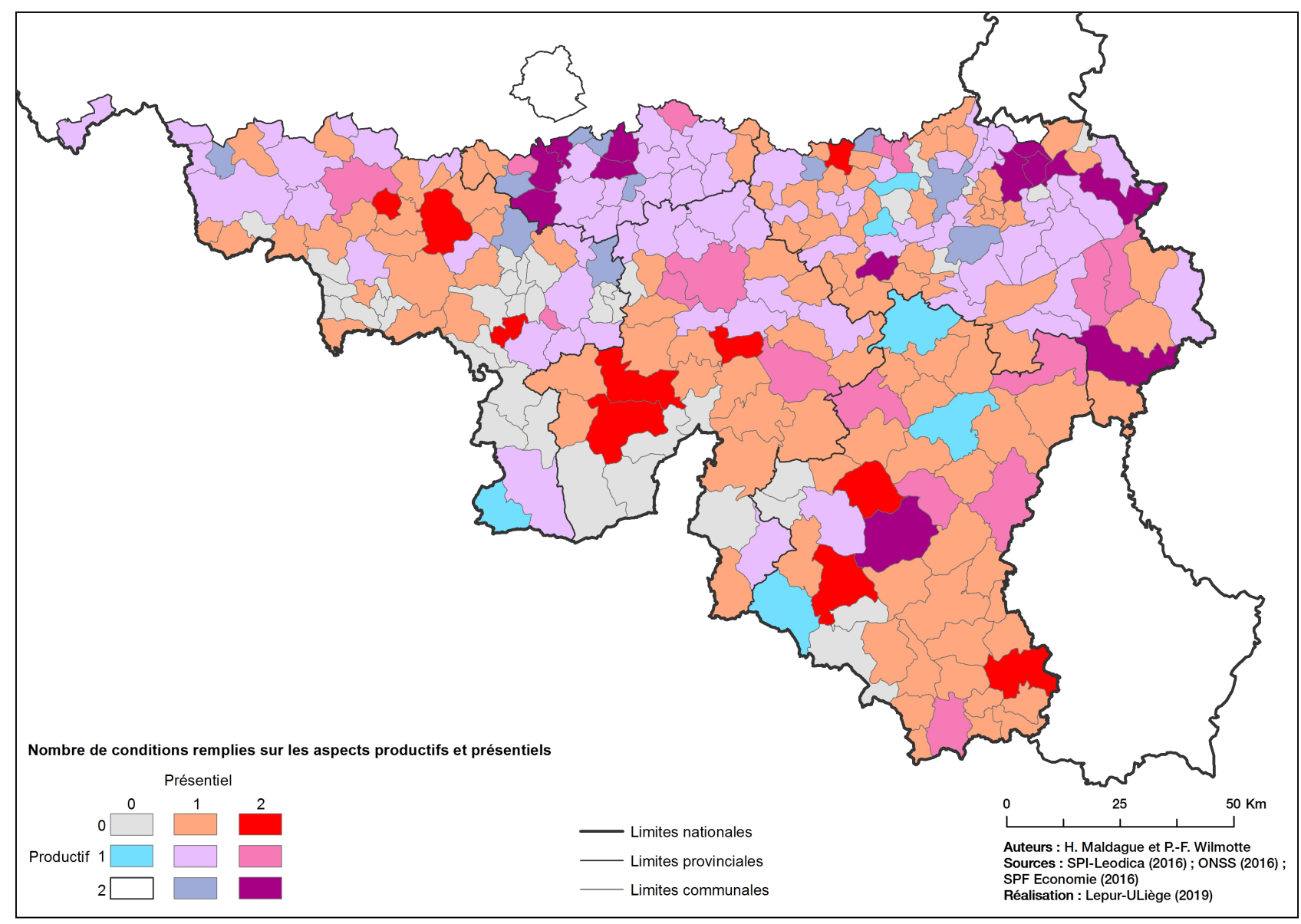


La synthèse des deux composantes est représentée sur la carte 18. L'une des neuf catégories potentielles n'y est pas rencontrée, en l'occurrence le type correspondant à des communes qui rempliraient les deux critères au niveau de l'économie productive mais aucun des deux critères au niveau de l'économie présentielle. L'inexistence de ce profil tient au fait que les communes qui présentent un profil très productif correspondent à des communes urbaines où l'on trouve des volumes relativement importants d'emplois appartenant aux secteurs présentiels, tant dans le marchand que dans le non-marchand.

Intéressons-nous maintenant aux communes qui remplissent les quatre critères. Sans surprise, une concentration importante apparaît dans le Brabant wallon, avec Wavre, Ottignies-Louvain-la-Neuve, Waterloo, Braine-l'Alleud et Nivelles. Ces communes se caractérisent par la présence de quartiers résidentiels mais aussi de pôles urbains. En outre, vu l'accessibilité autoroutière et la proximité à Bruxelles, elles sont très attractives pour les implantations d'activités. En dehors du Brabant wallon, huit autres communes remplissent les quatre critères considérés. La concentration principale apparaît dans l'Entre-Vesdre-et-Meuse, avec Herve, Aubel, Thimister-Clermont et Welkenraedt. S'y ajoutent également Libramont, Eupen et Saint-Vith (communes situées toutes deux en région de langue allemande), ainsi que Tinlot dans la banlieue périurbaine de Liège.

Quant aux communes qui sont sous la moyenne wallonne pour tous les indicateurs, deux profils émergent. Sans surprise, on trouve, d'une part, des communes rurales éloignées des plus grands pôles et situées le long de la frontière française et, d'autre part, des communes de tradition industrielle. Ce dernier type est représenté dans le Borinage, dans la région du Centre ainsi que dans les périphéries liégeoises et carolorégiennes.

La catégorie la plus représentée correspond aux entités qui remplissent un critère en économie présentielle et aucun critère en économie productive. Ce profil est présent un peu partout en Wallonie, mais particulièrement au sud de l'axe Bouillon-Bastogne, où l'influence grand-ducale induit la conjonction d'un faible secteur productif et de revenus médians élevés.

La catégorie correspondant à un critère atteint en productif et un critère atteint en présentiel est également bien représentée, notamment dans le Triangle wallon et en Wallonie picarde. On la rencontre également dans les périphéries aisées de Liège, de Charleroi et de Namur. Un classement dans cette catégorie est généralement le signe d'un certain développement.

En région liégeoise, la typologie se caractérise par de grandes disparités. On y lit la classique dualité entre les communes de tradition industrielle et les communes périurbaines plus aisées. À l'échelle de la province de Liège, on y lit également une opposition entre l'Ouest, plus résidentiel, et l'Est, plus productif.

Enfin, la carte 18 met en évidence les profils différenciés des plus grandes villes wallonnes. Cela traduit un potentiel varié quant à leur rôle de moteur productif pour elles-mêmes ou pour leur hinterland. À la lumière des indicateurs retenus, Mons semble peu générateur. Par contre, une dynamique existe à Liège, qui profite à la ville elle-même mais surtout aux petits pôles périphériques ou aux communes qui accueillent un parc d'activités. Cette dynamique reste toutefois moindre que celle du Brabant wallon et de ses pourtours. 


\section{LA STRUCTURATION DES TERRITOIRES WALLONS PAR LES AIRES MÉTROPOLITAINES TRANSFRONTALIÈRES}

Nous avons déjà souligné à différentes reprises que de vastes pans du territoire régional wallon subissent l'impact des métropoles extérieures et que, corollairement, il n'existe pas de villes wallonnes pouvant prétendre au statut de métropole complète. Dans ce cinquième chapitre, ce sont ces thématiques relatives à la métropolisation incomplète des villes wallonnes et aux dynamiques transfrontalières insufflées par les métropoles extérieures que nous allons approfondir.

Pour ce faire, nous allons débuter en dressant un état des lieux des flux de travailleurs transfrontaliers. Par travailleur transfrontalier, nous entendons les actifs qui traversent une frontière régionale ou nationale pour se déplacer entre leur lieu de résidence et leur lieu d'activité professionnelle. Parmi les transfrontaliers, nous distinguons, d'une part, les transnationaux, dont les lieux de résidence et d'activité sont situés dans des pays distincts et, d'autre part, les transrégionaux, qui résident et exercent leur profession dans un même pays, en l'occurrence la Belgique. Dans un second temps, la question des aires métropolitaines sera approfondie grâce à un focus sur chacune des aires qui ont un impact sur la Wallonie. Nous passerons alors en revue les aires de Bruxelles, de Luxembourg, de l'Euregio Meuse-Rhin et de l'Eurométropole.

\subsection{LES FLUX DE TRAVAILLEURS TRANSNATIONAUX ET TRANSRÉGIONAUX}

La Wallonie est l'une des régions européennes les plus en interaction avec ses régions voisines pour les flux de travailleurs transfrontaliers ${ }^{134}$. Pour l'année 2014, nous estimons que les flux sortants représentent $17,4 \%$ des travailleurs au lieu de résidence et que les flux entrants représentent $6,6 \%$ des travailleurs au lieu d'activité. Il ressort de ces chiffres que l'économie wallonne bénéficie d'une balance positive avec des masses importantes de flux de salaires entrants. Pour la Wallonie, cette situation contribue à expliquer les différentiels entre les deux indicateurs du PIB par habitant et du revenu par habitant. Rappelons que, pour un niveau de référence à 100 pour l'ensemble de la Belgique, le

134 Cela est encore plus important si l'on tient compte de Bruxelles d'après les calculs effectués par T. CHILLA et al., Metroborder Cross-border polycentric metropolitan regions (final report), Luxembourg, ESPON, 2010. 
premier indicateur affiche une valeur de $\pm 70 \%$ alors que le second affiche une valeur de $\pm 90 \%{ }^{135}$. Bien sûr, pour expliquer correctement la différence entre les indicateurs du PIB par habitant et du revenu par habitant, il faudrait également considérer les transferts inter-régionaux au travers du système fiscal et du régime de sécurité sociale ${ }^{136}$.

\subsubsection{Quantification des flux de travailleurs transnationaux}

Bien que le croisement de données issues de plusieurs pays rende l'analyse des flux de travailleurs transnationaux relativement complexe ${ }^{137}$, les statistiques disponibles pour la Wallonie apportent un éclairage univoque sur cette question, comme l'illustre le graphique 3 (relatif aux flux entrants et sortants).

Le graphique 3 témoigne du découplage qui s'enclenche à partir de 2008 entre les volumes d'entrants et de sortants. Auparavant, les deux courbes suivaient des trajectoires relativement parallèles, exprimant des échanges transfrontaliers toujours plus importants. Depuis 2009, le nombre de travailleurs étrangers exerçant en Wallonie s'est stabilisé alors que le nombre de travailleurs wallons exerçant à l'étranger a poursuivi sa croissance. C'est essentiellement le renforcement des flux vers le Grand-Duché de Luxembourg qui soutient cette croissance. En parallèle, la stabilisation du nombre de travailleurs français exerçant en Wallonie explique la stabilisation des flux transnationaux entrants.

Graphique 3. Évolution des flux transnationaux :

volume de travailleurs entrant en Wallonie et sortant de Wallonie (1999-2018)

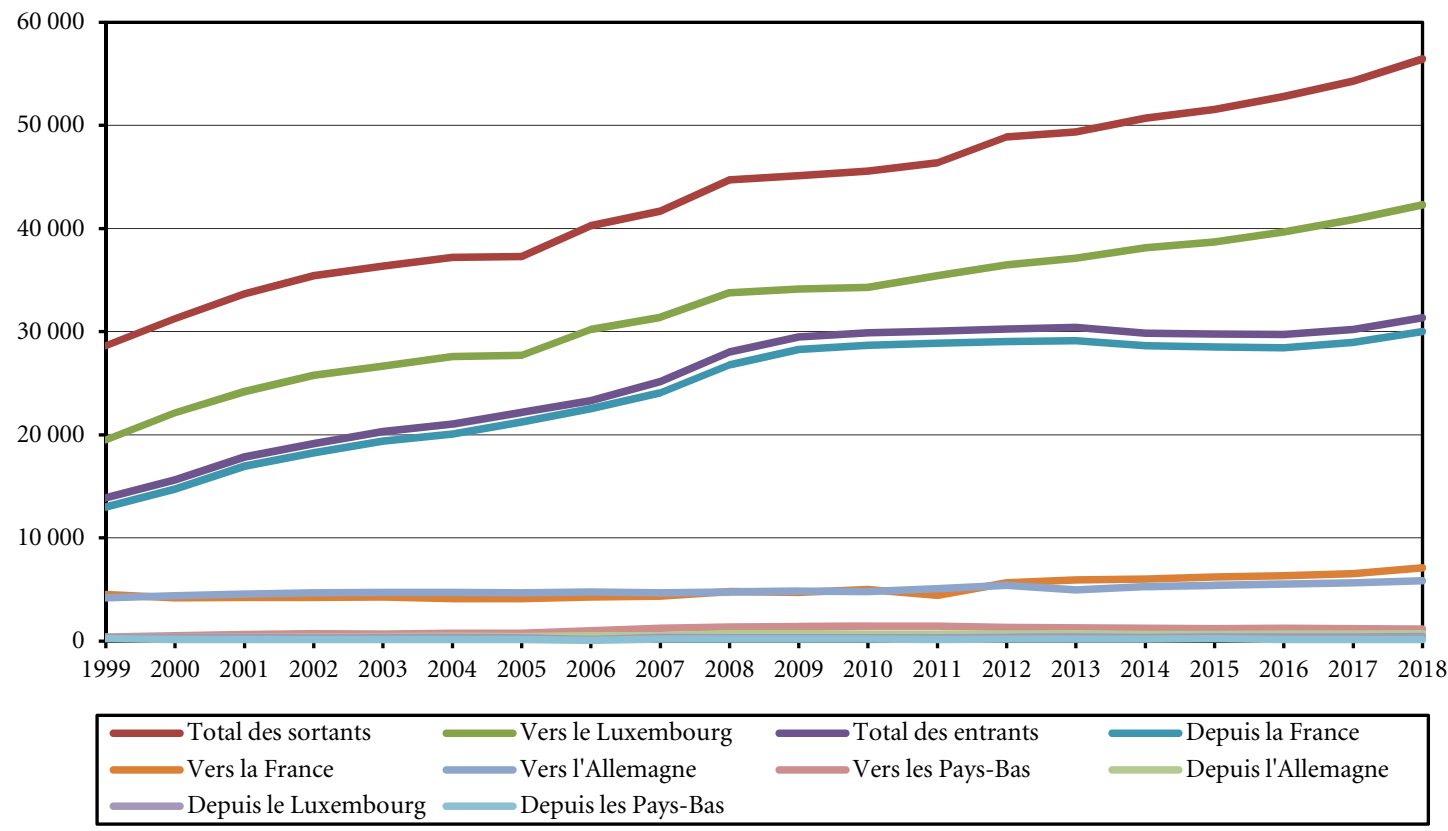

Source : INAMI.

${ }_{135}$ C. Albessart et al., Rapport sur l'économie wallonne, op. cit., p. 271.

36 O. Meunier, M. MignOlet, M.-E. Mulquin, «Une mesure des transferts interrégionaux en Belgique de 1955 à 1968 », Cahiers de recherche du CERPE. Série Politique économique, nº 11, 2007.

137 A. FEREDJ, Étude de faisabilité d'une observation pérenne des espaces transfrontaliers, Paris, UMS Riate, CIST \& CGET, 2014. 
En comparaison des flux entrants depuis la France (approximativement 30000 travailleurs) et sortants vers le Grand-Duché (approximativement 40000 travailleurs), les autres échanges sont à la fois bien moindres et plus stables. Pour les dernières années prises en compte, une hausse est toutefois notable pour les flux sortants vers la France.

En résumé, le Luxembourg est de plus en plus attractif pour les travailleurs résidant en Wallonie et le territoire wallon demeure attractif pour les travailleurs résidant en France. Le volume des flux transnationaux reste cependant en retrait par rapport aux flux transrégionaux, en particulier vis-à-vis des volumes d'échanges avec Bruxelles.

\subsubsection{Quantification des flux de travailleurs transrégionaux}

Le graphique 4 porte sur les évolutions des flux transrégionaux. À la différence du graphique 3, établi sur la base de données de la sécurité sociale, le graphique 4 est établi à partir d'une enquête, en l'occurrence l'Enquête sur les forces de travail (EFT). Il en résulte donc une variabilité interannuelle induite par la représentativité statistique limitée.

Graphique 4. Évolution des flux transrégionaux :

volume de travailleurs entrant en Wallonie et sortant de Wallonie (1999-2015)

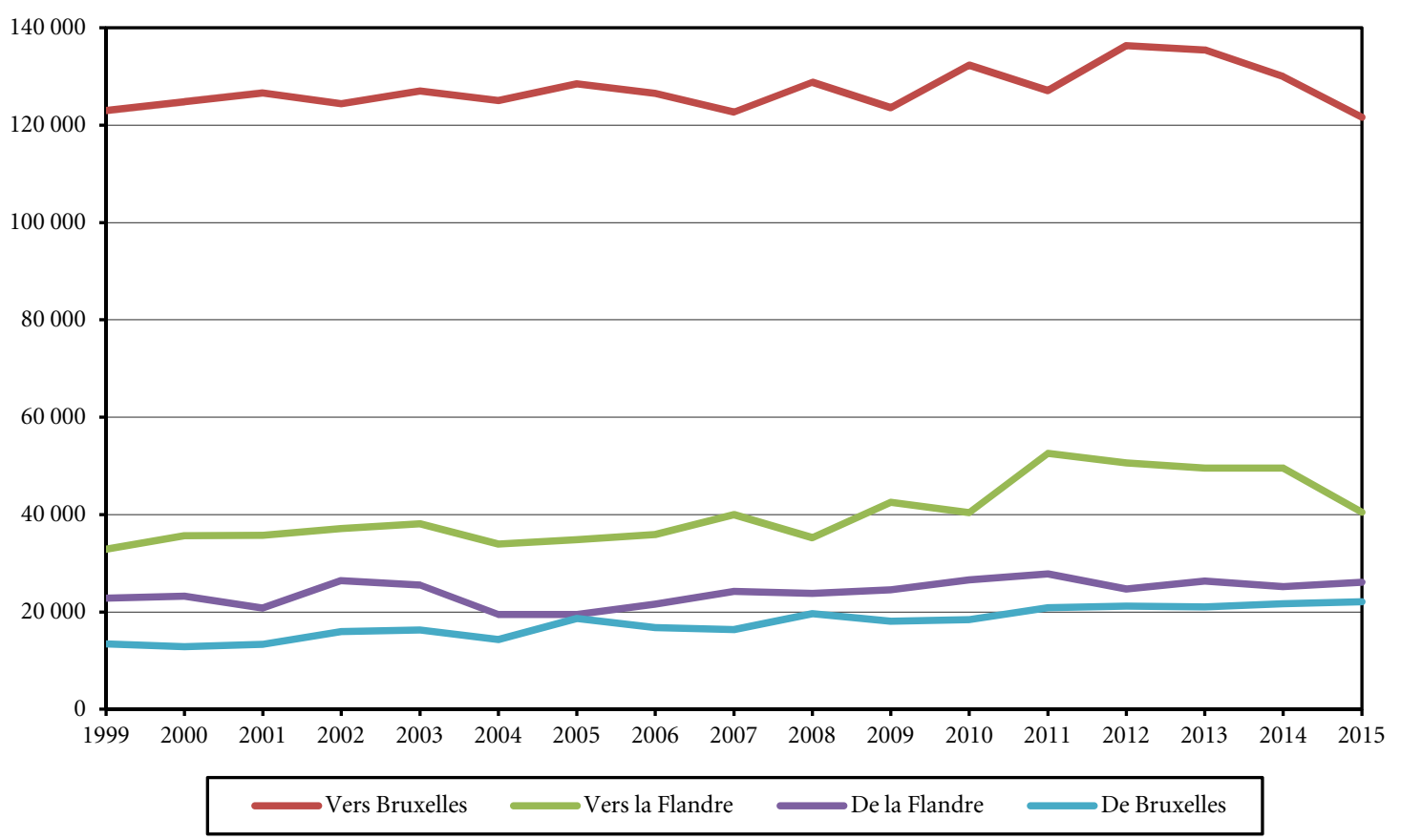

Source : Enquête sur les forces de travail (EFT).

En 2015, ce sont approximativement 120000 habitants de Wallonie qui travaillent à Bruxelles et 40000 qui travaillent en Flandre. En 2012, le nombre de travailleurs wallons actifs à Bruxelles était proche de 140000 et la décroissance est ici significative. Quoique moins nette, une même tendance à la baisse, non représentée sur le graphique 4, est également constatée pour les travailleurs qui effectuent la navette depuis la Flandre vers Bruxelles. 
Il est actuellement impossible de conclure si la tendance d'une moindre influence du pôle d'emploi de la région de Bruxelles-Capitale sur la Wallonie représente une évolution structurelle. Sur ce sujet, il convient de préciser que la dynamique productive insufflée par la métropolisation bruxelloise s'étend bien au-delà des 19 communes bruxelloises, et cela notamment dans le Brabant wallon. En conséquence, on peut considérer que la décroissance des flux vers Bruxelles traduit une influence en baisse du centre de l'espace métropolitain bruxellois (région de Bruxelles-Capitale) plutôt qu'une influence en baisse de l'ensemble de la métropole, dont le développement serait de plus en plus polycentrique ${ }^{138}$.

En 2015, les flux entrants en Wallonie représentent approximativement 25000 entrées depuis la Flandre et 22000 entrées depuis Bruxelles (cf. Graphique 4). Les flux en provenance de Bruxelles sont en hausse tendancielle sur la période analysée. En dépit des évolutions récentes correspondant à une baisse du nombre de travailleurs wallons à Bruxelles et à une hausse des travailleurs bruxellois en Wallonie, le déséquilibre des échanges entre les deux régions demeure très conséquent $( \pm 120000$ travailleurs wallons actifs à Bruxelles contre \pm 22000 travailleurs bruxellois actifs en Wallonie). Pour leur part, les flux depuis la Flandre vers la Wallonie se sont stabilisés lors des dernières années considérées, après avoir également crû entre 2004 et 2011.

L'augmentation du nombre de navetteurs bruxellois entrant en Wallonie se fait principalement à destination de la partie wallonne de l'aire métropolitaine bruxelloise, correspondant globalement à un rayon de 30 kilomètres autour de la capitale. Une dynamique semblable, mais un peu moins affirmée, est observée pour les habitants de l'aire métropolitaine bruxelloise habitant en Flandre et exerçant en Wallonie. Ces mouvements confirment l'idée que le centre et l'ouest du Brabant wallon s'intègrent de plus en plus à l'économie productive de la métropole bruxelloise. Il est donc de moins en moins pertinent de les considérer comme de simples périphéries résidentielles.

\subsubsection{La géographie de la part des flux sortants}

La carte 19 permet d'affiner l'analyse des flux sortant depuis la Wallonie par un traitement des données à l'échelle des 262 communes. Sur cette carte réalisée à partir de données datant de 2011, nous avons représenté la part des résidents wallons qui travaillent en dehors de la Wallonie.

138 Des constats de rééquilibrage économique entre les parties wallonnes, flamandes et bruxelloise de la région métropolitaine de Bruxelles ont été faits par A. LECLERCQ, A. MALHERBE, F. QUADU, « Coopération transfrontalière : la position des pôles wallons dans les aires métropolitaines transfrontalières ", Notes de recherche de la CPDT, $\mathrm{n}^{\circ}$ 62, 2015. 
Carte 19. Part des résidents wallons travaillant en dehors de la Wallonie (2011)

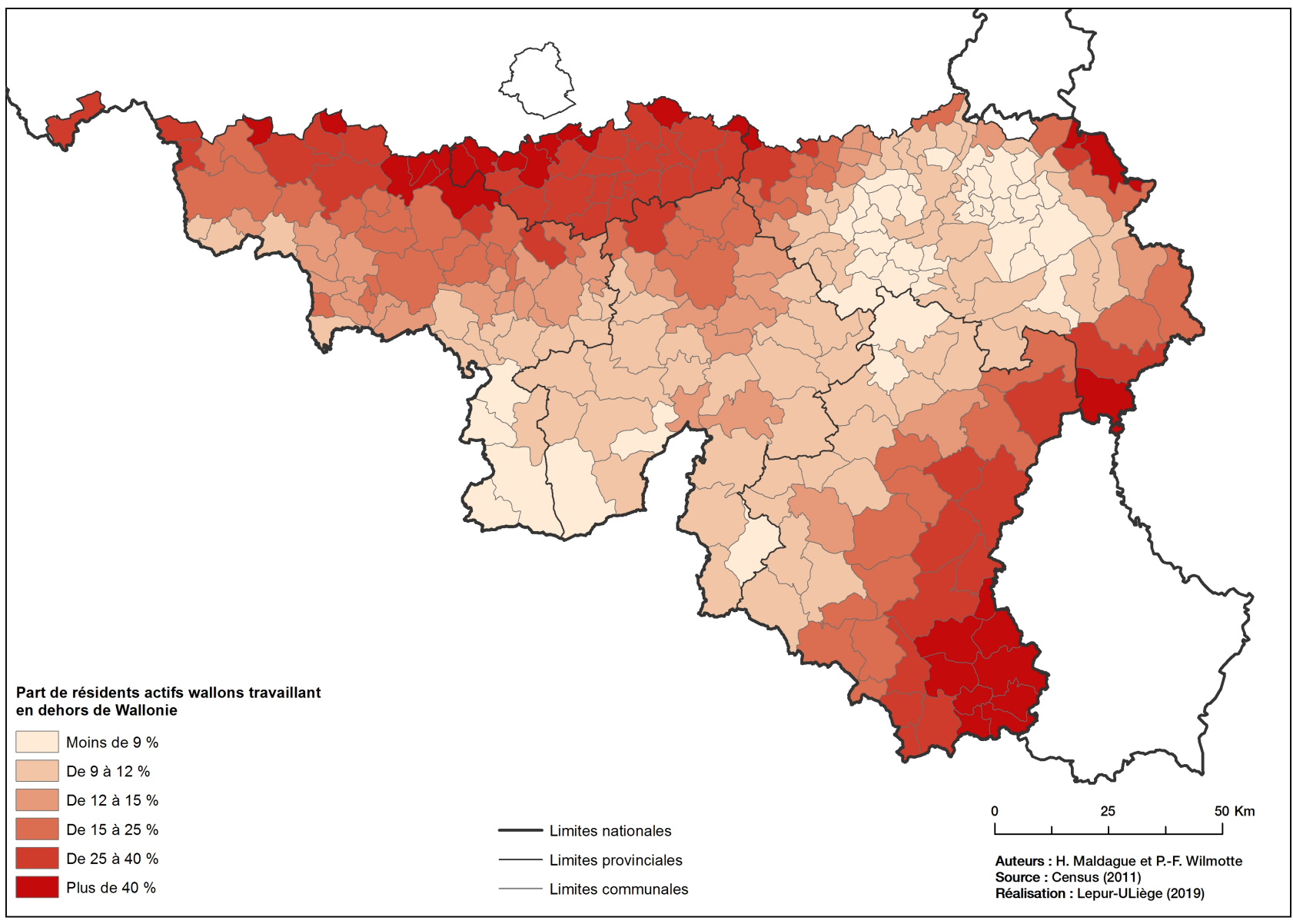

Une grande partie de la Wallonie est concernée par des flux sortants significatifs. Au nord du sillon, cela s'explique par l'attractivité de Bruxelles, mais également de la Flandre et, en particulier, du Brabant flamand. Sur la base d'une analyse spécifique sur les migrations de travail vers Bruxelles et la Flandre ${ }^{139}$, il apparaît sans surprise que la population du Brabant wallon est fortement polarisée. Cette analyse montre également que la région de Bruxelles-Capitale et son proche hinterland flamand recrutent des travailleurs depuis nombre de communes bien plus lointaines, y compris dans le Pays de Herve, dans le Condroz, ainsi que le long de l'axe Namur-Luxembourg jusque Marcheen-Famenne et Rochefort. Au final, l'influence du pôle d'emploi de Bruxelles se caractérise, d'une part, par une zone extrêmement étendue et, d'autre part, par une perte d'influence en Brabant wallon, où le développement de l'économie productive semble limiter la nécessité de rejoindre une commune bruxelloise pour accéder à un emploi ${ }^{140}$.

La carte 19 permet également de vérifier que les flux de travailleurs wallons exerçant au Grand-Duché de Luxembourg sont très importants. Ces flux sont significatifs pour une grande partie de la province de Luxembourg mais aussi pour le sud-est de la province

139 J.-M. LAMBOTTE, R. SANDU, B. BIANCHET, Recherche R1 : État du territoire wallon. Rapport scientifique partie 3/3. Structure territoriale de fait. Volet III, Namur, CPDT, 2016, p. 39.

140 A. LeClercQ, A. MALHERbE, F. QUADU, «Coopération transfrontalière », op. cit. 
de Liège. Quelques communes de la province de Namur envoient également un nombre significatif de travailleurs vers le Grand-Duché. Si la destination première est LuxembourgVille, de nombreux résidents wallons travaillent aussi dans la région d'Esch-sur-Alzette ainsi que dans les communes luxembourgeoises frontalières à la Wallonie ${ }^{141}$.

Pour les échanges avec la Flandre, les territoires wallons les plus concernés correspondent au Brabant wallon, vu l'attractivité du Brabant flamand voisin et, dans une moindre mesure, de la Wallonie picarde. Bien que son influence soit nettement plus limitée que celle de Bruxelles ou du Luxembourg, Lille exerce un pouvoir d'attraction non négligeable sur l'ouest du Hainaut (de Comines à Tournai). Aix-la-Chapelle fait de même sur le nord de la région de langue allemande, mais aussi sur Plombières, Welkenraedt et Baelen.

\section{FOCUS SUR LES AIRES MÉTROPOLITAINES}

Après avoir dressé un état des lieux sur les flux de travailleurs transfrontaliers, nous allons maintenant approfondir l'analyse par un focus sur les aires métropolitaines qui ont un impact sur la Wallonie. Rappelons qu'il s'agit des aires de Bruxelles, de Luxembourg, de l'Euregio Meuse-Rhin et de l'Eurométropole.

\subsubsection{L'aire métropolitaine de Bruxelles}

Bruxelles est le premier grand pôle attracteur extérieur à la Wallonie. Son influence tant productive que présentielle s'exerce sur un territoire très vaste. Le passage en revue des différents indicateurs socio-économiques traités pour le présent Courrier hebdomadaire conduit à mettre en évidence un territoire aux limites floues mais discernables, où l'on note une concentration d'emplois, d'entreprises, de services supérieurs, d'activités métropolitaines et de dépenses en investissement R\&D plus importante qu'ailleurs en Wallonie. Ce territoire correspond globalement à un triangle s'étendant depuis Bruxelles et le nord du Brabant wallon jusqu'au sillon wallon entre Namur et Mons.

Malgré les clivages politiques et administratifs du pays, Bruxelles reste - et de loin - la première concentration d'activités économiques, d'emplois et de service en Belgique, avec un pouvoir économique qui s'exerce sur une très grande partie du territoire belge ${ }^{142}$. Ce territoire de l'aire métropolitaine bruxelloise, aussi appelée espace Europolis ${ }^{143}$, intègre le Triangle wallon ainsi que le Diamant flamand.

Le Triangle wallon est toutefois loin d'être un espace homogène. Son intégration complète à la métropole bruxelloise est régulièrement discutée, en particulier le hiatus entre le Brabant wallon et l'ancien sillon industriel wallon. En outre, des disparités existent

141 J.-M. LAMBOtTe, R. SANDU, B. BIANCHET, Recherche R1 : État du territoire wallon. Rapport scientifique partie 3/3. Structure territoriale de fait. Volet III, op. cit.

${ }_{142}$ J.-F. Thisse, I. ThOMAS, «Bruxelles et Wallonie », op. cit., p. 75-93.

143 J. DE SALLE, Une autre échelle pour Bruxelles. L'espace Europolis Bruxelles. Création d'un nouvel espace métropolitain d'envergure internationale au cour de l'Europe du Nord-Ouest, Bruxelles, Ministère de la Région de Bruxelles-Capitale, 2009. 
également le long du sillon. À l'est et au centre, Namur profite de son statut de capitale de la Wallonie et Charleroi bénéficie de son accessibilité aisée au Brabant wallon et à Bruxelles. À l'ouest, le déficit de dynamique économique des régions de Mons et de La Louvière pose la question de la continuité du Triangle wallon à sa base, ce dernier demeurant aujourd'hui moins intégré et cohérent que le Diamant flamand ${ }^{144}$.

L'ouest et le centre du Brabant wallon occupent la place privilégiée du « sommet » du Triangle wallon. Ces zones tirent le plus grand profit de la dynamique métropolitaine bruxelloise en accueillant les plus grandes concentrations d'emplois qualifiés et d'activités métropolitaines. Toutefois, même au sein de cet espace, les activités se répartissent de manière hétérogène et plusieurs polarités se démarquent : le bipôle Wavre-OttigniesLouvain-la-Neuve, la conurbation Braine-l'Alleud-Waterloo, Nivelles et, dans une moindre mesure, l'ensemble La Hulpe-Rixensart.

Parmi ces polarités, l'ensemble formé par Wavre et Ottignies-Louvain-la-Neuve est le plus important. L'Université catholique de Louvain (UCLouvain) et les grandes entreprises qui se sont implantées et développées dans ces deux communes contribuent à faire émerger celles-ci comme le premier pôle démographique et économique du Brabant wallon. Aujourd'hui, les deux communes concentrent environ 40000 emplois (salariés et indépendants à titre principal) pour 65000 habitants. À titre de comparaison, cela représente un volume d'emplois supérieur à celui de la ville de Mons, malgré son double statut de ville universitaire et de chef-lieu de la province wallonne la plus peuplée. La croissance du bipôle Wavre-Ottignies-Louvain-la-Neuve est fortement stimulée par les présences de GSK et de l'université louvaniste, qui compte aujourd'hui plus de 30000 étudiants, dont les deux tiers sur le campus brabançon.

La dynamique du sommet du Triangle wallon est bien sûr liée à la proximité de Bruxelles. Toutefois, une part croissante de ce développement apparaît de nature endogène et le développement de l'économie productive joue un rôle majeur dans la bonne santé de ce poumon économique wallon. Ce développement productif diffuse aujourd'hui au sein des communes environnantes, voire en dehors de la province de Brabant wallon, par un mouvement de périurbanisation. Ce mouvement s'accompagne d'une croissance de l'économie résidentielle, du développement d'activités indépendantes diverses et de la création de nombreuses PME, souvent de très petite taille. Des petites villes comme Soignies, Braine-le-Comte et Gembloux doivent leur dynamisme démographique et d'emplois au double jeu du développement endogène du Brabant wallon et de l'influence de la métropole bruxelloise.

Au final, les deux composantes structurantes du Triangle wallon, la base formée par le sillon wallon et le sommet formé par le Brabant wallon, ne doivent pas être appréhendées comme des espaces homogènes. Elles sont à considérer comme des concentrations de polarités démographiques et économiques reliées entre elles par des axes de transport qui contribuent à leur développement et à l'extension progressive des rayonnements bruxellois et brabançon vers Mons, Charleroi et Namur.

144 M. VAN MEETEREN et al., « Flemish diamond or ABC-axis? », op. cit., p. 974-995. 


\subsubsection{L'aire métropolitaine de Luxembourg}

Lors des dernières décennies, la croissance du Grand-Duché de Luxembourg et de sa capitale a eu de plus en plus d'impacts sur le sud-est de la Wallonie. Pour autant, les retombées économiques en province de Luxembourg et sur ses marges liégeoises et namuroises sont bien différentes de celles insufflées par la logique métropolitaine bruxelloise sur le Brabant wallon ou, plus largement, sur le triangle éponyme. S'il est relativement aisé d'identifier le dynamisme productif du Triangle wallon et, dans une moindre mesure, de la région de Liège, ce n'est pas le cas du sud-est de la Wallonie. En effet, en termes d'économie productive, l'espace au sud de l'axe Bouillon-LibramontBastogne présente des caractéristiques similaires aux parties les moins dynamiques de la province de Hainaut.

Précisons le propos en invoquant les différences en termes de catégories d'emplois entre le Brabant wallon et le sud de la province de Luxembourg, qui possèdent pourtant tous deux un pourcentage élevé de détenteurs d'un diplôme de l'enseignement supérieur. La proportion d'emplois du secteur productif est bien plus importante dans le Brabant wallon que dans le Sud-Luxembourg. Cela s'explique par le fait qu'une grande partie de la population diplômée qui réside dans le Luxembourg belge travaille de l'autre côté de la frontière. En outre, ce phénomène tend à se renforcer car la création d'entreprises dans la partie belge de l'aire métropolitaine de Luxembourg chute ${ }^{145}$ (cf. infra, 6.2). Dans le même temps, le nombre de travailleurs frontaliers vers le Grand-Duché continue sa croissance.

Du point de vue de l'économie productive, l'opposition entre le dynamisme du Brabant wallon et l'atonie de la province de Luxembourg est saisissante. Bien sûr, les avantages fiscaux du Grand-Duché proposés aux entreprises et aux travailleurs n'y sont pas étrangers ${ }^{146}$. Ainsi, le sud-est de la Wallonie prend de plus en plus les traits de la grande périphérie résidentielle de Luxembourg, au fur et à mesure de l'absorption d'une partie de l'activité économique productive par le voisin grand-ducal et de la croissance du nombre de travailleurs frontaliers. En outre, les concurrences dans le secteur du commerce de détail entre le Grand-Duché et la Wallonie ${ }^{147}$, ainsi que le comportement du travailleurconsommateur qui ne consomme pas forcément près de son lieu de résidence, remettent en question le potentiel de développement de l'économie présentielle marchande dans cette partie du territoire wallon ${ }^{148}$.

\subsubsection{L'aire métropolitaine potentielle de l'Euregio Meuse-Rhin}

En 1976, l'Euregio Meuse-Rhin, l'une des premières eurorégions de coopération transfrontalière, voyait le jour au départ d'une volonté de coopération transfrontalière

145 J.-M. LAмвотте et al., Schéma de développement territorial de la Grande Région. Cahier thématique $n^{\circ} 3$ : Développement économique, op. cit., p. 13.

146 L. FonTAgné, Compétitivité du Luxembourg: une paille dans l'acier, Luxembourg, Ministère de l'Économie et du Commerce extérieur du Grand-Duché de Luxembourg, 2005.

147 E. EvRARD, C. SCHULZ, «L'ambition métropolitaine: clé vers un aménagement du territoire transfrontalier en Grande Région SaarLorLux ? ", L'Information géographique, volume 79, n 3, 2015, p. 54-78.

148 G. DREVON et al., "Cross-border integration and from trip chains to profiles of cross-border workers ", BIVEC-GIBET Transport Research Days, Luxembourg, 2013. 
accrue entre la province de Liège, le Limbourg belge, la partie sud de son homonyme néerlandais, la Communauté germanophone et l'association REGIO Aachen regroupant cinq Kreise (cercles administratifs) allemands. Dans cet ensemble, le " grand Liège » faisait office de potentiel premier pôle vis-à-vis d'Hasselt, de Maëstricht et d'Aix-la-Chapelle. Quarante ans plus tard, Liège apparait peu intégrée à cet ensemble et les flux de navetteurs avec les trois autres parties de l'Euregio restent négligeables. La dynamique de métropolisation, plus importante à Maëstricht et à Aix-la-Chapelle, tend à marginaliser Liège, qui a pris plus de temps à digérer le déclin industriel et qui peine à se positionner dans l'internationalisation des villes ${ }^{149}$.

Une illustration de ce risque de marginalisation est pointée par l'Organisation de coopération et de développement économiques (OCDE) au travers du projet de la TopTechnology Region Eindhoven-Leuven-Aachen Triangle (TTR-ELAt), qui associe les institutions politiques, économiques et de recherche autour de l'innovation dans l'Euregio Meuse-Rhin. Les acteurs liégeois peinent manifestement à prendre place au sein de cette structure ${ }^{150}$ orientée autour de la R\&D et impulsée par des universités particulièrement performantes aux échelles internationales et mondiales ${ }^{151}$. Pourtant, Liège dispose d'un potentiel urbain fort et d'un tissu économique dont le potentiel productif n'est pas négligeable. En outre, Liège et son bassin peuvent s'appuyer sur la relative bonne performance économique des petites villes situées entre Liège, Aix-la-Chapelle et Maëstricht, que ce soit dans le Pays de Herve, dans la périphérie verviétoise ou dans les communes germanophones.

Pour d'autres fonctions, comme les commerces et les loisirs, la frontière entre la province de Liège et ses voisins est plus poreuse. Comme nous l'avons déjà constaté, Maëstricht attire un nombre non négligeable de Liégeois pour son centre commerçant tandis que Liège reste un attracteur non négligeable sur les territoires néerlandais et allemands notamment pour certaines manifestations culturelles ou commerciales d'importance (le marché de la Batte est bien connu au-delà des frontières). Aix-la-Chapelle attire également de nombreux chalands belges dans ses commerces, notamment pour l'offre alimentaire.

Nous pouvons formuler quelques hypothèses expliquant l'intégration incomplète du " grand Liège » dans l'Euregio. La première est probablement la barrière de la langue, grand frein aux échanges économiques transfrontaliers. Peu de Liégeois maîtrisent à la fois le néerlandais et l'allemand en plus de leur langue maternelle. La seconde hypothèse réside peut-être dans le déficit d'attractivité urbaine par rapport à des villes comme Maëstricht et Aix-la-Chapelle, qui présentent un cadre et des institutions à vocation métropolitaine plus attractives pour les différents usagers. Enfin, les villes néerlandaises

149 A. MALHERBE, Mutations et ressources de territorialisation de l'espace transfrontalier Meuse-Rhin sur le temps long : vers une métropole polycentrique transfrontalière?, Thèse de doctorat, UCL, 2015.

150 C. Nauwelaers, K. Maguire, G. Marsan, «The Case of the Top Technology Region/Eindhoven-LeuvenAachen Triangle (TTR-ELAt). Regions and Innovation: Collaborating across Borders », OCDE Regional Development Working Papers, $\mathrm{n}^{\circ}$ 22, 2013 (cf. notamment l'observation des auteurs selon laquelle «l'engagement politique de la Province de Liège envers le TTR-ELAt doit être clarifié » : ibidem, p. 29).

151 Par exemple, la Rheinisch-Westfälische Technische Hochschule Aachen (RWTH Aachen : école supérieure polytechnique de Rhénanie-Westphalie d'Aix-la-Chapelle) est dans le top 3 allemand des universités techniques dispensant un cursus d'ingénieur. Nous estimons que près de $10 \%$ des ingénieurs actifs en R\&D en Allemagne se concentrent dans la région d'Aix-la-Chapelle, qui compte pourtant moins de $1,5 \%$ de la population du pays. 
et allemandes ont opéré des choix porteurs quant à leur internationalisation, leur permettant de générer une dynamique métropolitaine plus importante qu'en région liégeoise.

\subsubsection{L'aire métropolitaine potentielle de l'Eurométropole Lille-Courtrai-Tournai}

De manière analogue à l'Euregio Meuse-Rhin, l'Eurométropole vise une coopération transfrontalière accrue entre l'agglomération de Lille et ses territoires limitrophes, à savoir la Wallonie picarde du côté wallon et la région de Courtrai du côté flamand. Sur le plan socio-économique, le pan wallon de l'Eurométropole apparaît moins bien loti que ses pendants français et flamands. En comparaison de l'aire métropolitaine de Luxembourg, les déséquilibres sont toutefois moindres ${ }^{152}$. L'aire urbaine de Lille, qui englobe entre autres Roubaix et Tourcoing, rassemble plus d'un million d'habitants. Elle représente le poids lourd démographique de l'ensemble et elle concentre un grand dynamisme économique en profitant de sa position de carrefour entre la France, la Grande-Bretagne et la Belgique. Le pan wallon apparaît ainsi en situation périphérique du centre de gravité de l'ensemble, à l'exception peut-être de Mouscron.

Par rapport aux arrondissements d'Ath et de Tournai, l'arrondissement de Mouscron est, des trois arrondissements de la Wallonie picarde, celui qui profite le plus du dynamisme économique de l'Eurométropole. Sa localisation géographique est ici idéale, dans le continuum bâti de Courtrai à Lille et sur l'axe reliant la métropole lilloise à Gand et à Anvers, autres territoires en bonne santé économique. Cette situation explique l'attractivité de Mouscron pour les entreprises flamandes, qui sont aussi très intéressées par la disponibilité tant en main-d'œuvre qu'en foncier à vocation économique ${ }^{153}$. Ces dernières années, la disponibilité en offre foncière tend toutefois à s'y réduire ${ }^{154}$. L'arrondissement de Tournai, malgré son appartenance à l'Eurométropole, semble par contre quelque peu à l'écart des dynamiques économiques les plus importantes, sans pour autant nier le potentiel économique qu'a révélé l'analyse sur la base productive (cf. supra, 4.1.2).

Malgré un potentiel a priori plus faible qu'à Courtrai (écartement de l'axe historique d'urbanisation, dynamique urbaine plus faible, peu d'institutions de recherche), la Wallonie picarde est attractive et recrute des travailleurs français et, dans une moindre mesure, des travailleurs flamands. En réalité, c'est en Wallonie picarde que réside une grande partie de l'attractivité wallonne pour les travailleurs français. Son déploiement futur passera aussi probablement par sa position sur l'axe entre Bruxelles et Lille, vu l'influence conjointe des deux pôles dans l'attraction des travailleurs, en complémentarité avec son statut de territoire de l'Eurométropole.

152 F. DURAND, "Theoretical Framework of the Cross-border Space Production. The Case of the Eurometropolis Lille-Kortrijk-Tournai », Journal of Borderlands Studies, volume 30, n 3, 2015, p. 309-328 ; E. EvRARD, C. SCHUlZ, « L'ambition métropolitaine », op. cit.

153 A. VINCENT, «L'Objectif 1 en Hainaut », Courrier hebdomadaire, CRISP, n 1534-1535, 1996, p. 37.

154 J.-M. Lambotte, H. Maldague, P. Copee, R. Sandu, B. Bianchet, J.-M. Halleux, Recherche R1 : État du territoire wallon. Expertise complémentaire : évaluation des besoins en ZAE, Namur, CPDT, 2017. 


\section{LES RESSOURCES TERRITORIALES POUR LE DÉVELOPPEMENT FUTUR DE LA WALLONIE}

Grâce aux chapitres précédents, nous connaissons la situation de la Wallonie tant en termes de politiques publiques relevant du développement économique et territorial qu'en termes de logiques métropolitaines et de disparités socio-spatiales productives et présentielles. Afin de poursuivre l'analyse, nous allons maintenant nous intéresser aux forces et faiblesses de la Wallonie en termes de ressources territoriales. Pour rappel, nous avons défini la notion de ressource territoriale comme les caractéristiques des territoires qui permettent d'y attirer des flux de revenus. Dans cette perspective, nous allons passer en revue trois thématiques afin de jauger le potentiel de développement futur de la Wallonie. Ces trois thématiques correspondent au capital humain, aux dynamiques entrepreneuriales et au niveau de spécialisation dans les secteurs potentiellement les plus porteurs.

Dans le contexte de l'économie de la connaissance, la formation, la créativité et le niveau de qualification de la population sont des ressources vitales pour le développement des entreprises et pour le développement des territoires qui les hébergent. La thématique du capital humain a été précédemment discutée dans l'état de l'art. Pour débuter le présent chapitre, nous allons approfondir cette thématique avec des analyses portant sur l'ensemble de la Belgique. Ces analyses sont consacrées aux deux indicateurs du taux de diplômés de l'enseignement supérieur et de la part de la population appartenant à la « classe créative ».

Il est bien connu que les territoires qui concentrent des populations entrepreneuriales sont favorisés en termes de développement économique ${ }^{155}$. En conséquence, après nous être intéressés aux sujets des niveaux de formation et de la classe créative, nous verrons ce qu'il en est de la ressource territoriale des dynamiques entrepreneuriales. Pour ce faire, nous consacrerons la deuxième section du chapitre à l'intensité entrepreneuriale.

Certains secteurs économiques sont caractérisés par un rôle moteur dans le développement régional. C'est là le sujet que nous analyserons dans la troisième section. Nous nous pencherons d'abord sur les services aux entreprises. Vu la place qu'ils occupent dans les échanges entre les entreprises et vu leur impact sur la productivité et la compétitivité des firmes clientes, ils sont devenus vitaux pour le développement. En raison de la position transversale occupée par les entreprises du numérique dans le développement de la vie économique, les activités de ce secteur sont aussi caractérisées par un rôle moteur sur le développement et la croissance. À la suite de l'analyse dédiée aux services aux entreprises, c'est le sujet des startups que nous traiterons afin d'appréhender la thématique du numérique.

155 S. MÜLLER, «A progress review of entrepreneurship and regional development », op. cit. 


\subsection{LE CAPITAL HUMAIN}

Comme mentionné ci-dessus, la prise en compte de la ressource du capital humain va s'appuyer sur l'analyse des indicateurs du taux de diplômés de l'enseignement supérieur et de la part de la population appartenant à la «classe créative».

\subsubsection{Le taux de diplômés de l'enseignement supérieur}

Le taux de diplômés de l'enseignement supérieur est considéré par l'UE comme un critère fondamental du développement territorial. La Commission européenne en a ainsi fait une composante clé de sa stratégie de compétitivité Europe 2020 (cf. supra, 2.1.1). Pour rappel, l'objectif mis en avant au niveau européen est que, à l'horizon 2020, on trouve 40 \% de diplômés de l'enseignement supérieur dans la cohorte des 30-35 ans. Pour certains pays, l'ambition est même plus grande, comme en Belgique où l'objectif a été porté à 47 \%

Carte 20. Part des diplômés de l'enseignement supérieur (2011)

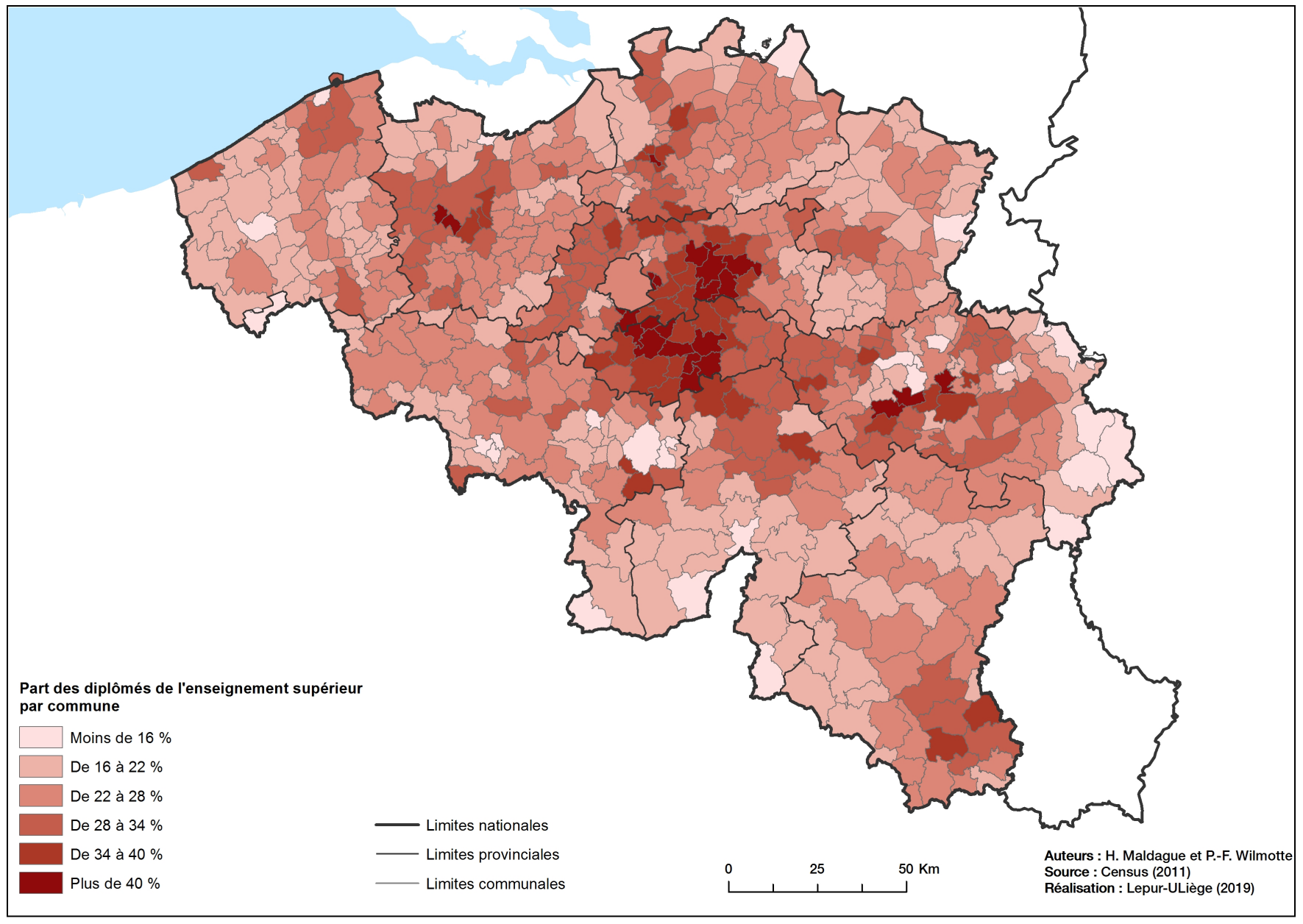


Avec la carte 20, nous approfondissons l'analyse réalisée dans le troisième chapitre sur cet indicateur de la part de diplômés de l'enseignement supérieur dans la cohorte des 30-35 ans (cf. Carte 8). Avec cette carte réalisée à l'échelle de l'ensemble de la Belgique, la variable est toutefois quelque peu différente puisque nous nous intéressons à la part des diplômés de l'enseignement supérieur dans l'ensemble de la population, en l'occurrence ici pour l'année 2011. Ce changement de variable, ainsi que le recours à l'année 2011, ont été rendus nécessaires afin de procéder à une analyse au niveau communal. Vu l'effet générationnel de l'allongement des études, les proportions de diplômés de l'enseignement supérieur dans l'ensemble de la population sont logiquement moins élevées que les proportions établies pour la seule classe d'âge des 30-35 ans.

Les écarts entre la Wallonie et la Flandre sont relativement limités pour cet indicateur des parts de diplômés de l'enseignement supérieur, avec une moyenne globale de 23,9\% en Wallonie et de 26,0 \% en Flandre (25,1\% pour la région de Bruxelles-Capitale). L'écart relativement limité indique que les différentiels de développement entre la Wallonie et la Flandre ne résultent pas d'une pénurie massive de main-d'œuvre diplômée en Wallonie. Cet écart limité indique également que le potentiel de diplômés de l'enseignement supérieur qui réside en Wallonie n'est pas suffisamment valorisé en termes de développement économique. Trois raisons nous semblent susceptibles d'expliquer cette situation. La première tient à la structuration des territoires wallons par des aires métropolitaines transfrontalières, avec en conséquence de nombreux diplômés de l'enseignement supérieur qui sont actifs en dehors du territoire régional (cf. supra). Une deuxième explication potentielle est que le profil général des diplômés de l'enseignement supérieur est insuffisamment porteur en termes de développement économique. Cette hypothèse est à mettre en lien avec certaines faiblesses «structurelles » fréquemment invoquées à propos du capital humain en Wallonie: la culture insuffisamment entrepreneuriale, la connaissance lacunaire des langues, une " ouverture au monde » qui demeure insuffisante et la pénurie en diplômés des filières techniques, scientifiques et de gestion d'entreprise ${ }^{156}$. La troisième cause possible est l'organisation générale de la vie sociale et économique. L'hypothèse est ici que des blocages de divers types empêcheraient les diplômés wallons de pleinement réaliser leur potentiel.

Sur le plan spatial, les niveaux de diplomation de l'enseignement supérieur sont particulièrement élevés dans les provinces de Brabant wallon et de Brabant flamand. Cela s'explique par le rôle des universités bruxelloises et louvanistes, mais également par la proximité aux emplois qualifiés qu'offre la zone métropolitaine bruxelloise. Concernant la Wallonie, la proximité à des emplois de niveau métropolitain permet également d'expliquer la concentration de diplômés de l'enseignement supérieur à proximité de Luxembourg-Ville. Que ce soit en Wallonie ou en Flandre, les grandes régions urbaines, et principalement leurs zones périurbaines, sont également caractérisées par de nombreuses communes où le taux de diplômés de l'enseignement supérieur est élevé. En Wallonie, il s'agit principalement des régions de Liège et de Namur, en lien avec l'offre d'enseignement présente en leur sein ou à leur proximité immédiate (pour la province de Namur, nous pensons en particulier au pôle voisin de Louvain-la-Neuve).

156 M. BourgEOIs et al., «Amélioration de l'attractivité et de la compétitivité du territoire wallon pour le secteur des services supérieurs ", op. cit. 
Pour la Wallonie, il existe une relation générale entre la variable analysée et les niveaux de revenus (cette relation est plus prégnante pour les revenus moyens que pour les revenus médians). On trouve ainsi plus de $30 \%$ de diplômés de l'enseignement supérieur dans la plupart des communes résidentielles aisées des régions urbaines de Bruxelles, de Luxembourg, de Liège, de Namur et, dans une moindre mesure, de Charleroi. La situation des villes universitaires s'écarte toutefois de cette relation générale entre niveaux de revenus et niveaux de formation. Les diplômés de l'enseignement supérieur y représentent une proportion relativement importante de la population (plus de $25 \%$ ), alors que la variable du revenu moyen y affiche des valeurs moyennes (à Ottignies-Louvain-la-Neuve) à faibles (à Namur), voire à très faibles (à Mons et à Liège). Ce décalage indique que les villes universitaires accueillent des populations importantes de jeunes adultes hautement diplômés mais que, comme l'ont montré les recherches dédiées à l'impact des cycles de vie sur la périurbanisation ${ }^{157}$, ces populations finissent souvent par les quitter lorsque leurs revenus augmentent.

On retrouve la corrélation entre revenus et diplômés de l'enseignement supérieur dans de nombreuses communes wallonnes où les deux indicateurs affichent de faibles niveaux. Cela s'observe dans les communes les plus rurales, par exemple dans le sud de la zone de langue allemande ou dans certaines entités des vallées du Viroin et de la Semois. Il s'agit ensuite de nombreuses communes où le peuplement important est héritier de la révolution industrielle. Cela concerne le Borinage, le Centre, le bassin de Charleroi et l'ouest de la région liégeoise. Pour ces communes urbaines et de tradition industrielle, le faible taux de diplomation de l'enseignement supérieur s'explique tant par un accès limité des populations locales aux études qu'au déficit d'attractivité de ces espaces de tradition industrielle sur les jeunes diplômés (en ce compris sur ceux qui en sont issus). Dans les régions du Centre et de Charleroi, ce cercle vicieux se développe à très vaste échelle. Cela justifie pleinement le signal d'alarme lancé par T. Dermine et dont nous avons rendu compte dans notre premier chapitre.

\subsubsection{La part de la population active appartenant à la classe créative}

La théorie de la classe créative a été décrite dans l'état de l'art. Nous savons qu'elle a suscité de nombreux travaux, parmi lesquels certains ont été dédiés à la Wallonie. Lors d'une recherche menée pour la Conférence permanente du développement territorial (CPDT) de la Région wallonne, Zineb Aouni, Bernard Surlemont et Fabrice Pirnay ont ainsi cherché à quantifier la part de la classe créative au départ de l'Enquête sur les forces de travail (EFT) ${ }^{158}$. Pour ce faire, ils ont appréhendé la notion de classe créative en référence

157 J.-M. HALLEUX, « Mobilité résidentielle, mobilité voiture et logiques familiales en Belgique : entre étalement de la périurbanisation et intra-urbanisation ", NETCOM, volume 19, n 3-4, 2005, p. 157-177.

158 Z. Aouni, B. Surlemont, F. PIRNAY, Recherche C3 CPDT 2012-2013: Analyse des nouvelles formes de développement de l'activité économique et de leurs liens au territoire. Annexe RC3/1: Concentration géographique de la classe créative en Wallonie: facteurs favorables et effets désirables, Namur, CPDT, 2013. L'étude repose sur l'adaptation d'une typologie de la classe créative proposée par M. LORENZEN, K. V. ANDERSEN, "Centrality and creativity: Does Richard Florida's creative class offer new insights into urban hierarchy? », Economic Geography, volume 85, n 4, 2009, p. 363-390. Nous ne développons ici que les principaux résultats de cette étude et nous invitons le lecteur intéressé par ses aspects méthodologiques à consulter l'étude en question. 
aux professions dites créatives, qu'elles soient exercées dans des secteurs créatifs ou pas. En cohérence avec les travaux de recherche réalisés en Europe sur cette thématique, les professions créatives sont définies selon la nomenclature International Standard Classification of Occupations (ISCO, dite également Classification internationale du type de professions - CITP).

Nous reprenons au graphique 5 la part de la population active appartenant à la classe créative selon la méthodologie appliquée par Z. Aouni et ses collègues. Ce graphique nous renseigne sur les parts provinciales (et pour la région de Bruxelles-Capitale) tant au lieu de résidence qu'au lieu d'activité (le classement des entités a été établi en fonction de la situation au lieu de résidence).

Graphique 5. Part de la population active appartenant à la classe créative

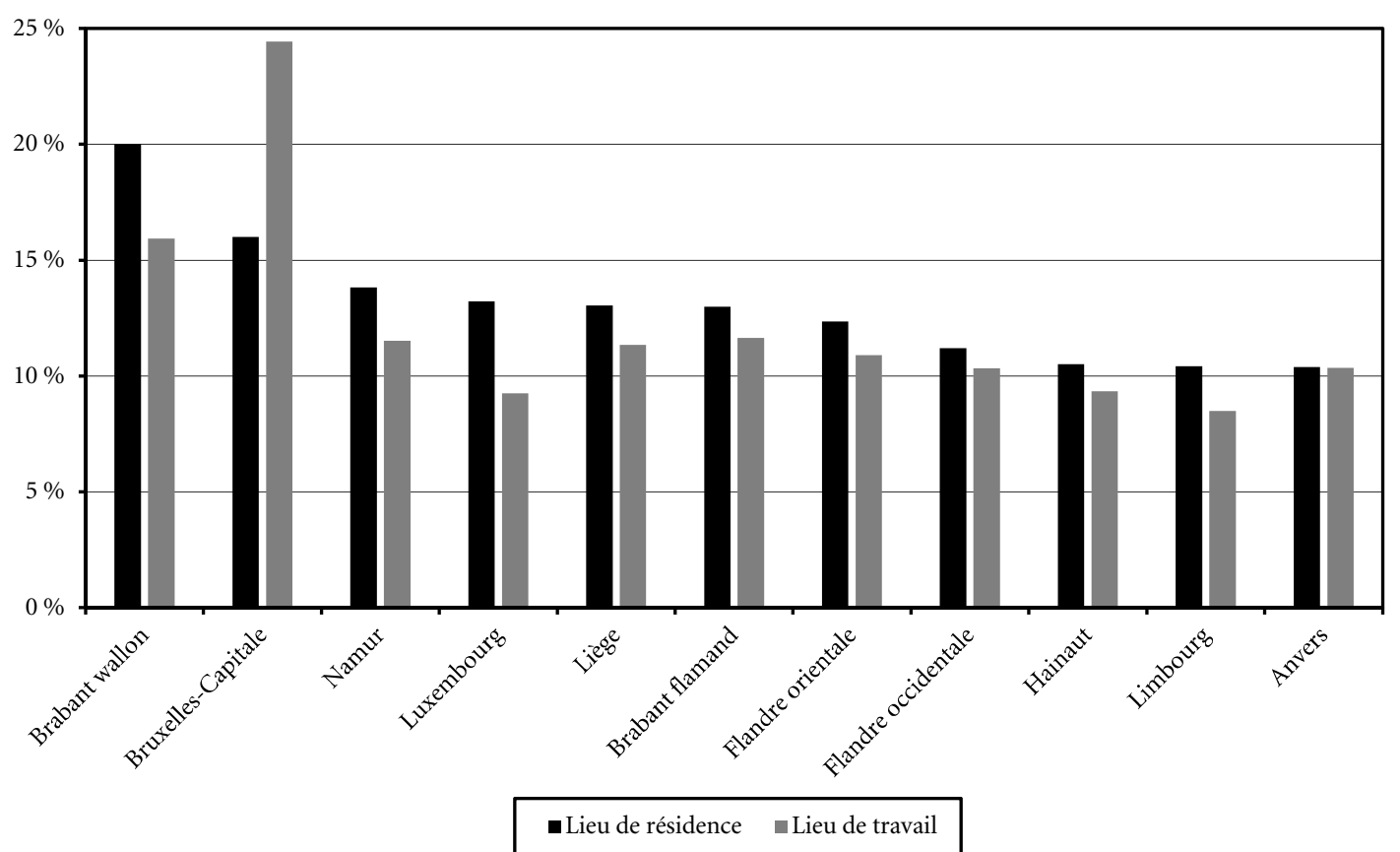

Source : Z. AOUNI, B. SURLEMONT, F. PIRNAY, Recherche C3 CPDT 2012-2013 : Analyse des nouvelles formes de développement de l'activité économique et de leurs liens au territoire. Annexe RC3/1 : Concentration géographique de la classe créative en Wallonie: facteurs favorables et effets désirables, Namur, CPDT, 2013 (sur la base des données EFT relatives à la période comprise entre 2006 et 2011).

Au lieu de résidence, les provinces wallonnes sont généralement mieux dotées en classe créative que les provinces flamandes. Bien que ce résultat soit délicat à interpréter, vu le flou qui entoure la notion de classe créative et vu la difficulté à la cerner par des indicateurs statistiques robustes, il tend toutefois à montrer qu'une proportion élevée de résidents «créatifs » n'est pas un facteur suffisant pour le développement économique des territoires. En dépit des questionnements méthodologiques, il est frappant que cette interprétation confirme les analyses menées sur la question de la classe créative à l'échelon européen ${ }^{159}$. En effet, ces analyses ont conduit à une typologie des régions NUTS 2 pour laquelle, à l'exception du Brabant wallon, les provinces wallonnes ont été regroupées dans la catégorie «main-d'œuvre hautement créative - faible PIB par habitant ». Dans

159 ESPON, « The creative workforce. Territorial dynamics in Europe », Territorial Observation, $n^{\circ}$ 5, 2011. 
cette catégorie, les provinces de Liège, de Luxembourg, de Namur et de Hainaut côtoient les pays baltes ainsi que la plupart des régions du Mezzogiorno. Pour sa part, le Brabant wallon a été regroupé avec l'ensemble des provinces flamandes au sein de la catégorie «main-d'œuvre hautement créative - haut PIB par habitant ». Ces résultats concordent avec les analyses présentées ci-dessus sur la diplomation dans l'enseignement supérieur. À l'instar de ces précédents résultats, ils interrogent sur les mesures de gouvernance à adopter afin que le potentiel de créativité du capital humain wallon puisse davantage se transformer en valeurs ajoutées et en revenus.

Le graphique 5 met également en avant la polarisation exercée par la région de BruxellesCapitale sur les navetteurs créatifs. En effet, Bruxelles est la seule entité pour laquelle la proportion de créatifs au lieu de travail dépasse la proportion de créatifs au lieu de résidence. Vis-à-vis des appartenances régionales, des proportions élevées de créatifs au lieu de travail sont aussi atteintes en Brabant wallon et en Brabant flamand. Ces résultats confirment que les travailleurs créatifs sont attirés par les opportunités en emplois supérieurs offertes par les grandes régions métropolitaines, tant par leurs centres que par leurs périphéries.

Toujours au lieu de travail, les provinces disposant d'une ville polarisante pour l'offre en enseignement supérieur affichent des taux intermédiaires (Flandre orientale, provinces d'Anvers, de Namur, de Liège). Par contre, les provinces avec des pôles urbains moins polarisants sont moins bien dotées en classe créative au lieu de travail (Hainaut, provinces de Limbourg et de Luxembourg).

Parmi les provinces wallonnes, c'est celle de Hainaut qui est la moins bien dotée en classe créative au lieu de résidence. Nous y voyons deux explications principales. D'une part, cette province reste pénalisée par une offre limitée en enseignement supérieur. D'autre part, en comparaison des provinces de Brabant wallon, de Namur et de Luxembourg, elle bénéficie d'une moindre attractivité résidentielle pour les travailleurs actifs dans les emplois liés aux deux principales métropoles qui ont un impact significatif sur la Wallonie, en l'occurrence Bruxelles et Luxembourg-Ville. En outre, il est vraisemblable que cette situation peu favorable soit liée à une faible attractivité économique et urbaine des principales villes hennuyères, qui sont en concurrence tant entre elles qu'avec Bruxelles, Lille ou Namur.

Au lieu de travail, la province wallonne la moins bien dotée n'est pas le Hainaut, mais la province de Luxembourg. Ce territoire parvient à attirer des travailleurs créatifs, mais il ne parvient pas à attirer les emplois créatifs. Cela s'explique tant par son caractère rural que par la polarisation exercée par le Grand-Duché de Luxembourg. Le profil du Brabant wallon est plus équilibré, avec des taux élevés de classe créative tant au lieu de résidence qu'au lieu de travail. Un potentiel de développement productif local y demeure toutefois inexploité puisque la proportion de créatifs au lieu de résidence demeure supérieure à la proportion de créatifs au lieu de travail.

La province de Liège correspond à un classement médian parmi les provinces belges, tant au lieu de résidence qu'au lieu de travail. Nous interprétons ce classement médian par la concomitance de facteurs d'attractivité et de répulsivité. Parmi les facteurs d'attractivité, nous trouvons la présence d'infrastructures et d'aménités urbaine de dimension métropolitaine (université, culture, etc.). Par contre, la région liégeoise reste répulsive car son marché de l'emploi continue à subir l'impact des problèmes structurels rencontrés 
dans les RETI (reconversion incomplète de la base économique, important chômage de longue durée, etc.). Dans un contexte différent, la province de Namur se caractérise par des parts semblables à celles de la province de Liège. On n'y trouve pas les mêmes équipements métropolitains, mais son marché du travail a moins subi l'impact des crises des industries traditionnelles. En outre, une partie importante du territoire provincial profite de la proximité avec le Brabant wallon et Bruxelles.

\subsection{LES DYNAMIQUES ENTREPRENEURIALES ET L'INDICE D'INTENSITÉ DE L'ENTREPRENEURIAT}

La carte 21 permet de préciser la géographie des dynamiques entrepreneuriales. L'indice retenu pour cette carte correspond au rapport entre le nombre d'entreprises et le nombre d'actifs. Cet indice d'intensité de l'entrepreneuriat se base sur le nombre d'entreprises reprises dans la base de données Leodica ${ }^{160}$. En complément de l'indice, la carte 21 rend également compte du nombre absolu d'entreprises par entité communale. Sur le plan méthodologique, il est utile de préciser que la source utilisée ne permet pas de tenir compte $\mathrm{du}$ fait que certains sièges sociaux soient localisés au lieu d'habitation du chef d'entreprise et non au lieu d'activité.

Pour l'ensemble de la Wallonie, l'indicateur considéré affiche une moyenne de 27 entreprises pour 1000 actifs. De très grandes disparités communales se cachent derrière cette moyenne, avec un rapport de 1 à 10 entre les communes les moins et les plus entrepreneuriales. Les communes les moins bien classées correspondent à trois catégories : primo, les communes anciennement industrielles situées dans les banlieues industrielles des grands pôles traditionnels; secundo, les communes proches d'Aix-la-Chapelle et de Luxembourg-Ville; tertio, des communes rurales éloignées des pôles urbains majeurs (notamment à proximité de la botte de Givet).

L'impact sur l'entrepreneuriat de la proximité au Grand-Duché de Luxembourg et à la ville d'Aix-la-Chapelle pose la question du rôle des frontières sur le développement économique de larges parties de la Wallonie. Cette question est particulièrement interpellante pour le Sud-Luxembourg, où l'on trouve l'extension spatiale la plus importante de communes peu entrepreneuriales. Dans cette partie du territoire, les ratios entre le nombre d'entreprises et le nombre d'actifs sont aussi faibles que dans le Borinage, alors que le profil de sa population se rapproche de celui du Brabant wallon. Pour le Sud-Luxembourg, la prise en compte de données sur les entreprises créées depuis 2011 montre que la crise de l'entrepreneuriat s'y accentue. En outre, ce diagnostic tranche vis-à-vis de la situation du début des années 2000 , période durant laquelle une réelle dynamique entrepreneuriale se développait dans cette partie du territoire régional ${ }^{161}$. Cela indique que l'évolution vers une marginalisation productive de ces territoires sous l'influence grand-ducale n'est pas inexorable. Il serait donc intéressant d'approfondir l'analyse afin de dégager d'éventuelles pistes de solution.

160 Rappelons qu'il s'agit des entreprises qui disposent de plus de 1000 euros d'actifs inventoriés.

161 V. Calay, J.-L. GuYot, G. Van Hamme, Capital humain et inégalités spatiales de la création d'entreprise : le cas de la Wallonie, Namur, IWEPS. 
Carte 21. Indice d'intensité de l'entrepreneuriat (2016)

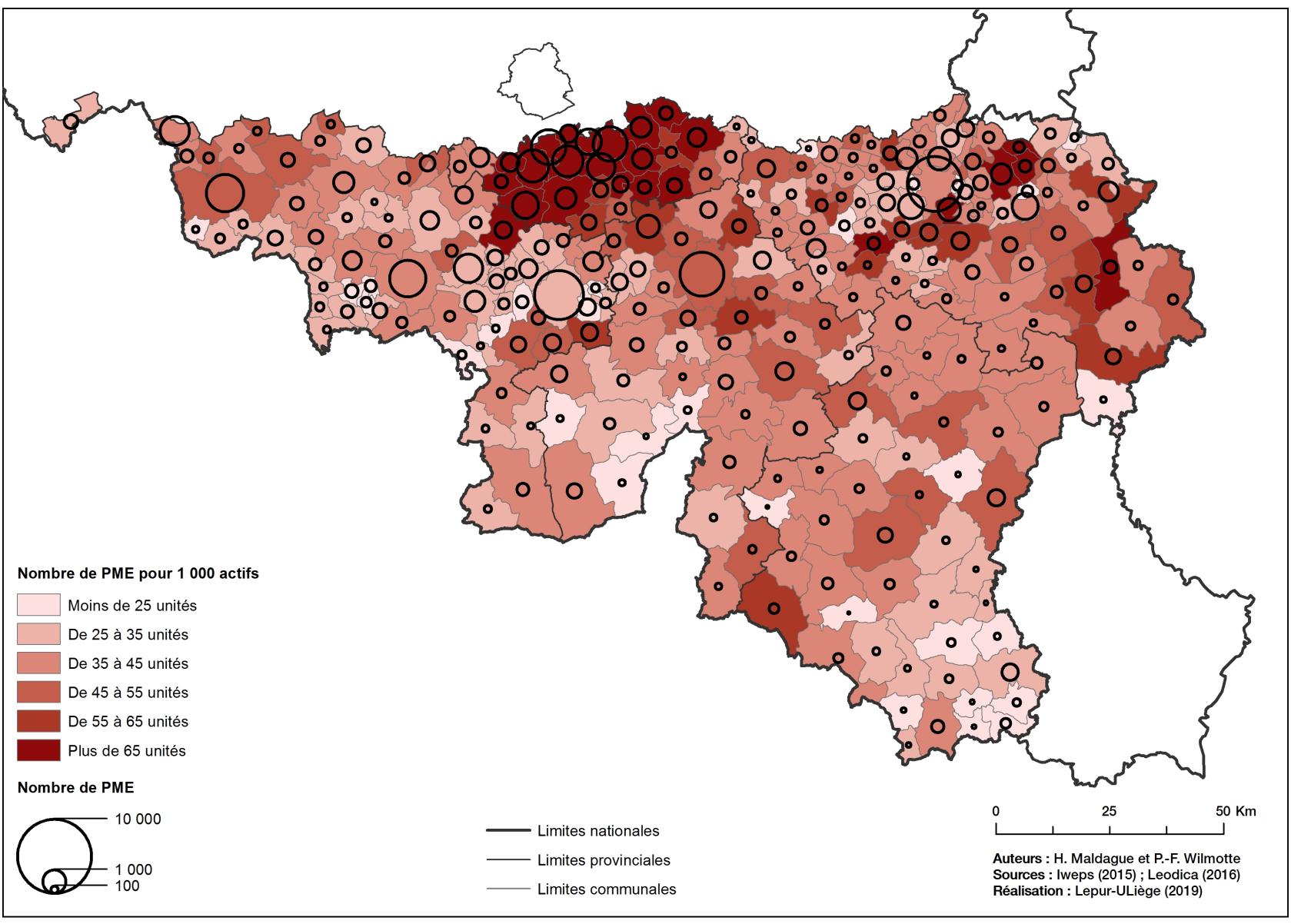

En nombres absolus, les plus grandes villes wallonnes concentrent les volumes les plus importants d'entreprises. Toutefois, si l'on tient compte du poids démographique, elles se positionnent à des niveaux moyens. Cela contraste avec la situation du Brabant wallon, qui représente en Wallonie une zone d'ampleur inégalée au niveau de l'intensité de l'entrepreneuriat. Cela résulte de la conjonction de nombreux facteurs, parmi lesquels le niveau d'éducation de la population active et la proximité spatiale aux marchés de la métropole bruxelloise.

Ailleurs en Wallonie, les communes les plus entrepreneuriales sont situées dans les zones périurbaines de Liège (dans le Condroz et surtout dans le Pays de Herve), de Namur et de Charleroi. Les communes périurbaines les plus entrepreneuriales accueillent généralement une population aisée, comme à Chaudfontaine, Neupré, Assesse ou Gerpinnes. Il est à noter que, à l'instar de nombreuses communes aisées du Brabant wallon situées aux portes de Bruxelles (Lasne, Waterloo, Rixensart, La Hulpe, etc.), on trouve dans ces entités périurbaines de nombreux indépendants qui, bien qu'exerçant leur activité dans la grande ville voisine, ont vraisemblablement établi le siège social de leur société à l'adresse de leur domicile. 
Des situations intermédiaires pour l'indice d'intensité de l'entrepreneuriat sont quant à elles observables dans les principaux pôles de la Wallonie picarde, mais aussi dans les petites villes situées au sud du sillon, à la condition toutefois qu'elles se situent à distance respectable de Luxembourg-Ville, cela depuis Bouillon jusque Waimes en passant par Libramont, Marche-en-Famenne, Bastogne et Saint-Vith.

Au final, la géographie de l'entrepreneuriat vérifie le rôle moteur des grandes villes. Cela concerne surtout Bruxelles, mais le phénomène est également observé à proximité des plus grandes villes wallonnes. Trois précisions sont toutefois à apporter à cette observation générale. Tout d'abord, l'effet des grandes villes ne se vérifie pas vis-à-vis de LuxembourgVille. Ensuite, force est aussi de constater que toutes les villes wallonnes ne génèrent pas le même potentiel d'activités. En effet, l'entrepreneuriat est bien plus développé dans les régions de Liège, de Namur ou même de Charleroi que dans les régions de Mons et de La Louvière. À Mons comme à La Louvière, ce constat s'applique tant aux communes périurbaines qu'aux communes centrales. Enfin, à l'image du logement, l'entrepreneuriat a également connu une périurbanisation. Même si la dynamique périurbaine est peut-être artificiellement gonflée par le fait que certains sièges sociaux soient localisés au lieu d'habitation du chef d'entreprise et non au lieu effectif d'activité, tout indique que les intensités les plus fortes en entrepreneuriat sont aujourd'hui le fait des périphéries aisées plutôt que des communes urbaines, qu'il s'agisse des communes de tradition industrielle ou même des communes centrales. Spatialement, l'entrepreneuriat devient donc très corrélé à l'attractivité résidentielle.

\subsection{LES SECTEURS PORTEURS POUR LE DÉVELOPPEMENT}

La troisième thématique que nous avons choisi de considérer afin de jauger du potentiel de développement futur de la Wallonie est celle des secteurs potentiellement les plus porteurs. Dans ce cadre, nous débutons par une sous-section sur les services supérieurs et, afin de fournir un éclairage spécifique sur la thématique du numérique, nous poursuivons avec une sous-section dédiée à la question des startups.

\subsubsection{Les services supérieurs}

L'état de l'art a souligné le rôle central des services aux entreprises sur le développement territorial, en particulier les services à forte intensité de connaissances qui sont susceptibles de soutenir la productivité et la compétitivité des firmes clientes. En anglais, ces services sont dénommés knowledge-intensive business services (KIBS). Afin d'approfondir cette thématique, nous avons réalisé la carte 22 en sélectionnant les KIBS suivants : les activités juridiques et comptables (NACE 69); les activités des sièges sociaux et de conseil en gestion (NACE 70) ; les activités d'architecture et d'ingénierie; les activités de contrôle et analyses techniques (NACE 71) ; les activités de recherche-développement scientifique (NACE 72) ; la publicité et les études de marché (NACE 73) ; les autres activités spécialisées, scientifiques et techniques (NACE 74) ; les activités vétérinaires (NACE 75). 
Carte 22. Répartition des emplois liés aux services supérieurs (2014)

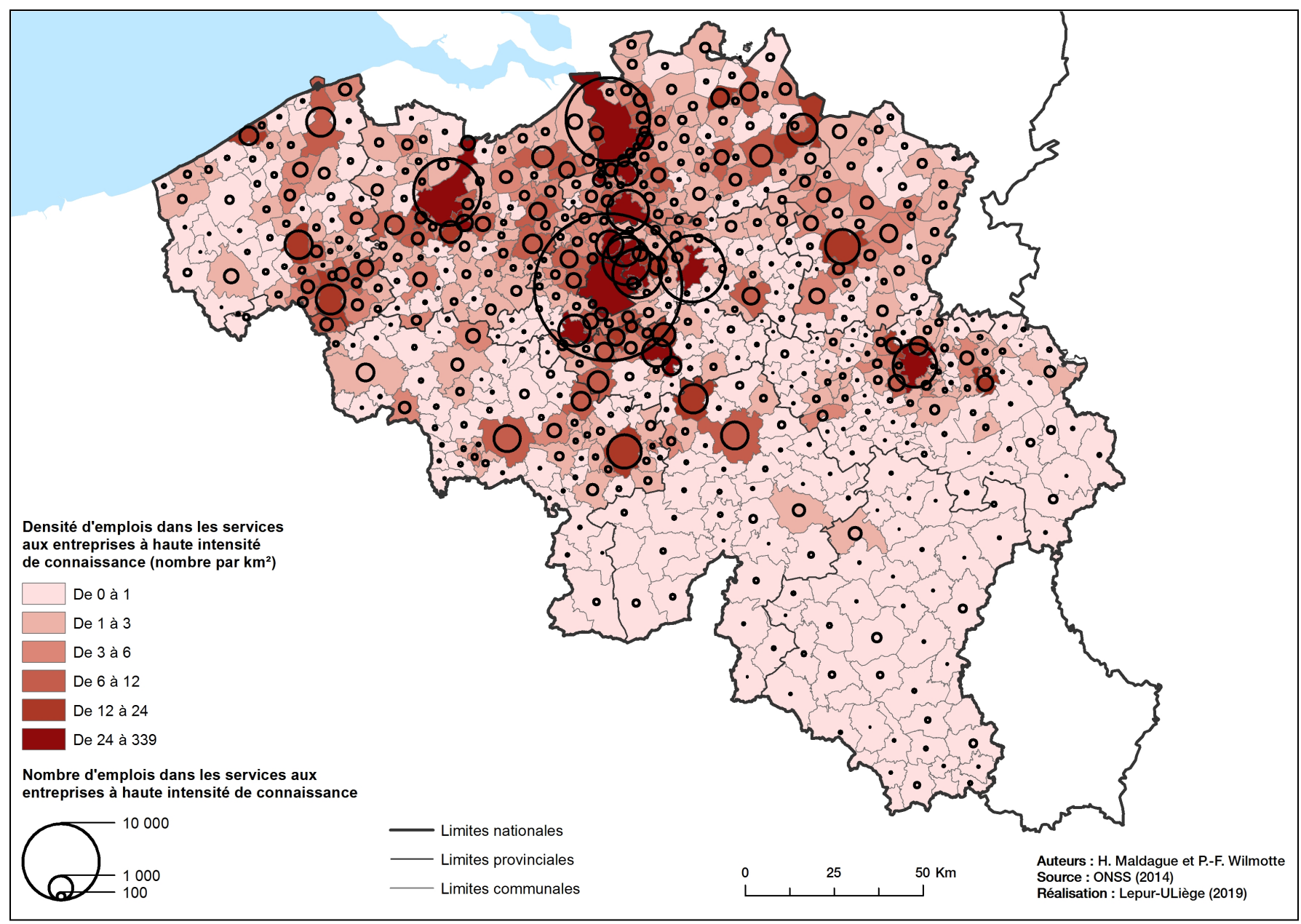

La carte 22 est à la fois peu surprenante et très interpellante. Elle confirme la faiblesse structurelle de l'économie wallonne vis-à-vis de la ressource territoriale des services spécialisés aux entreprises ${ }^{162}$. Elle confirme également que Bruxelles est le principal pôle belge pour les services spécialisés aux entreprises. Par Bruxelles, nous entendons ici les 19 communes de la région de Bruxelles-Capitale, mais également ses périphéries flamandes et wallonnes. Pour la partie flamande de la zone métropolitaine bruxelloise, les volumes d'emplois dans les services supérieurs sont particulièrement élevés à Zaventem et dans ses communes voisines, ainsi que dans les deux pôles secondaires de Louvain et de Malines. Derrière la zone métropolitaine de Bruxelles, on trouve ensuite deux autres concentrations importantes autour d'Anvers et de Gand.

En Wallonie, la répartition de l'emploi dans les services supérieurs reflète les constats émis pour les secteurs économiques précédemment analysés. Les villes du sillon - Mons, Charleroi, Namur et Liège - conservent un rôle polarisateur important mais, si l'on tient compte des poids démographiques, les pôles situés sous l'orbite bruxelloise apparaissent

162 M. BOURGEOIS et al., «Amélioration de l'attractivité et de la compétitivité du territoire wallon pour le secteur des services supérieurs », op. cit. 
plus dynamiques. Par rapport aux volumes d'emplois des communes bruxelloises et flamandes, ce dynamisme demeure toutefois limité, avec des volumes absolus qui, le long du sillon comme en Brabant wallon, correspondent à des activités comparables à des agglomérations flamandes de taille moyenne telles que Hasselt, Bruges, Courtrai ou Roulers. Pour la Wallonie, un autre fait marquant correspond à la quasi-absence de services supérieurs au sud du sillon. Cela rappelle combien les services supérieurs sont dépendants des économies d'agglomération.

Suite à une sollicitation du ministre-président wallon Paul Magnette (PS), la situation du maldéveloppement des services supérieurs en Wallonie a fait l'objet en 2015 d'une réflexion spécifique. Les principales conclusions de cette étude méritent d'être considérées afin de compléter notre propos.

Parmi ces conclusions, nous trouvons d'abord la vérification que l'économie wallonne est globalement affectée par la faiblesse de la ressource territoriale des services supérieurs. En effet, comme en témoigne le passage suivant, il est avéré par cette étude de 2015 que cette faiblesse a un impact sur la productivité et la compétitivité d'autres secteurs économiques: "Les acteurs interrogés reconnaissent tous que l'évolution des modes de production fait que le recours aux services aux entreprises peut contribuer de manière très significative à la performance des firmes industrielles et de services. Dans le même ordre d'idées, ils sont nombreux à souligner les carences de nombreuses PME wallonnes, par exemple en matière d'études de marché, de gestion informatique ou de maitrise des dispositifs fiscaux ${ }^{163}$.

Une autre conclusion est que la faiblesse des services supérieurs en Wallonie est illustrative d'une difficulté générale qui caractérise les régions de tradition industrielle. En effet, les grandes villes d'industrialisation ancienne comptent généralement moins d'emplois dans le tertiaire supérieur que leur taille ne le laisserait attendre ${ }^{164}$. Lors de l'état de l'art, nous avons constaté que la métropolisation résulte des avantages cumulatifs des grandes aires urbaines (cf. supra, 1.1.2). Avec les régions de tradition industrielle, nous sommes plutôt en présence de désavantages cumulatifs, qui limitent le développement de manière générale et le développement des services supérieurs en particulier. "Le faible développement des services supérieurs au sein des régions et des villes de tradition industrielle doit donc être appréhendé comme un processus cumulatif où se conjuguent des handicaps variés : des espaces et des mentalités façonnées par et pour l'industrie, des demandes trop faibles pour susciter l'émergence d'une offre locale, des représentations mentales négatives et des marchés immobiliers inefficaces ${ }^{165}$.

L'analyse spécifique menée en 2015 a montré que cette logique cumulative marque la Wallonie, où divers freins au développement des services supérieurs se renforcent suivant un principe de cercle vicieux. Il est ressorti de cette étude que ces principaux freins sont les suivants : la taille limitée des marchés wallons, la concurrence de territoires proches avec des niveaux d'attractivité plus élevés (en particulier Bruxelles et sa périphérie flamande), un manque d'intérêt pour les services supérieurs hérité de la culture industrielle, des politiques immobilières et d'urbanisme inadaptées (insuffisance des aménités

163 Ibidem, p. 46.

164 B. MÉRENNE-SCHOUMACKer et al., Les services supérieurs en Picardie, Amiens, CARMÉE, 2009.

65 M. BourgEOIS et al., "Amélioration de l'attractivité et de la compétitivité du territoire wallon pour le secteur des services supérieurs ", op. cit., p. 13. 
urbaines), un marché des services publics insuffisamment exploité, une Wallonie encore insuffisamment «business minded » et les faiblesses du capital humain wallon ${ }^{166}$.

\subsubsection{Les startups}

La startup peut être définie comme une entreprise susceptible, grâce à la création et à la commercialisation d'un produit digital, de développer une croissance qui ne va pas linéairement dépendre de son capital humain ${ }^{167}$. Nous prenons ici en compte le sujet des startups afin de compléter la question des secteurs porteurs et de fournir un éclairage complémentaire aux analyses réalisées à partir de la population de l'ensemble des entreprises situées en Wallonie (cf. supra, 6.2). Cet éclairage a été rendu possible grâce aux données disponibles via la plateforme startups.be.

Carte 23. Nombre de startups et d'institutions liées par commune (2019)

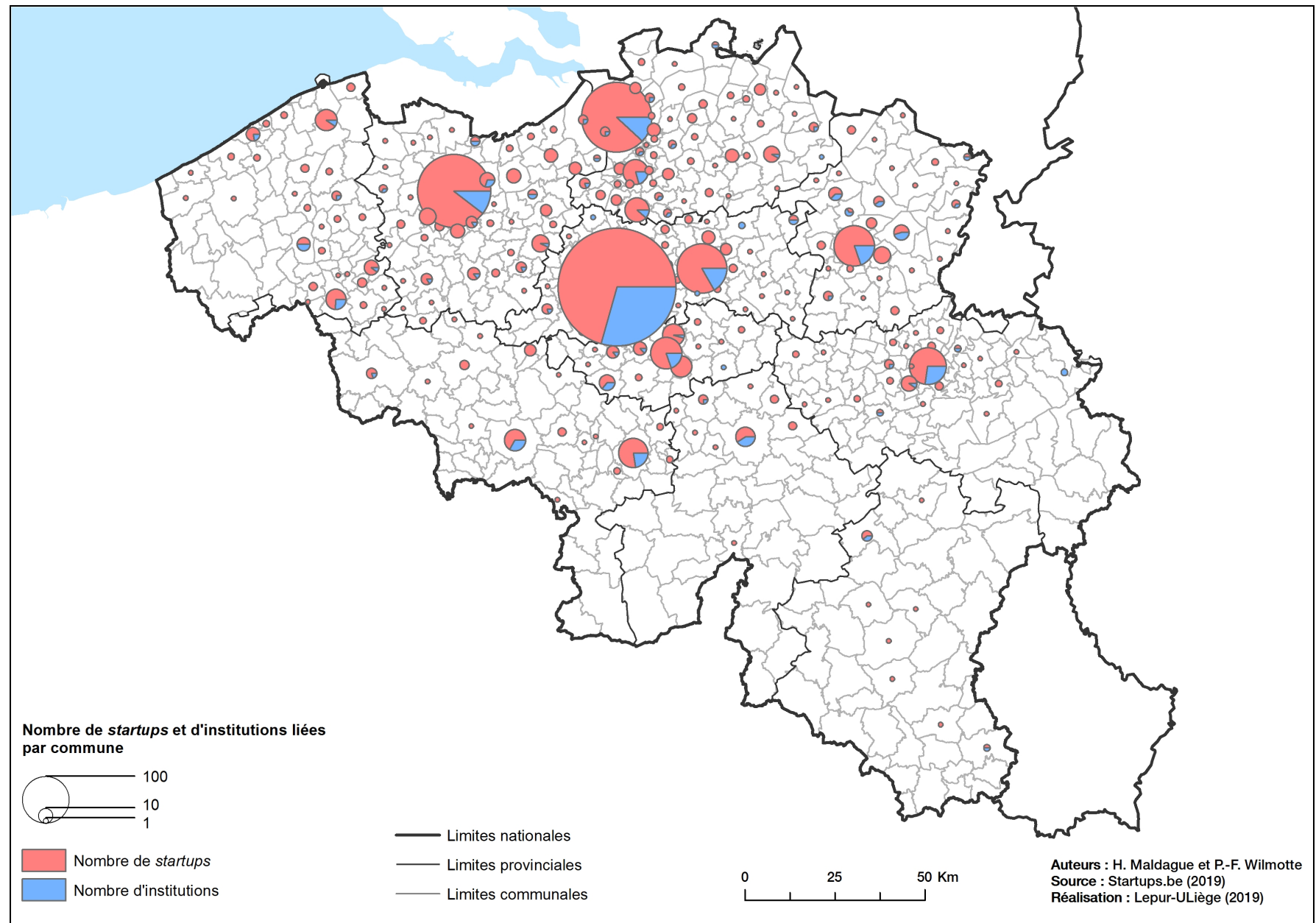

166 Le lecteur intéressé pourra aisément accéder à l'étude en question s'il souhaite approfondir ces constats et s'il souhaite prendre connaissance de recommandations diverses visant à améliorer l'attractivité et la compétitivité du territoire wallon pour ce secteur stratégique des services supérieurs aux entreprises.

167 O. MoHouT, « Making the shift from disruptive to hyper scalable», Sirris, s.d., www.sirris.be. 
La carte 23, établie à partir de cette source, porte sur le nombre de startups ainsi que sur le nombre d'institutions qui visent à faciliter leur création et leur développement. Ces institutions correspondent principalement à des centres de recherche, à des investisseurs technologiques et à des facilitateurs d'entrepreneuriat. Elle rend compte de la situation au $1^{\text {er }}$ juillet 2019.

Le premier constat qui ressort de la carte 23 est que, en comparaison de Bruxelles et des grandes villes flamandes, les pôles wallons ne représentent pas des catalyseurs aussi puissants pour l'entrepreneuriat lié aux startups. Il existe ici un différentiel nord-sud qui trouve son origine dans les causes structurelles invoquées ci-dessus à propos de la situation des services supérieurs. Par ailleurs, ce différentiel est vraisemblablement amplifié par le retard structurel de la société wallonne en matière de digitalisation ${ }^{168}$. Afin d'expliquer le retard productif de la Wallonie en matière de startups, il est vraisemblable qu'il faille également faire référence à un phénomène de transferts depuis la Wallonie vers Bruxelles ou vers l'étranger (à commencer par le Grand-Duché de Luxembourg). En effet, pour profiter d'un écosystème suffisamment structuré, l'entrepreneur wallon actif dans le secteur du numérique doit souvent quitter la région.

Il ressort aussi de la carte 23 que les implantations de startups et les écosystèmes qui les accompagnent sont, dans les différentes parties du pays, déterminés par des facteurs de localisation où les villes universitaires jouent un rôle déterminant. Pour la Wallonie, on recense ainsi approximativement 80 startups à proximité du campus brabançon de l'UCLouvain (principalement à Ottignies-Louvain-la-Neuve, Wavre et Mont-SaintGuibert) et une cinquantaine en région liégeoise. L'ouest du Brabant wallon (Brainel'Alleud et Nivelles), ainsi que les autres grandes villes wallonnes - Namur, Charleroi et Mons -, représentent des localisations secondaires, où l'on rencontre une dizaine de startups. En dehors de ces localisations, leur nombre est anecdotique.

En comparaison des autres types d'entreprises, la dynamique entrepreneuriale des startups apparaît moins soumise à une périurbanisation dispersée. Par exemple, en région liégeoise, les deux tiers des 50 startups recensées sont installées sur le territoire de la ville de Liège, en ce compris le site universitaire du Sart-Tilman et la partie la plus ancienne du Liège Science Park. La géographie de ces entreprises est en effet liée à celle des parcs scientifiques et autres parcs d'affaires. C'est ce qui explique la présence de telles entreprises au sein des communes de Marche-en-Famenne (Novalis Science Park), Gembloux (Parc scientifique de Crealys), Enghien (Qualitis Science Park), Nivelles (Les Portes de l'Europe), Seraing (Liège Science Park), Braine-l'Alleud (Parc de l'Alliance), Waterloo (Waterloo Office Park) ou Mont-Saint-Guibert (Axisparc).

À côté du rôle des parcs scientifiques et d'affaires, il existe également une tendance à la localisation des startups au sein des centres urbains des principales villes wallonnes. Ce phénomène de concentration spatiale est à mettre en lien avec les politiques urbaines en faveur de l'économie créative et culturelle (cf. supra, 1.2.2). En Wallonie, c'est dans le centre de Liège que l'application de ces politiques est la plus notable, en particulier avec l'action de Noshaq (anciennement Meusinvest) dans le développement d'un district créatif et d'un quartier numérique ${ }^{169}$.

168 J.-Y.HUWART, Pourquoi la Wallonie ne se redresse pas? Récit de quinze années de tentatives vaines, Namur, Éditions EG, 2019.

69 "Les nouveaux lieux d'innovation. Des accélérateurs économiques et de citoyenneté pour les villes ", Les cahiers Raisonnance. Cahier de réflexion des maires francophones, mai 2017, http://aimf.asso.fr. 
Afin de préciser le sujet des startups, il est opportun de se pencher sur la question des scale-ups, ces startups matures générant un volume important d'emplois et de valeurs ajoutées. Pour les scale-ups, la Belgique ne serait guère bien classée dans les comparaisons internationales ${ }^{170}$. En outre, les scale-ups belges sont très largement installées en Flandre (55\%) et à Bruxelles (35\%). Sur 40 scale-ups dans l'écosystème belge, seules quatre sont installées en Wallonie, en l'occurrence dans le Brabant wallon. De manière générale, les scale-ups sont situées à Bruxelles et à Gand et, dans une moindre mesure, à Anvers, à Louvain et dans le Brabant wallon. Au final, on voit donc que l'économie numérique et digitale se développe dans des lieux où l'on trouve de grandes universités et des pôles urbains attractifs, c'est-à-dire des lieux qui conjuguent économies d'agglomération et aménités urbaines.

170 O. Moноuт, European scale-up Report, Sirris, 2017. 


\section{PRINCIPAUX ENSEIGNEMENTS}

La présente livraison du Courrier hebdomadaire a débuté en rappelant que la question du déclin économique de la Wallonie est lancinante depuis des décennies. Afin d'approfondir ce sujet, nous avons mené une analyse de géographie économique sur la question des ressources territoriales, c'est-à-dire les caractéristiques des territoires qui permettent d'y attirer des flux de revenus. Dans ce dernier chapitre, nous présentons les principaux enseignements à retirer de notre travail.

\subsection{UNE TRAJECTOIRE DE REDRESSEMENT INSUFFISANTE}

Un premier enseignement de notre analyse est que la Wallonie n'est toujours pas positionnée sur une trajectoire de convergence vis-à-vis des régions voisines, la Flandre en particulier. Force est ici de diagnostiquer le décalage vis-à-vis des ambitions des décideurs wallons, pour lesquels la croissance du produit intérieur brut (PIB) est un préalable pour rencontrer les défis sociaux et environnementaux auxquels la région est confrontée. Nous aboutissons au diagnostic d'une trajectoire de redressement insuffisante sur la base de l'indicateur classique du PIB, mais également sur la base d'un indice composite établi en fonction d'objectifs formulés pour la stratégie Europe 2020. L'analyse liée à cet indice montre que, en dehors du Brabant wallon, la Wallonie peine à rencontrer les objectifs européens en matière de PIB, mais également en matière de taux d'emploi, de montants investis en recherche et développement (R\&D) ainsi que, dans une moindre mesure, en matière de formation et de capital humain.

La prise en compte du niveau de développement des régions européennes de tradition industrielle (RETI) conduit toutefois à nuancer le problème du maldéveloppement économique de la Wallonie. En effet, lorsque les indicateurs des différentes provinces wallonnes sont comparés aux indicateurs des régions de tradition industrielle étrangères, les résultats apparaissent globalement dans la moyenne. La Wallonie n'est donc pas la seule région qui peine à redéployer son économie. Face aux crises économiques, sociales et environnementales subies par les RETI, il n'existe pas de solution miracle. Toutefois, parmi le groupe des RETI, les faibles performances du Hainaut interpellent. De plus, les indicateurs de cette province se sont significativement dégradés entre 2010 et 2015 . Ce constat est à mettre en lien avec nos développements consacrés à l'ensemble territorial que nous dénommons la conurbation du Hainaut de tradition industrielle (cf. infra). 
L'analyse de l'indice composite confirme le constat bien connu qu'une des faiblesses structurelles de la Wallonie réside dans son taux d'emploi insuffisant. La réflexion menée à partir de l'indice composite a également mis en exergue la question de l'indicateur des sorties précoces du système scolaire. Même si cette variable s'est améliorée lors des dernières années, les niveaux de décrochage demeurent très élevés dans les provinces de Liège et de Hainaut, qui partagent cette faiblesse structurelle avec la région de BruxellesCapitale. Par contre, pour les provinces de Namur et de Luxembourg, plus rurales, le décrochage scolaire est moins problématique. Alors que le redéploiement économique de la Wallonie demeure pénalisé par les faibles performances de ses provinces de tradition industrielle, de telles observations rappellent combien l'efficacité et l'équité des systèmes scolaires sont fondamentales pour assurer le développement des territoires et des sociétés qu'ils hébergent.

Concernant la diplomation de l'enseignement supérieur et la part de la population appartenant à la classe créative, les indicateurs wallons sont relativement bons, même si les bassins de tradition industrielle continuent d'afficher des résultats peu flatteurs. En la matière, la situation de la Wallonie se rapproche de celle du Mezzogiorno, avec une production de richesse qui n'est pas au niveau attendu vis-à-vis des caractéristiques de la main-d'œuvre. Une explication tient au fait que l'activité professionnelle de nombreux Wallons qualifiés se situe en dehors du territoire régional. Toutefois, il est vraisemblable que ce décalage défavorable entre les caractéristiques de la main-d'œuvre et les niveaux de développement soit lié à d'autres facteurs variés et complexes, qui font en sorte que le potentiel du capital humain présent en Wallonie demeure insuffisamment valorisé pour la production locale de richesse.

La question lancinante du redéploiement économique est aussi à relier au constat du maldéveloppement des secteurs porteurs. En la matière, l'analyse conduit à diagnostiquer des logiques de handicaps cumulatifs et de cercles vicieux, où le maldéveloppement économique est à la fois la cause et la conséquence de la faiblesse de secteurs stratégiques tels que les services supérieurs et le numérique. À l'instar de l'étude initiée en 2015 par le ministre-président wallon sur les services supérieurs ${ }^{171}$, il apparait vital que les autorités wallonnes se penchent sur les conditions susceptibles de briser ces cercles vicieux.

\section{2. ÉCONOMIE PRODUCTIVE VERSUS ÉCONOMIE RÉSIDENTIELLE ET PRÉSENTIELLE}

La question du redéploiement économique de la Wallonie est structurellement indissociable des crises subies par ses bassins de tradition industrielle. De ce point de vue, la problématique du redéploiement est donc aussi structurellement indissociable de la question de l'économie productive, comme l'illustre l'indicateur du PIB par habitant, qui affiche en Wallonie une valeur globalement $30 \%$ inférieure à la moyenne belge ${ }^{172}$. En moyenne, la situation de l'économie résidentielle wallonne est meilleure. Pour

171 M. BourgEoIs et al., « Amélioration de l'attractivité et de la compétitivité du territoire wallon pour le secteur des services supérieurs ", op. cit.

C. AlbESSART et al., Rapport sur l'économie wallonne, op. cit., p. 271. 
l'indicateur du revenu par habitant, le décalage entre la moyenne nationale et la Wallonie n'est plus que de $\pm 10 \%$.

Le différentiel entre les deux indicateurs du PIB par habitant et du revenu par habitant résulte en grande partie des flux de travailleurs transfrontaliers. En effet, les Wallons se déplacent vers les entreprises puisque les entreprises ne se situent pas - ou plus - là où les Wallons résident. Bien que ce phénomène relativise la problématique du redéploiement économique de la Wallonie, il est toutefois impossible de s'en satisfaire. En effet, si le différentiel entre PIB et revenus s'explique par les navettes transfrontalières, il résulte également des transferts inter-régionaux induits par le système fiscal et le régime de sécurité sociale. Or, dans le contexte fédéral belge, des menaces lourdes pèsent sur les transferts inter-régionaux.

En outre, même si les navettes transfrontalières permettent de générer d'importants flux de revenus vers la Wallonie, différentes raisons plaident en faveur d'une situation où davantage de travailleurs wallons seraient occupés en Wallonie ${ }^{173}$. Primo, pour les autorités tant régionales que provinciales ou locales, il est budgétairement préférable que les travailleurs wallons soient occupés à l'intérieur des limites régionales. En effet, bien que les salaires des travailleurs résidant en Wallonie soient comptabilisés dans l'impôt des personnes physiques (IPP) wallon, diverses recettes fiscales échappent à la Wallonie et à ses collectivités territoriales lorsque l'activité est située en dehors de la région. Secundo, implanter davantage d'entreprises en Wallonie permettra un effet d'entraînement puisque ces nouvelles entreprises consommeront des biens et des services fournis par des firmes qui se trouvent à proximité immédiate. Tertio, si de nouvelles entreprises se créent dans les secteurs porteurs du numérique et des services supérieurs, cela pourrait également renforcer la compétitivité d'autres types d'activités. Nous pensons ici à un effet levier sur la compétitivité des petites et moyennes entreprises (PME), dont il apparaît qu'elles sont souvent mal armées en matière d'études de marché, de gestion informatique ou de maîtrise des dispositifs fiscaux. Quarto, le PIB par habitant demeurant l'indicateur principal de toute présentation de l'économie wallonne, la question de l'image de la région plaide également en faveur d'une occupation des Wallons en Wallonie. En effet, bien que la présentation en revenus soit moins défavorable, elle est moins courante et, en particulier, moins employée par la presse. Quinto, beaucoup de travailleurs wallons doivent actuellement parcourir de longues distances pour rejoindre un emploi situé à Bruxelles, en Flandre ou au Grand-Duché de Luxembourg, souvent d'ailleurs dans des conditions pénibles de congestion. En la matière, la création d'emplois en Wallonie même permettrait de réduire ce phénomène.

Si les difficultés économiques de la Wallonie sont d'abord liées à la restructuration de sa base productive, il ne faut toutefois pas négliger la question de la base résidentielle marchande. En effet, il ressort de nos analyses que la croissance potentielle de la Wallonie est pénalisée tant par un développement insuffisant du tourisme que par d'importantes concurrences frontalières dans le commerce de détail. Ce constat, qui s'appuie sur une comparaison avec les régions voisines, met en exergue la problématique de l'attractivité des villes et des campagnes wallonnes, pour les fréquentations touristiques comme pour les fréquentations commerciales.

173 M. BOURGEOIS et al., « Amélioration de l'attractivité et de la compétitivité du territoire wallon pour le secteur des services supérieurs », op. cit. 


\subsection{UNE WALLONIE PLURIELLE ET SEPT GRANDS ENSEMBLES TERRITORIAUX}

Le présent travail a permis de préciser le constat des disparités socio-spatiales qui caractérisent la Wallonie. Nos traitements effectués sur des indicateurs variés confirment que, en dépit de sa taille limitée, la géographie économique de la Wallonie est accidentée et son développement territorial pluriel. De ce point de vue, il n'existe pas un territoire wallon, mais des territoires wallons. Cela démontre que des analyses à partir d'indicateurs économiques établis à l'échelle régionale ne peuvent pas rendre compte de la complexité des grands enjeux du développement territorial. Des analyses géo-économiques multiéchelles sont dès lors nécessaires afin de prendre la mesure de la complexité des phénomènes et, en particulier, de la spatialité des externalités.

La très forte hétérogénéité qui marque le développement territorial en Wallonie pose question vis-à-vis d'une gouvernance généralement mise en œuvre de manière homogène sur l'ensemble de la région. Nous nous interrogeons donc ici quant à l'opportunité d'une démarche plus assumée de spécialisation des territoires ${ }^{174}$. Sur le terrain, qu'on le veuille ou non, les ressources territoriales fabriquent des territoires très différenciés. Face à cette différenciation ou spécialisation de fait, il ne semble pas crédible de tabler sur un développement homogène à l'échelle de la Wallonie; dès lors, il faut rechercher les complémentarités en affectant les moyens publics là où les effets de levier sont potentiellement les plus importants.

Concernant les aspects productifs, sept grands ensembles territoriaux aux limites floues et aux superpositions partielles se dégagent de nos travaux. Nous proposons la carte 24 afin de représenter ces ensembles qu'il nous semble opportun de distinguer pour décrypter la géographie économique de la Wallonie. Ils sont les suivants : le Triangle wallon ${ }^{175}$, la conurbation du Hainaut de tradition industrielle, la région liégeoise, la Wallonie picarde, l'Entre-Vesdre-et-Meuse, la Wallonie des faibles densités et le Sud-Luxembourg. En plus de ces sept ensembles territoriaux, la carte 24 identifie également divers pôles urbains extérieurs à la Wallonie ainsi que treize villes wallonnes dont la présence n'a d'autre but que d'aider à la bonne lecture de la carte. Parmi ces villes wallonnes, on trouve les douze villes éligibles à l'axe " développement urbain intégré " pour la programmation 2014-2020 de la politique de cohésion (ou douze villes « Feder») (cf. supra, 2.1.3.1) ainsi que le seul chef-lieu de province qui n'appartienne pas à cette catégorie, c'est-à-dire la ville de Wavre.

174 J.-Y. HuWART, Pourquoi la Wallonie ne se redresse pas?, op. cit.

175 Pour rappel, le Triangle wallon correspond à la zone d'influence de Bruxelles sur la Wallonie. Il est approximativement délimité par un triangle qui relie Bruxelles, Mons et Namur et dont la base passe par Charleroi ; il a pour cour le Brabant wallon. 
Carte 24. Grands ensembles territoriaux de l'économie productive

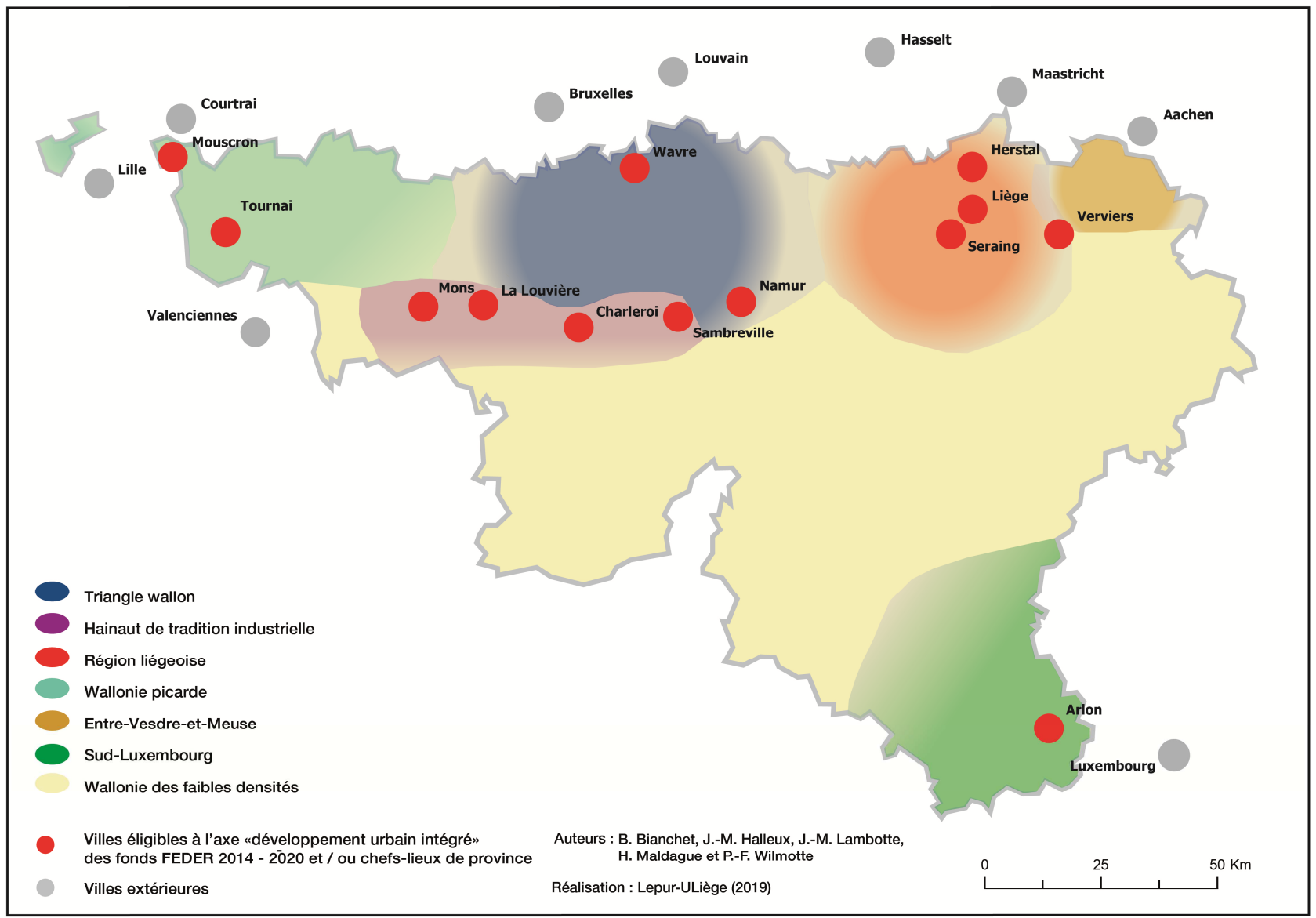

Parmi les sept grands ensembles territoriaux, le Triangle wallon est, du point de vue de l'économie productive, de loin le plus dynamique, avec en son cour le Brabant wallon. Grâce à son intégration dans la zone métropolitaine de Bruxelles, cette province bénéficie de puissantes économies d'agglomération qui, conjuguées à son attractivité résidentielle, en font un lieu où économie productive et économie résidentielle se renforcent mutuellement. En réalité, le Brabant wallon n'est pas seulement la province wallonne la plus dynamique, elle est aussi devenue la province belge où la croissance économique est la plus forte ${ }^{176}$. À l'instar de la périurbanisation bruxelloise, le dynamisme productif du Brabant wallon tend aujourd'hui à se diffuser, tant vers le Hainaut que vers la province de Namur, où de petites villes comme Soignies, Seneffe ou Gembloux assurent un rôle de relais pour l'économie résidentielle comme pour l'économie productive. À plus longue distance de Bruxelles, ce dynamisme trouve un relais mais aussi une limite au niveau de Namur.

Le deuxième ensemble spécifique est celui de la conurbation du Hainaut de tradition industrielle. Cet espace s'étend du Borinage jusqu'à l'est de l'agglomération carolorégienne, sur une cinquantaine de kilomètres de long et sur une vingtaine de kilomètres de large.

176 C. VANDERMOTTEN, «Les structures économiques de la Belgique et leur spatialité, des Golden Sixties à aujourd'hui ", Belgeo. Revue belge de géographie, n² 4, 2007, https://journals.openedition.org. 
Nous estimons son peuplement à plus de 700000 habitants, soit près de $20 \%$ de la population wallonne. Cette vaste zone pose problème en termes de ressources territoriales et, concomitamment, en termes de flux de revenus. Les faibles niveaux de revenus qui la caractérisent sont indissociables d'indicateurs défavorables pour la part de diplômés de l'enseignement supérieur, pour l'intensité de l'entrepreneuriat et pour les sorties précoces du système scolaire. Logiquement, les secteurs porteurs y sont très faiblement représentés, tant les services spécialisés aux entreprises que les startups du numérique.

Le niveau de développement de cet ensemble territorial apparaît d'autant plus interpellant qu'il semble englué dans un cercle vicieux de déficit d'attractivité et d'exode. Ce cercle vicieux met à mal les possibilités de développement des générations futures car, cohorte démographique après cohorte démographique, une part significative des jeunes, les plus dynamiques et les mieux formés, ont tendance à le quitter. Le diagnostic d'un cercle vicieux est à coupler à un diagnostic d'effets de lieu. Par effet de lieu, nous entendons le fait que, à caractéristiques individuelles égales, des personnes résidant dans un espace défavorisé et paupérisé seront susceptibles de rencontrer plus de difficultés dans leurs parcours scolaires et professionnels. S'agissant du cas étudié, nous sommes vraisemblablement en présence de populations pour lesquelles les probabilités de réussir des études supérieures ou de devenir entrepreneur sont réduites vu le déficit en stimuli et en effets d'entraînement. A contrario, les probabilités de connaître une sortie précoce du système scolaire sont augmentées, ce qui est souvent synonyme de chômage et d'une reproduction intergénérationnelle de la précarité. Cette hypothèse de la prégnance d'effets de lieu apparait d'autant plus vraisemblable que, en matière d'enseignement, il est bien connu que la logique des externalités entre élèves plaide en faveur de la mixité sociale et des mélanges d'enfants divers dans les mêmes classes ${ }^{177}$.

Au sein de cette vaste conurbation du Hainaut de tradition industrielle, il convient toutefois d'établir une distinction entre les communes périphériques de tradition industrielle et les villes centrales de Mons et de Charleroi, où les économies d'agglomération et les ressources territoriales sont plus développées. Pour Mons et ses communes limitrophes (hors Borinage), une ressource territoriale potentielle correspond aux niveaux de qualification relativement élevés. Pour Charleroi, les statistiques sont moins flatteuses pour les niveaux de formation, mais elles sont meilleures pour la base productive. Par exemple, comme l'illustrent les géographies des grandes entreprises et des membres des pôles de compétitivité, Charleroi demeure un pôle technologique important (grâce notamment au Biopark, à Alstom et à la Société anonyme belge de constructions aéronautiques - SABCA). Plutôt qu'une dynamique purement carolorégienne, ce positionnement est indissociable de la dynamique métropolitaine bruxelloise. Spatialement, le nord de Charleroi correspond ainsi à un espace de chevauchement entre les dynamiques vertueuses du Triangle wallon et la faible attractivité du Hainaut de tradition industrielle.

Le troisième ensemble qui se dégage est celui de la région liégeoise. On y trouve de fortes ségrégations socio-spatiales qui, sur un territoire peu étendu, opposent des lieux qui

177 H. SELOD, « La mixité économique et sociale », in J.-F. THISSE et al. (dir.), Ville et économie, Paris, La Documentation française, 2004, p. 129-156; C. DUJARDIN, H. SELOD, I. THOMAS, « Residential segregation and unemployment: the case of Brussels ", Urban Studies, volume 45, n 1, 2008, p. 89-113 ; N. RYELANDT, "Les décrets "inscriptions" et "mixité sociale" de la Communauté française », Courrier hebdomadaire, CRISP, $\mathrm{n}^{\circ}$ 2188-2189, 2013. 
s'apparentent tantôt à la conurbation hennuyère et tantôt au cœur du Triangle wallon. Avec ses communes périphériques de tradition industrielle, la ville de Liège forme un ensemble de plus de 350000 personnes. Cet ensemble présente certaines caractéristiques comparables à la conurbation du Hainaut de tradition industrielle. Les géographies des revenus, des diplômés de l'enseignement supérieur et du dynamisme entrepreneurial nous obligent, ici aussi, à diagnostiquer des effets de lieu et un cercle vicieux de déficit d'attractivité.

Corollairement, la région liégeoise dispose d'atouts productifs non négligeables, grâce à la taille de son bassin d'emploi et à ses infrastructures, mais aussi grâce à ses aménités urbaines et aux niveaux de formation relativement élevés induits par son offre en enseignement supérieur. Ces ressources territoriales permettent d'expliquer pourquoi plusieurs communes de la périphérie sud affichent des intensités entrepreneuriales élevées. Elles permettent aussi d'expliquer pourquoi le bassin liégeois représente une polarité majeure au niveau de la géographie des pôles de compétitivité (cf. supra, 4.1.4). En outre, bien que le phénomène demeure modeste en comparaison des dynamiques bruxelloises et flamandes, la partie centrale de l'agglomération liégeoise parvient à émerger dans le milieu de l'économie digitale et créative.

Bien que situés à deux extrémités géographiques de la Wallonie, le quatrième et le cinquième ensembles présentent des similitudes. Il s'agit de la Wallonie picarde et de l'Entre-Vesdre-et-Meuse (y compris le nord de la Communauté germanophone). Ces deux zones sont spécialisées dans les secteurs de l'agro-alimentaire et de la distributionlogistique, comme en témoigne leur représentation dans les pôles de compétitivité Wagralim et Logistics in Wallonia. Vis-à-vis des territoires voisins, les deux ensembles se caractérisent par une population à la culture entrepreneuriale plus développée. Même si les économies d'agglomération y sont moins prégnantes qu'en Brabant wallon ou qu'en région liégeoise, il en résulte des bases productives étoffées. Il s'agit là d'une observation encourageante qui montre que, en Wallonie comme ailleurs, de bonnes performances économiques demeurent possibles en dehors des espaces les plus métropolisés ${ }^{178}$.

À l'inverse de la Wallonie picarde et de l'Entre-Vesdre-et-Meuse, l'économie productive est amorphe dans le Sud-Luxembourg. Cette déficience de l'entrepreneuriat est vraisemblablement induite par les avantages fiscaux que le Grand-Duché voisin propose aux entreprises et aux travailleurs, avec un véritable effet " aspirateur » à la création d'entreprises. Il en résulte une configuration singulière avec, d'un côté, une économie productive aux caractéristiques similaires aux parties les moins dynamiques du Hainaut et, d'un autre côté, des niveaux de revenus médians et de diplomation de l'enseignement supérieur proches du Brabant wallon.

Une vaste partie du territoire régional est à intégrer dans l'ensemble de la Wallonie des faibles densités, avec des densités de population inférieures à 150, voire à 100 ou 50 habitants par kilomètre carré. La majeure partie de la Wallonie des faibles densités est située au sud du sillon Sambre-et-Meuse, depuis la Botte du Hainaut jusqu'aux communes de Butgenbach et Bullange en région de langue allemande. Vers le sud, la Wallonie des faibles densités s'étend sur la majeure partie de l'Ardenne. On y trouve les communes les plus rurales de Wallonie. Nos analyses montrent que les ressources territoriales y sont

178 O. Bouba-Olga, M. GRossetTi, La mythologie CAME (Compétitivité, attractivité, métropolisation, excellence): comment s'en désintoxiquer?, HAL, 2018, https://hal.archives-ouvertes.fr. 
faibles et que les revenus y dépendent plus qu'ailleurs du tourisme. Nos analyses montrent également que l'attractivité pour le secteur de la logistique y est réduite vu l'accessibilité moyenne aux marchés européens. Concernant cette partie du territoire wallon, nos indicateurs mettent donc à mal la thèse d'un redéploiement wallon qui pourrait structurellement s'appuyer sur une densification des pôles situés le long de l'axe de la Nationale 4.

La Wallonie des faibles densités ne correspond toutefois en rien à une "Wallonie du vide », qui serait dépourvue de dynamisme économique local. Rappelons ici que l'analyse de l'emploi marchand a permis d'identifier certaines polarités où l'on trouve une orientation productive affirmée, par exemple Marche-en-Famenne ou Libramont-Chevigny (cf. supra, 4.1.2). Toutefois, force est de constater que l'activité induite par ces petites villes n'est pas suffisante pour générer un impact significatif à l'échelle régionale. Sur un plan plus qualitatif, force est ici de constater que la faiblesse des ressources territoriales s'y accompagne d'un développement marginal des secteurs les plus porteurs, comme l'illustrent les analyses dédiées aux pôles de compétitivité, aux services supérieurs ou aux entreprises éligibles au FEDER ${ }^{179}$.

\subsection{DÉVELOPPEMENT TERRITORIAL ET MÉTROPOLISATION}

Notre état de l'art a mis en exergue le rôle des grandes villes dans le développement des territoires. Ce rôle est lié aux économies d'agglomération et, en particulier, à l'économie d'agglomération de l'accès à un vaste marché du travail partagé. Dans un contexte où l'économie de la connaissance dépend grandement des réserves disponibles en maind'œuvre qualifiée, la taille des marchés de l'emploi est d'une importance critique pour la métropolisation de l'économie (cf. supra, 1.1.2).

En Wallonie, la proportionnalité entre niveaux de développement et tailles des bassins d'emplois n'est que partiellement vérifiée. D'un côté, la vérification est effective pour le Triangle wallon (dont le Brabant wallon). En effet, le dynamisme de cette zone est la résultante de son intégration dans la vaste zone métropolitaine articulée par Bruxelles. La zone de recrutement du pôle d'emploi de Bruxelles représente un marché de l'emploi estimé par l'Organisation de coopération et de développement économiques (OCDE) à plus de 2,6 millions d'habitants ${ }^{180}$. En y ajoutant les autres aires urbaines qui constituent l'espace Europolis - c'est-à-dire la somme du Triangle wallon et du Diamant flamand le volume de population y dépasse les 6 millions d'habitants.

\footnotetext{
179 Afin d'illustrer la difficulté de développer des activités à hautes valeurs ajoutées dans des zones où les économies d'agglomération sont faibles, nous pouvons nous appuyer sur l'exemple de l'organisation du groupe de construction Thomas \& Piron. Il s'agit de la principale entreprise wallonne du secteur de la construction, dont l'activité a débuté à Our, un village ardennais. Les activités traditionnelles du groupe (Thomas \& Piron Home) y trouvent toujours leur siège central. Par contre, les nouvelles activités liées au développement de projets urbains de grande envergure (Thomas \& Piron Bâtiment) sont implantées à proximité de Namur, qui bénéficie d'une meilleure accessibilité et d'un accès plus aisé à la maind'œuvre qualifiée et pluridisciplinaire que nécessitent l'élaboration et la réalisation de projets urbains.

180 Nous faisons ici référence au travail de l'OCDE sur la délimitation des régions urbaines fonctionnelles. À ce propos, cf. OCDE, « Functional urban areas. Belgium », janvier 2019, https://www.oecd.org.
} 
En revanche, la proportionnalité entre niveaux de développement et tailles des aires urbaines n'est pas vérifiée pour les cas de Liège et de Charleroi, qui ne correspondent toujours qu'à des métropoles potentielles. Selon l'OCDE, leurs bassins d'emploi respectifs correspondent à \pm 750000 et \pm 500000 habitants. Parmi les villes wallonnes, seuls ces deux pôles disposent donc d'une masse critique suffisante au déploiement d'une dynamique métropolitaine. Pour Namur et Mons, les volumes démographiques sont bien moindres, avec des bassins d'emplois de \pm 200000 habitants.

Afin d'expliquer pourquoi le potentiel démographique de Liège et de Charleroi n'induit pas le développement attendu, il faut bien sûr invoquer les crises des secteurs industriels dans lesquels ces régions s'étaient spécialisées depuis le XIX ${ }^{e}$ siècle et, surtout, l'impact de ces crises sur les caractéristiques à la fois territoriales et sociales des bassins. Rappelons ici le cercle vicieux du déficit d'attractivité, cercle vicieux qui doit être mis en lien avec l'impact de la désindustrialisation sur la dégradation physique des espaces. Rappelons aussi les effets de lieu induits par la concentration spatiale des difficultés sociales. Par ces effets de lieu, nous sommes en présence, non pas d'externalités positives et d'économies d'agglomération, mais d'externalités négatives et de spirales descendantes qui amplifient les problèmes économiques et sociaux.

Au regard de leur potentiel démographique, mobiliser des moyens afin de soutenir le redéploiement et la métropolisation effective de Charleroi et de Liège se justifie d'abord pour des raisons d'efficacité économique. Cette proposition n'est pas neuve et elle a par exemple été formulée voici plus de dix ans par Jacques-François Thisse et Isabelle Thomas : «En dehors de l'axe Bruxelles-Namur, ces deux villes sont celles, pourtant, qu'il faut promouvoir du fait de la faiblesse de l'armature urbaine wallonne ${ }^{181}$. En réalité, les bassins de Charleroi et de Liège ont été largement soutenus depuis que la Wallonie dispose de ses propres instruments économiques ${ }^{182}$. Toutefois, historiquement, l'essentiel de ce soutien a été consacré au maintien d'activités économiquement peu viables mais socialement sensibles. Aujourd'hui, à l'heure du renouvellement progressif de leurs bases productives, les problèmes structurels de ces bassins résultent moins de crises industrielles contemporaines que de l'héritage des crises passées. L'écueil d'un soutien à des activités peu viables est dès lors moins menaçant aujourd'hui que lors des " errements " ${ }^{183}$ de la mise en œuvre de la politique wallonne en faveur du redéploiement régional.

En plus de raisons liées à l'efficacité économique, le soutien à Charleroi et à Liège se justifie également pour des raisons liées à l'équité sociale. Au sein de nombreux États et régions, mobiliser des moyens pour aider les plus grandes villes à se positionner dans la concurrence métropolitaine se traduit par un renforcement des déséquilibres spatiaux vis-à-vis des territoires en retard de développement ${ }^{184}$. La Wallonie s'écartant du modèle général entre niveaux de développement et tailles des aires urbaines, elle n’est actuellement pas concernée par cet écueil.

181 J.-F. Thisse, I. ThOMAS, « Bruxelles et Wallonie », op. cit., p. 92.

182 P. Delforge, « Politique économique wallonne (1971-2011): de Freddy Terwagne à Marshall 2.Vert, ou comment retrouver la prospérité d'antan? », in M. VAN DEN WIINGAERT (dir.), D'une Belgique unitaire à une Belgique fédérale. Quarante ans d'évolution politique des Communautés et des Régions (1971-2011), Bruxelles, ASP, 2011, p. 149-161.

183 H. CAPRON, «Croissance et développement spatial inégal des régions », op. cit.

184 O. Bouba-Olga, M. GrossetTi, La mythologie CAME, op. cit. 


\section{VERS UNE SPÉCIALISATION DES TERRITOIRES : DES POLITIQUES DE DÉVELOPPEMENT À MIEUX ADAPTER AUX CONFIGURATIONS TERRITORIALES}

En plus de chercher à mieux comprendre la géographie économique du territoire wallon, un autre objectif du présent Courrier hebdomadaire a été de faire le point sur les politiques publiques en matière de développement économique et territorial. Cela pousse maintenant à s'interroger sur l'opportunité d'une spatialisation et spécialisation plus détaillée de ces politiques, en les ajustant plus finement aux forces et faiblesses des ensembles territoriaux décrits ci-dessus.

Pour le Triangle wallon (et le Brabant wallon en particulier), de nombreux indicateurs sont au vert, notamment les niveaux de formation et les cultures entrepreneuriales. $\mathrm{Au}$ regard de l'ensemble des analyses, le Brabant wallon affiche toutefois des résultats moins positifs pour les services spécialisés aux entreprises, qui n'y trouvent pas (encore) l'ensemble des ressources territoriales qu'ils recherchent, en particulier les aménités urbaines. Par ailleurs, une autre faiblesse du Brabant wallon est celle d'une croissance économique qui prend trop souvent la forme d'une périurbanisation diffuse. Face à ces constats, il apparait pertinent de mieux limiter la périurbanisation et d'initier des projets urbains ${ }^{185}$ susceptibles de renforcer et de structurer les centres les plus importants. Nous pensons tout d'abord à la polarité de Wavre-Ottignies-Louvain-la-Neuve, qui est susceptible d'atteindre une attractivité comparable à celle des pôles secondaires de l'hinterland bruxellois que sont Louvain et Malines. Nous pensons également aux deux polarités de Braine-l'Alleud-Waterloo, d'une part, et de Nivelles, d'autre part. En renforçant et en structurant ces trois pôles principaux, la partie wallonne du territoire sous l'orbite bruxelloise serait susceptible d'absorber une part plus importante des développements métropolitains et, en particulier, des croissances du secteur des services supérieurs.

Pour la conurbation du Hainaut de tradition industrielle, au vu des structures sociales héritées des déclins industriels, il ne paraît pas réaliste d'espérer un redressement à court ou même à moyen terme (une génération). Un redressement sur le moyen-long terme (plusieurs générations) est envisageable, mais à la condition d'y briser les cercles vicieux du déficit d'attractivité et d'y atténuer les effets de lieu responsables de la reproduction intergénérationnelle de la précarité. Dans cette perspective, la stratégie du soutien à l'économie résidentielle apparaît sans doute préférable à la stratégie du soutien à l'économie productive. En d'autres termes, dans ces territoires en crise rémanente, il semble plus opportun d'y aider les personnes que d'y aider les entreprises. Cela pourrait passer par des discriminations positives visant à y améliorer les environnements résidentiels, avec la perspective d'y renforcer la mixité sociale et d'y limiter la fuite des jeunes les plus à même de reconstruire le tissu économique. En parallèle, tout doit être fait pour y améliorer la performance des systèmes scolaires, afin de réduire les sorties précoces et afin de renforcer l'accès à l'enseignement supérieur (en particulier dans les bassins de Charleroi et de La Louvière). La stratégie de l'économie présentielle au sein

185 N. ARAB, « Pour une théorie du projet en urbanisme », Revue européenne des sciences sociales, volume 56, $\mathrm{n}^{\circ} 1,2018$, p. 219-240. 
des communes hennuyères de tradition industrielle se justifie également pour des raisons de transition écologique et d'aménagement du territoire. Comme il s'agit de territoires relativement denses, y renforcer le peuplement permettrait de limiter la dépendance à l'automobile et tous les problèmes associés ${ }^{186}$.

Pour le Hainaut de tradition industrielle, l'option de privilégier l'économie résidentielle plutôt que l'économie productive paraît d'autant plus justifiée que, sur la base des traitements réalisés, de nombreuses localités n'y disposent pas - ou n’y disposent plus, vu leur passé industriel - des ressources territoriales qui permettent aux entreprises de demeurer compétitives. Par rapport à l'idée de privilégier l'économie résidentielle plutôt que l'économie productive, il apparaît donc légitime de s'interroger sur le choix de la programmation FEDER 2014-2020 de mobiliser des moyens importants pour l'aide à l'investissement à l'échelle de l'ensemble du Hainaut. En outre, cette orientation stratégique tend à favoriser une forte périurbanisation des entreprises et un sousinvestissement dans les tissus urbains, où il subsiste pourtant de nombreuses friches. Par ailleurs, ces réflexions sur les aides publiques renforcent les interrogations quant à l'efficience du dispositif des zones franches, en tout cas tel qu'il est appliqué en Wallonie. Pour rappel, dans cette zone du Hainaut de tradition industrielle, les autorités wallonnes ont choisi d'utiliser ce dispositif en sélectionnant deux sites hennuyers (Doosan à Frameries et Caterpillar à Gosselies) ainsi que le site voisin de Saint-Gobain Sekurit à Sambreville.

S'il s'agit d'aider les entreprises afin de contribuer au développement de la base productive, il semble important d'être sélectif, sur le plan sectoriel, à l'image des pôles de compétitivité, mais aussi sur le plan spatial. Dans la conurbation du Hainaut de tradition industrielle, la sélectivité spatiale justifie de miser sur les deux grands pôles de Mons et de Charleroi. $\mathrm{Vu}$ les volumes de population qu'ils concentrent, on peut penser que leurs économies d'agglomération permettent d'y assurer le développement de l'économie productive. Dans le champ de l'économie productive, centraliser les moyens sur les lieux qui concentrent les populations et les activités conduit donc à renforcer les effets de levier, c'est-à-dire à optimaliser l'efficacité des politiques publiques.

En région liégeoise, les communes paupérisées semblent devoir être aidées sur le même modèle que dans le Hainaut de tradition industrielle, afin d'y renforcer l'attractivité et afin d'y réduire les effets de lieu. Par ailleurs, après le Brabant wallon, Liège est la deuxième zone de Wallonie en termes d'économies d'agglomération, comme l'illustrent notamment les développements dans l'aéronautique et dans les biotechnologies. Pour Liège, les économies d'agglomération tiennent à la taille du bassin d'emplois, aux niveaux de formation des populations et également aux aménités urbaines. En conséquence, l'économie productive doit également y être soutenue, dans la perspective de renforcer la métropolisation émergente qui caractérise la ville centrale et certaines de ses périphéries.

Pour les deux ensembles de la Wallonie picarde et de l'Entre-Vesdre-et-Meuse, l'intérêt des pôles de compétitivité Wagralim et Logistics in Wallonia est à souligner. Selon nos travaux sur l'organisation spatiale des pôles, les appels à projets successifs semblent avoir contribué à renforcer l'intégration de ces deux régions au reste de l'économie wallonne. Il parait fondamental de poursuivre dans cette voie, afin que la culture entrepreneuriale

186 C. BAZET-SimOni et al., « Face à l'épuisement du pétrole, quel rôle pour l'aménagement du territoire en Wallonie? ", op. cit. 
qui les caractérise puisse être soutenue par les institutions en $R \& D$ du Triangle wallon et de la région liégeoise.

Nous avons constaté que la Wallonie des faibles densités repose essentiellement sur une économie présentielle. Ce constat pousse à s'interroger sur les modalités qui permettraient d'y soutenir efficacement l'économie productive. Il s'agit là d'une question qui se pose de la même manière dans de nombreux territoires à la fois peu dotés en économies d'agglomération et peu accessibles. Force est ici de constater que la littérature scientifique n'y apporte pas réellement de réponses ${ }^{187}$. Pour ces territoires périphériques, la croissance de l'économie résidentielle et présentielle offre vraisemblablement de meilleures perspectives de développement que l'économie productive. En particulier, vu la faiblesse des densités et des économies d'agglomération, il ne semble pas opportun de mobiliser des moyens publics afin d'y initier des projets de type métropolitain. En effet, tout indique que les résultats attendus ne seront pas au rendez-vous, comme l'illustrent divers projets de centres de congrès ou de parcs d'activités dédiés à la logistique ou à des entreprises à forte composante technologique. Plutôt que d'inutilement chercher à imiter les logiques métropolitaines, ces territoires ont donc intérêt à identifier des avantages différenciatifs qui permettraient de soutenir des domaines susceptibles de bénéficier d'une «innovation décentralisée ${ }^{188}$ tels que les productions liées au terroir (pratiques agricoles), à la production énergétique ou aux ressources locales (pierre, terre, bois).

Pour l'ensemble territorial du Sud-Luxembourg, une amélioration significative de la situation vis-à-vis de l'atonie productive ne peut provenir que d'une réflexion collaborative menée à l'échelle de la zone métropolitaine luxembourgeoise. Aussi longtemps que les différentiels fiscaux entre la Belgique et le Grand-Duché de Luxembourg seront tels, il ne semble pas réaliste d'attendre une amélioration de la situation. Si cette réflexion collaborative avec les autorités grand-ducales ne peut aboutir, sans doute faudra-t-il alors chercher à préciser les modalités à appliquer afin de tirer le meilleur parti d'un rôle de périphérie purement résidentielle.

\subsection{DES RESSOURCES TERRITORIALES À LA GÉOPOLITIQUE TRANSFRONTALIÈRE : UN CONTEXTE AMBIVALENT DE CONCURRENCE ET DE COOPÉRATION AVEC LES RÉGIONS VOISINES}

La dimension géopolitique est manifeste dans la zone métropolitaine luxembourgeoise, mais elle l'est également dans la zone métropolitaine bruxelloise, où il est vital d'éviter la concurrence frontale dans un contexte institutionnel poly-régional et polycommunautaire. Une multiplication des coopérations s'impose dès lors, afin de tirer le meilleur parti des économies d'agglomération dont bénéfice l'espace Europolis, tout

187 B. PECQUEUR, «Esquisse d'une économie territoriale », L'Espace géographique, volume 43, n 3, 2014, p. 198-214.

B. GUESNIER, "Décentralisation et innovation : le rôle central du "territoire" ", Marché et organisations, $\mathrm{n}^{\circ} 25,2016$, p. $29-43$. 
en évitant d'y renforcer les grands problèmes auxquels il est confronté, en particulier en termes d'étalement urbain et de mobilité.

Afin de profiter pleinement du potentiel bruxellois, la Wallonie devra collaborer tant avec la Flandre qu'avec Bruxelles, mais elle devra également encadrer et canaliser les développements qui s'opéreront sur son propre territoire. En effet, le risque est grand que la croissance économique du Triangle wallon ne soit entravée si la croissance métropolitaine continue de se matérialiser par une périurbanisation diffuse. Dès lors, il serait essentiel de mobiliser le savoir-faire des urbanistes et des aménageurs en vue d'améliorer les aménités urbaines dans les trois zones principales de développement que sont Wavre-Ottignies-Louvain-la-Neuve, Braine-l'Alleud-Waterloo et Nivelles (cf. supra).

Comme nous venons de le voir tant à propos du Sud-Luxembourg qu'à propos de l'espace Europolis, la géopolitique transfrontalière est étroitement liée à la question de la métropolisation. La Wallonie gagnerait ici à stimuler la prospection d'acteurs transfrontaliers susceptibles de renforcer les secteurs sur lesquels elle souhaite s'appuyer et, en particulier, des acteurs qui ont été capables de développer des compétences de pointe. Cette proposition vaut pour les quatre aires métropolitaines transfrontalières décrites au chapitre 5. Précisons que la plupart des pôles de compétitivité wallons recouvrent des secteurs ayant également été reconnus dans les territoires voisins comme des secteurs stratégiques ${ }^{189}$. En effet, à l'instar de la Wallonie avec ses clusters et ses pôles de compétitivité, les autorités des régions voisines se sont également dotées d'outils visant à renforcer l'innovation par la stimulation des liens entre entreprises et centres de recherche ${ }^{190}$. Rappelons sur ce sujet le constat interpellant de la non-intégration des acteurs liégeois dans le projet Top-Technology Region Eindhoven-Leuven-Aachen Triangle (TTR-ELAt) (cf. supra, 5.2.3).

\subsection{DES QUESTIONNEMENTS EN SUSPENS ET DES BESOINS CRIANTS EN DONNÉES ET EN RECHERCHES}

À l'issue de notre recherche, force est de constater que de nombreux questionnements demeurent en suspens. Sans aucun doute, un questionnement qui mériterait d'être approfondi est celui de l'efficacité des politiques publiques mises en œuvre pour redresser l'économie wallonne. Nous pensons en particulier à la politique emblématique des pôles de compétitivité qui, en raison de grandes difficultés méthodologiques et en dépit des efforts de l'Institut wallon de l'évaluation, de la prospective et de l'économie (IWEPS), demeure difficile à évaluer ${ }^{191}$. Or, vu le déficit de redressement économique sur les quinze dernières années, le dispositif des pôles apparaît de moins en moins consensuel et de plus

189 J.-M. LAMBOTTE, «Les plans stratégiques des régions frontalières, source d'inspiration en cas de révision du SDER », Territoire(s) wallon(s), ${ }^{\circ}$ 6, 2011, p. 151-164.

190 CPDT, «Diagnostic territorial de la Wallonie », Namur, SPW, 2011, p. 239-241.

191 F. CARUSO et al., Évaluation du Plan d'actions prioritaires pour l'Avenir wallon. Rapport de synthèse. Annexes au rapport de synthèse, Période 2006-2008, Namur, IWEPS, 2009; Technopolis Group, Erdyn, IWEPS, Evvaluation du Plan Marshall 2.Vert. Évaluation thématique $n^{\circ} 1$ : Pôles de compétitivité, Rapport de recherche $\mathrm{n}^{\circ}$ 7, Namur, IWEPS, 2014 ; C. DUJARDIN, V. LOUIS, V. SCOURNEAU, La politique des pôles de compétitivité dans le cadre de la stratégie de spécialisation intelligente, Rapport de recherche n² 25, Namur, IWEPS, 2019. 
en plus polémique ${ }^{192}$. À son propos, il nous semble essentiel de chercher à objectiver le questionnement mis en exergue par Richard Shearmur ${ }^{193}$ sur les conditions de la concordance entre, d'une part, le ou les territoire(s) où une innovation voit le jour et, d'autre part, le ou les territoire(s) bénéficiaire(s) des nouvelles filières économiques induites.

En matière de politique publique, il semble également très important de questionner l'impact de la politique des zones franches (cf. supra), ainsi que l'impact des politiques d'investissements dans les infrastructures de transport et dans le foncier à vocation économique. En effet, des moyens considérables sont mobilisés pour ces politiques alors que leur impact sur la croissance n'est pas scientifiquement avéré. En outre, ces deux types de politiques contribuent à renforcer la périurbanisation et à dévitaliser les noyaux urbains. Cette situation ne manque pas de poser question vis-à-vis du rôle de la ressource territoriale des aménités urbaines sur les nouveaux lieux d'accueil des activités économiques. En parallèle, nous avons également vu dans l'état de l'art que, plutôt que les facteurs matériels du développement, ce sont principalement les facteurs immatériels - l'innovation, l'esprit d'entreprise, la formation, etc. - qui sont mis en exergue par la littérature scientifique. Sur un sujet proche, rappelons encore que l'argument selon lequel la Wallonie tire un avantage substantiel de sa situation géographique doit être relativisé (cf. supra, 3.1.1).

Afin de répondre à ces divers questionnements de manière suffisamment précise, il faudrait améliorer l'outillage statistique pour disposer de meilleures données. Par exemple, il est interpellant que nous ne disposions pas d'informations robustes sur les emplois de la base productive (cf. supra, 4.1.1). En outre, les bases de données sont lacunaires vis-à-vis de la question des investissements directs étrangers (IDE) ${ }^{194}$. Sans conteste, il aurait été très utile de traiter cette thématique dans la présente livraison du Courrier hebdomadaire. Malheureusement, l'absence de données suffisamment fines spatialement ne nous a pas permis de l'intégrer.

D’une manière plus générale, il importe de souligner que les travaux universitaires sur la question du redéploiement économique de la Wallonie sont au final assez peu nombreux. Ce constat, assez surprenant au regard de la hauteur des enjeux, trouve vraisemblablement son origine dans le fait que, pour le champ universitaire de l'économie, la thématique du développement régional est devenue moins porteuse pour les carrières académiques ${ }^{195}$. Cette situation interpelle car elle risque de limiter l'expertise sur laquelle les décisions de politique économique pourront s'appuyer. Dès lors, on ne peut qu'inviter la communauté universitaire à se pencher davantage sur cette question du développement régional, dans ses différentes échelles et dimensions. En parallèle, il s'agirait que les gouvernants régionaux soient davantage attentifs aux enseignements du monde académique et qu'ils érigent de nouveaux espaces et modalités de dialogue entre les sphères de la recherche, de l'administration et de l'entreprise.

192 J.-Y. Huwart, Pourquoi la Wallonie ne se redresse pas ?, op. cit.

193 R. SHEARMUR, «Innovation et développement territorial ? », op. cit., p. 17-27.

194 B. MÉRENNE-SCHOUMAKER, «La Belgique : une économie sous influence étrangère », op. cit. ; C. ALBESSART et al., Rapport sur l'économie wallonne, op. cit., p. 90.

195 M. BOURGEOIs et al., "Amélioration de l'attractivité et de la compétitivité du territoire wallon pour le secteur des services supérieurs », op. cit. 


\section{CENTRE DE RECHERCHE ET D'INFORMATION SOCIO-POLITIQUES}

Le CRISP, Centre de recherche et d'information socio-politiques, est un organisme indépendant. Ses travaux s'attachent à montrer les enjeux de la décision politique, à expliquer les mécanismes par lesquels elle s'opère, et à analyser le rôle des acteurs qui y prennent part, que ces acteurs soient politiques, économiques, sociaux, associatifs, etc.

Par ses publications, le CRISP met à la disposition d'un public désireux de comprendre la société belge des informations de haute qualité, dans un souci d'exactitude, de pertinence et de pluralisme. Son objectif est de livrer à ce public les clés d'explication du fonctionnement du système socio-politique belge et de mettre en évidence les structures réelles du pouvoir, en Belgique et dans le cadre de l'Union européenne.

Le Courrier hebdomadaire paraît au rythme de 40 numéros par an, certaines livraisons correspondant à deux numéros. Chaque livraison est une monographie consacrée à l'étude approfondie d'un aspect de la vie politique, économique ou sociale au sens large. La revue du CRISP constitue depuis 1959 une source d'information incontournable sur des sujets variés : partis politiques, organisations représentatives d'intérêts sociaux et groupes de pression divers, évolution et fonctionnement des institutions, négociations communautaires, histoire politique, groupes d'entreprises et structures du tissu économique, conflits sociaux, enseignement, immigration, vie associative et culturelle, questions environnementales, européennes, etc. C'est également dans le Courrier hebdomadaire que sont publiés les résultats des élections commentés par le CRISP.

Les auteurs publiés sont soit des chercheurs du CRISP, formés en diverses disciplines des sciences humaines, soit des spécialistes extérieurs provenant des mondes scientifique, associatif et socio-politique. Dans tous les cas, les textes sont revus avant publication par le rédacteur en chef et par un groupe d'experts sélectionnés en fonction de la problématique abordée, afin de garantir la fiabilité de l'information proposée. Cette fiabilité, ainsi que la rigoureuse objectivité du Courrier hebdomadaire, constituent les atouts principaux d'une revue dont la qualité est établie et reconnue depuis près de 60 ans.

Fondateur : Jules Gérard-Libois

Président : Vincent de Coorebyter

Équipe de recherche :

Benjamin Biard, Pierre Blaise (secrétaire général), Fabienne Collard, Jean Faniel (directeur général), Cédric Istasse, Vincent Lefebve, Caroline Sägesser, David Van Den Abbeel (coordinateur du secteur Économie), Marcus Wunderle

Conseil d'administration :

Louise-Marie Bataille, Jacques Brassinne de La Buissière (vice-président honoraire), Vincent de Coorebyter (président), Hugues Dumont, Éric Geerkens, Nadine Gouzée, Serge Govaert, Laura Iker, Patrick Lefevre, Michel Molitor (vice-président), Solveig Pahud, Pierre Reman, Robert Tollet (vice-président), Els Witte 


\section{Derniers numéros du Courrier hebdomadaire parus}

2440-2441 L'extrême droite en Europe centrale et orientale (2004-2019) Benjamin Biard

2438-2439 Les réformes de l'assurance chômage (2011-2019) Vincent Lefebve

2436-2437 Le statut pénal des parlementaires Marie Solbreux et Marc Verdussen

2435 Les évolutions électorales des partis politiques (1944-2019) III. Les familles politiques Cédric Istasse

2433-2434 Les résultats des élections fédérales et européennes du 26 mai 2019

Benjamin Biard, Pierre Blaise, Jean Faniel, Cédric Istasse et Caroline Sägesser

2431-2432 Les résultats des élections européennes de mai 2019 dans les États membres Camille Kelbel

2429-2430 Vers la loi du 28 mai 2002 relative à l'euthanasie (II). Une approche des débats parlementaires et de leurs prolongements Marie-Luce Delfosse

2427-2428 Vers la loi du 28 mai 2002 relative à l'euthanasie (I). Une approche des débats parlementaires Marie-Luce Delfosse

2426 Le dialogue citoyen permanent en Communauté germanophone Christoph Niessen et Min Reuchamps

2424-2425 Grèves et conflictualité sociale en 2018 II. Conflits d'entreprise Iannis Gracos

2422-2423 Grèves et conflictualité sociale en 2018 I. Mobilisations transversales Iannis Gracos

2420-2421 L'extrême droite en Europe occidentale (2004-2019) Benjamin Biard

La collection intégrale du Courrier hebdomadaire est accessible sur www.cairn.info. L'accès est gratuit pour les numéros parus avant 2018.

Découvrez notre catalogue complet incluant nos autres publications sur www.crisp.be.

Pour être informé de nos publications dès leur parution, inscrivez-vous en ligne à notre lettre d'information électronique. 